Supporting Information

\title{
Synthesis of Sea Cucumber Saponins with Antitumor Activities
}

Xiaofei Shao, ${ }^{\dagger}$ Xiaobo Wang, ${ }^{\ddagger}$ Kaidi Zhu, ${ }^{\dagger}$ Yongjun Dang,,${ }^{\ddagger}$ and Biao Yu ${ }^{*},, \S$

†State Key Laboratory of Bioorganic and Natural Products Chemistry, Center for

Excellence in Molecular Synthesis, Shanghai Institute of Organic Chemistry, University of Chinese Academy of Sciences, Chinese Academy of Sciences, 345 Lingling Road, Shanghai 200032, China

*Key Laboratory of Metabolism and Molecular Medicine, the Ministry of Education, Department of Biochemistry and Molecular Biology, School of Basic Medical Sciences, Fudan University, Shanghai 200032, China

${ }^{\S}$ School of Chemistry and Materials Science, Hangzhou Institute for Advanced Study, University of Chinese Academy of Sciences, 1 Sub-lane Xiangshan, Hangzhou 310024, China

Comparison of the ${ }^{13} \mathrm{C}$ NMR data of the synthetic samples with those reported for the natural products ................................................................................................................S2

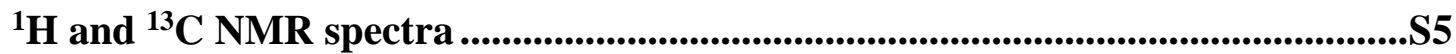

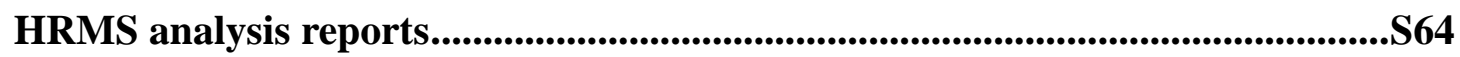


Comparison of the ${ }^{13} \mathrm{C}$ NMR data of the synthetic samples with those reported for the natural products

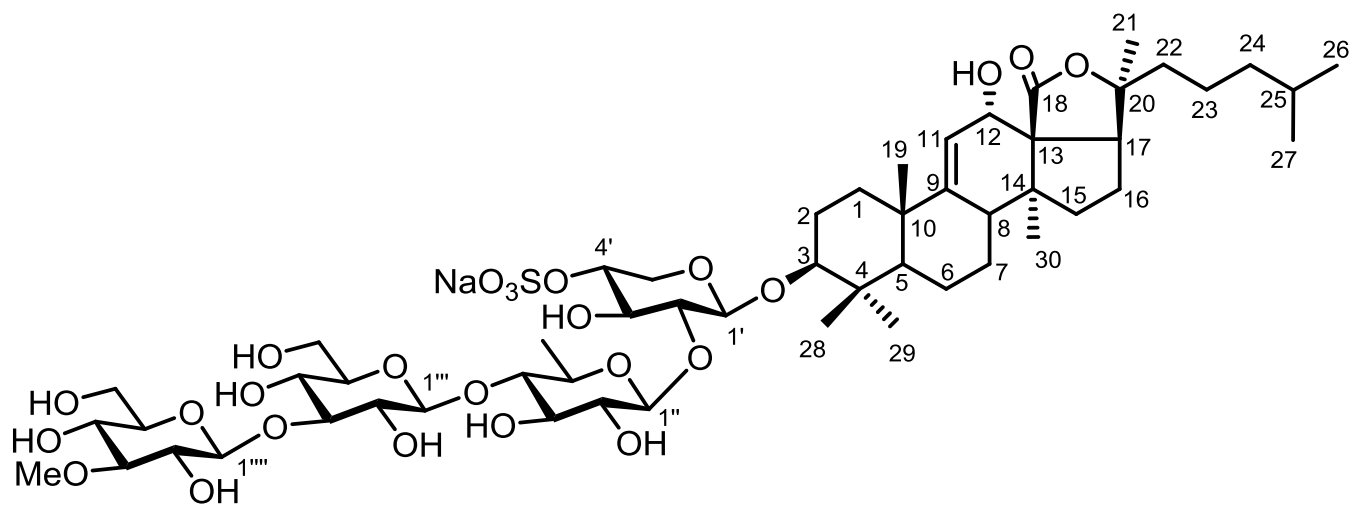

Table S1. Comparison of the ${ }^{13} \mathrm{C}$ NMR data of the synthetic 3 with those reported for the natural pervicoside $\mathrm{C}$.

\begin{tabular}{|c|c|c|c|c|c|}
\hline $\mathrm{C}$ & Reported & Synthetic & $\mathrm{C}$ & Reported & Synthetic \\
\hline 1 & 36.5 & 36.4 & Xyl & & \\
\hline 2 & 27.0 & 27.0 & $1^{\prime}$ & 105.1 & 105.2 \\
\hline 3 & 88.8 & 88.8 & $2^{\prime}$ & 83.3 & 83.4 \\
\hline 4 & 40.0 & 40.0 & $3^{\prime}$ & 75.2 & 75.2 \\
\hline 5 & 52.9 & 52.8 & $4^{\prime}$ & 76.2 & 76.3 \\
\hline 6 & 21.3 & 21.2 & $5^{\prime}$ & 64.2 & 64.3 \\
\hline 7 & 28.8 & 28.8 & Qui & & \\
\hline 8 & 40.2 & 40.1 & $1 "$ & 105.3 & 105.5 \\
\hline 9 & 153.1 & 153.0 & $2^{\prime \prime}$ & 75.4 & 75.4 \\
\hline 10 & 39.6 & 39.6 & 3" & 75.8 & 75.7 \\
\hline 11 & 116.2 & 116.2 & 4" & 86.6 & 86.5 \\
\hline 12 & 68.3 & 68.2 & 5" & 71.9 & 72.0 \\
\hline 13 & 64.2 & 64.1 & 6" & 18.0 & 18.0 \\
\hline 14 & 46.6 & 46.6 & Glu & & \\
\hline 15 & 37.2 & 37.2 & $1^{\prime \prime \prime}$ & 104.7 & 104.8 \\
\hline 16 & 24.2 & 24.1 & $2^{\prime \prime \prime}$ & 73.9 & 74.0 \\
\hline 17 & 47.1 & 47.0 & 3'" & 88.0 & 88.0 \\
\hline 18 & 177.2 & 177.3 & 4"' & 69.5 & 69.3 \\
\hline
\end{tabular}




\begin{tabular}{|l|l|l|l|l|l|}
\hline 19 & 22.5 & 22.5 & $5 ' \prime$ & 77.6 & 77.6 \\
\hline 20 & 84.7 & 84.8 & $6^{\prime \prime \prime}$ & 61.8 & 61.6 \\
\hline 21 & 26.4 & 26.4 & MeGlu & & \\
\hline 22 & 39.6 & 39.6 & $1^{\prime \prime \prime}$ & 105.8 & 105.9 \\
\hline 23 & 22.2 & 22.2 & $2^{\prime \prime \prime \prime}$ & 74.8 & 74.9 \\
\hline 24 & 39.5 & 39.4 & $3^{\prime \prime \prime}$ & 87.9 & 88.0 \\
\hline 25 & 28.1 & 28.0 & $4^{\prime \prime \prime \prime}$ & 70.6 & 70.5 \\
\hline 26 & 22.6 & 22.6 & $5^{\prime \prime \prime}$ & 78.2 & 78.2 \\
\hline 27 & 22.6 & 22.6 & $66^{\prime \prime \prime}$ & 62.1 & 62.0 \\
\hline 28 & 16.7 & 16.6 & OMe & 60.7 & 60.8 \\
\hline 29 & 27.9 & 28.0 & & & \\
\hline 30 & 22.0 & 22.0 & & & \\
\hline
\end{tabular}

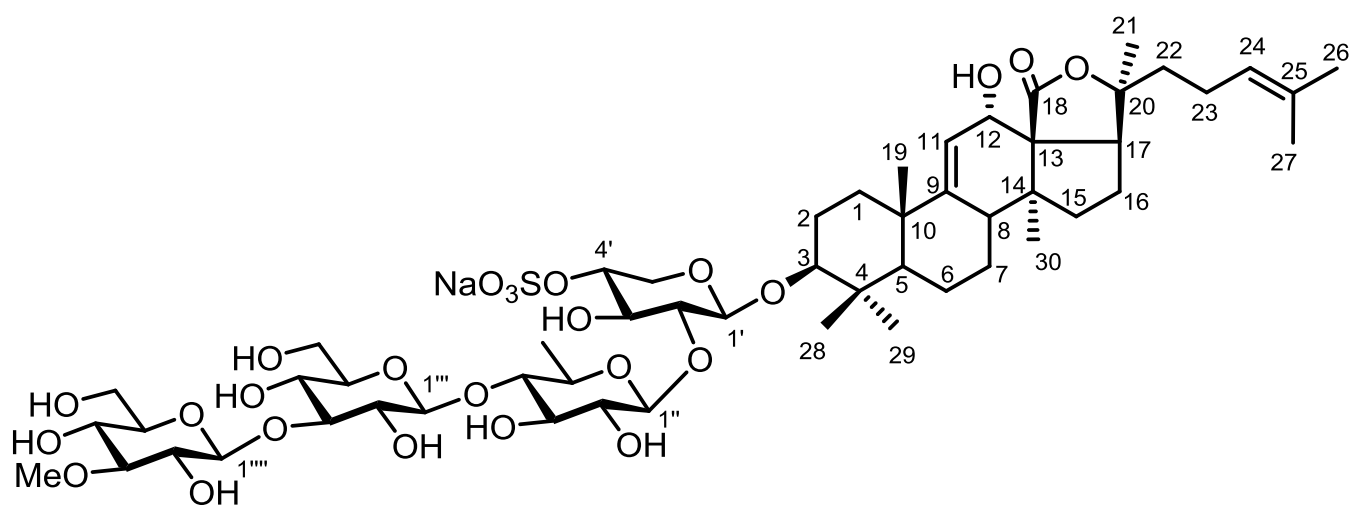

Table S2. Comparison of the ${ }^{13} \mathrm{C}$ NMR data for aglycone of the synthetic 2 with those reported for Ds-pervicoside $\mathrm{B}$.

\begin{tabular}{|c|c|c|}
\hline C & Reported & Synthetic \\
\hline 1 & 36.8 & 36.7 \\
\hline 2 & 27.3 & 27.3 \\
\hline 3 & 89.1 & 89.1 \\
\hline 4 & 40.2 & 40.2 \\
\hline 5 & 53.2 & 53.0 \\
\hline 6 & 21.4 & 21.5 \\
\hline 7 & 29.0 & 29.1 \\
\hline
\end{tabular}




\begin{tabular}{|c|c|c|}
\hline 8 & 40.4 & 40.4 \\
\hline 9 & 153.6 & 153.3 \\
\hline 10 & 39.8 & 39.8 \\
\hline 11 & 116.4 & 116.4 \\
\hline 12 & 68.5 & 68.4 \\
\hline 13 & 64.4 & 64.4 \\
\hline 14 & 46.8 & 46.9 \\
\hline 15 & 37.3 & 37.4 \\
\hline 16 & 24.3 & 24.5 \\
\hline 17 & 47.2 & 47.2 \\
\hline 18 & 177.5 & 177.5 \\
\hline 19 & $18.3^{\mathrm{a}}$ & 22.7 \\
\hline 20 & 84.7 & 84.8 \\
\hline 21 & 26.4 & 26.5 \\
\hline 22 & 39.6 & 39.7 \\
\hline 23 & 23.4 & 23.6 \\
\hline 24 & 124.5 & 124.5 \\
\hline 25 & 132.1 & 132.0 \\
\hline 26 & 25.7 & 25.9 \\
\hline 27 & 17.7 & 17.9 \\
\hline 28 & 16.8 & 16.9 \\
\hline 29 & 28.3 & 28.3 \\
\hline 30 & 22.1 & 22.3 \\
\hline
\end{tabular}

${ }^{\text {a }}$ The signal of $18.3 \mathrm{ppm}$ is most likely assigned incorrectly for $\mathrm{C} 19$, whereas the signal of $\mathrm{C} 19$ in pervicoside $\mathrm{C}$ is reported at $22.5 \mathrm{ppm}$. 


\section{${ }^{1} \mathrm{H}$ and ${ }^{13} \mathrm{C}$ NMR spectra}

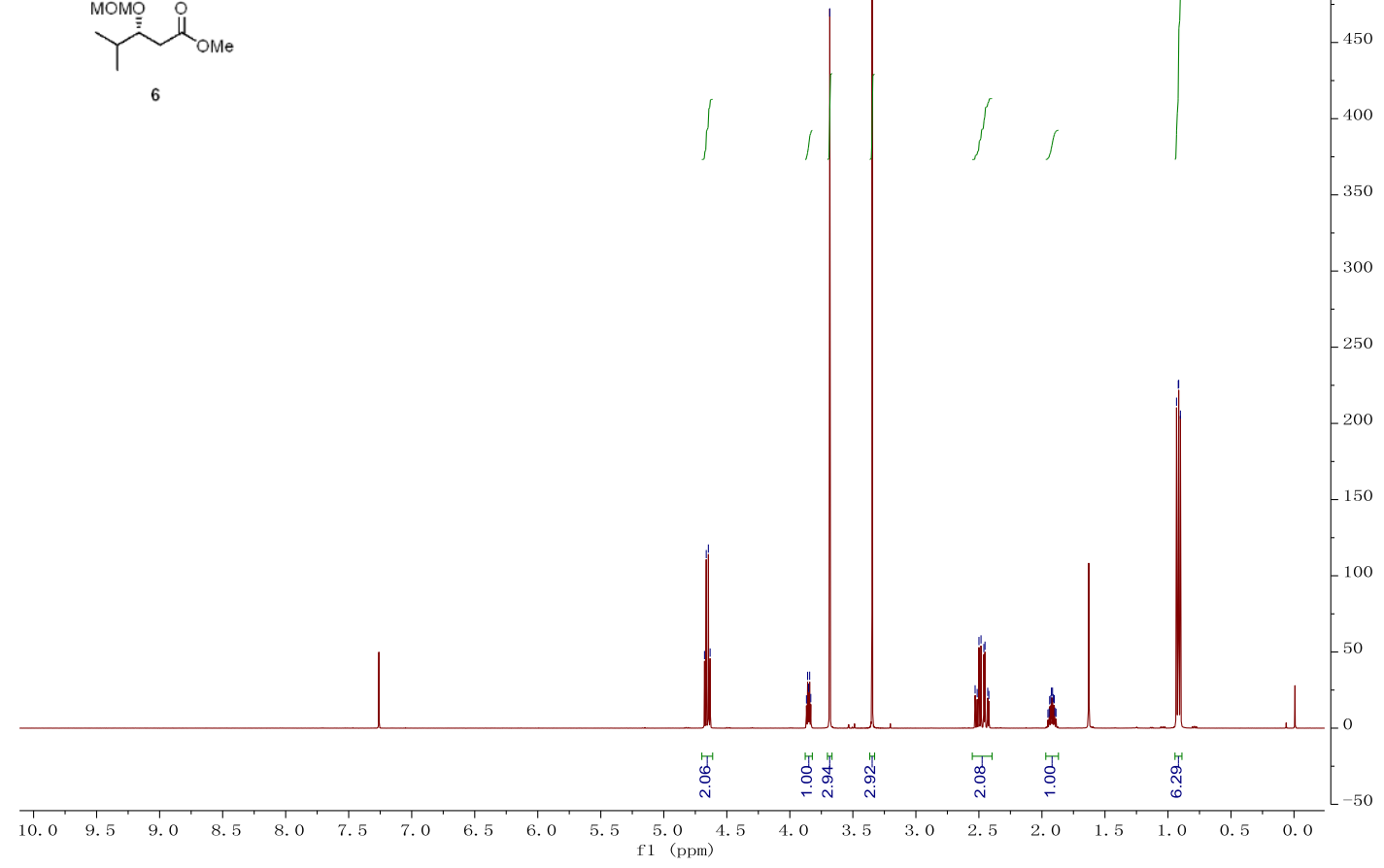

Figure S1. ${ }^{1} \mathrm{H}$ NMR spectrum of compound $6\left(500 \mathrm{MHz}, \mathrm{CDCl}_{3}\right)$

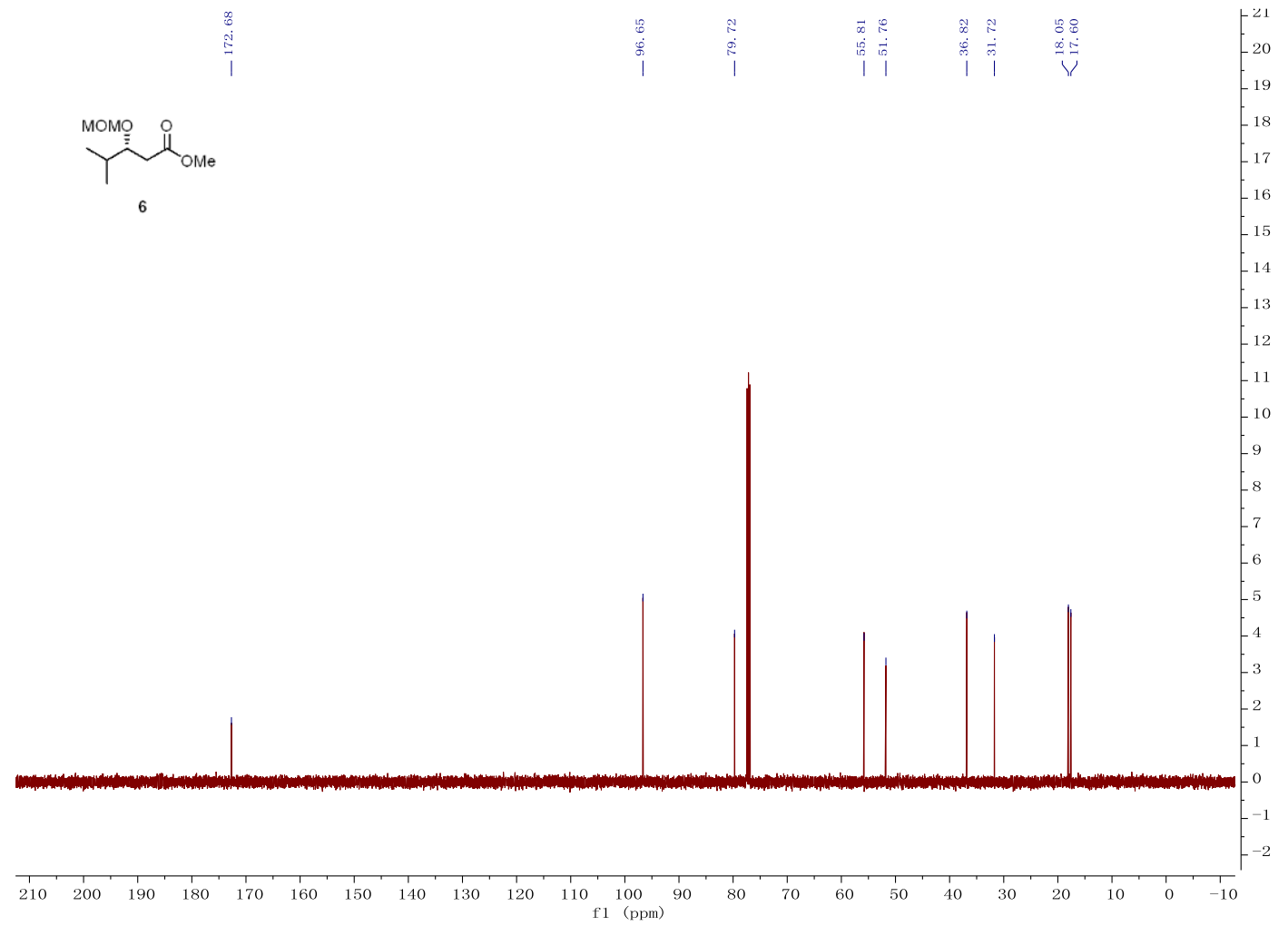

Figure S2. ${ }^{13} \mathrm{C}$ NMR spectrum of compound $6\left(125 \mathrm{MHz}, \mathrm{CDCl}_{3}\right)$ 


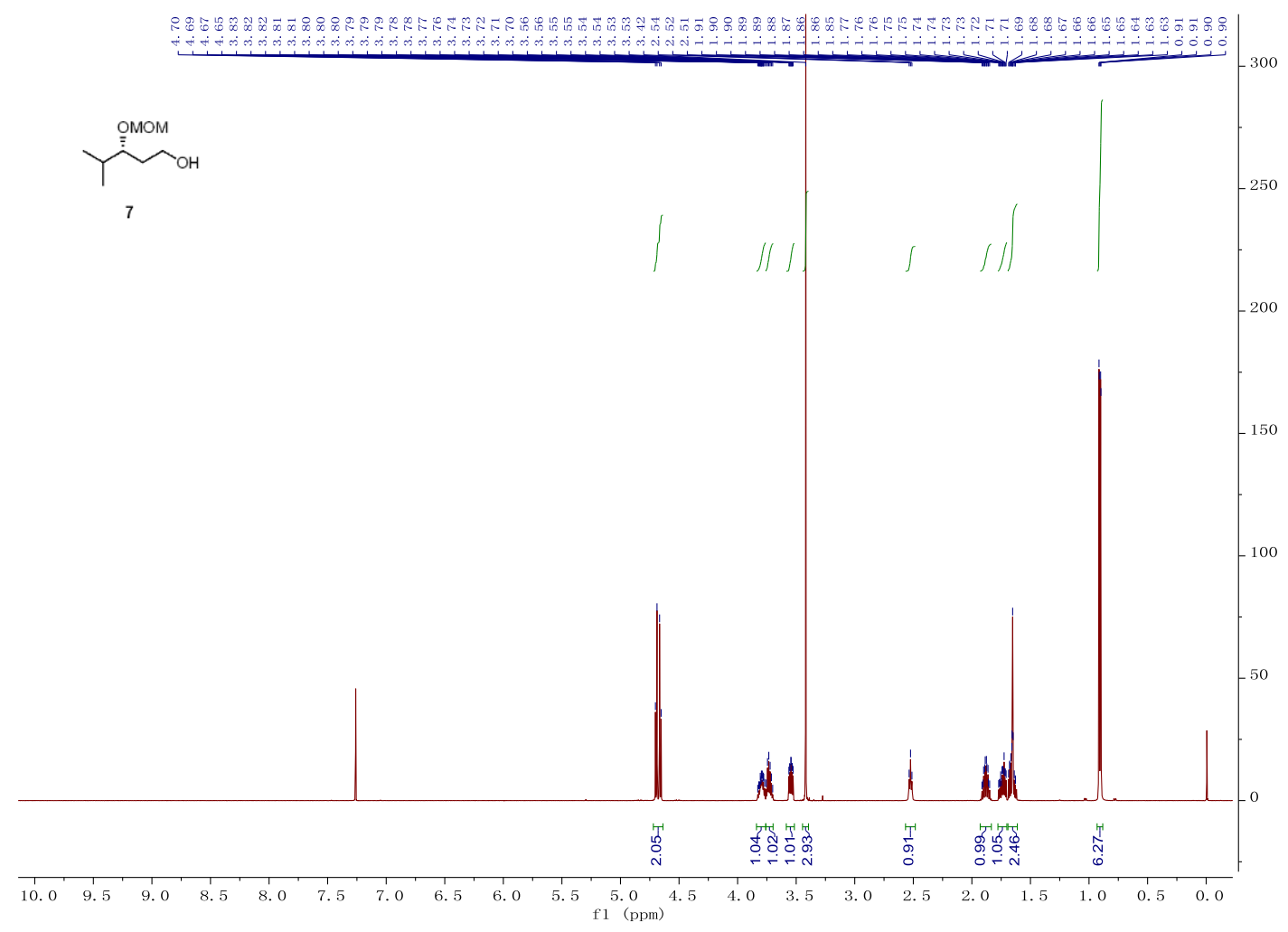

Figure S3. ${ }^{1} \mathrm{H}$ NMR spectrum of compound $7\left(500 \mathrm{MHz}, \mathrm{CDCl}_{3}\right)$

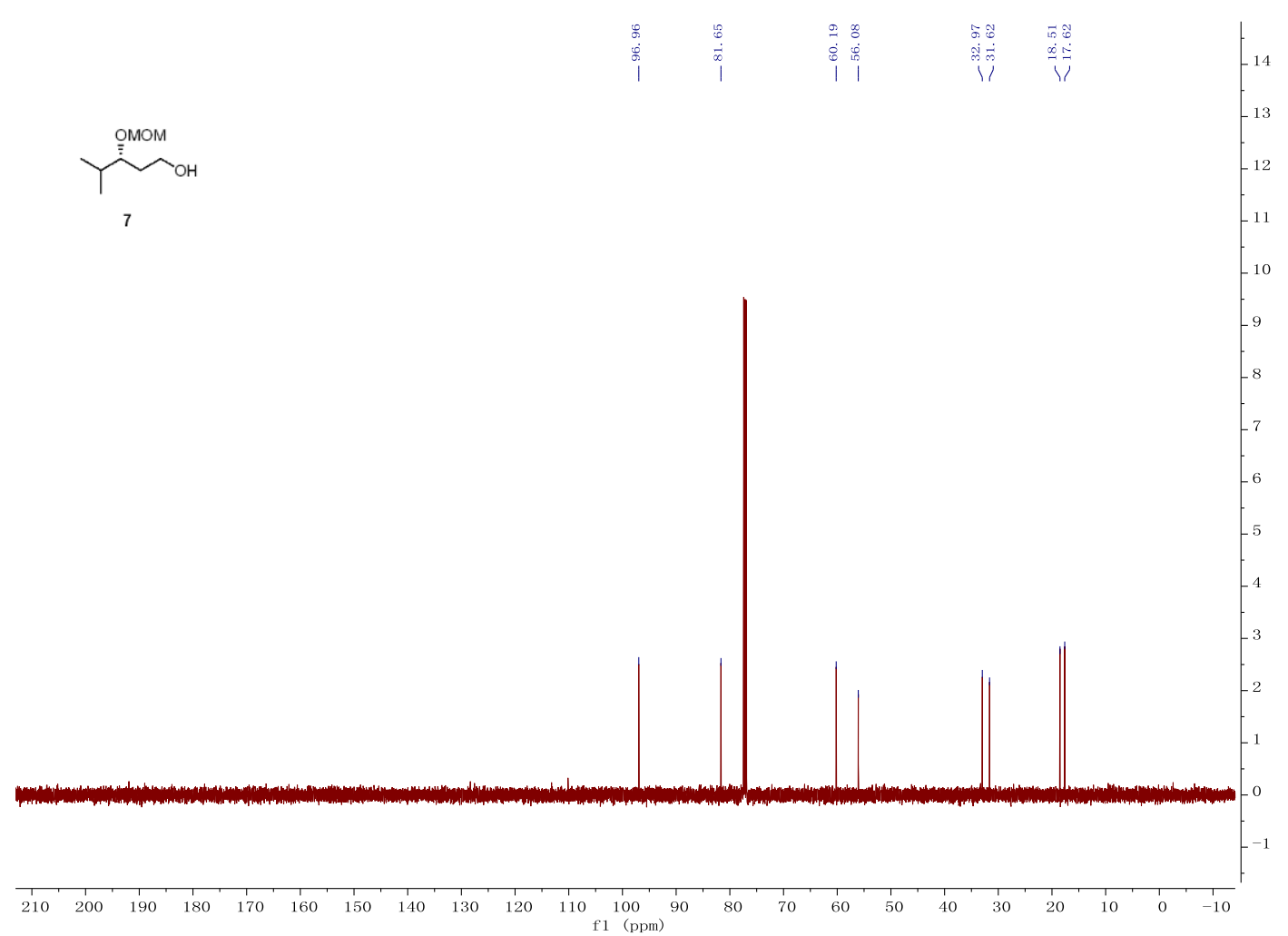

Figure S4. ${ }^{13} \mathrm{C}$ NMR spectrum of compound $7\left(125 \mathrm{MHz}, \mathrm{CDCl}_{3}\right)$ 


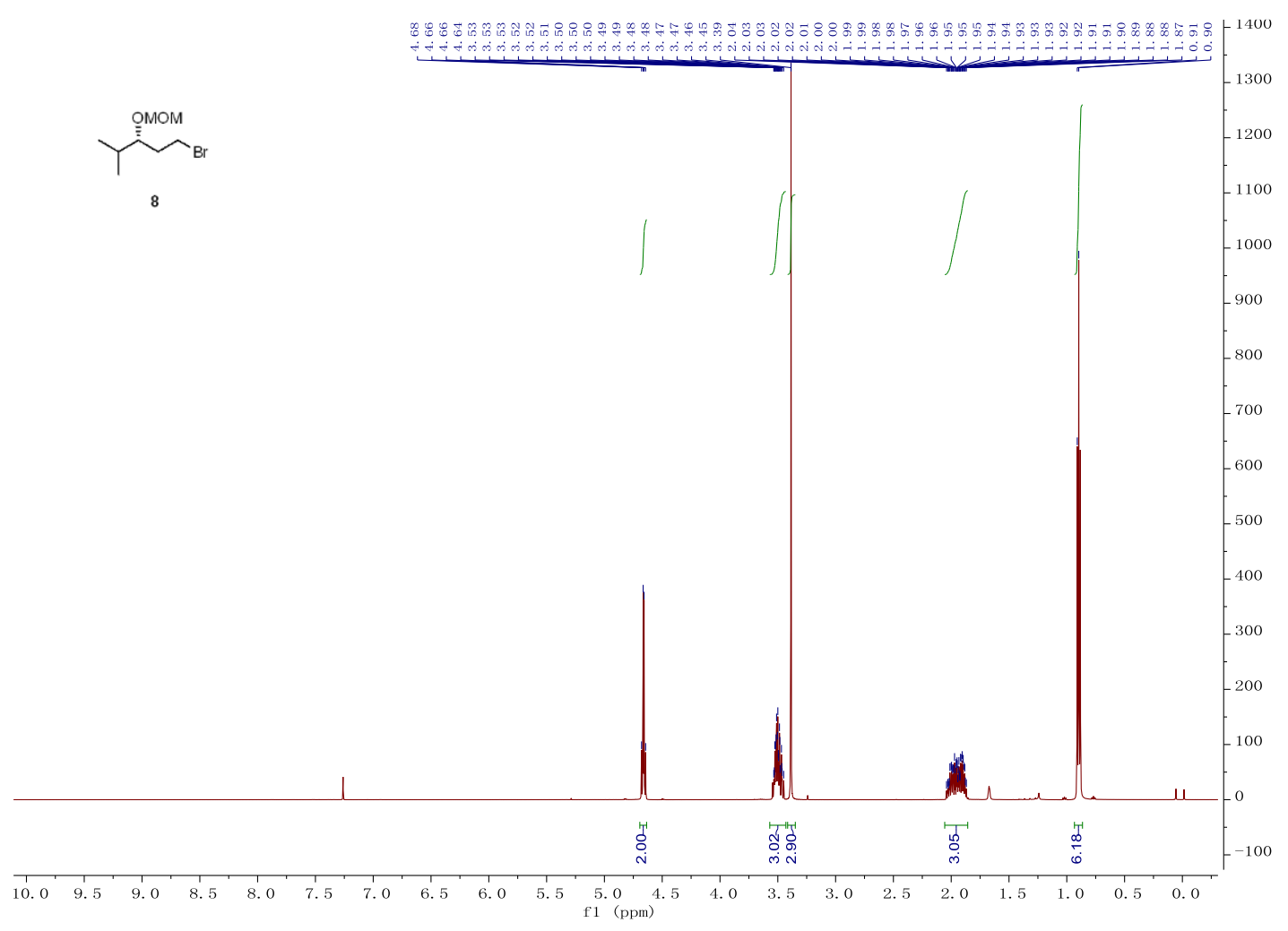

Figure S5. ${ }^{1} \mathrm{H}$ NMR spectrum of compound $8\left(500 \mathrm{MHz}, \mathrm{CDCl}_{3}\right)$

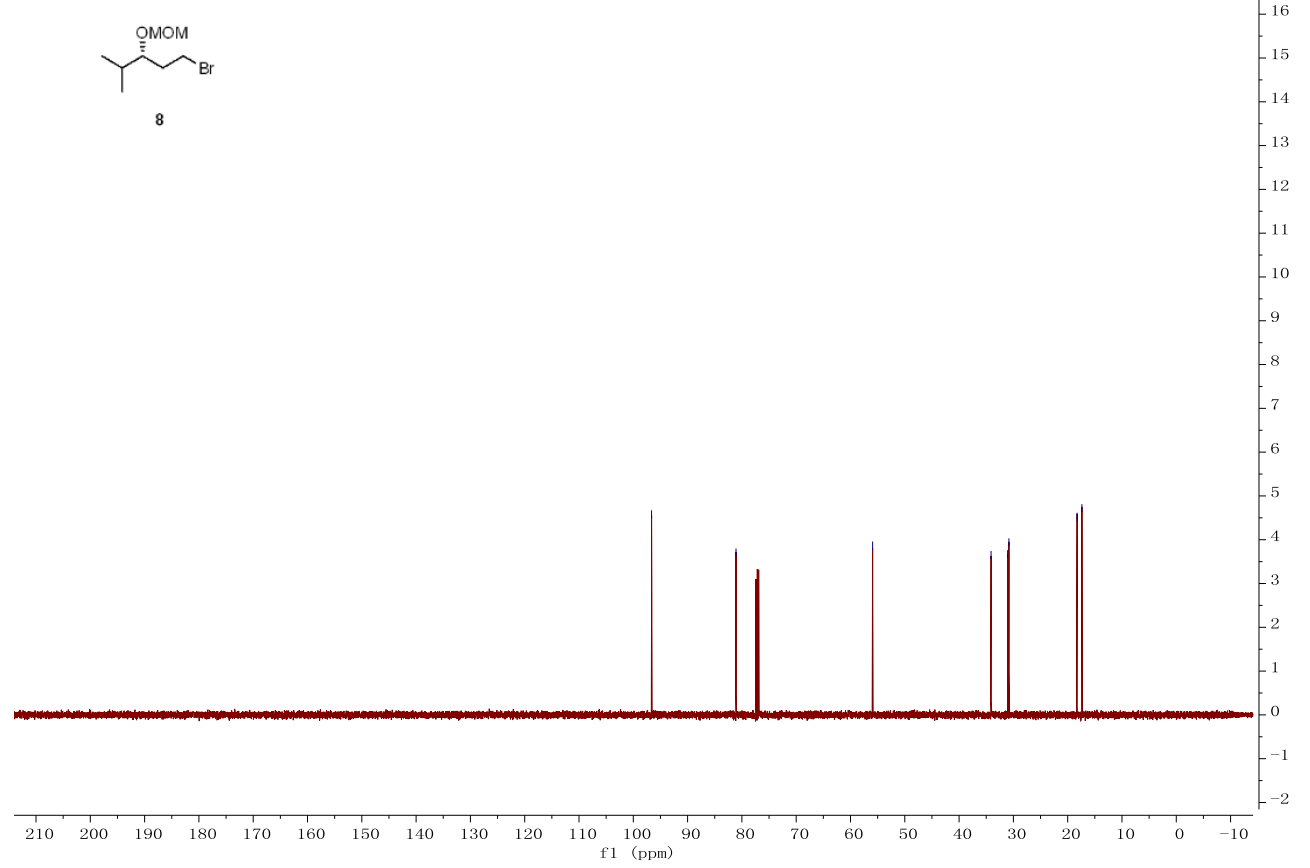

Figure S6. ${ }^{13} \mathrm{C}$ NMR spectrum of compound $8\left(125 \mathrm{MHz}, \mathrm{CDCl}_{3}\right)$ 


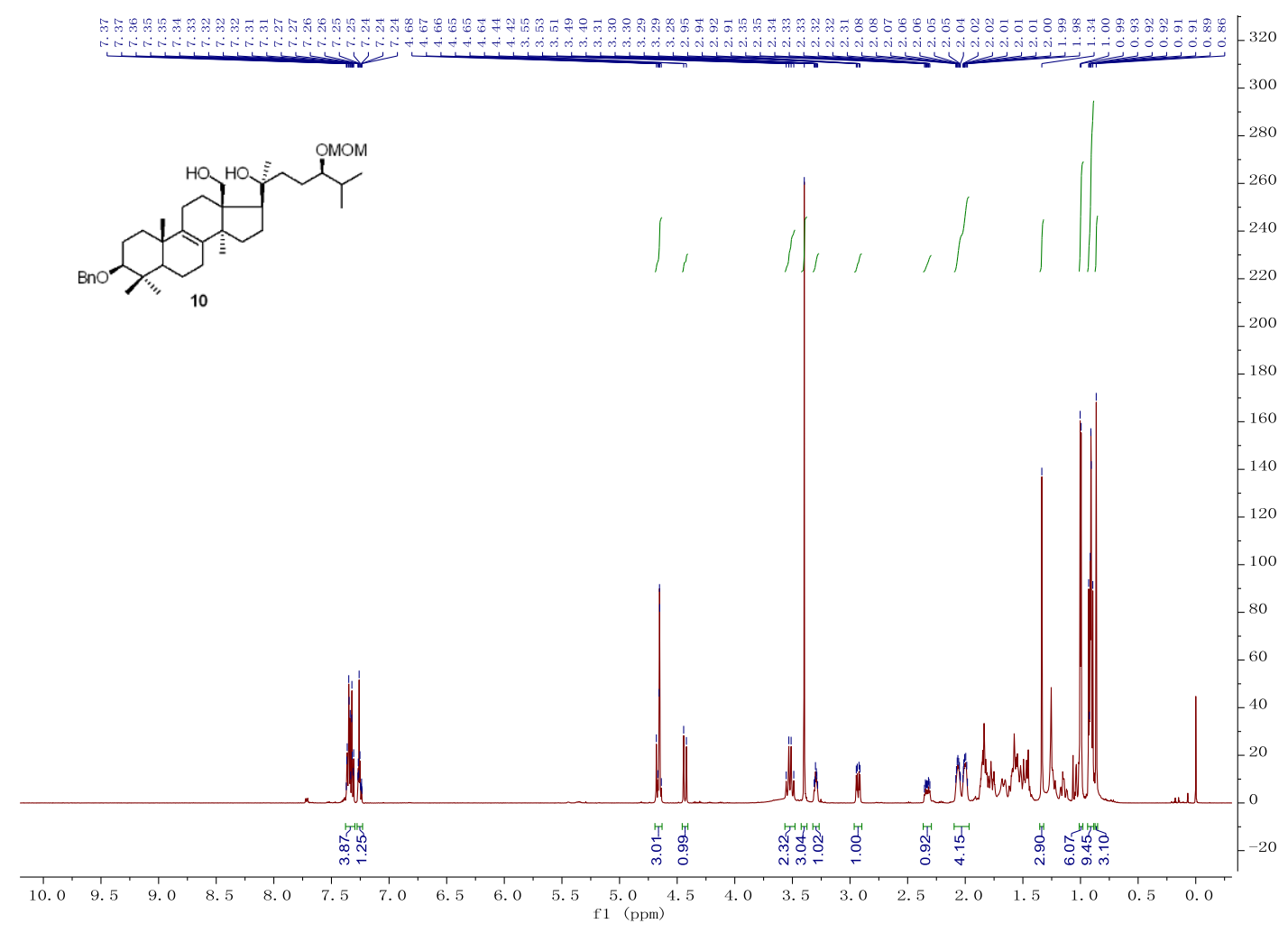

Figure S7. ${ }^{1} \mathrm{H}$ NMR spectrum of compound $10\left(500 \mathrm{MHz}, \mathrm{CDCl}_{3}\right)$

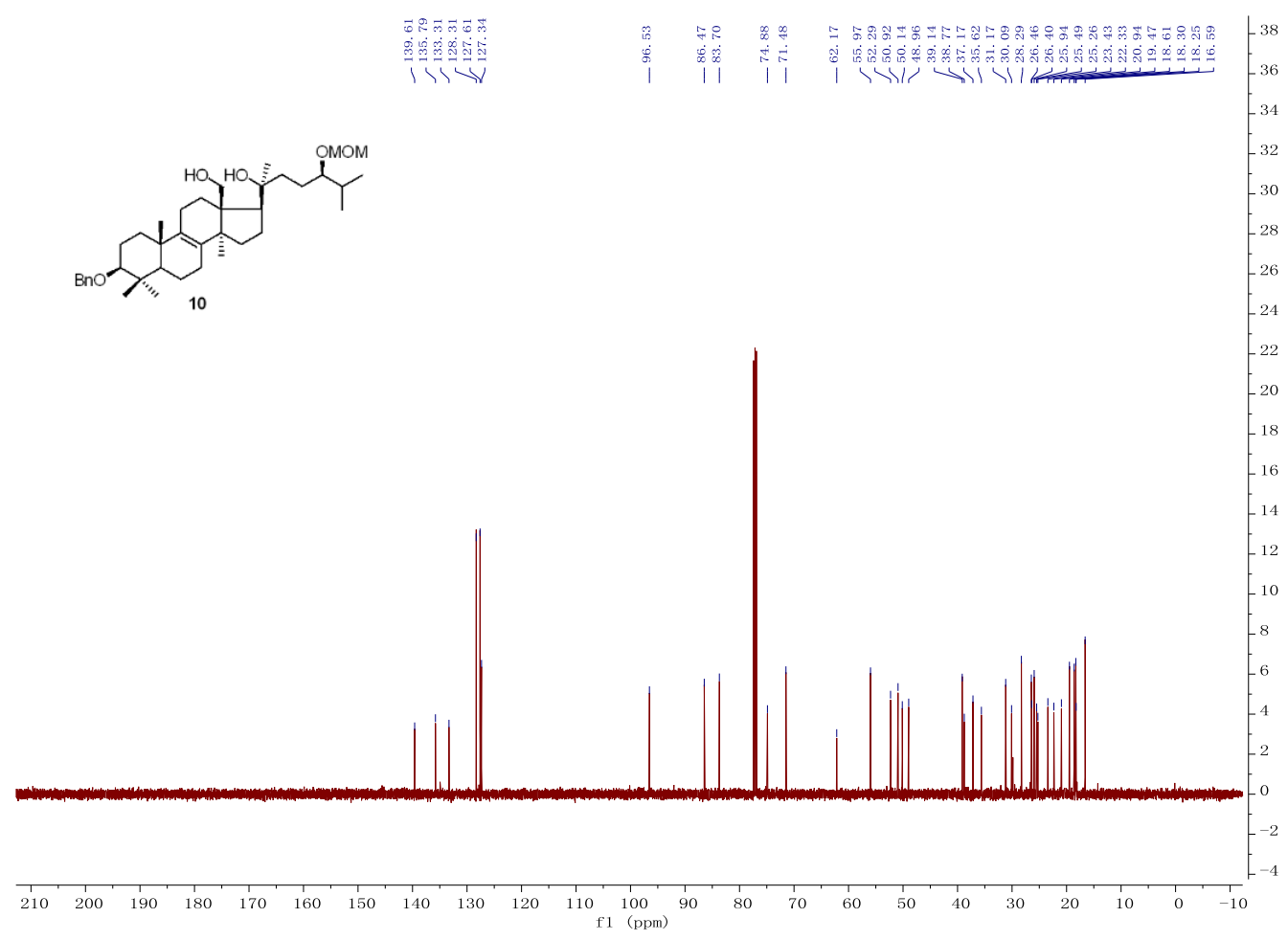

Figure S8. ${ }^{13} \mathrm{C}$ NMR spectrum of compound $\mathbf{1 0}\left(125 \mathrm{MHz}, \mathrm{CDCl}_{3}\right)$ 


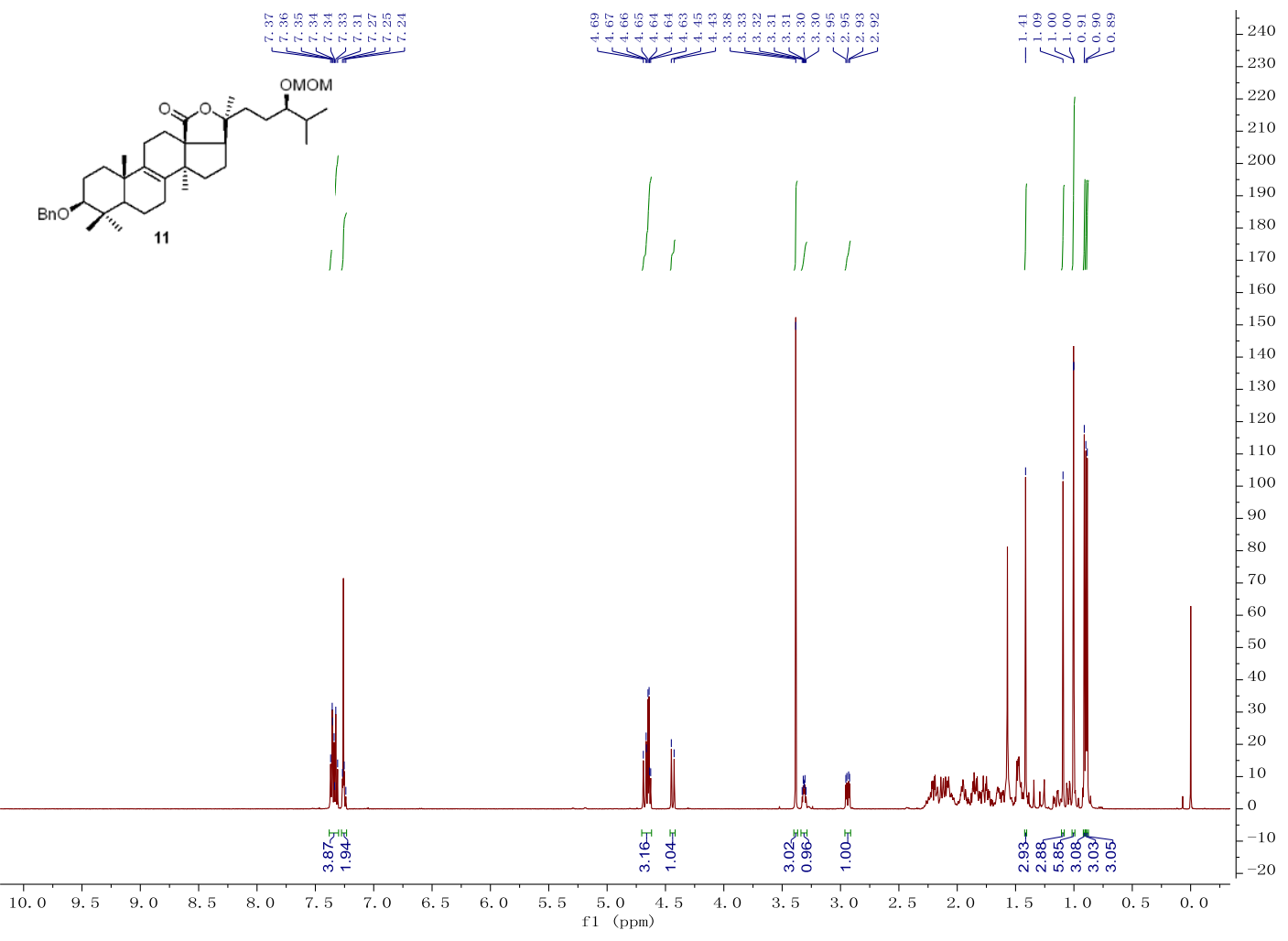

Figure S9. ${ }^{1} \mathrm{H}$ NMR spectrum of compound $11\left(500 \mathrm{MHz}, \mathrm{CDCl}_{3}\right)$
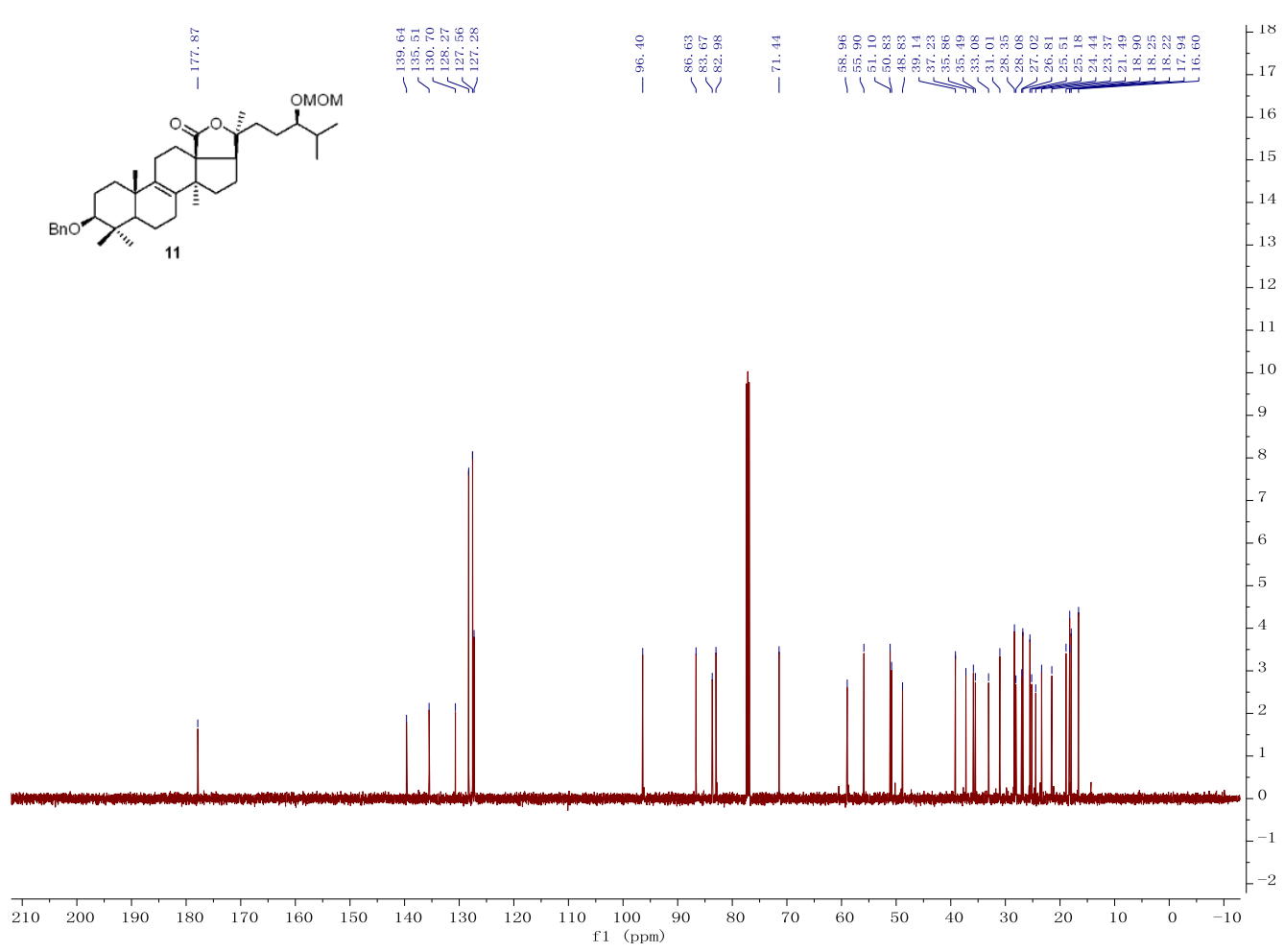

Figure S10. ${ }^{13} \mathrm{C}$ NMR spectrum of compound $11\left(125 \mathrm{MHz}, \mathrm{CDCl}_{3}\right)$ 


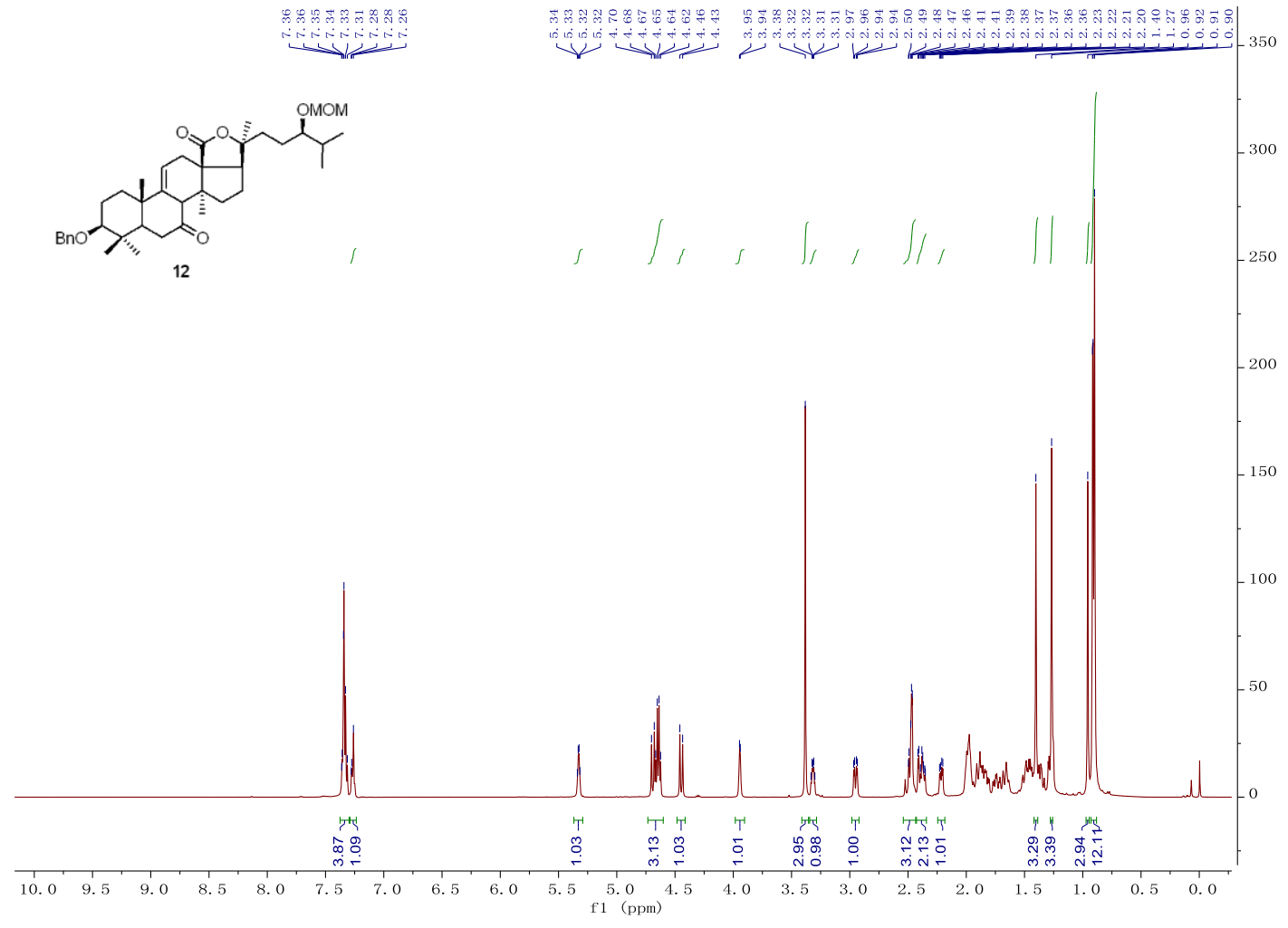

Figure S11. ${ }^{1} \mathrm{H}$ NMR spectrum of compound $12\left(500 \mathrm{MHz}, \mathrm{CDCl}_{3}\right)$

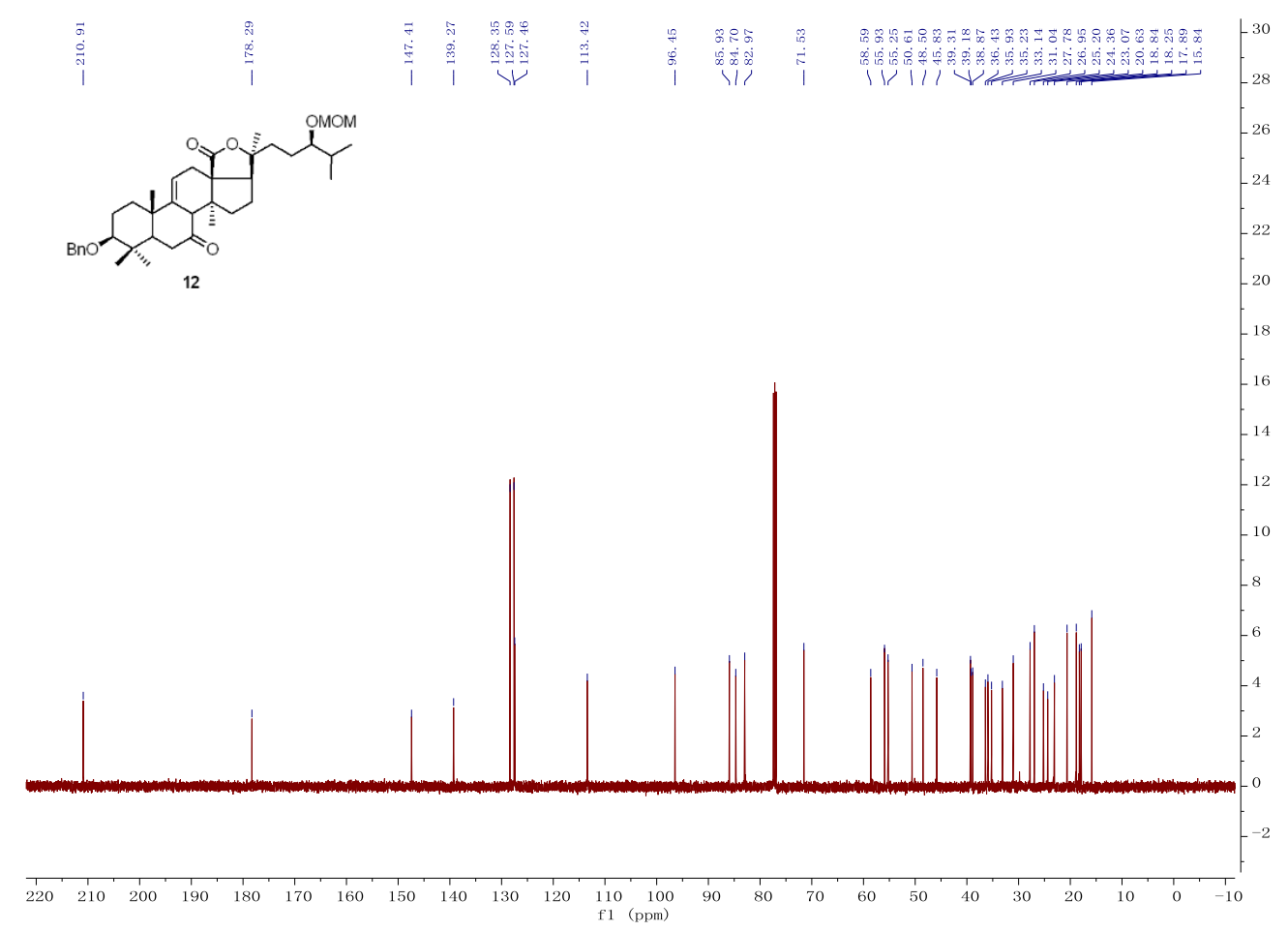

Figure S12. ${ }^{13} \mathrm{C}$ NMR spectrum of compound $12\left(125 \mathrm{MHz}, \mathrm{CDCl}_{3}\right)$ 


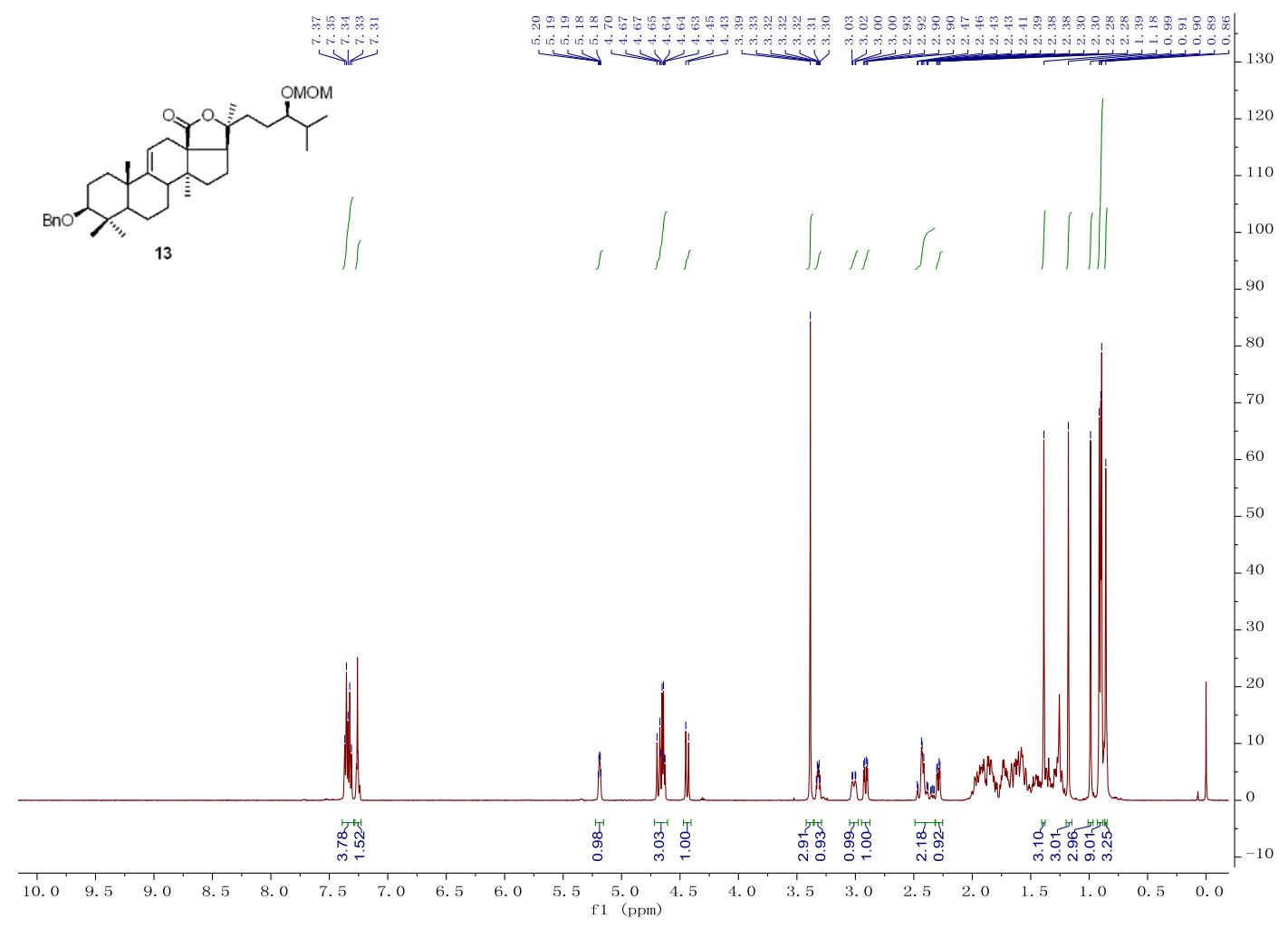

Figure S13. ${ }^{1} \mathrm{H}$ NMR spectrum of compound $13\left(500 \mathrm{MHz}, \mathrm{CDCl}_{3}\right)$

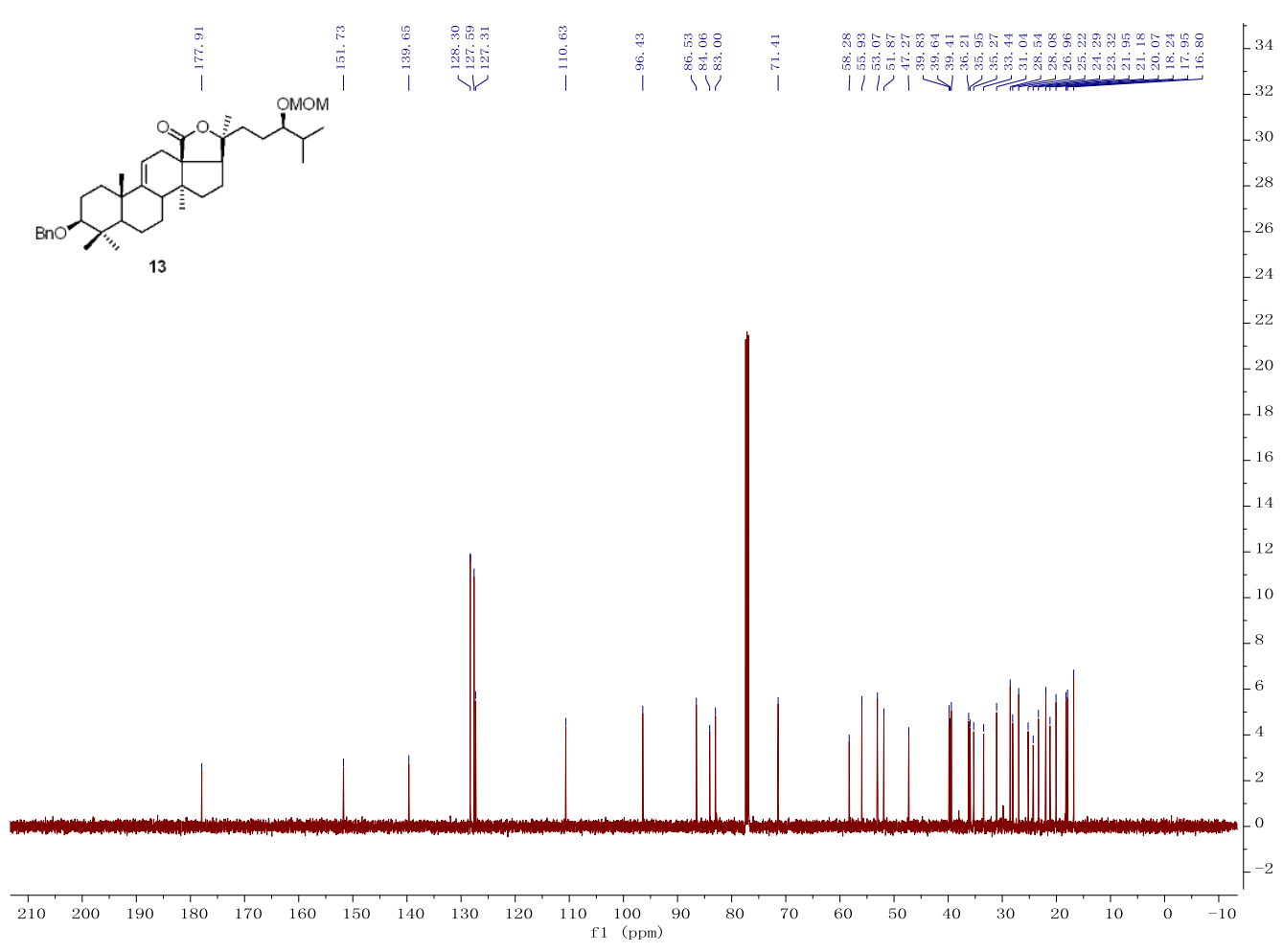

Figure S14. ${ }^{13} \mathrm{C}$ NMR spectrum of compound $13\left(125 \mathrm{MHz}, \mathrm{CDCl}_{3}\right)$ 


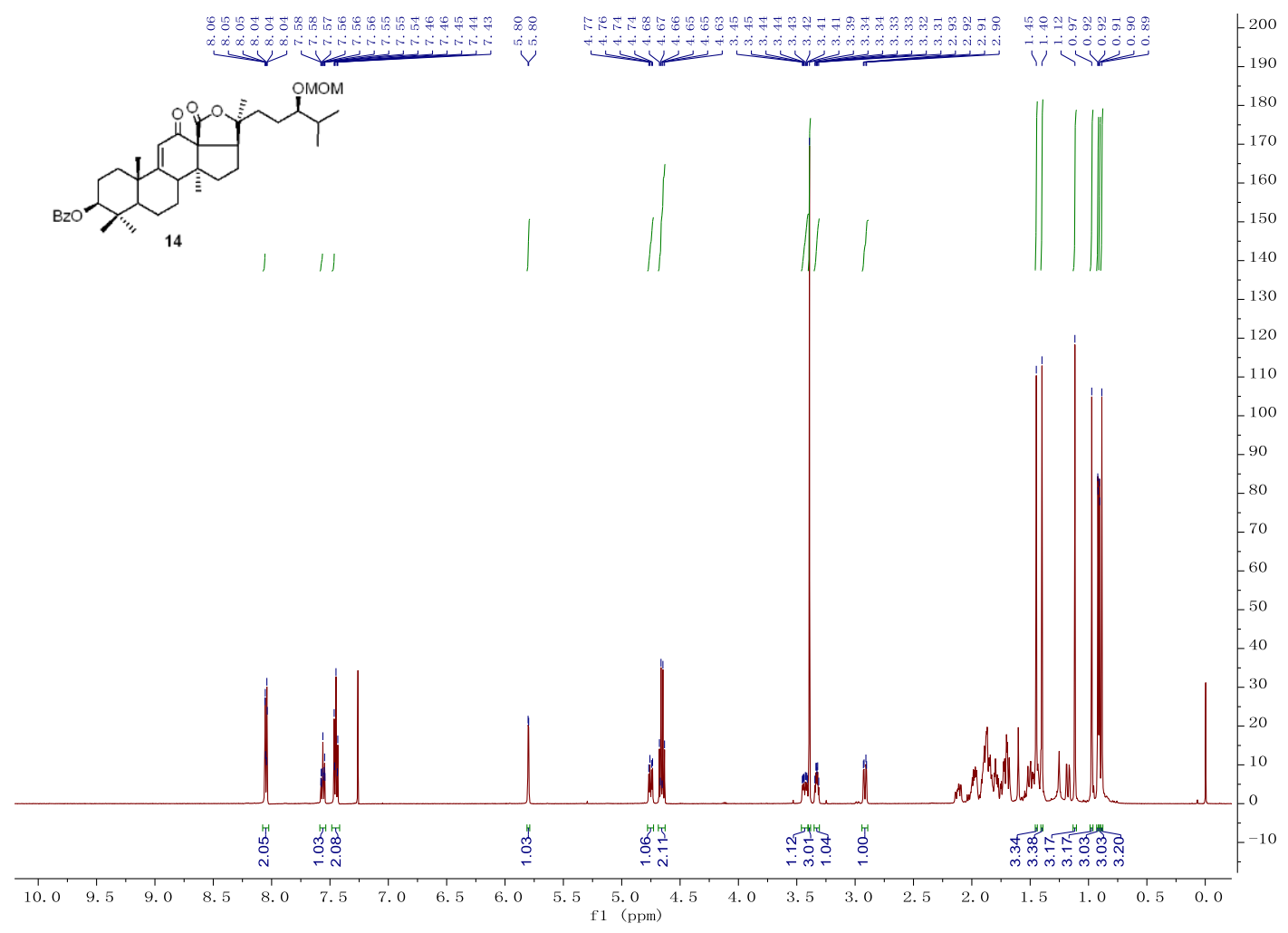

Figure S15. ${ }^{1} \mathrm{H}$ NMR spectrum of compound $14\left(500 \mathrm{MHz}, \mathrm{CDCl}_{3}\right)$

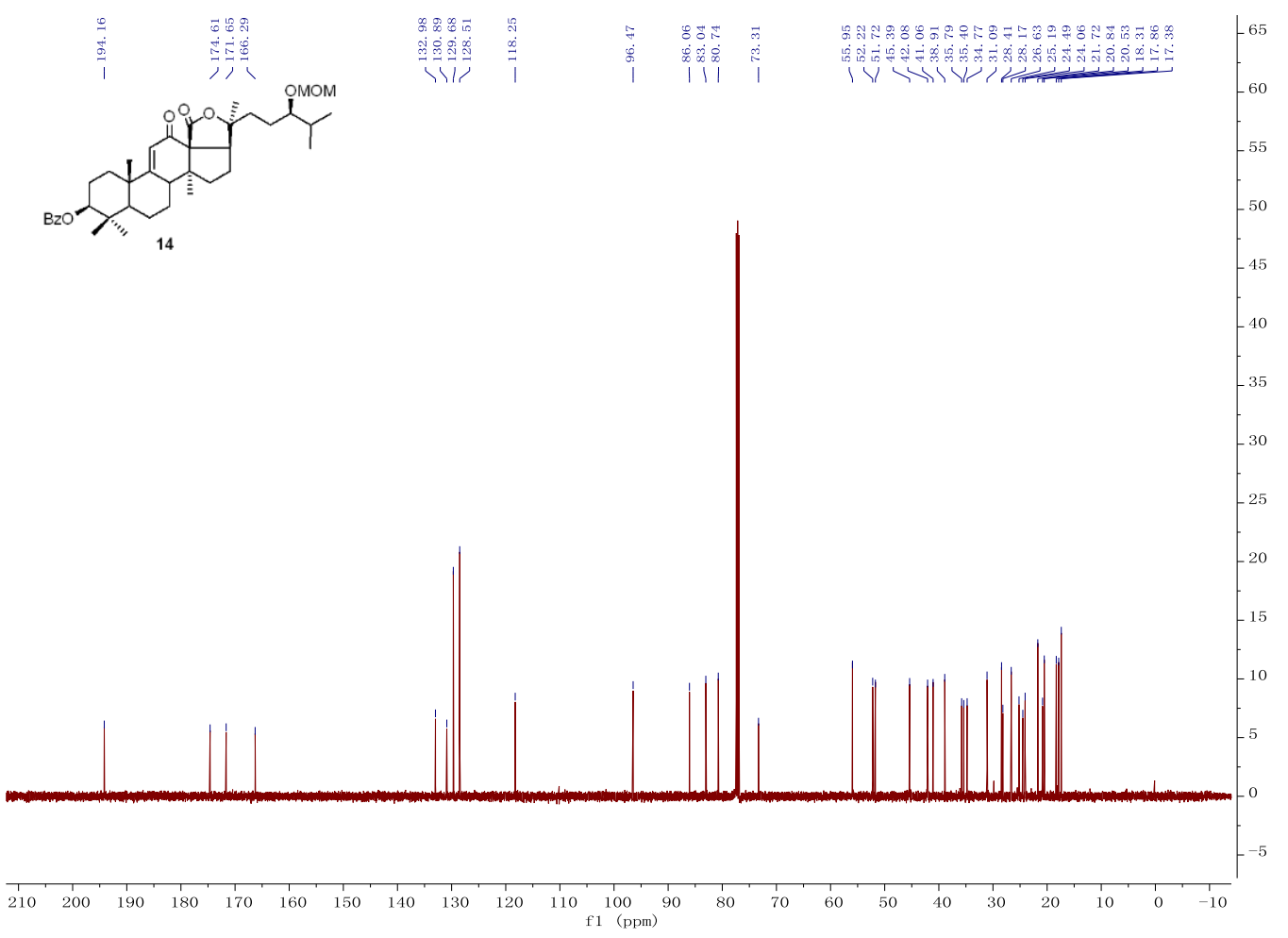

Figure S16. ${ }^{13} \mathrm{C}$ NMR spectrum of compound $14\left(125 \mathrm{MHz}, \mathrm{CDCl}_{3}\right)$ 


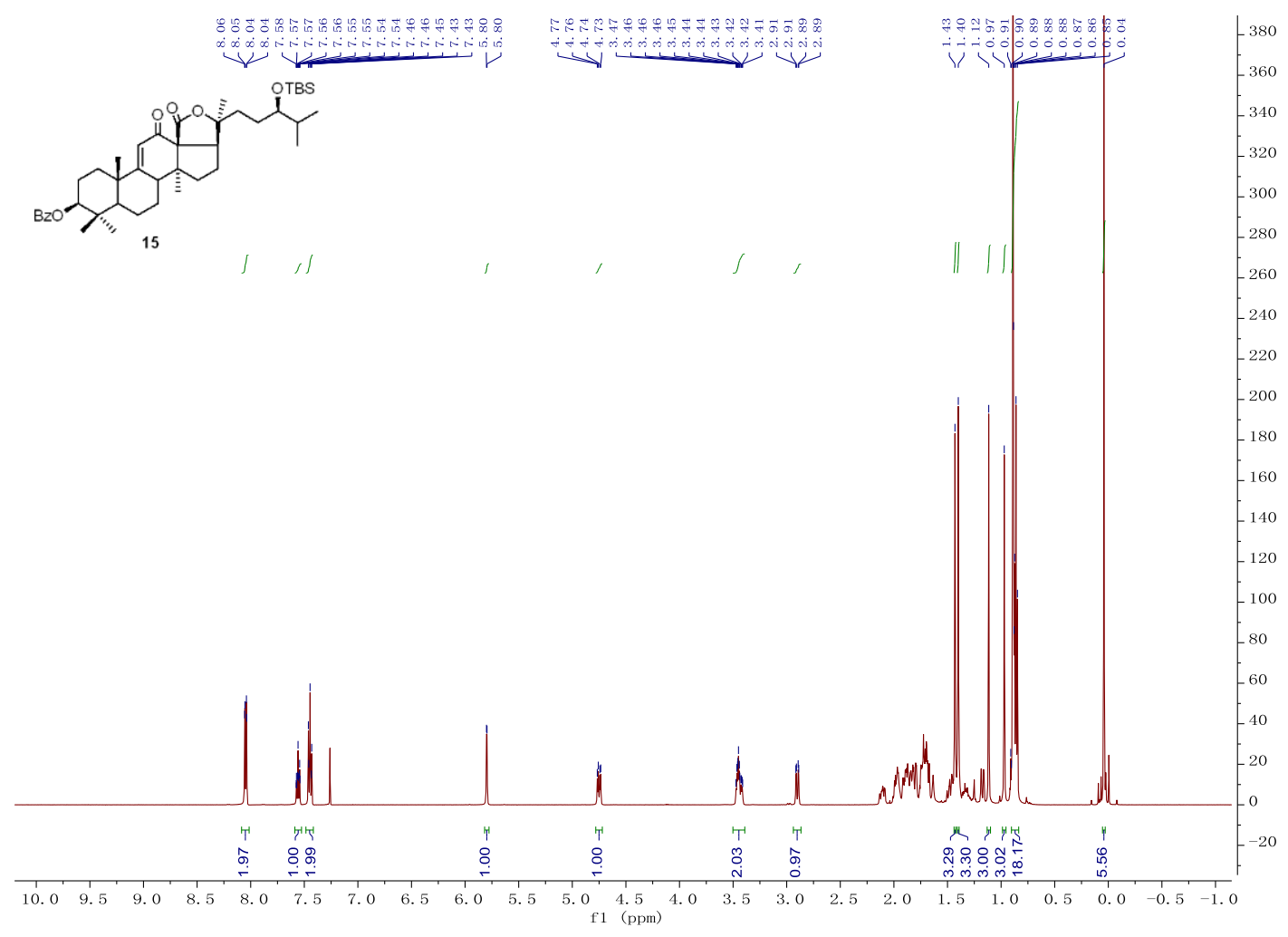

Figure S17. ${ }^{1} \mathrm{H}$ NMR spectrum of compound $15\left(500 \mathrm{MHz}, \mathrm{CDCl}_{3}\right)$
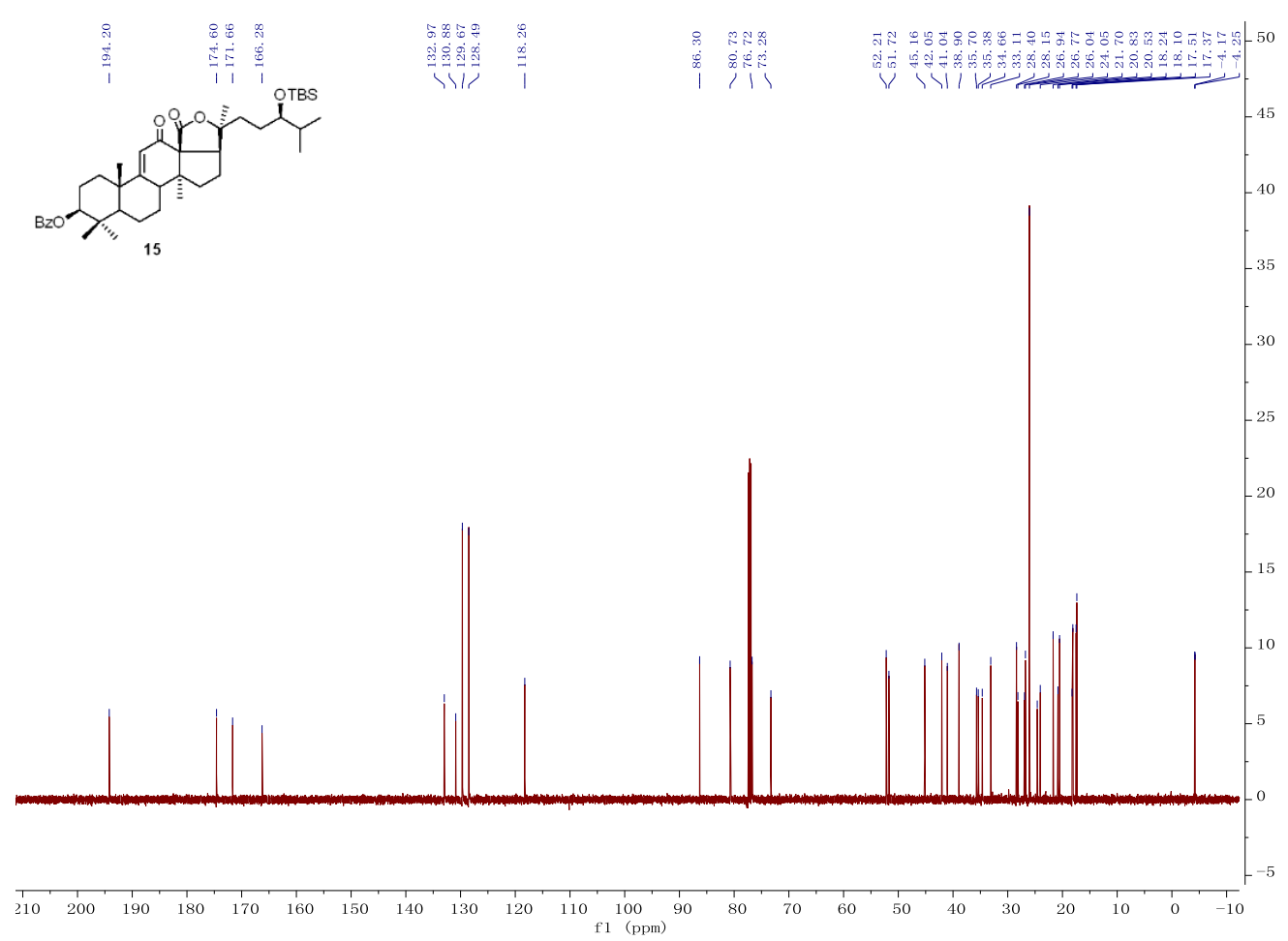

Figure S18. ${ }^{13} \mathrm{C}$ NMR spectrum of compound $15\left(125 \mathrm{MHz}, \mathrm{CDCl}_{3}\right)$ 


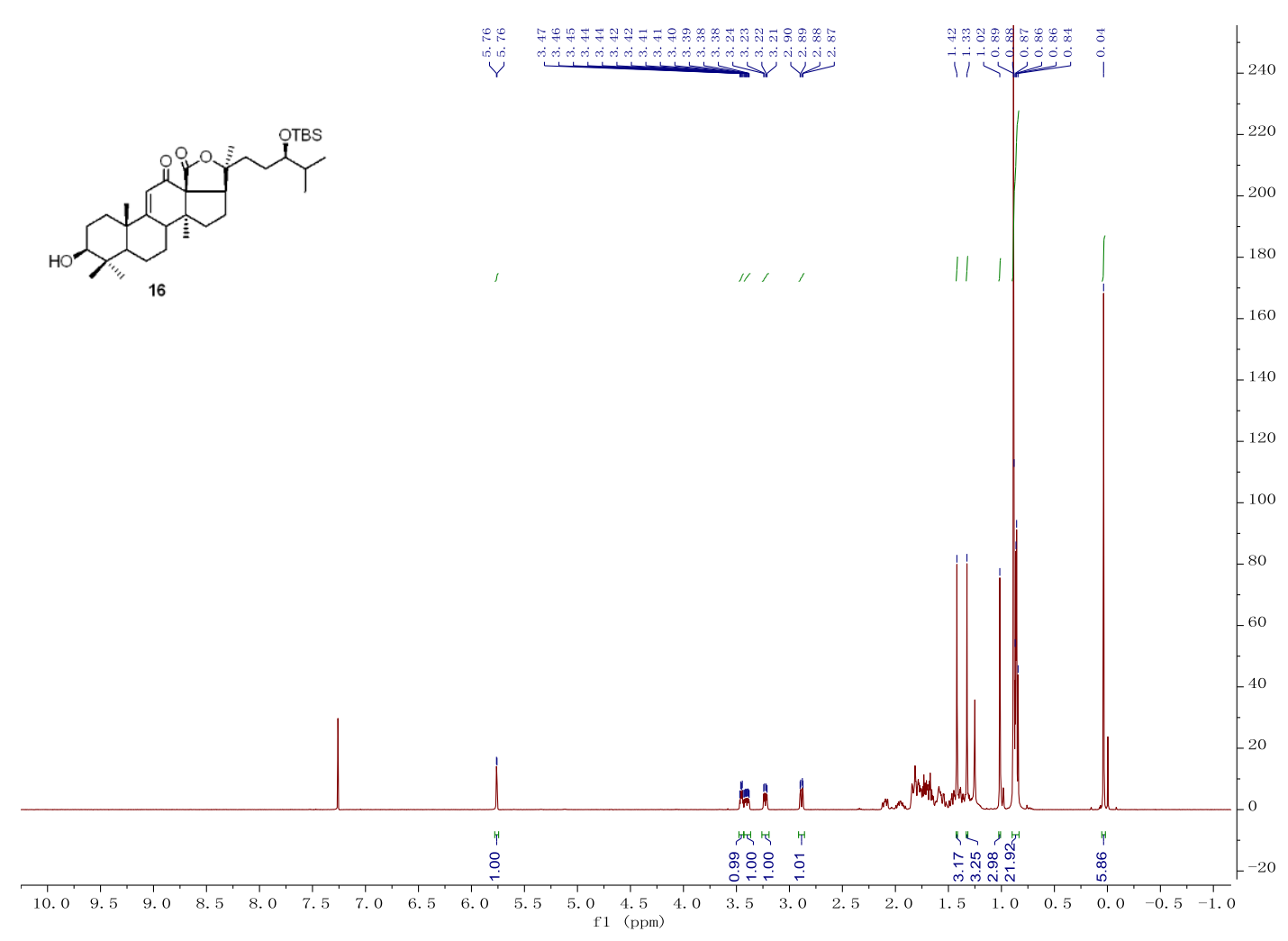

Figure S19. ${ }^{1} \mathrm{H}$ NMR spectrum of compound $16\left(500 \mathrm{MHz}, \mathrm{CDCl}_{3}\right)$

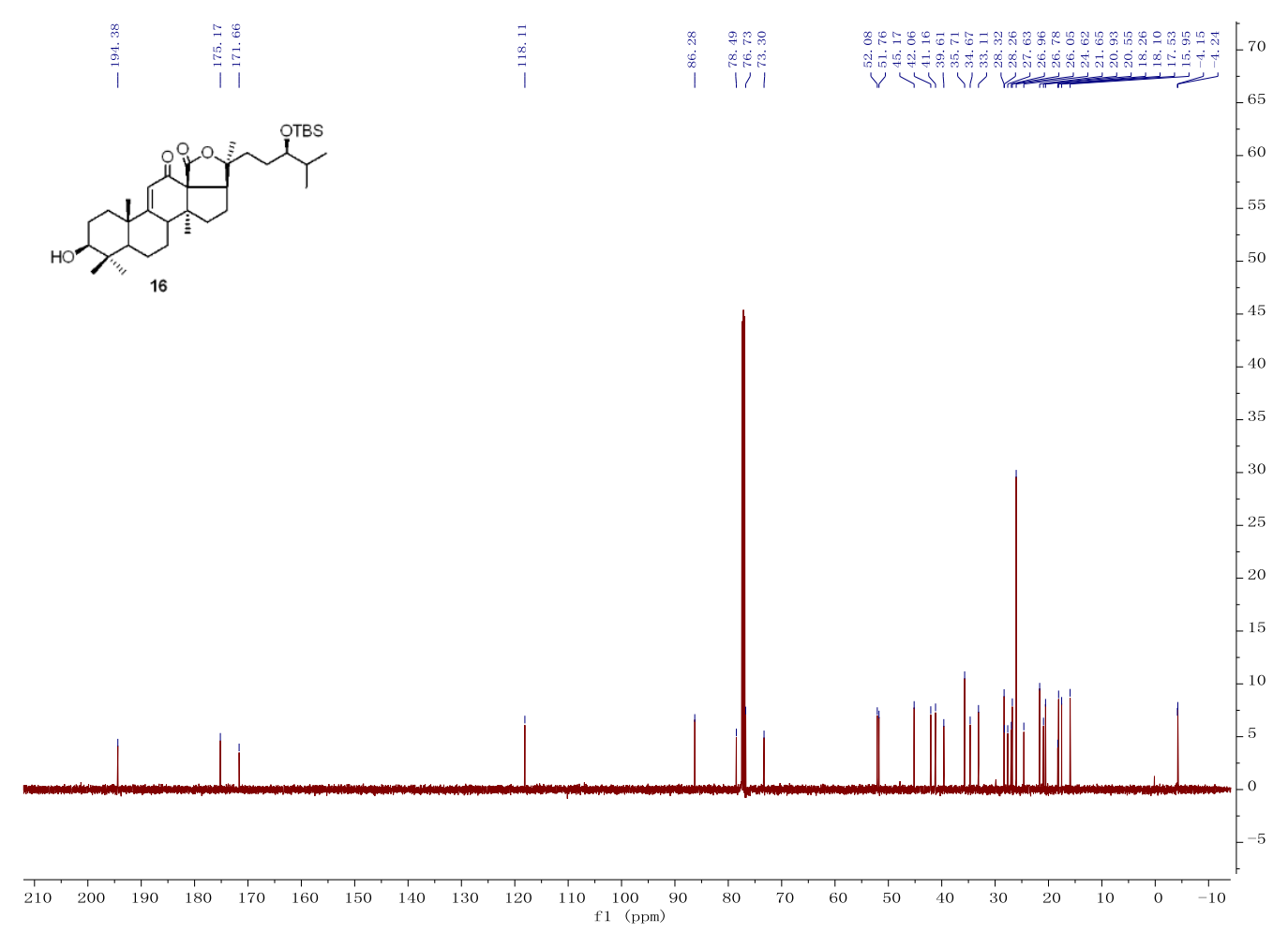

Figure S20. ${ }^{13} \mathrm{C}$ NMR spectrum of compound $16\left(125 \mathrm{MHz}, \mathrm{CDCl}_{3}\right)$ 


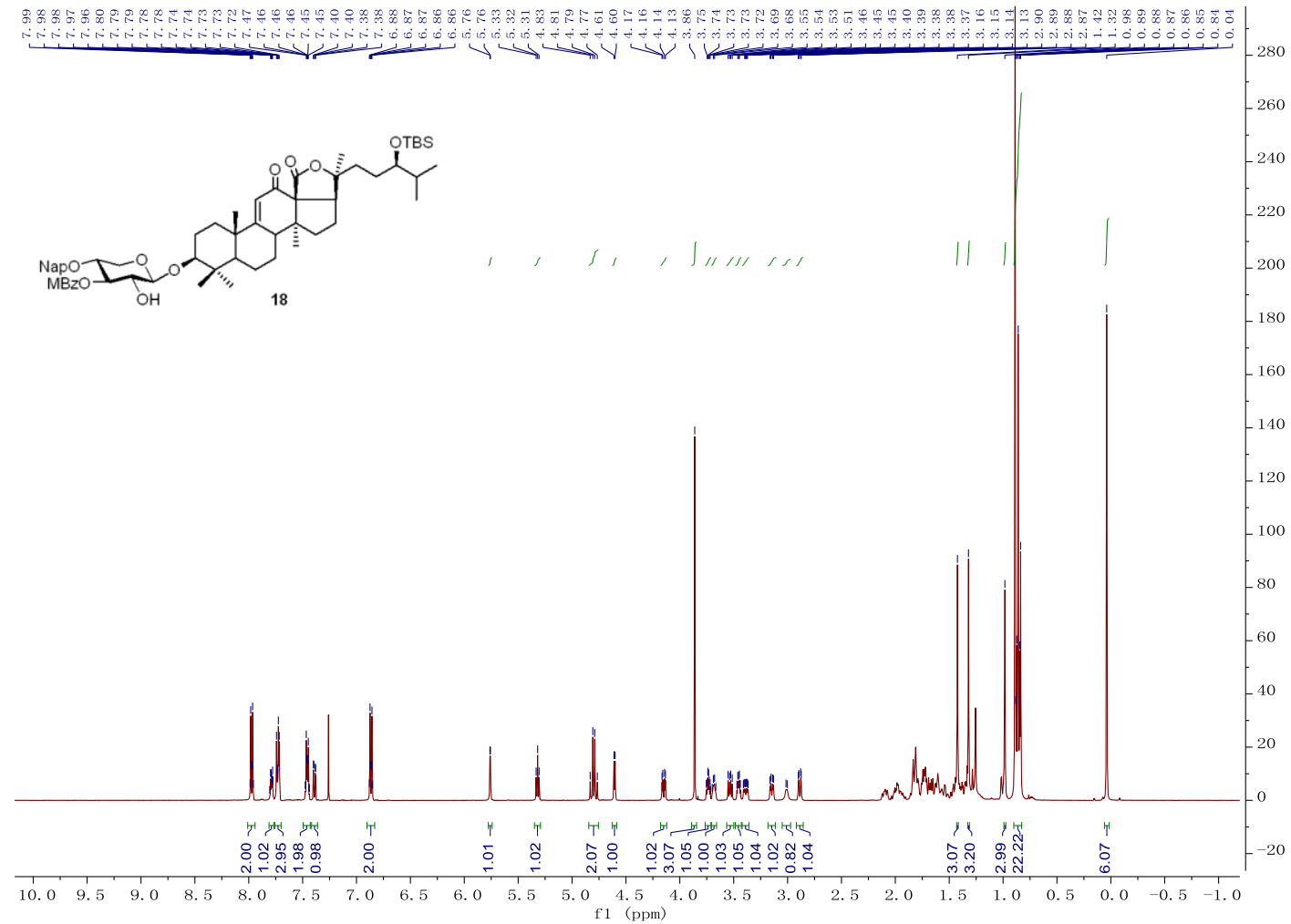

Figure S21. ${ }^{1} \mathrm{H}$ NMR spectrum of compound $18\left(500 \mathrm{MHz}, \mathrm{CDCl}_{3}\right)$

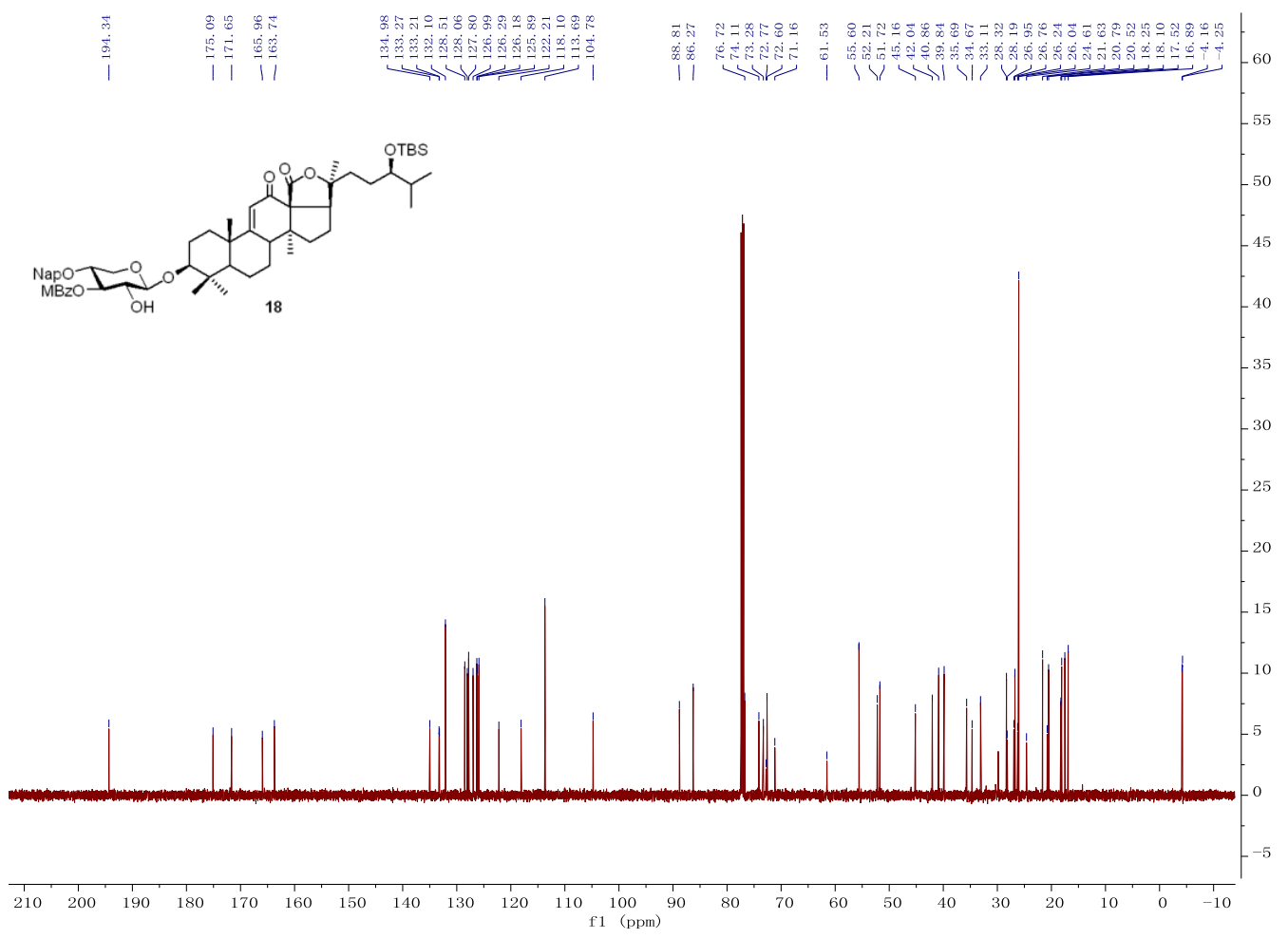

Figure S22. ${ }^{13} \mathrm{C}$ NMR spectrum of compound $18\left(125 \mathrm{MHz}, \mathrm{CDCl}_{3}\right)$ 


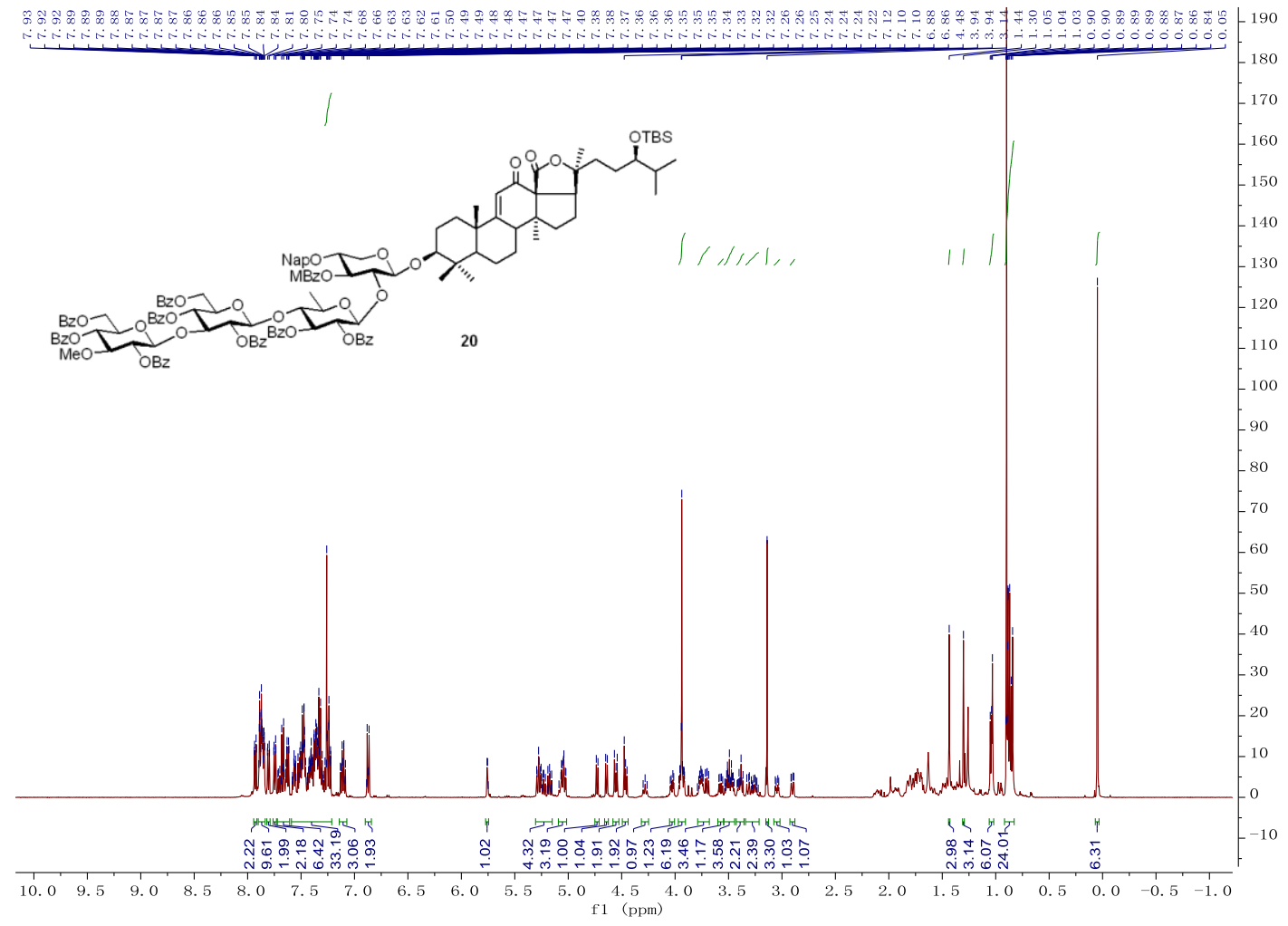

Figure S23. ${ }^{1} \mathrm{H}$ NMR spectrum of compound $20\left(500 \mathrm{MHz}, \mathrm{CDCl}_{3}\right)$

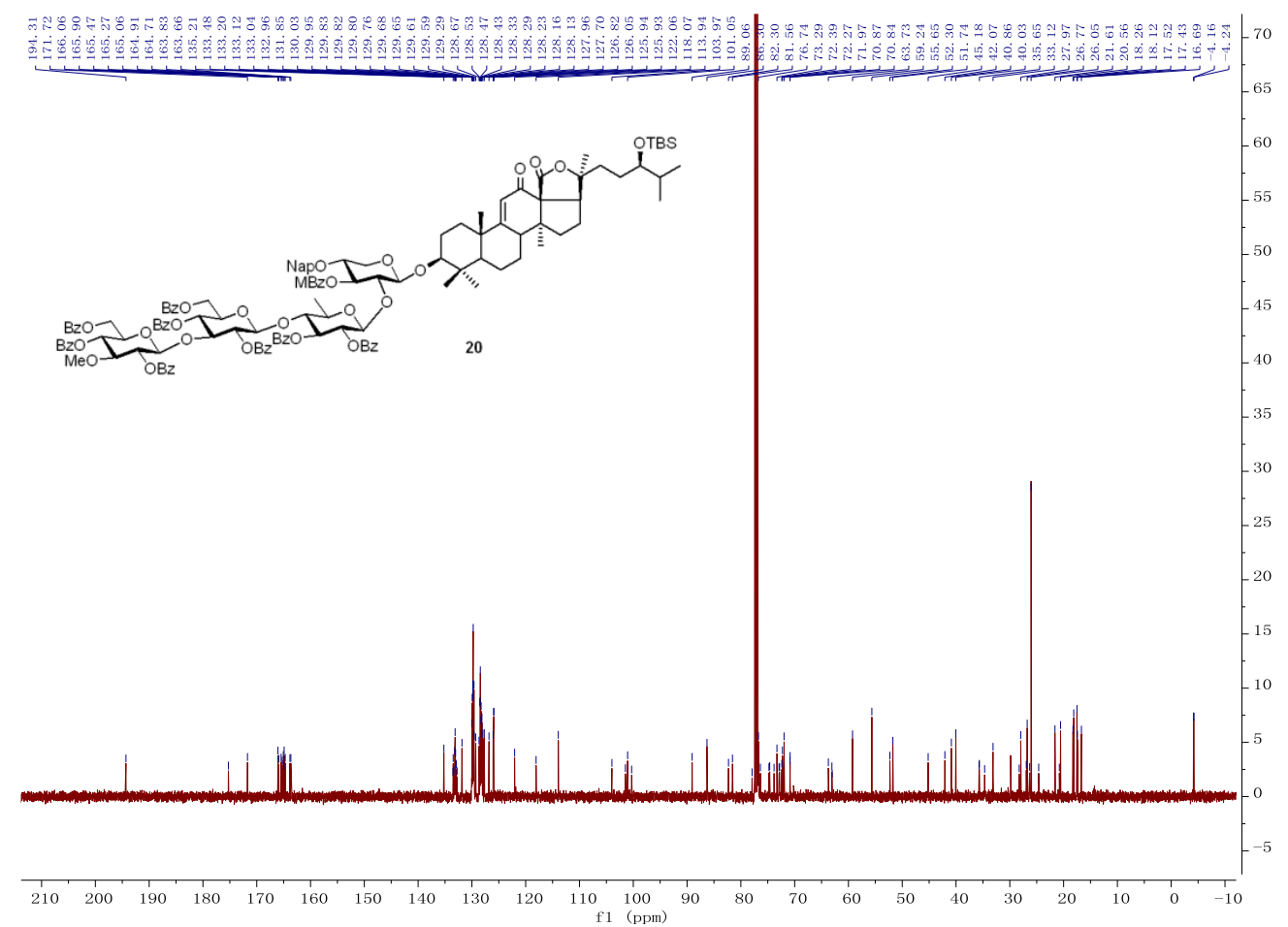

Figure S24. ${ }^{13} \mathrm{C}$ NMR spectrum of compound $20\left(125 \mathrm{MHz}, \mathrm{CDCl}_{3}\right)$ 


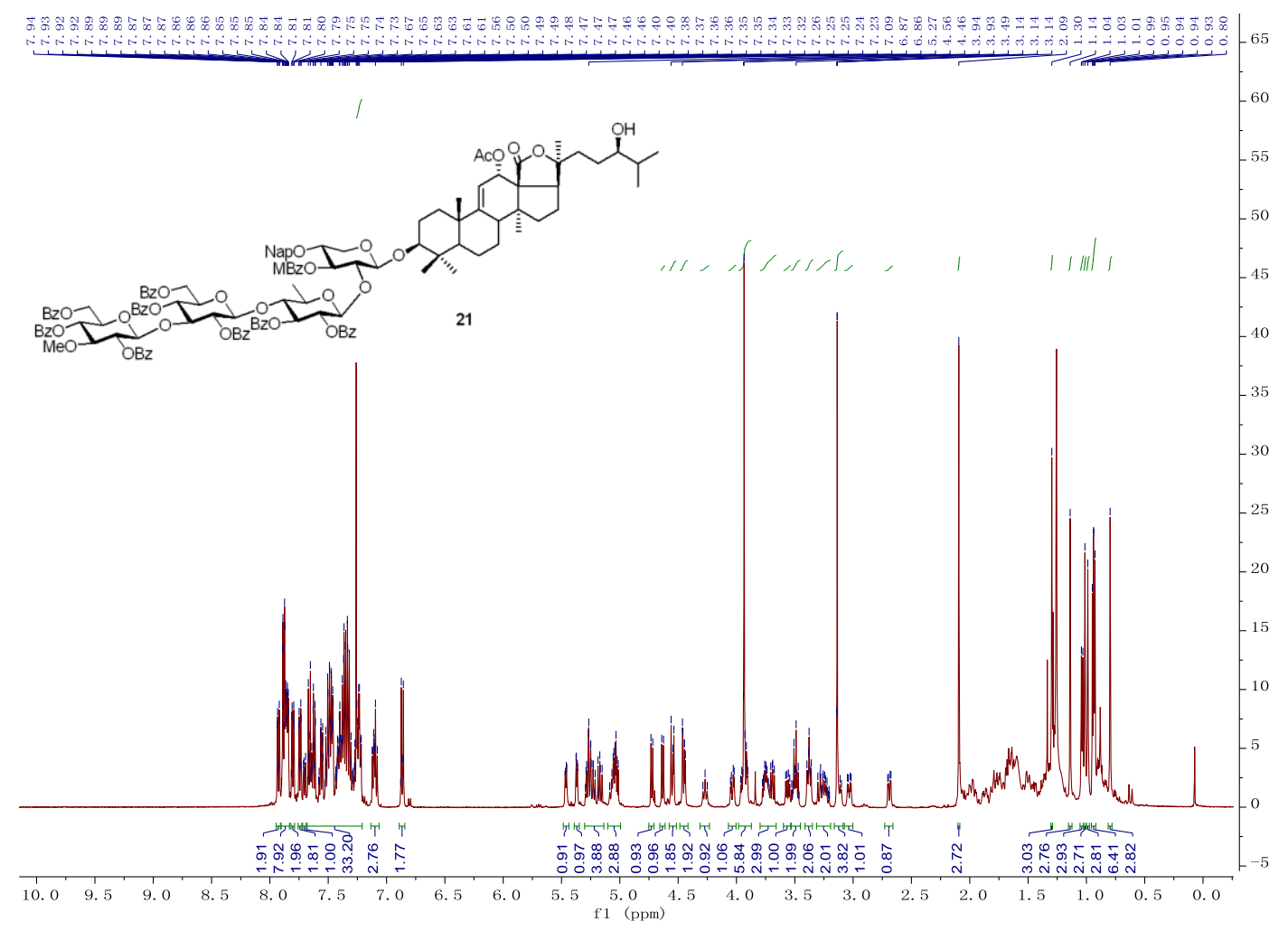

Figure S25. ${ }^{1} \mathrm{H}$ NMR spectrum of compound $21\left(500 \mathrm{MHz}, \mathrm{CDCl}_{3}\right)$

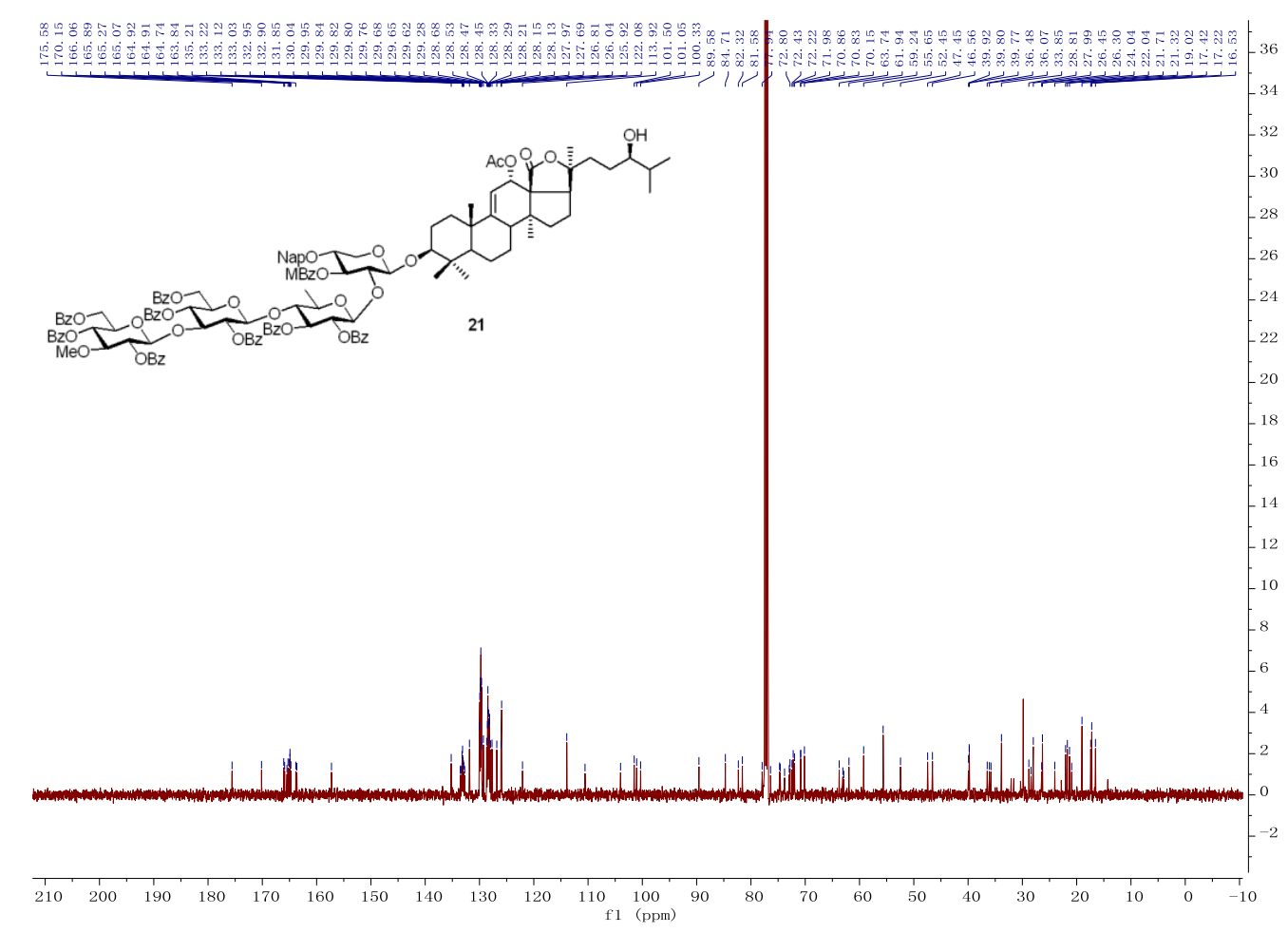

Figure S26. ${ }^{13} \mathrm{C}$ NMR spectrum of compound $21\left(125 \mathrm{MHz}, \mathrm{CDCl}_{3}\right)$ 


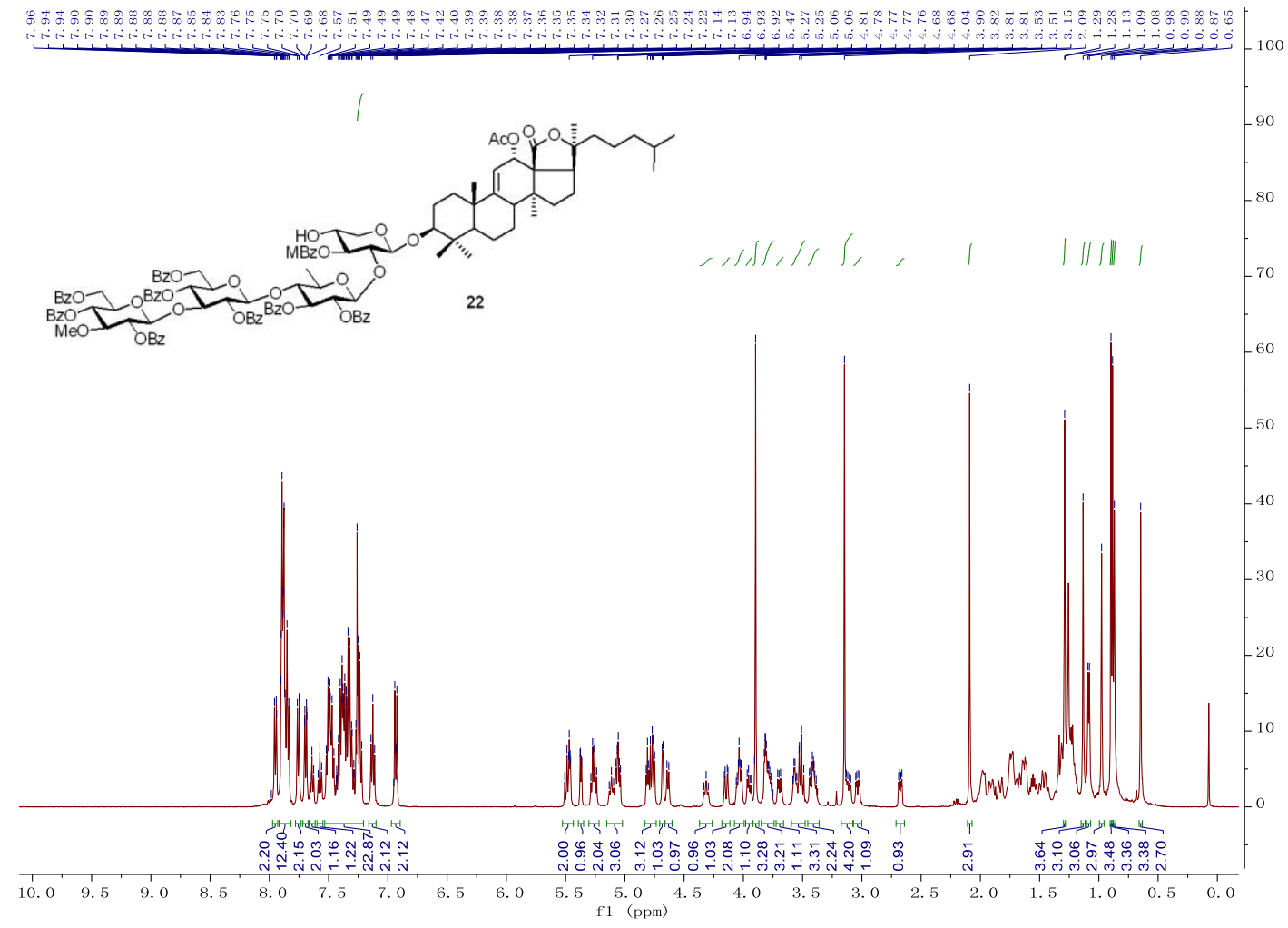

Figure S27. ${ }^{1} \mathrm{H}$ NMR spectrum of compound $22\left(500 \mathrm{MHz}, \mathrm{CDCl}_{3}\right)$

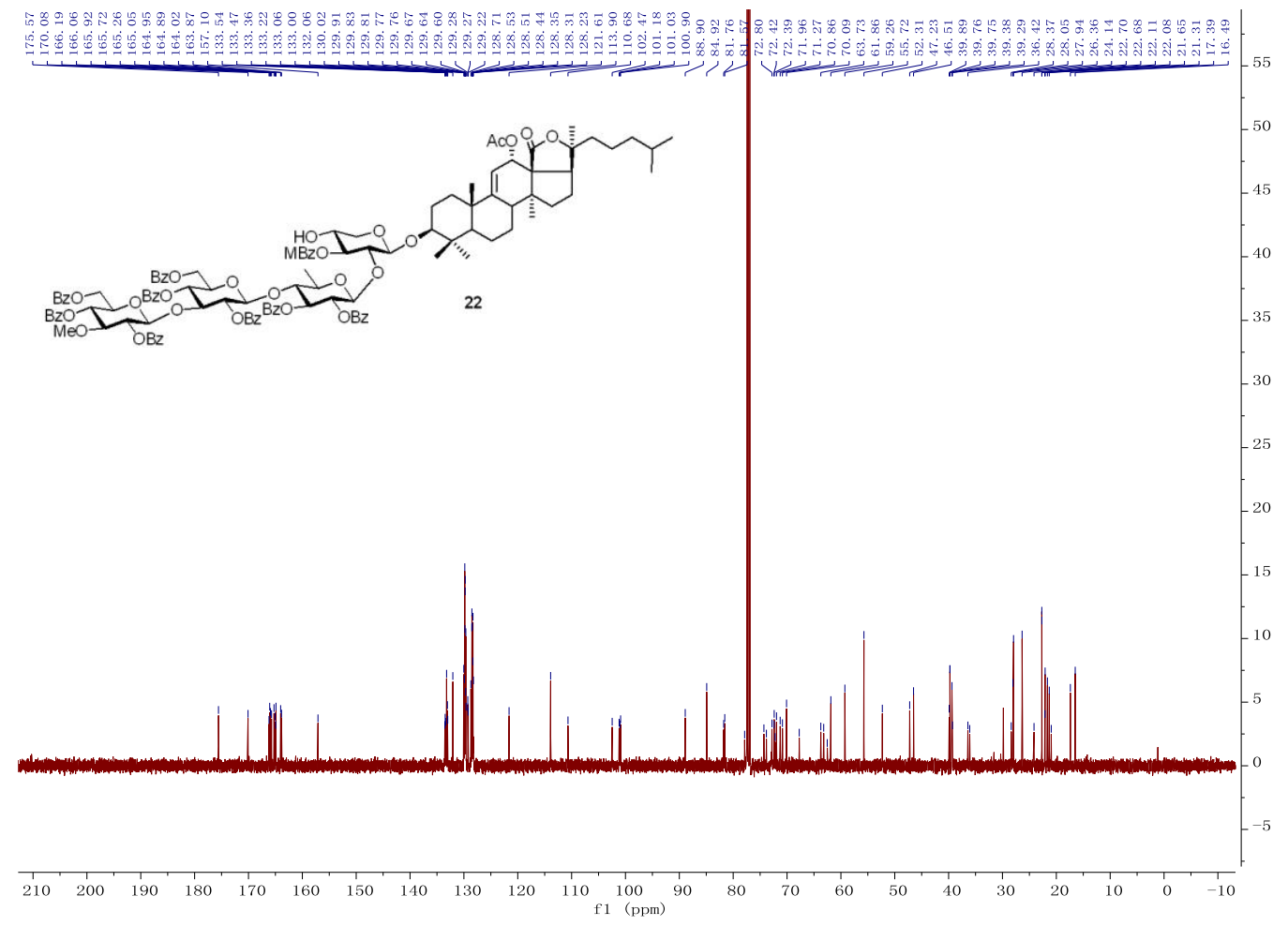

Figure S28. ${ }^{13} \mathrm{C}$ NMR spectrum of compound $22\left(125 \mathrm{MHz}, \mathrm{CDCl}_{3}\right)$ 


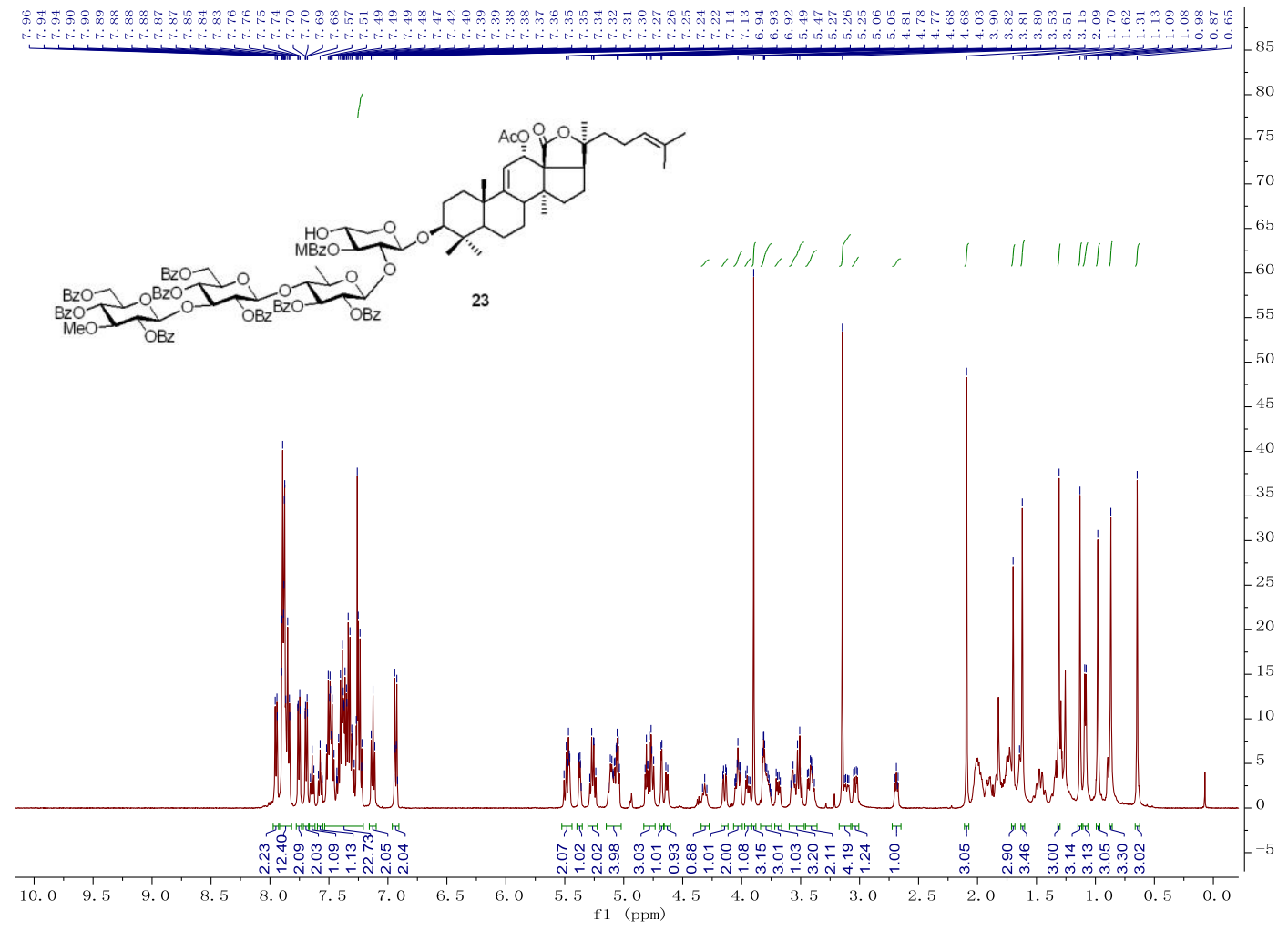

Figure S29. ${ }^{1} \mathrm{H}$ NMR spectrum of compound $23\left(500 \mathrm{MHz}, \mathrm{CDCl}_{3}\right)$

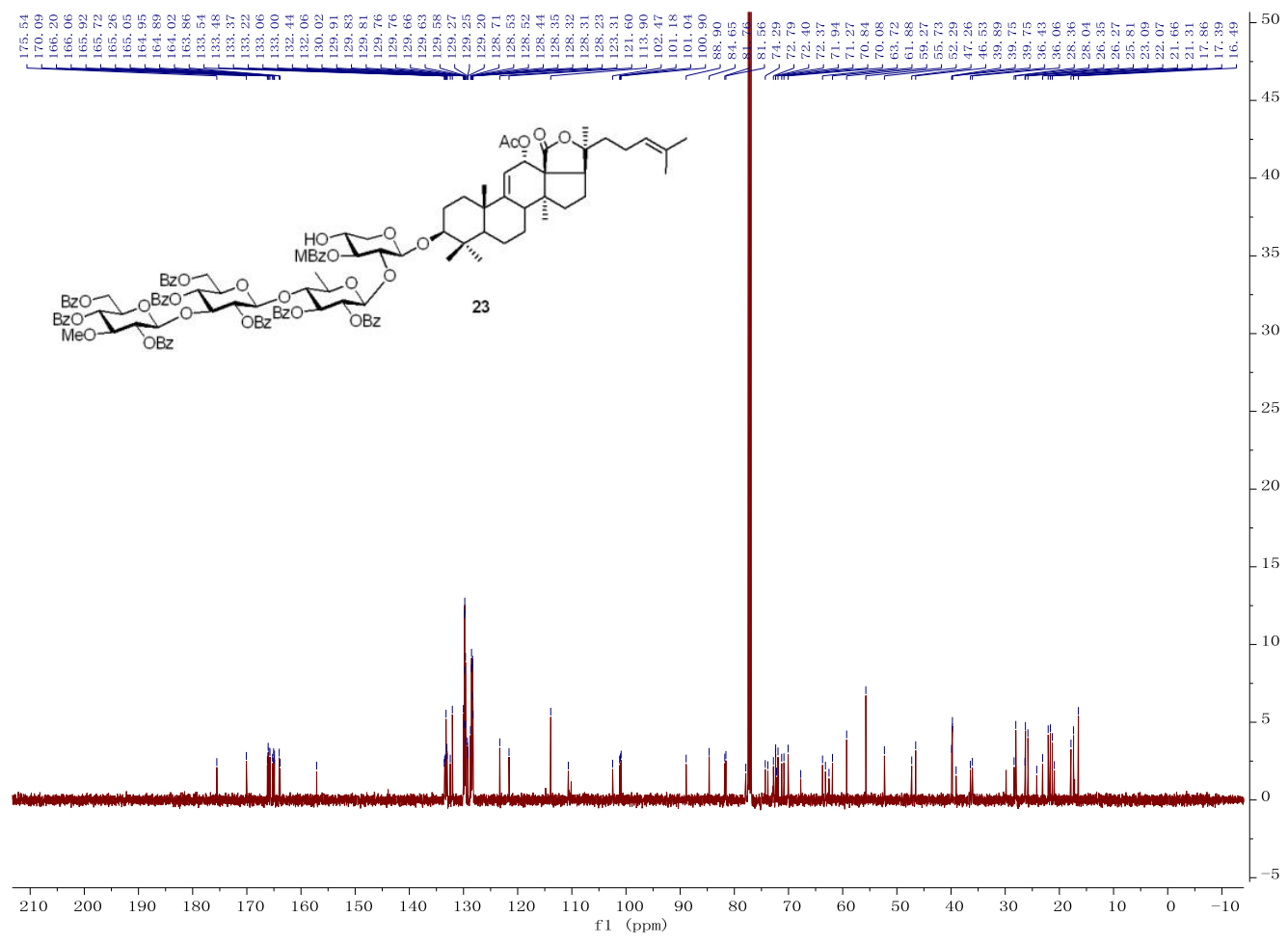

Figure S30. ${ }^{13} \mathrm{C}$ NMR spectrum of compound $23\left(125 \mathrm{MHz}, \mathrm{CDCl}_{3}\right)$ 


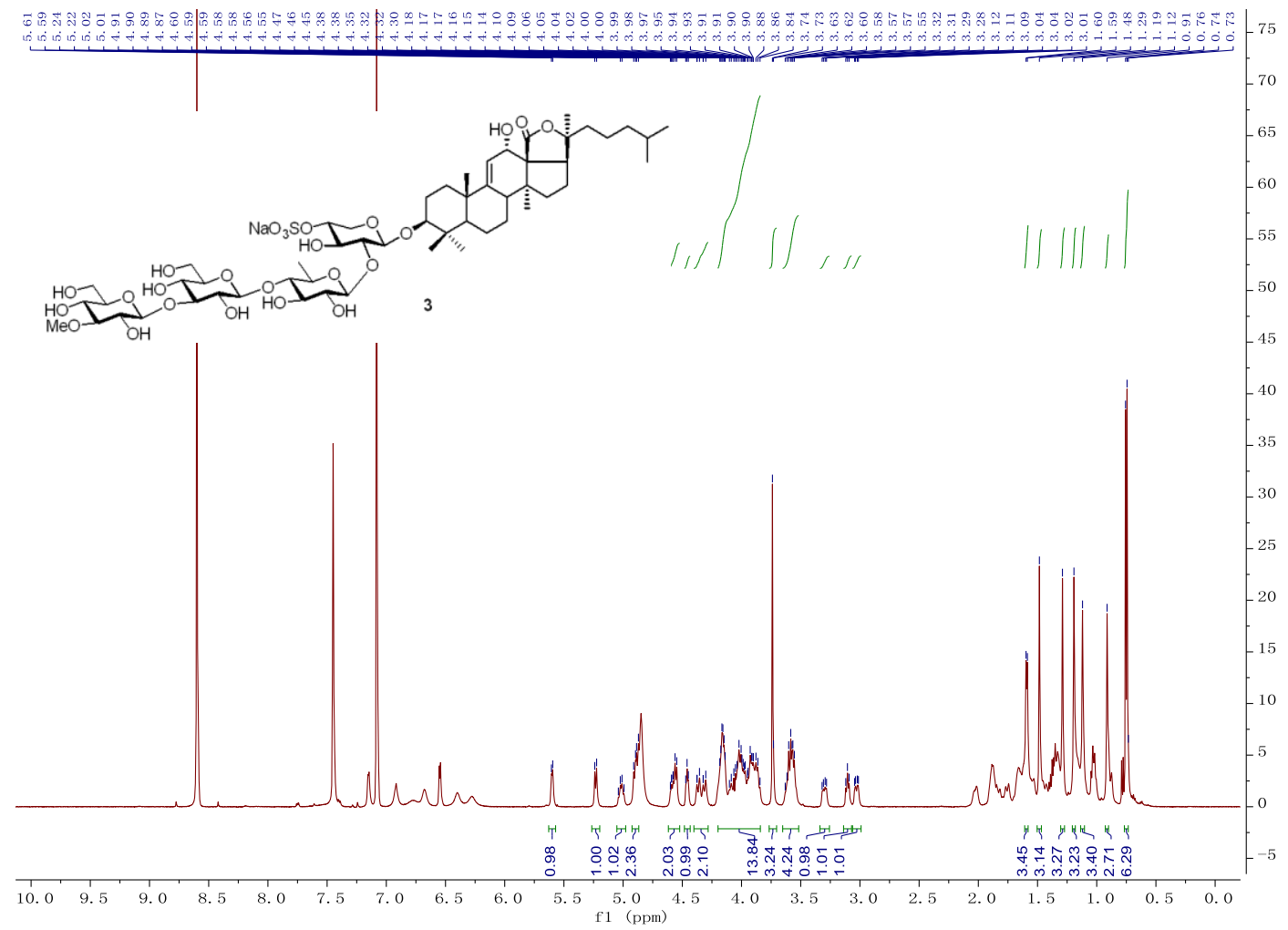

Figure S31. ${ }^{1} \mathrm{H}$ NMR spectrum of compound $3\left(500 \mathrm{MHz}, \mathrm{C}_{5} \mathrm{D}_{5} \mathrm{~N}\right)$

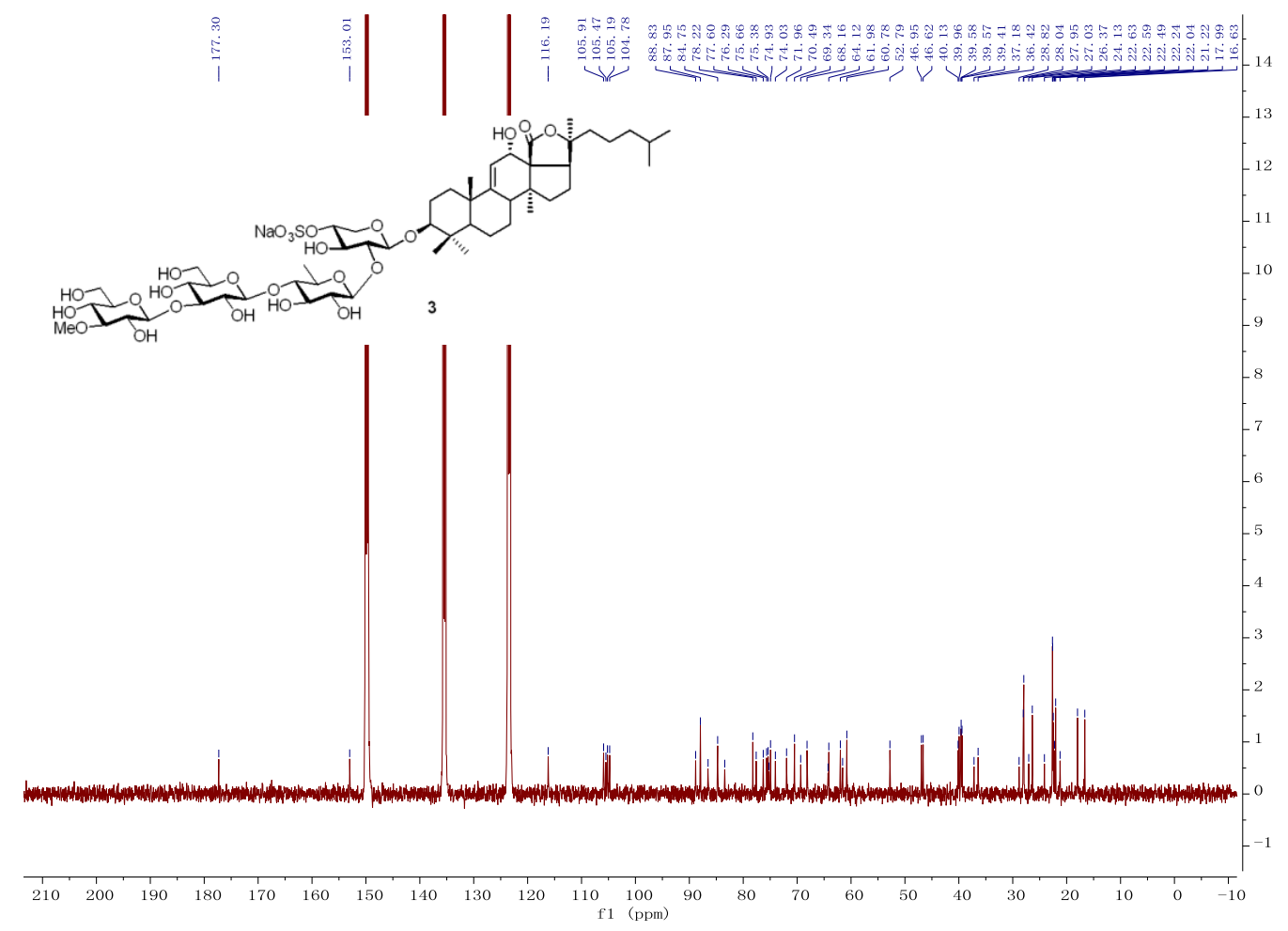

Figure S32. ${ }^{13} \mathrm{C}$ NMR spectrum of compound $3\left(125 \mathrm{MHz}, \mathrm{C}_{5} \mathrm{D}_{5} \mathrm{~N}\right)$ 


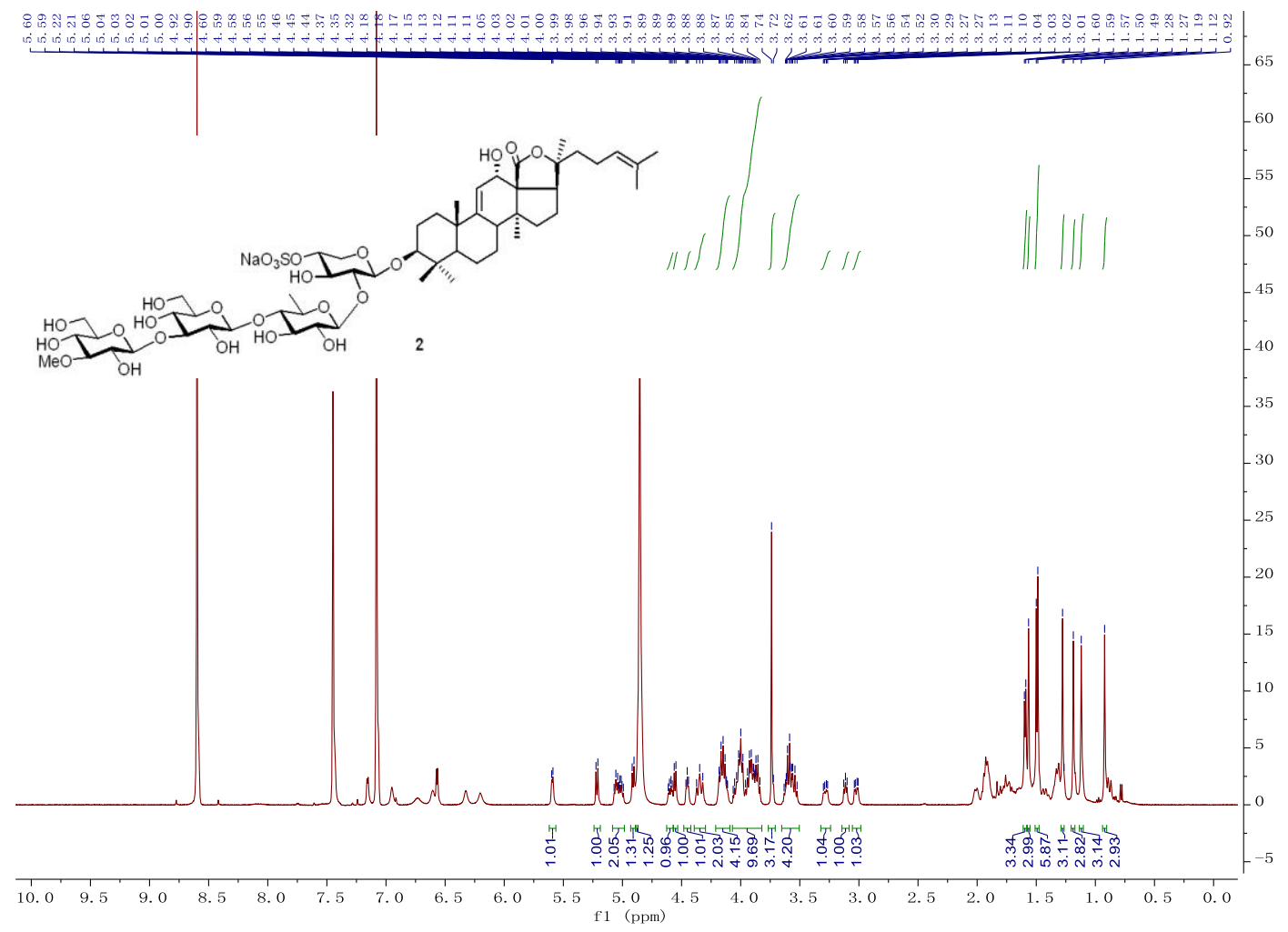

Figure S33. ${ }^{1} \mathrm{H}$ NMR spectrum of compound $2\left(500 \mathrm{MHz}, \mathrm{C}_{5} \mathrm{D}_{5} \mathrm{~N}\right)$

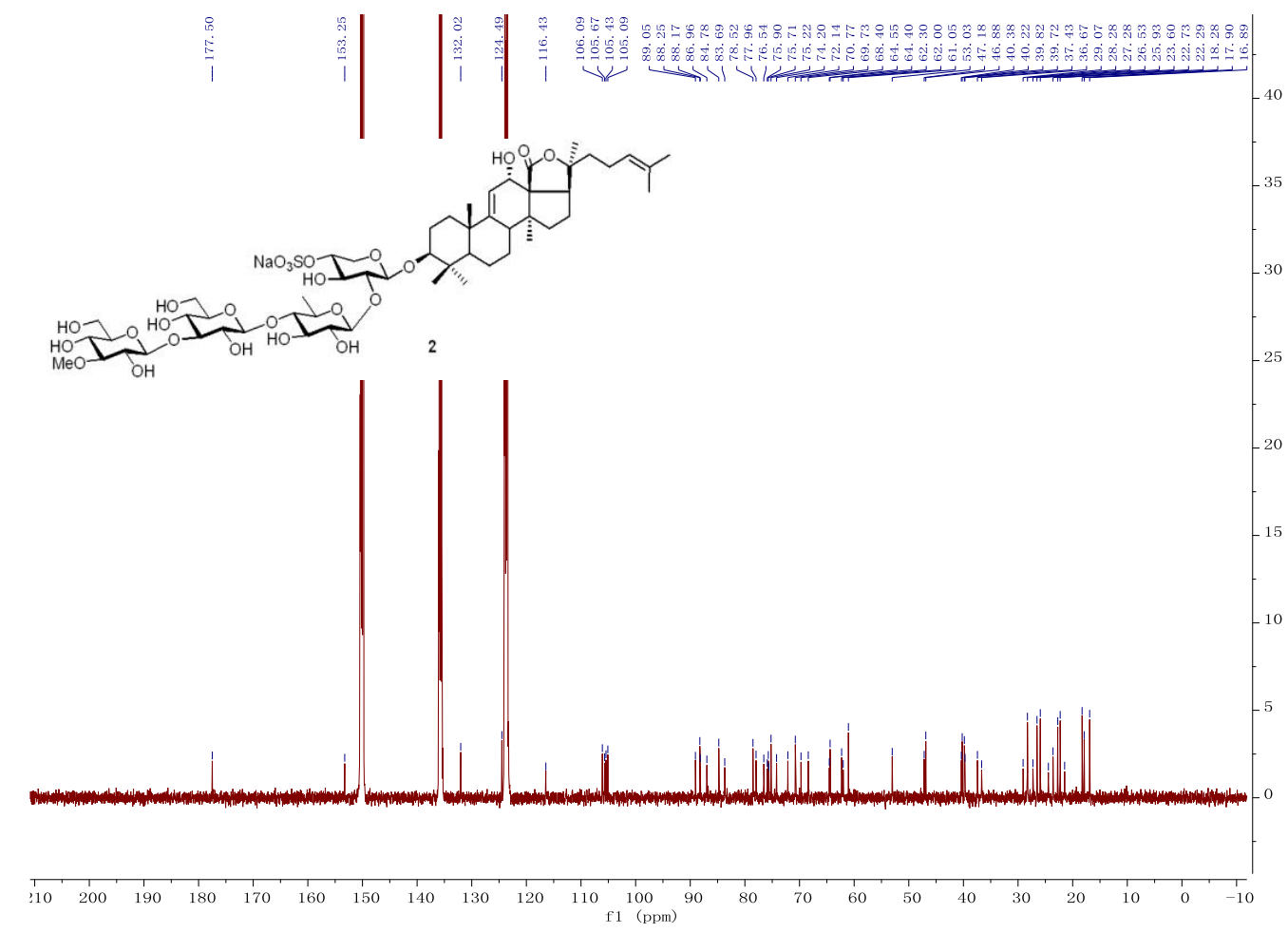

Figure S34. ${ }^{13} \mathrm{C}$ NMR spectrum of compound $2\left(125 \mathrm{MHz}, \mathrm{C}_{5} \mathrm{D}_{5} \mathrm{~N}\right)$ 


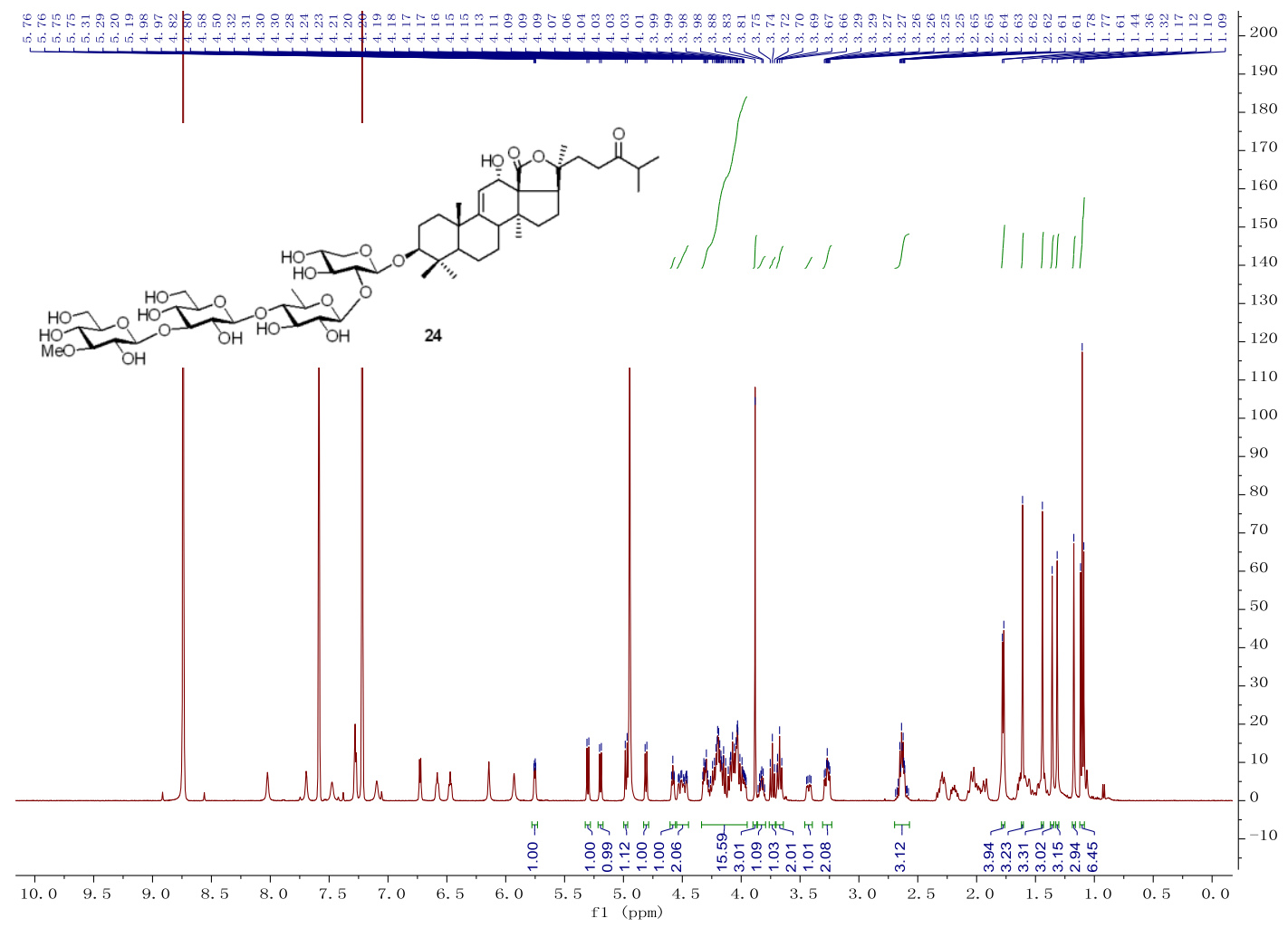

Figure S35. ${ }^{1} \mathrm{H}$ NMR spectrum of compound $24\left(500 \mathrm{MHz}, \mathrm{C}_{5} \mathrm{D}_{5} \mathrm{~N}\right)$

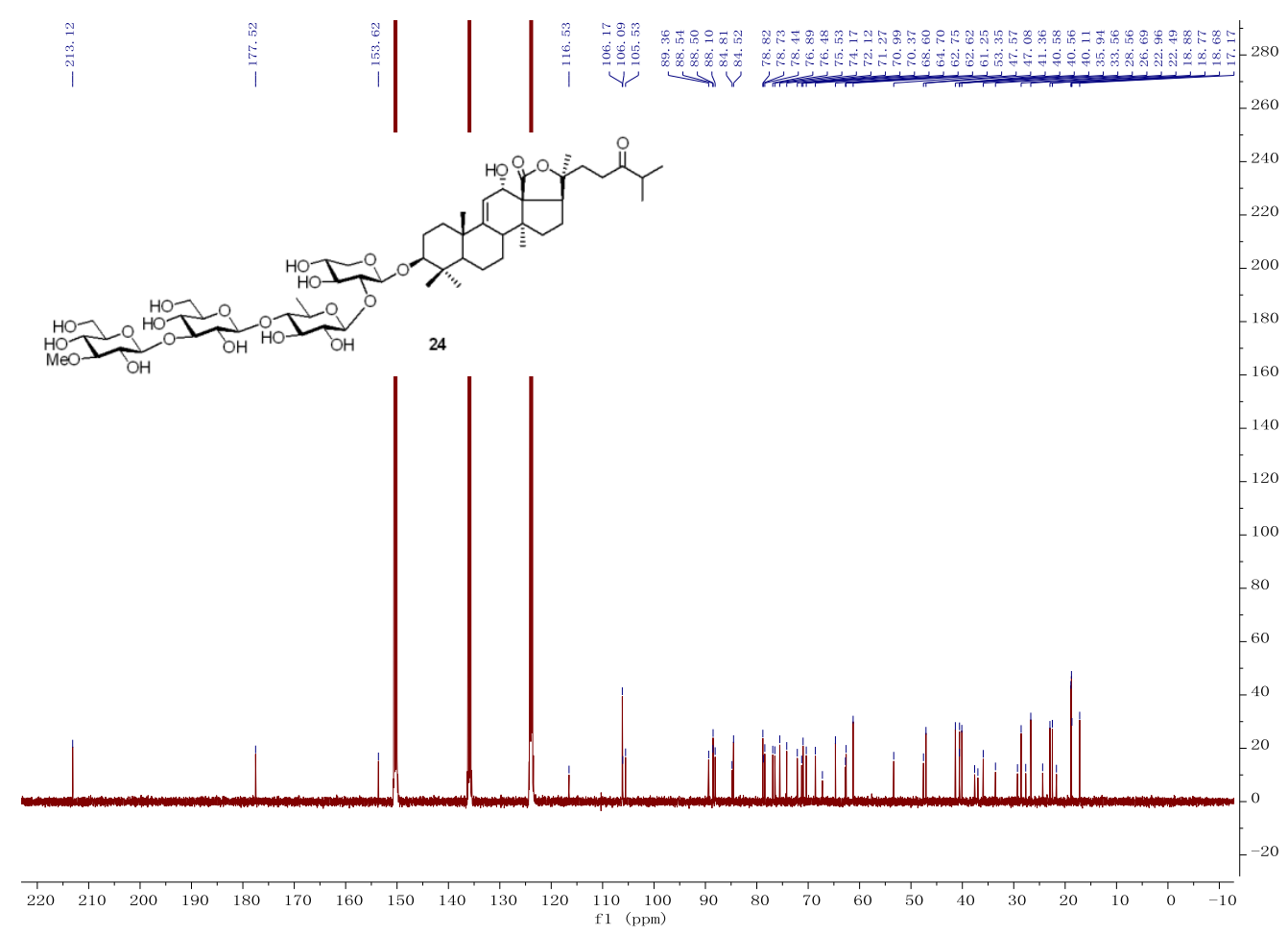

Figure S36. ${ }^{13} \mathrm{C}$ NMR spectrum of compound $24\left(125 \mathrm{MHz}, \mathrm{C}_{5} \mathrm{D}_{5} \mathrm{~N}\right)$ 


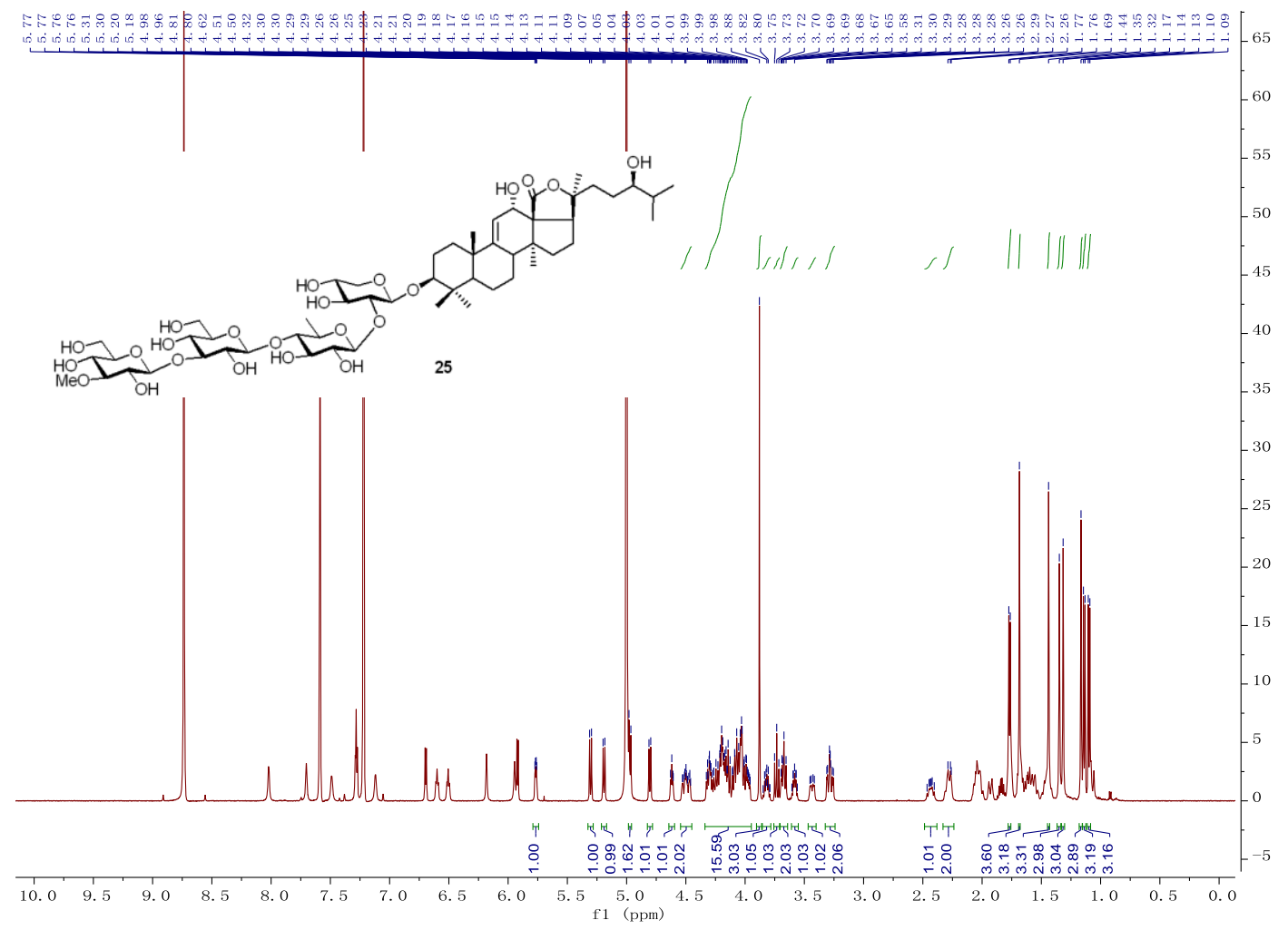

Figure S37. ${ }^{1} \mathrm{H}$ NMR spectrum of compound $25\left(500 \mathrm{MHz}, \mathrm{C}_{5} \mathrm{D}_{5} \mathrm{~N}\right)$

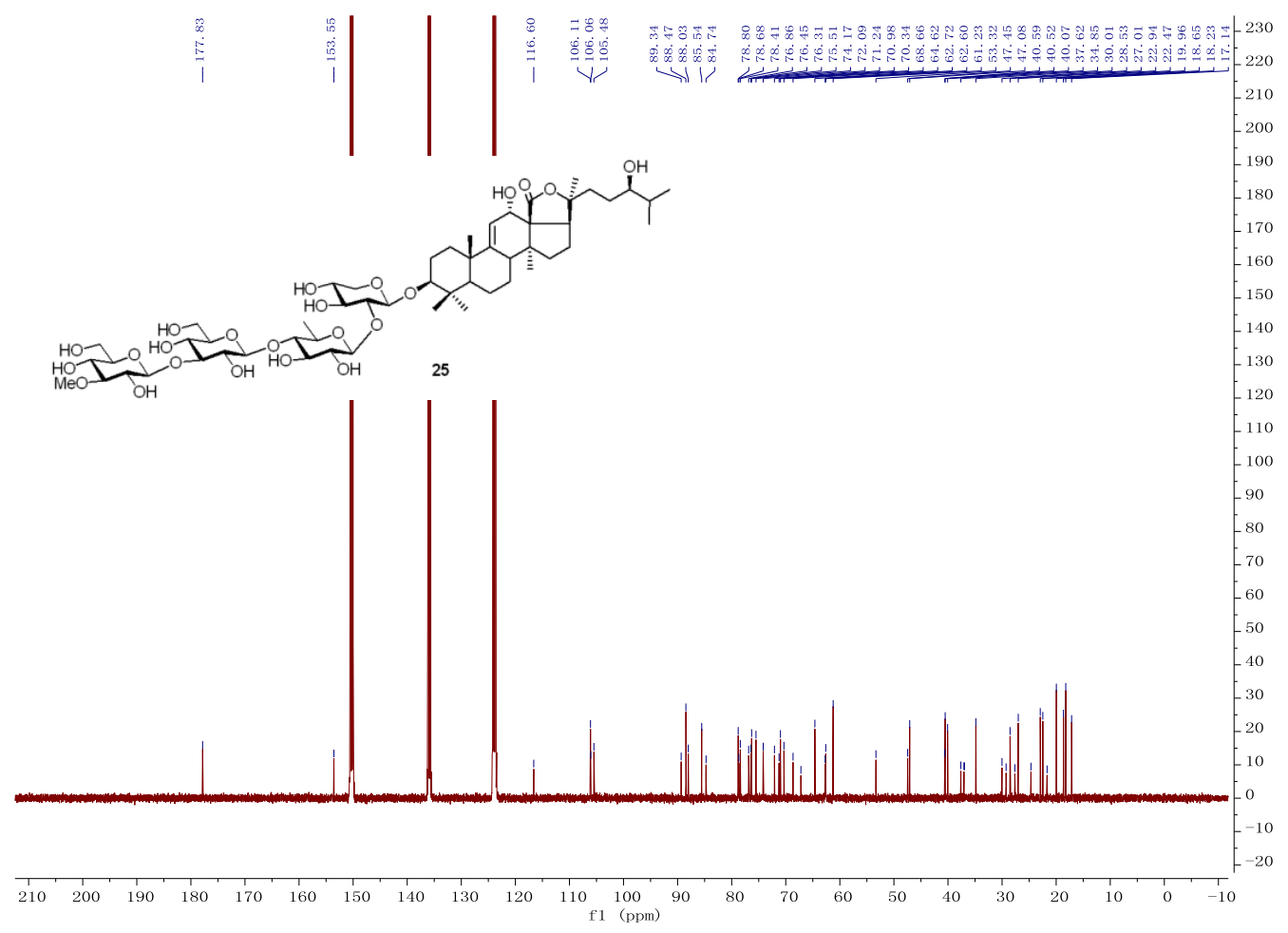

Figure S38. ${ }^{13} \mathrm{C}$ NMR spectrum of compound $25\left(125 \mathrm{MHz}, \mathrm{C}_{5} \mathrm{D}_{5} \mathrm{~N}\right)$ 


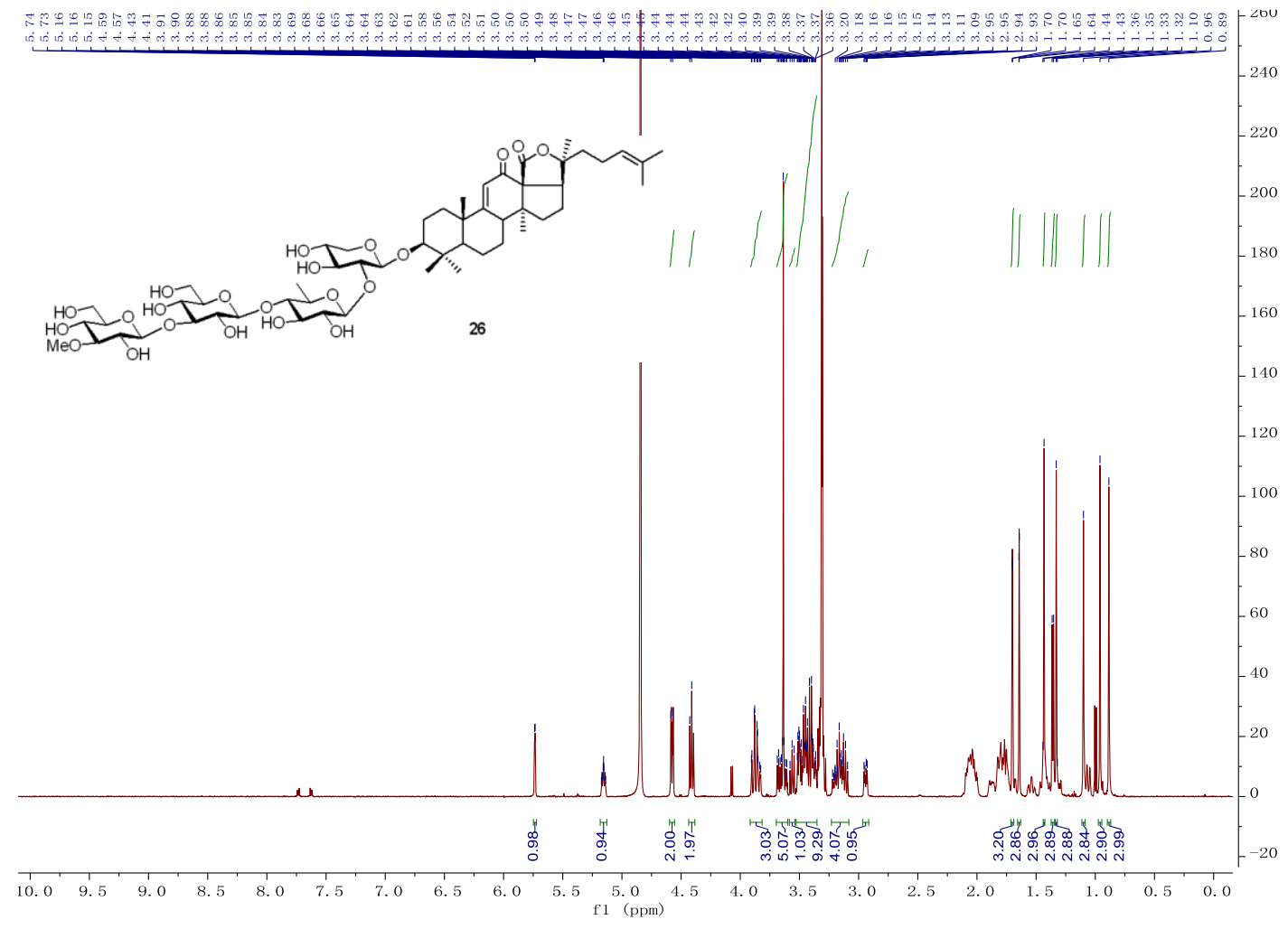

Figure S39. ${ }^{1} \mathrm{H}$ NMR spectrum of compound $26\left(500 \mathrm{MHz}, \mathrm{CD}_{3} \mathrm{OD}\right)$

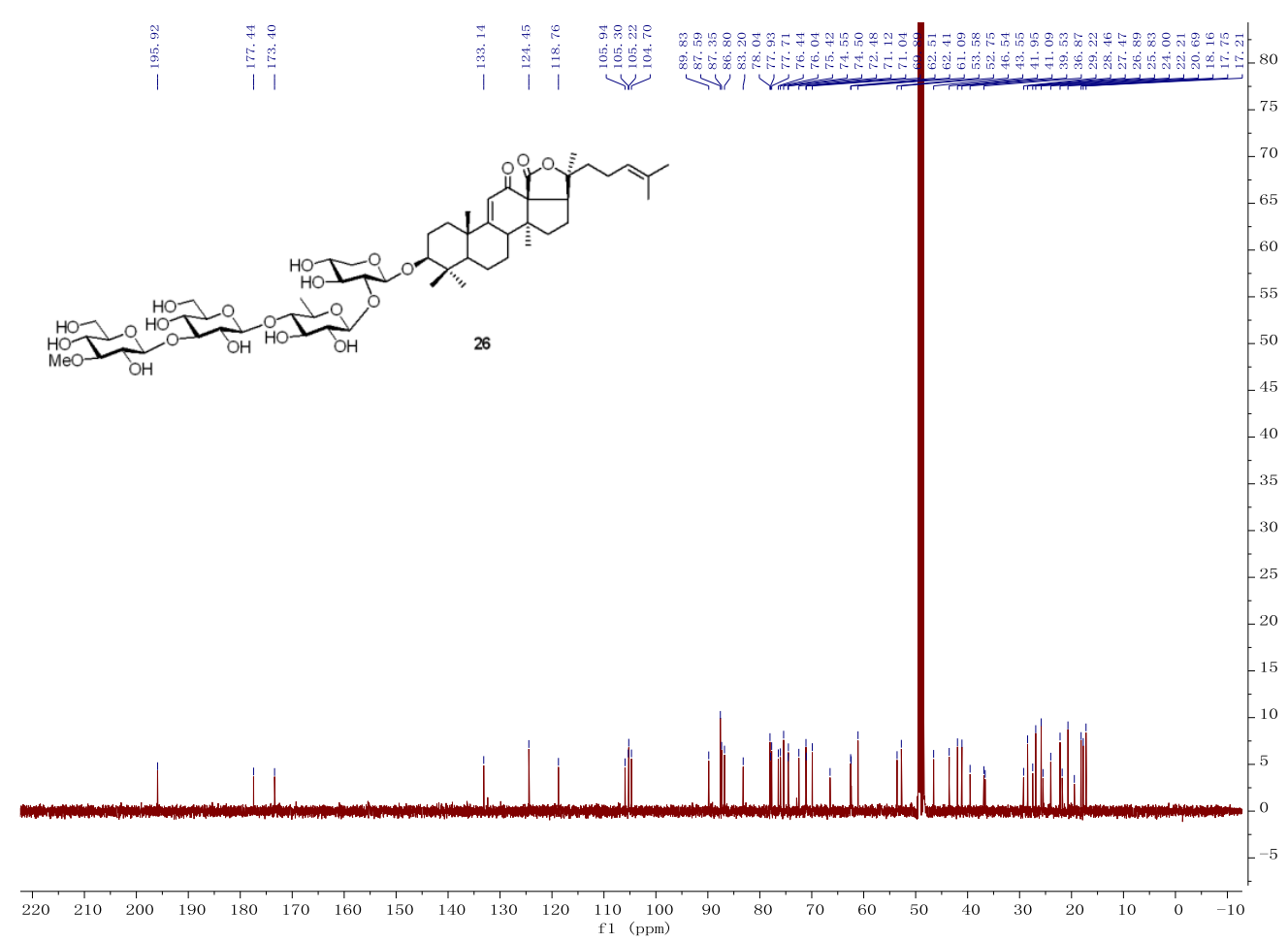

Figure S40. ${ }^{13} \mathrm{C}$ NMR spectrum of compound $26\left(125 \mathrm{MHz}, \mathrm{CD}_{3} \mathrm{OD}\right)$ 


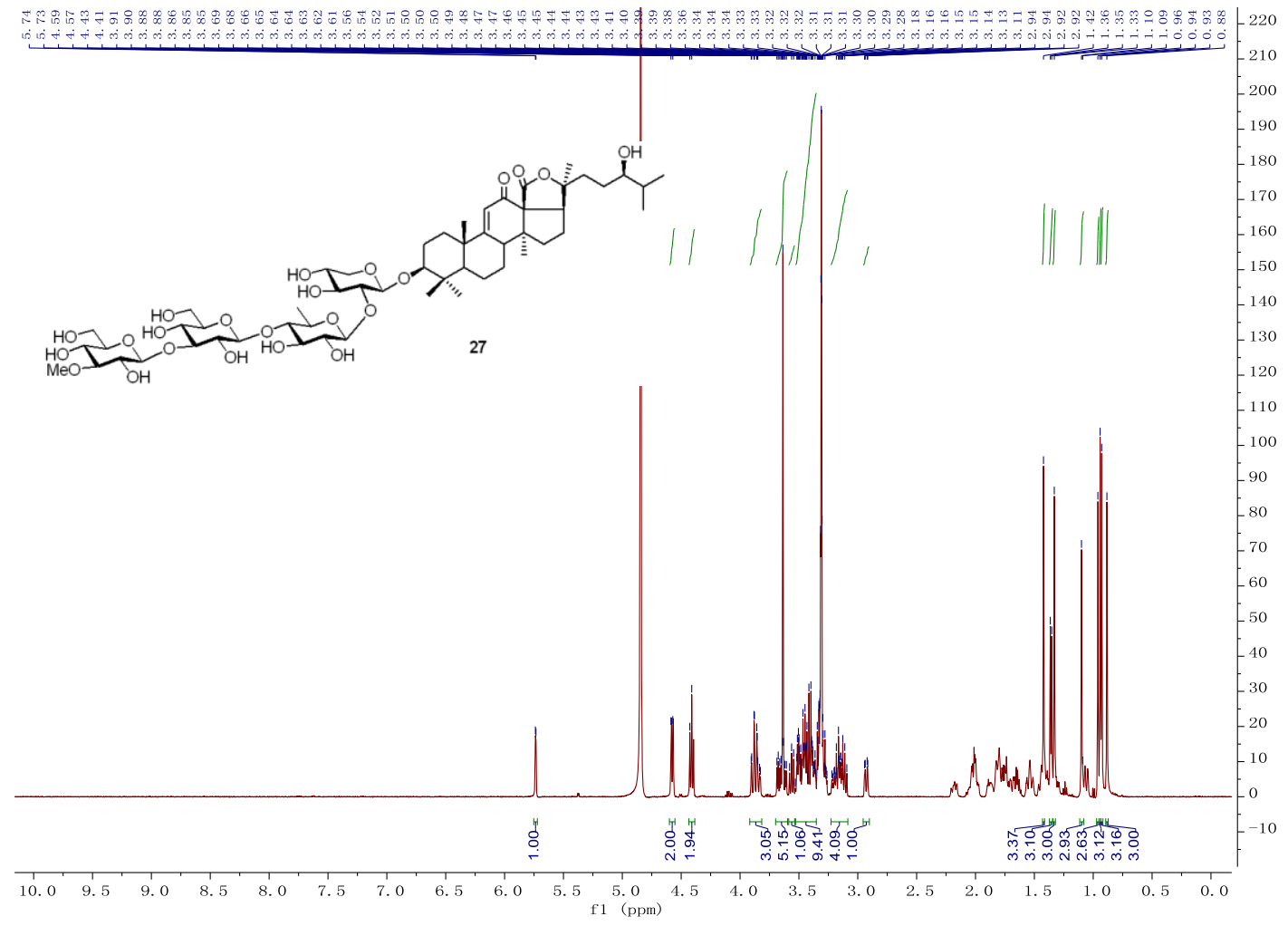

Figure S41. ${ }^{1} \mathrm{H}$ NMR spectrum of compound 27 (500 MHz, CD 3 OD)

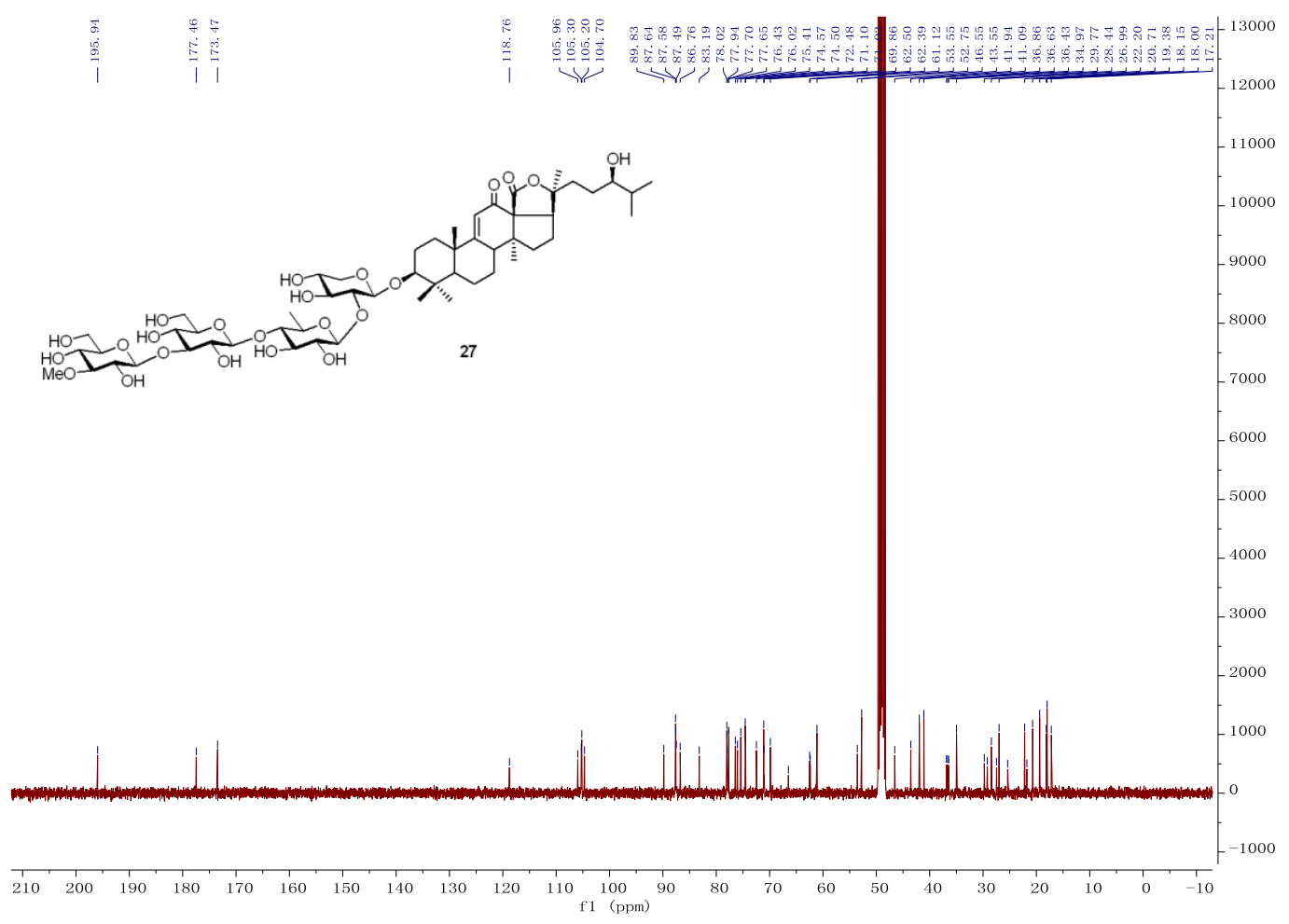

Figure S42. ${ }^{13} \mathrm{C}$ NMR spectrum of compound $27\left(125 \mathrm{MHz}, \mathrm{CD}_{3} \mathrm{OD}\right)$ 


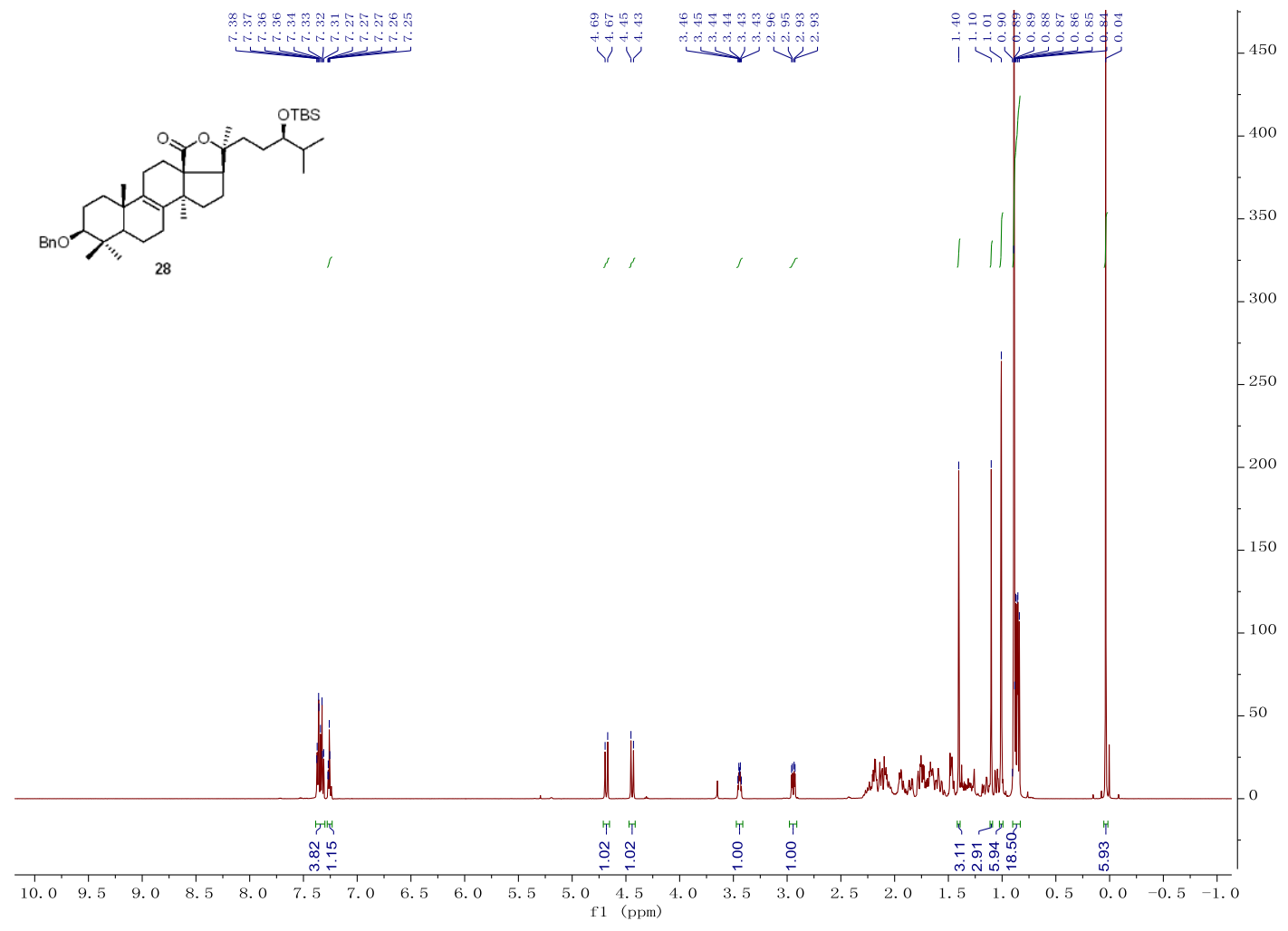

Figure S43. ${ }^{1} \mathrm{H}$ NMR spectrum of compound $28\left(500 \mathrm{MHz}, \mathrm{CDCl}_{3}\right)$

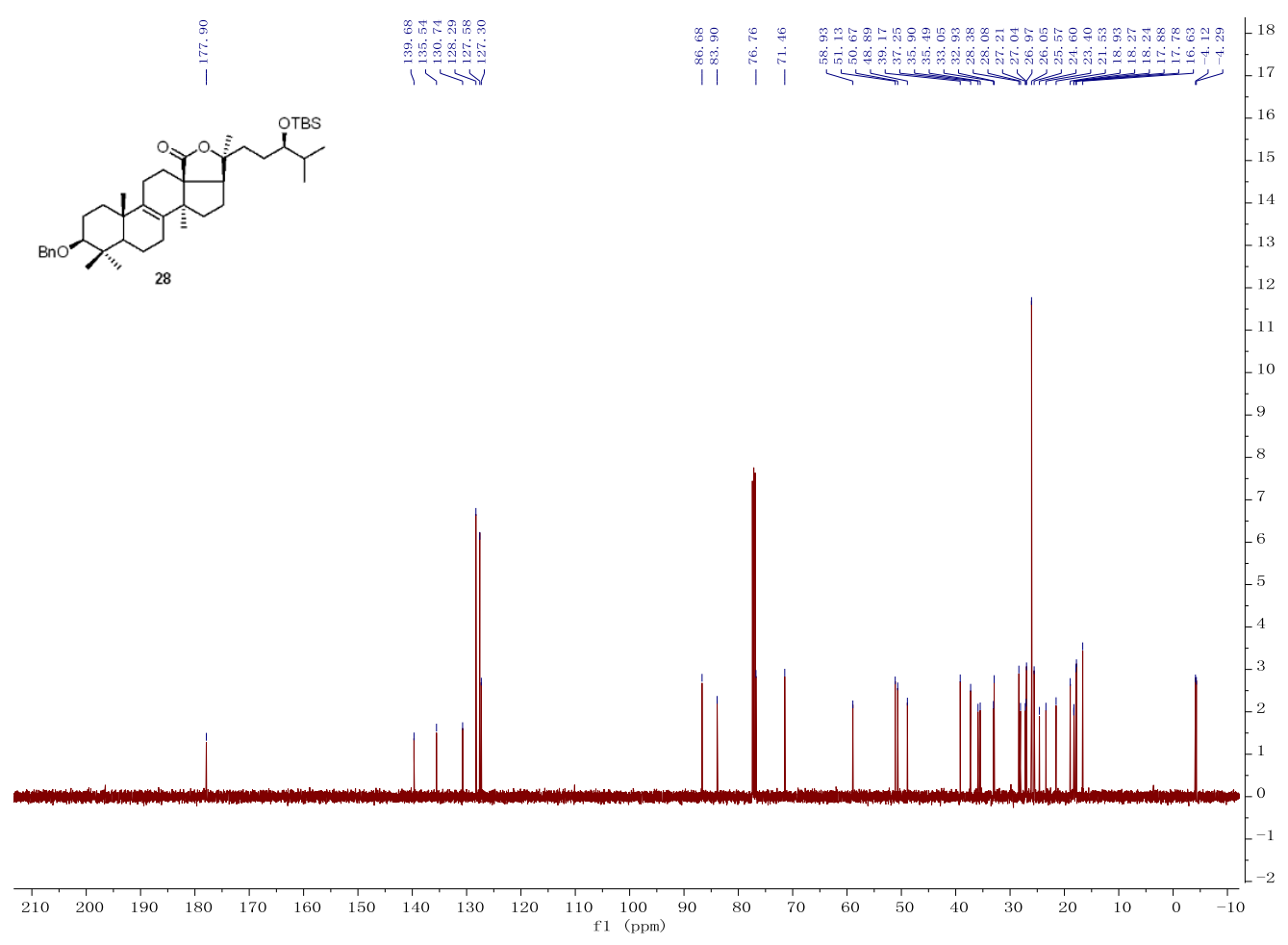

Figure S44. ${ }^{13} \mathrm{C}$ NMR spectrum of compound $28\left(125 \mathrm{MHz}, \mathrm{CDCl}_{3}\right)$ 


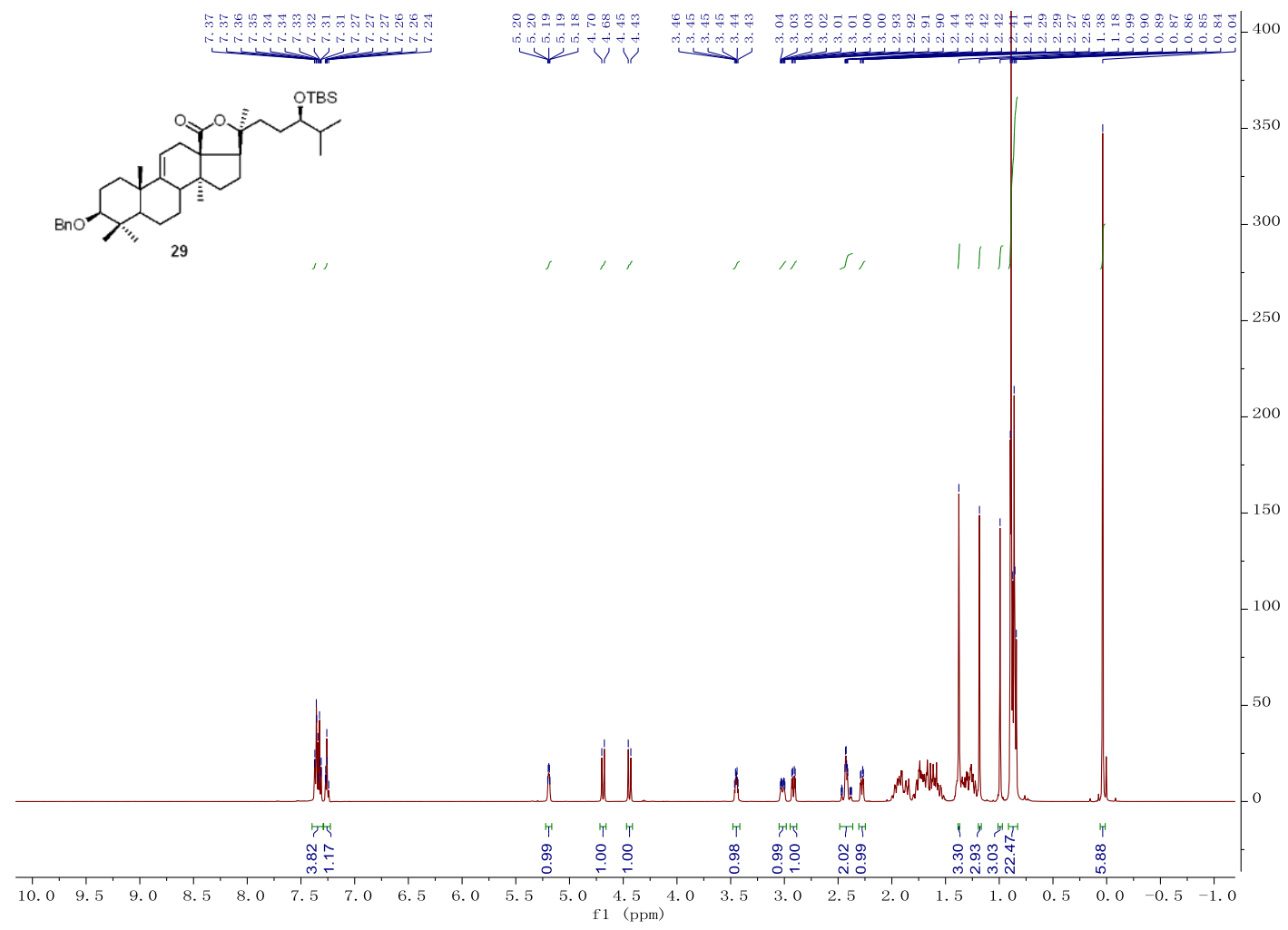

Figure S45. ${ }^{1} \mathrm{H}$ NMR spectrum of compound $29\left(500 \mathrm{MHz}, \mathrm{CDCl}_{3}\right)$

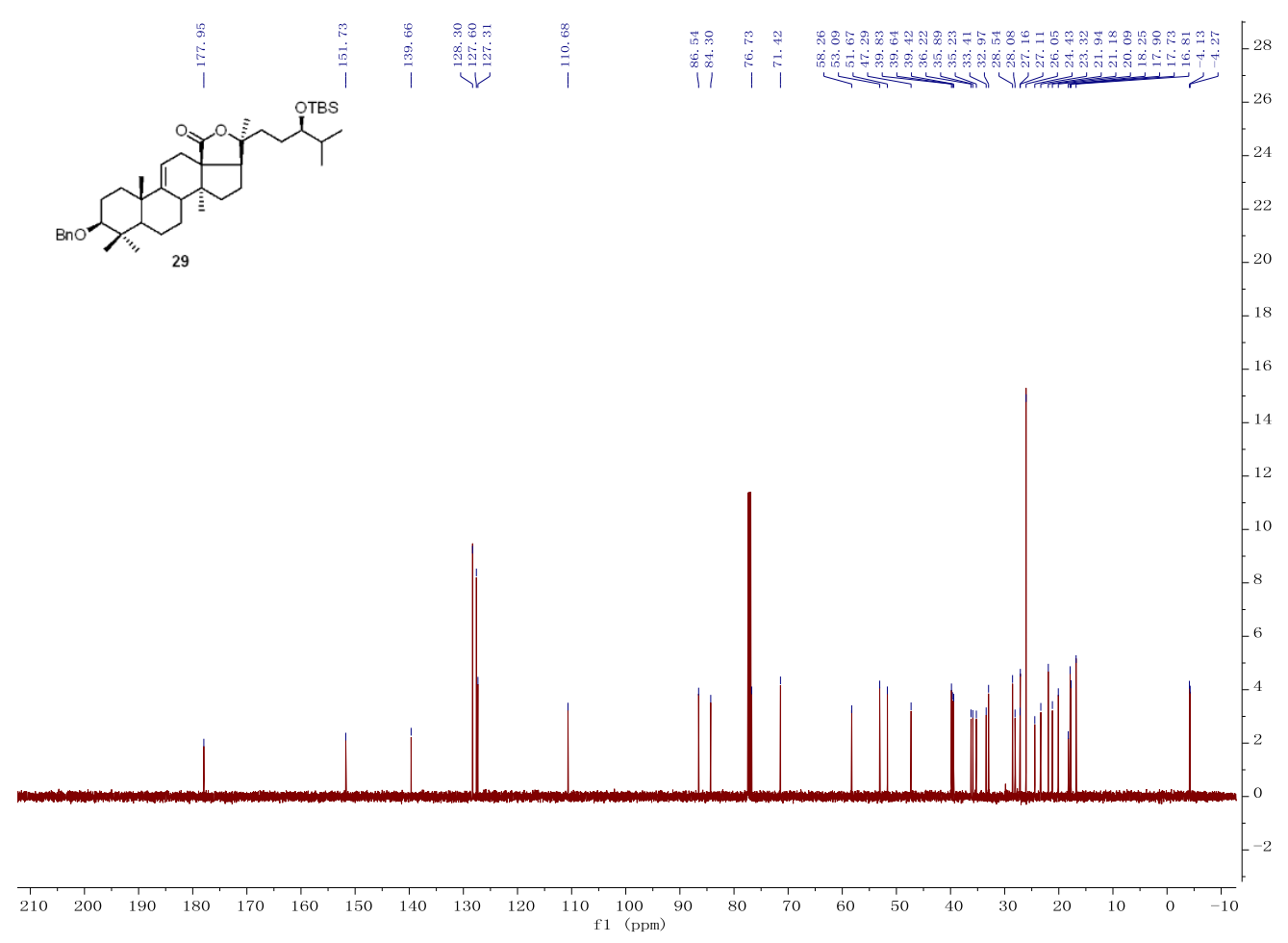

Figure S46. ${ }^{13} \mathrm{C}$ NMR spectrum of compound $29\left(125 \mathrm{MHz}, \mathrm{CDCl}_{3}\right)$ 


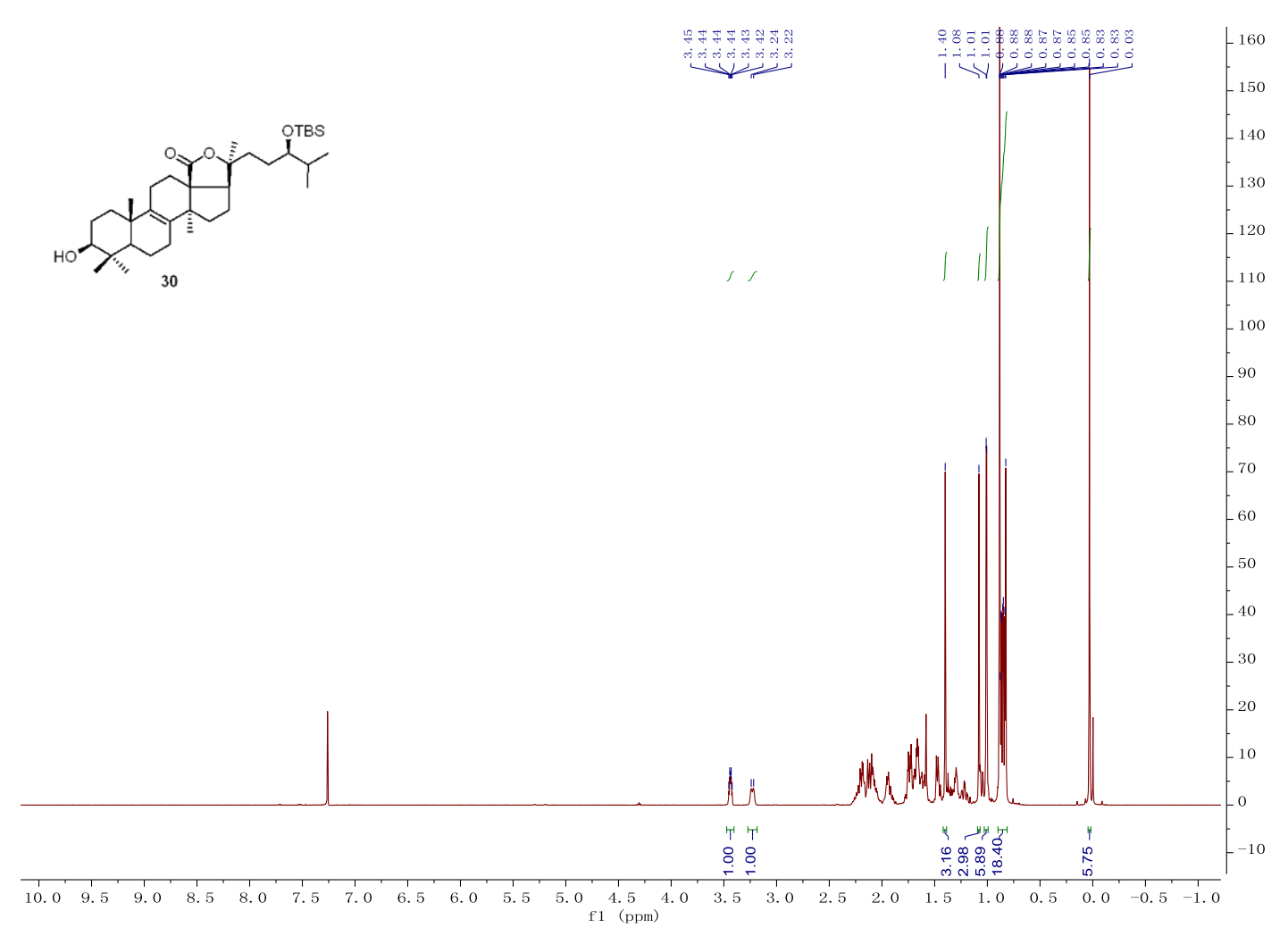

Figure S47. ${ }^{1} \mathrm{H}$ NMR spectrum of compound $30\left(500 \mathrm{MHz}, \mathrm{CDCl}_{3}\right)$

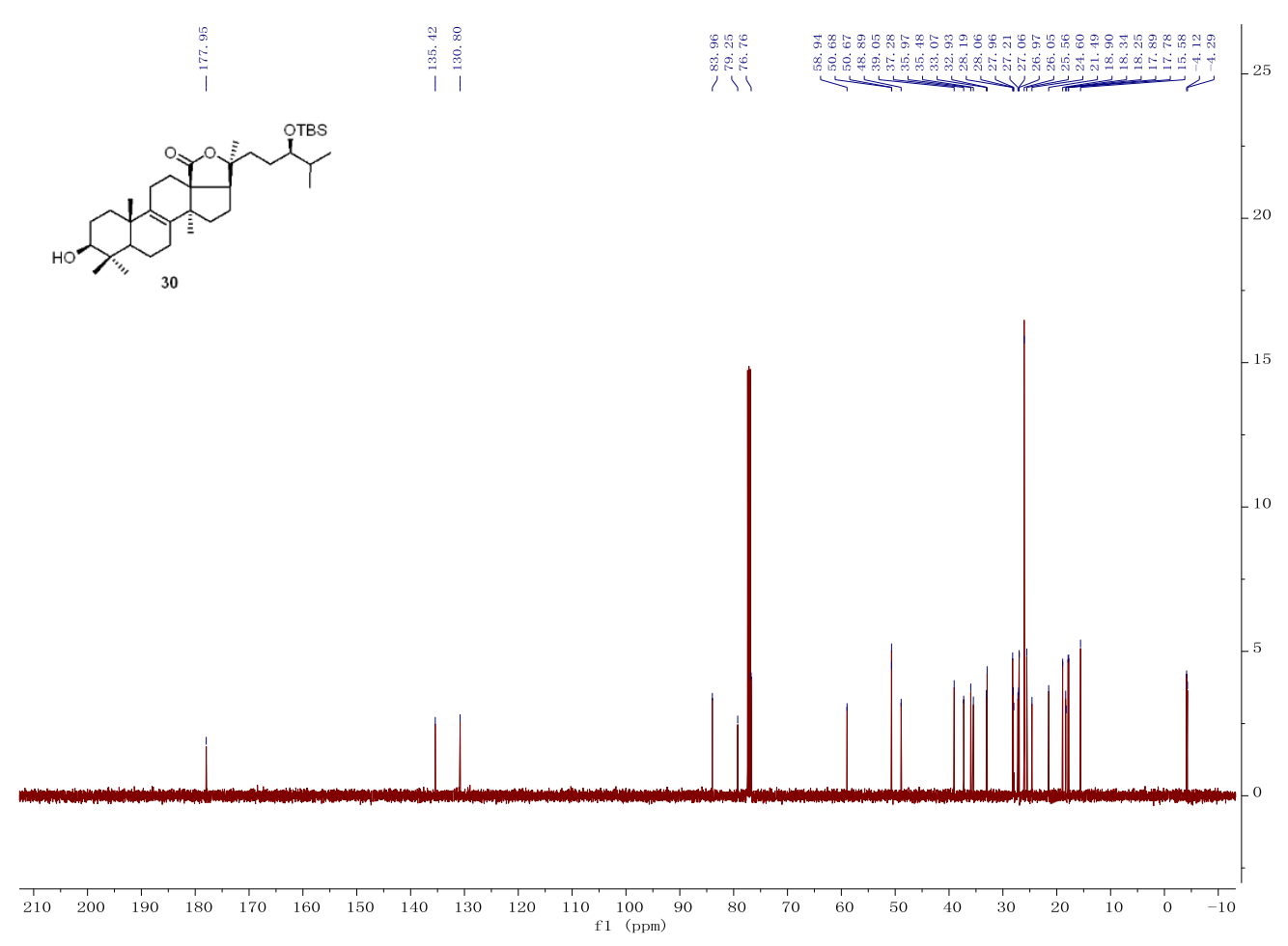

Figure S48. ${ }^{13} \mathrm{C}$ NMR spectrum of compound $30\left(125 \mathrm{MHz}, \mathrm{CDCl}_{3}\right)$ 


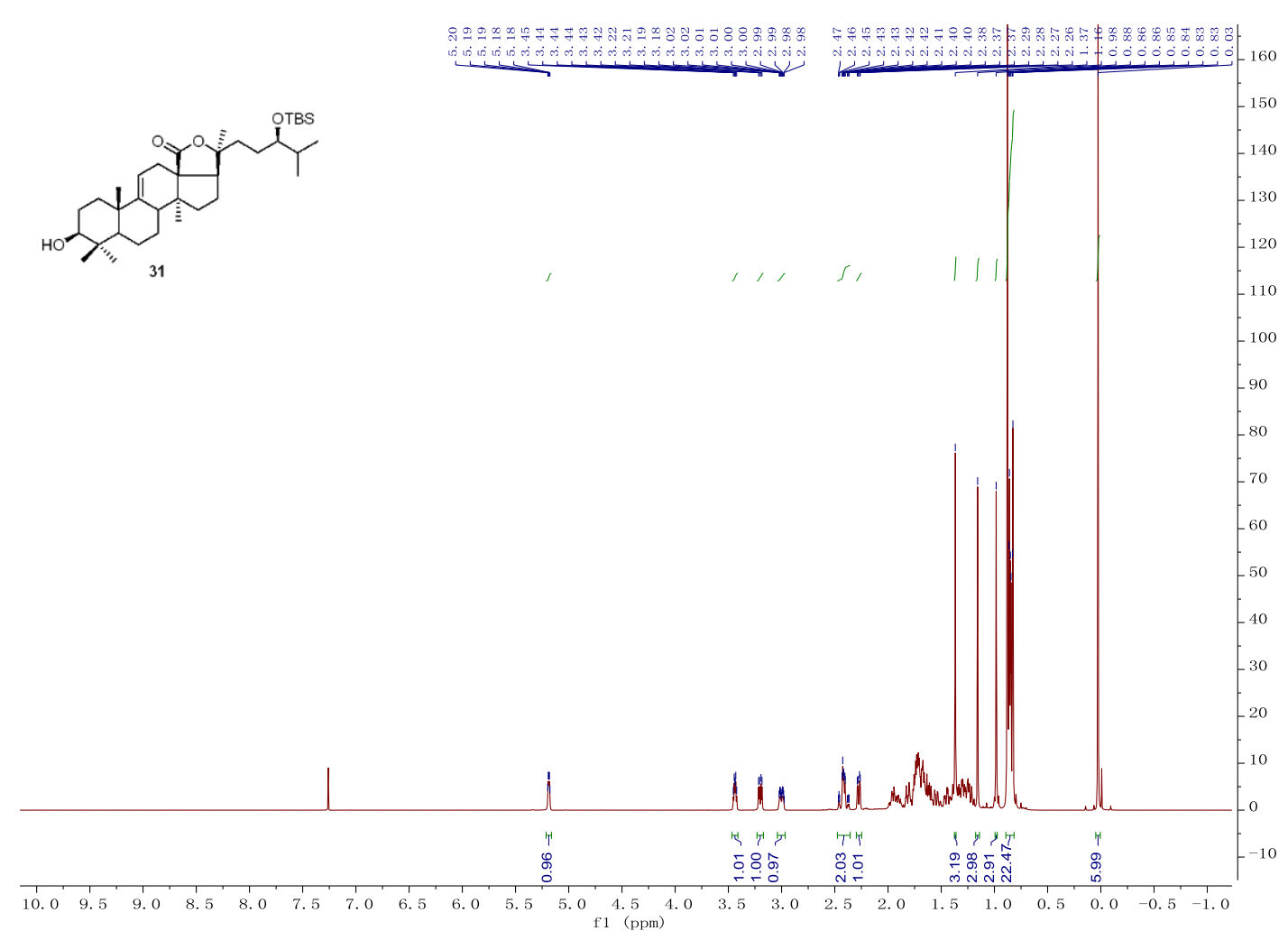

Figure S49. ${ }^{1} \mathrm{H}$ NMR spectrum of compound $31\left(500 \mathrm{MHz}, \mathrm{CDCl}_{3}\right)$
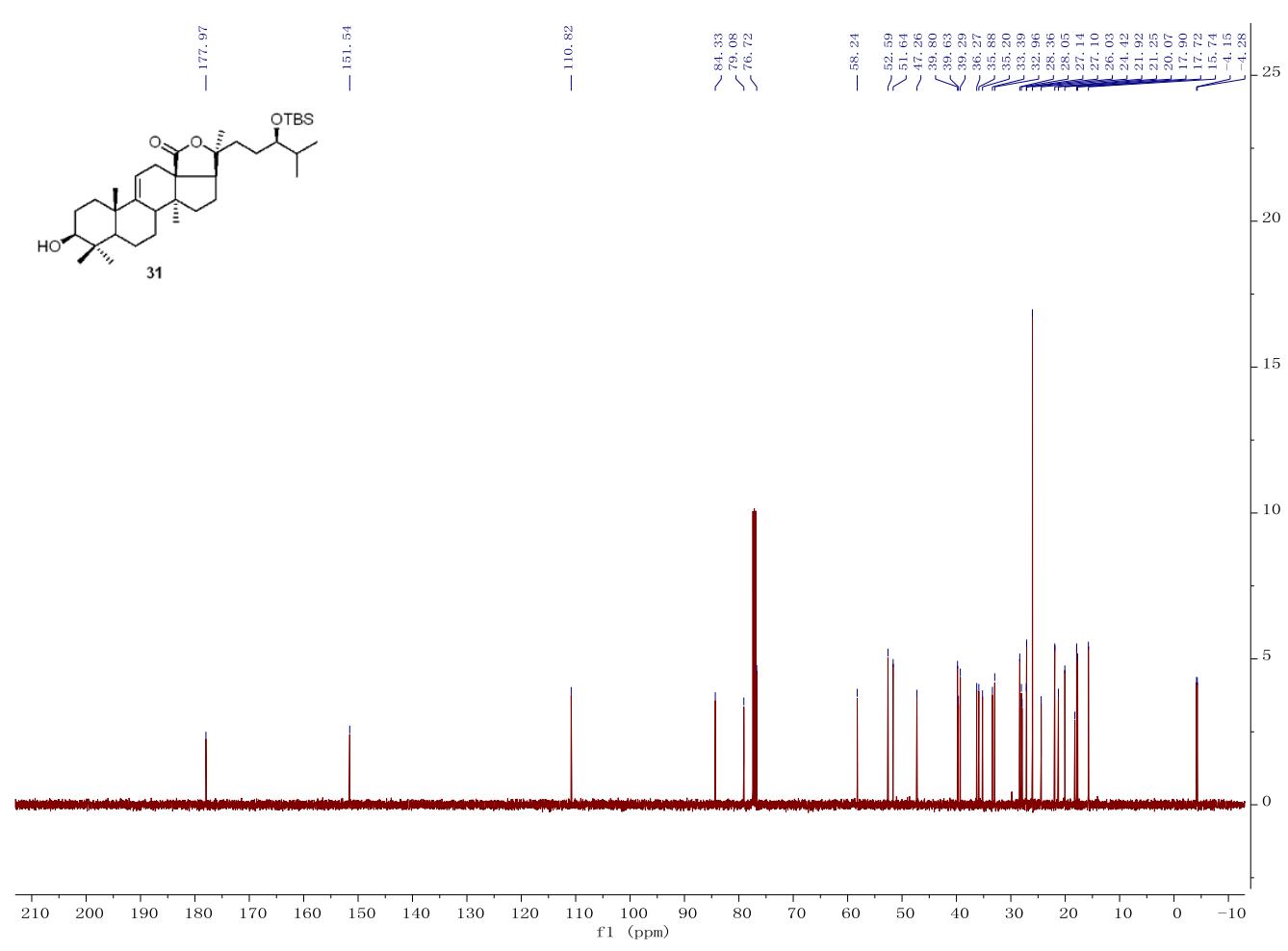

Figure S50. ${ }^{13} \mathrm{C}$ NMR spectrum of compound $31\left(125 \mathrm{MHz}, \mathrm{CDCl}_{3}\right)$ 


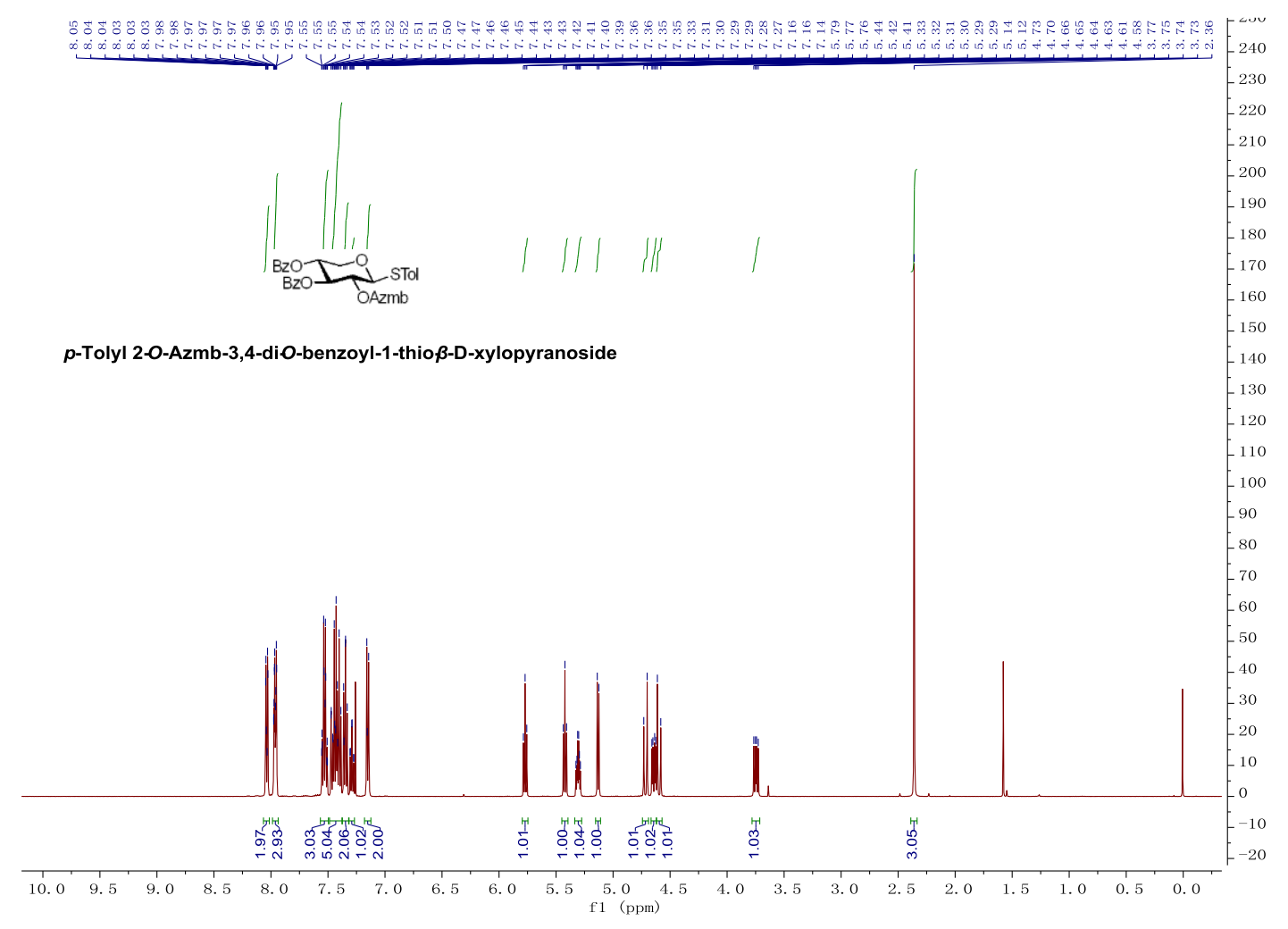

Figure S51. ${ }^{1} \mathrm{H}$ NMR spectrum of $p$-tolyl 2-O-Azmb-3,4-di- $O$-benzoyl-1-thio- $\beta$-D-

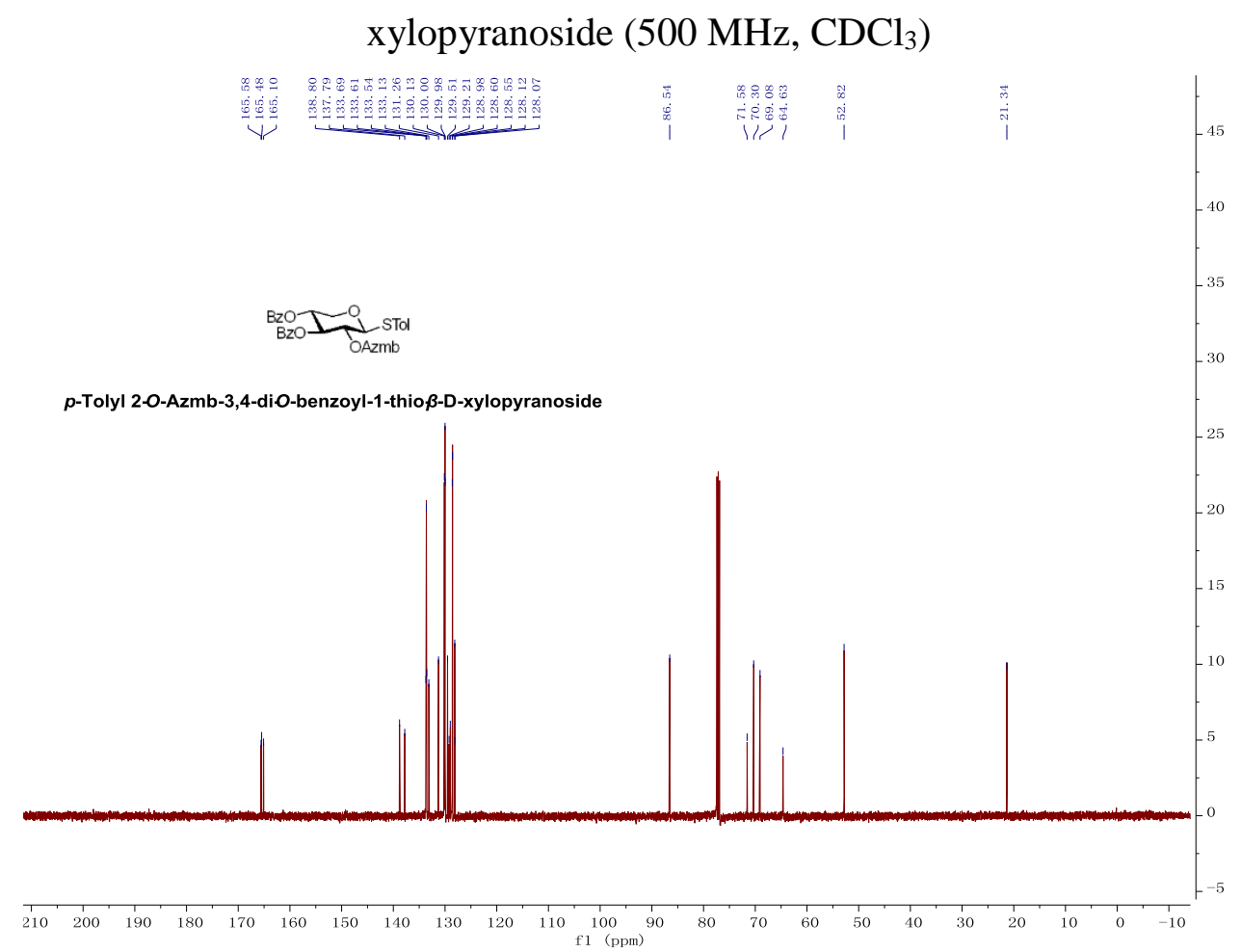

Figure S52. ${ }^{13} \mathrm{C}$ NMR spectrum of $p$-tolyl 2- $O$-Azmb-3,4-di- $O$-benzoyl-1-thio- $\beta$-Dxylopyranoside $\left(125 \mathrm{MHz}, \mathrm{CDCl}_{3}\right)$ 


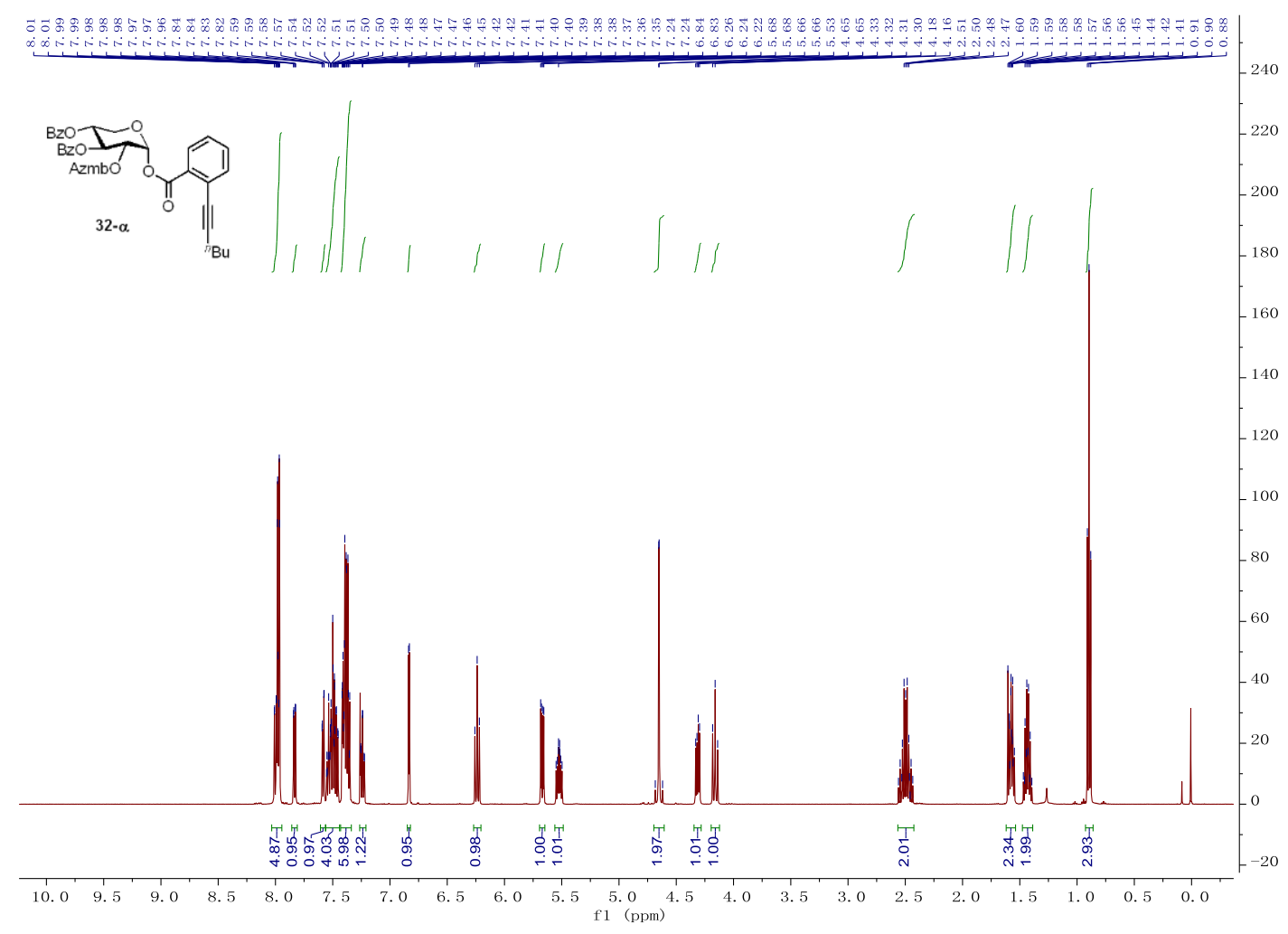

Figure S53. ${ }^{1} \mathrm{H}$ NMR spectrum of compound $\mathbf{3 2} \boldsymbol{\alpha}\left(500 \mathrm{MHz}, \mathrm{CDCl}_{3}\right)$

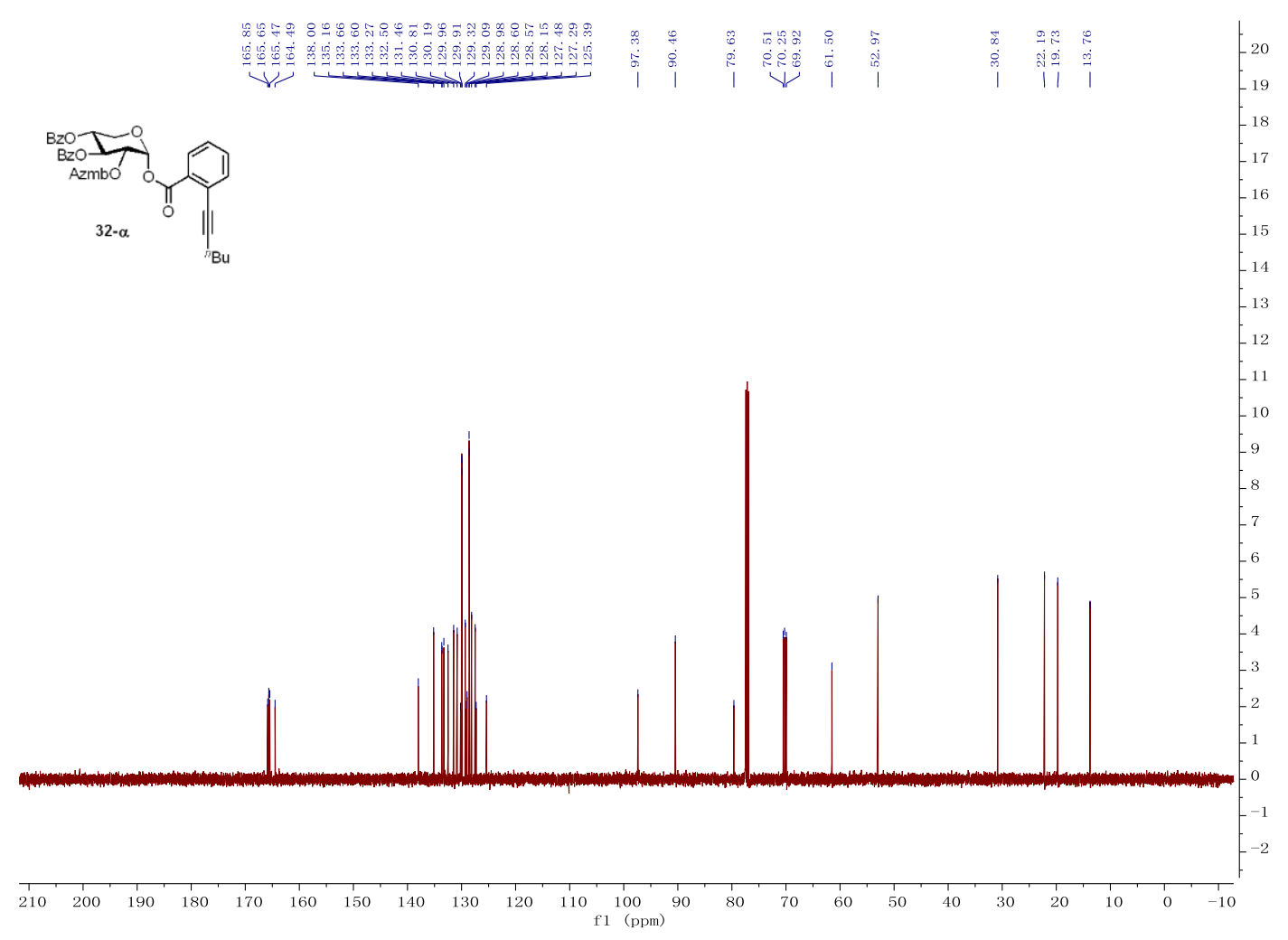

Figure S54. ${ }^{13} \mathrm{C}$ NMR spectrum of compound $\mathbf{3 2} \alpha\left(125 \mathrm{MHz}, \mathrm{CDCl}_{3}\right)$ 


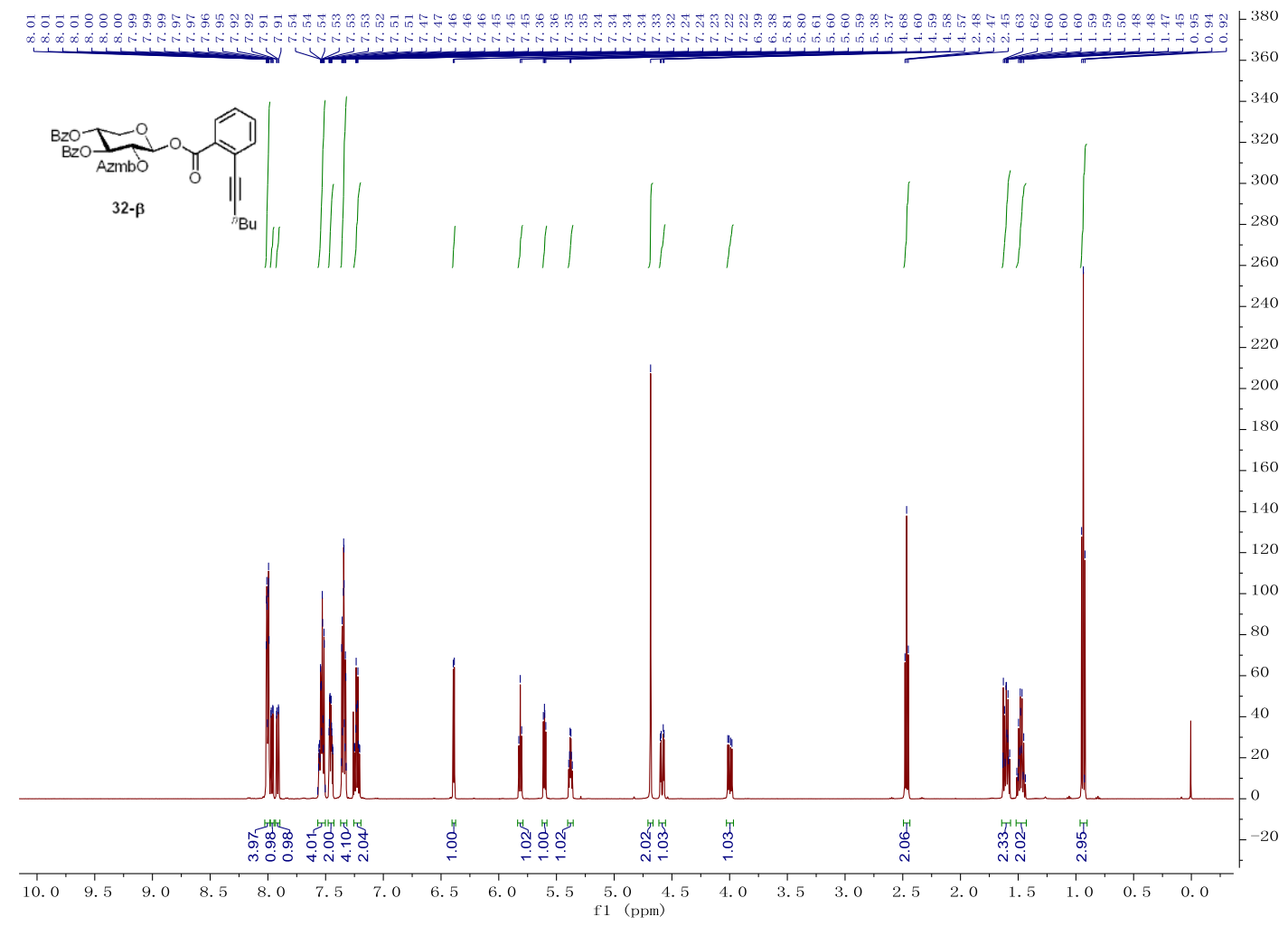

Figure S55. ${ }^{1} \mathrm{H}$ NMR spectrum of compound $\mathbf{3 2 \beta}\left(500 \mathrm{MHz}, \mathrm{CDCl}_{3}\right)$

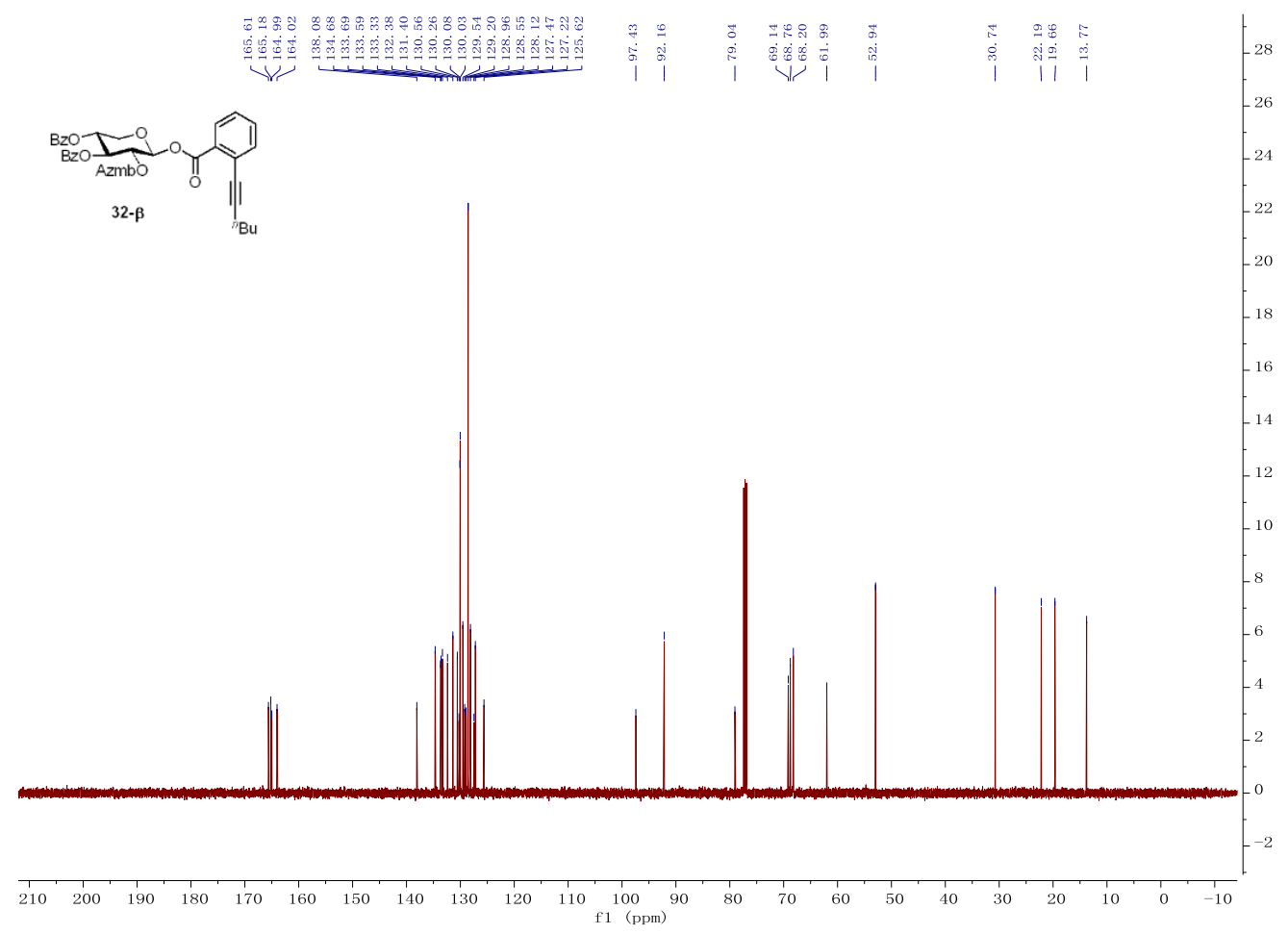

Figure S56. ${ }^{13} \mathrm{C}$ NMR spectrum of compound $32 \beta\left(125 \mathrm{MHz}, \mathrm{CDCl}_{3}\right)$ 


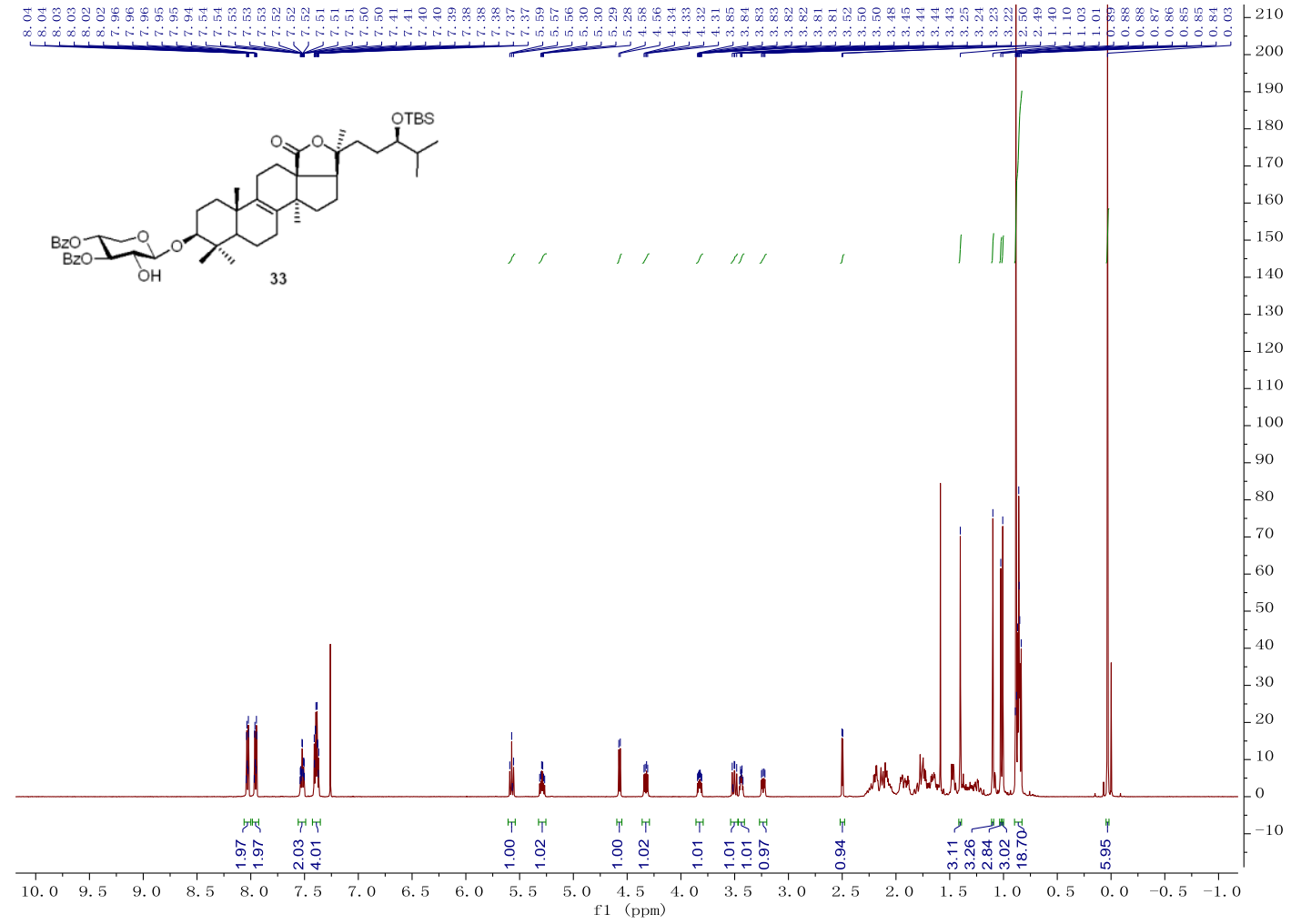

Figure S57. ${ }^{1} \mathrm{H}$ NMR spectrum of compound $33\left(500 \mathrm{MHz}, \mathrm{CDCl}_{3}\right)$

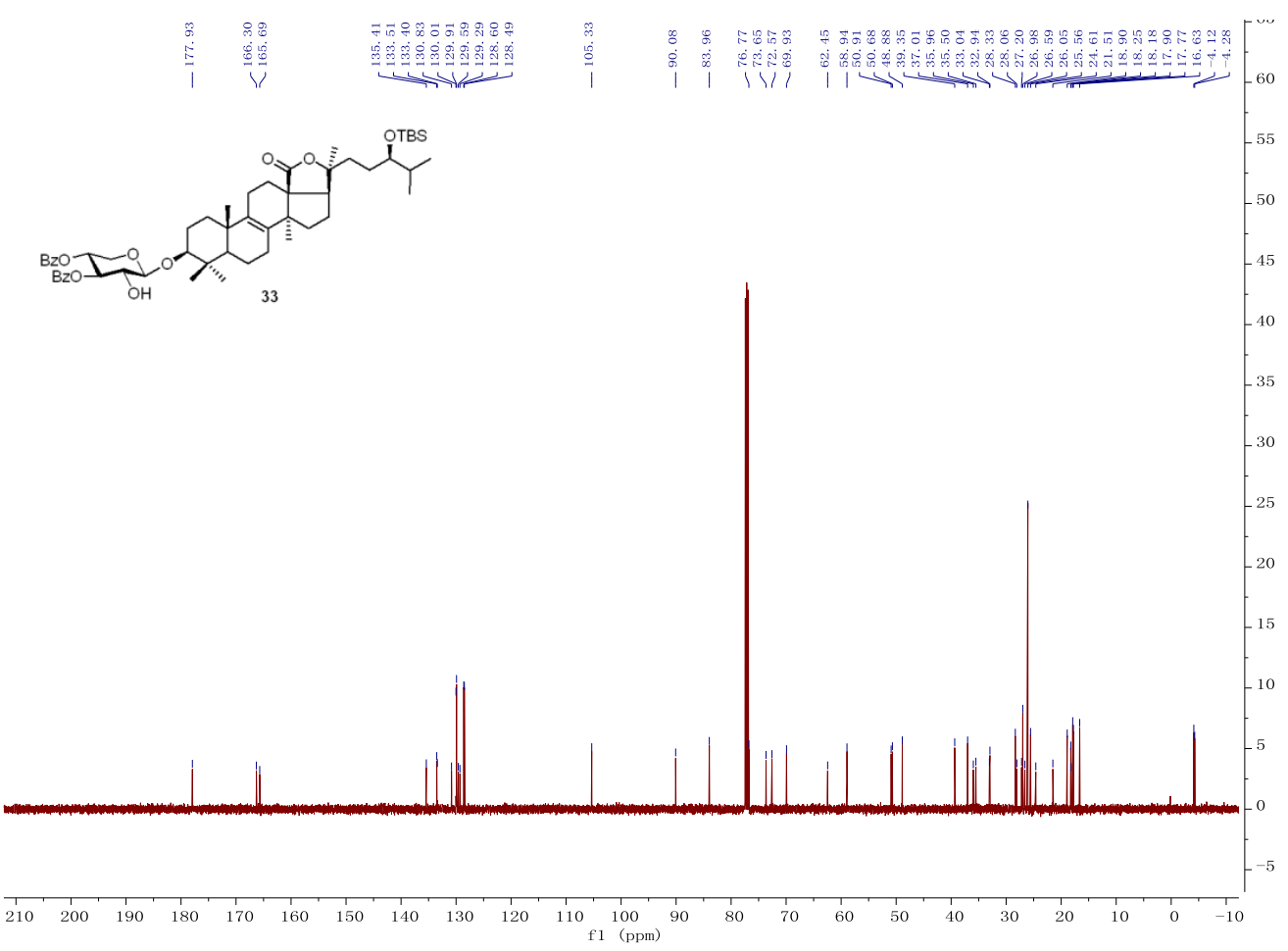

Figure S58. ${ }^{13} \mathrm{C}$ NMR spectrum of compound $33\left(125 \mathrm{MHz}, \mathrm{CDCl}_{3}\right)$ 


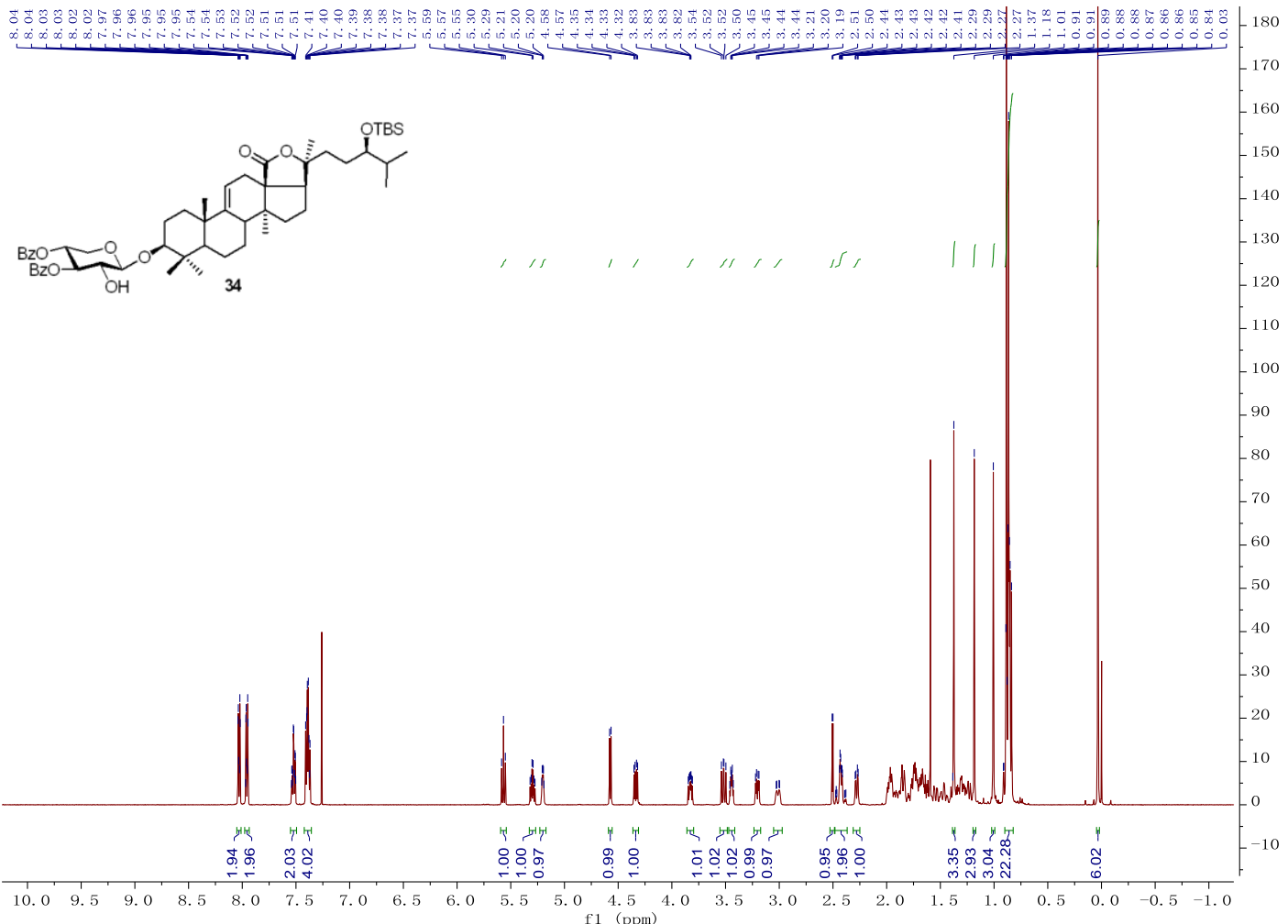

Figure S59. ${ }^{1} \mathrm{H}$ NMR spectrum of compound $34\left(500 \mathrm{MHz}, \mathrm{CDCl}_{3}\right)$

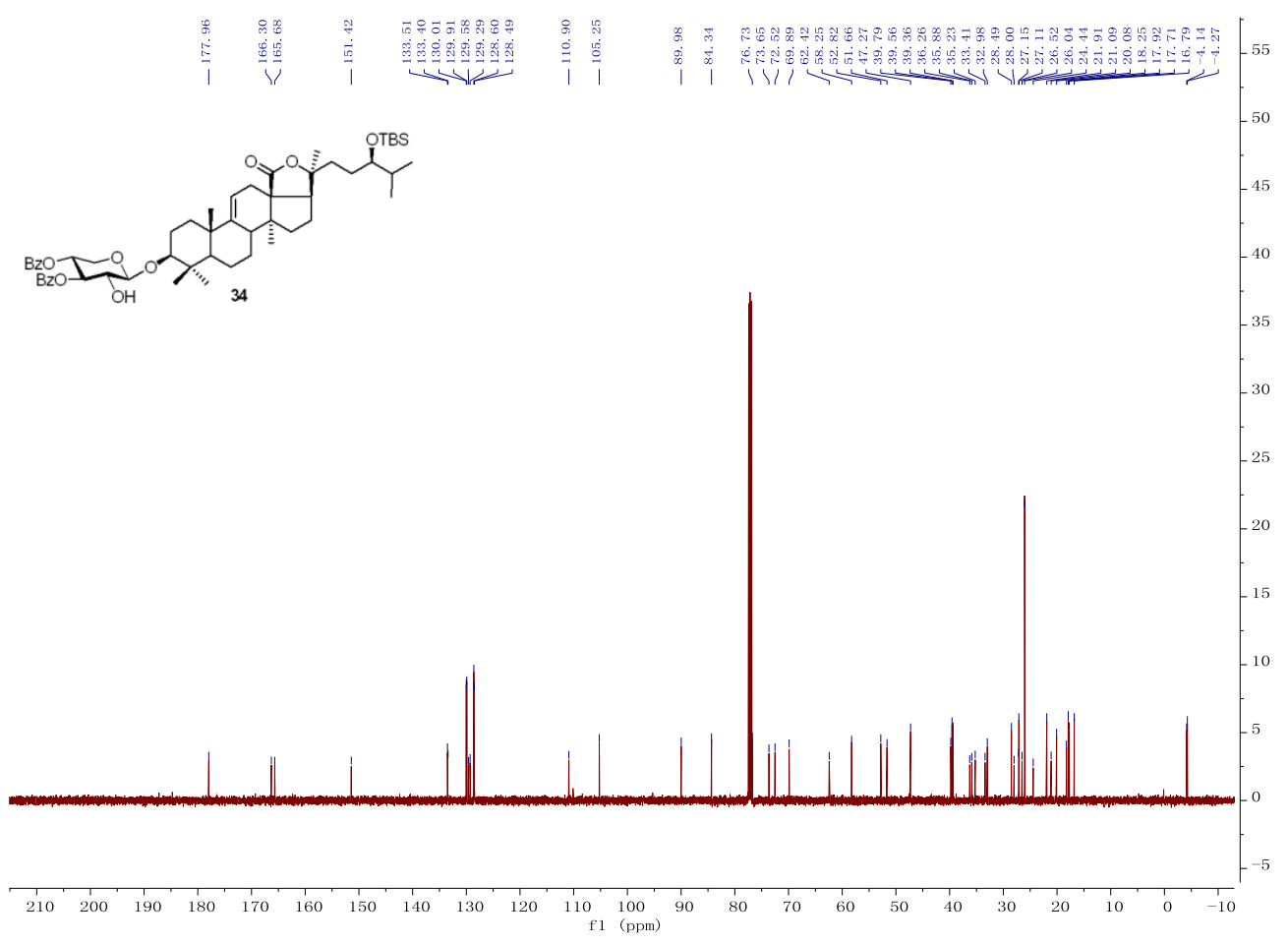

Figure S60. ${ }^{13} \mathrm{C}$ NMR spectrum of compound $34\left(125 \mathrm{MHz}, \mathrm{CDCl}_{3}\right)$ 


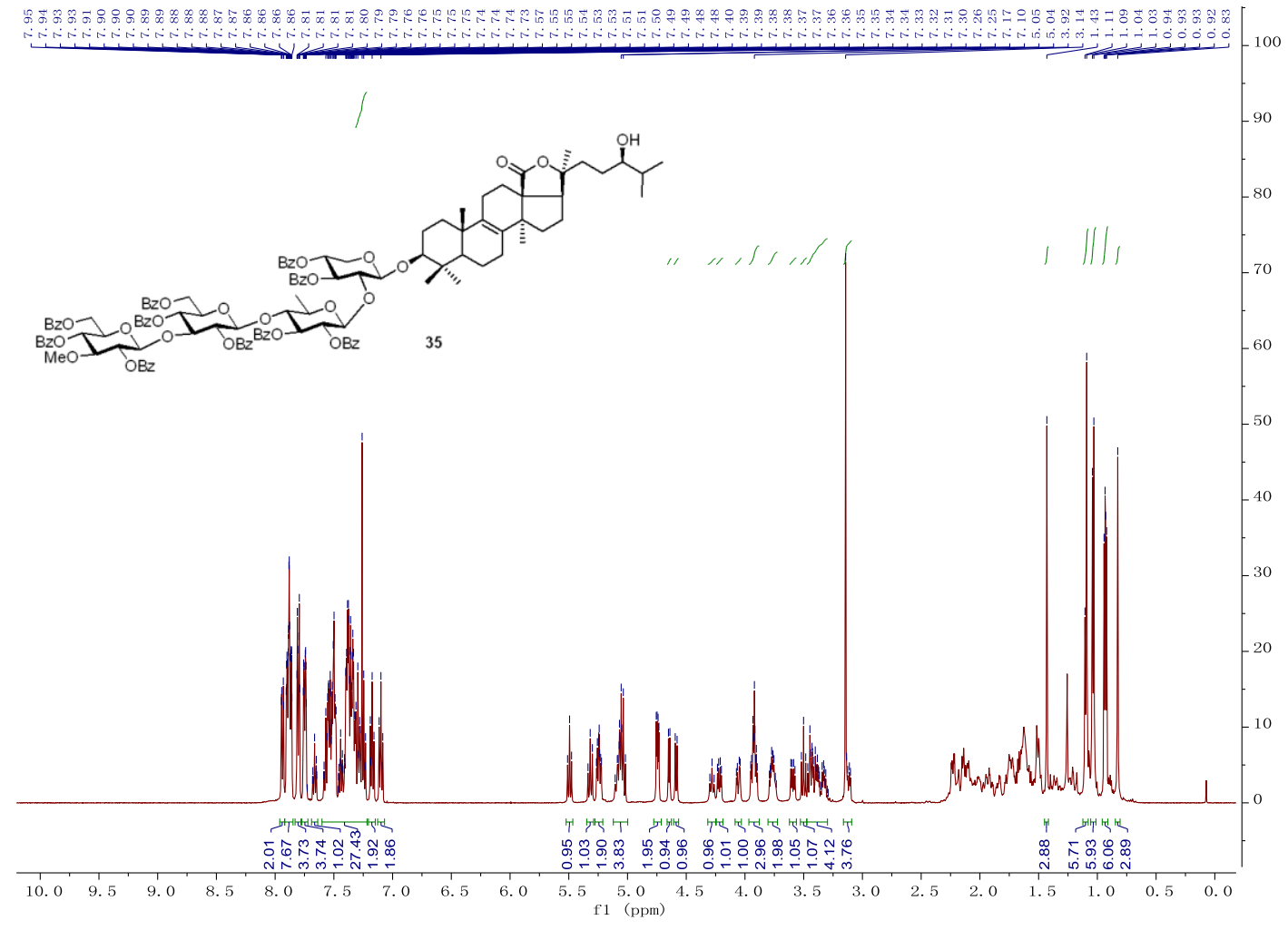

Figure S61. ${ }^{1} \mathrm{H}$ NMR spectrum of compound $35\left(500 \mathrm{MHz}, \mathrm{CDCl}_{3}\right)$

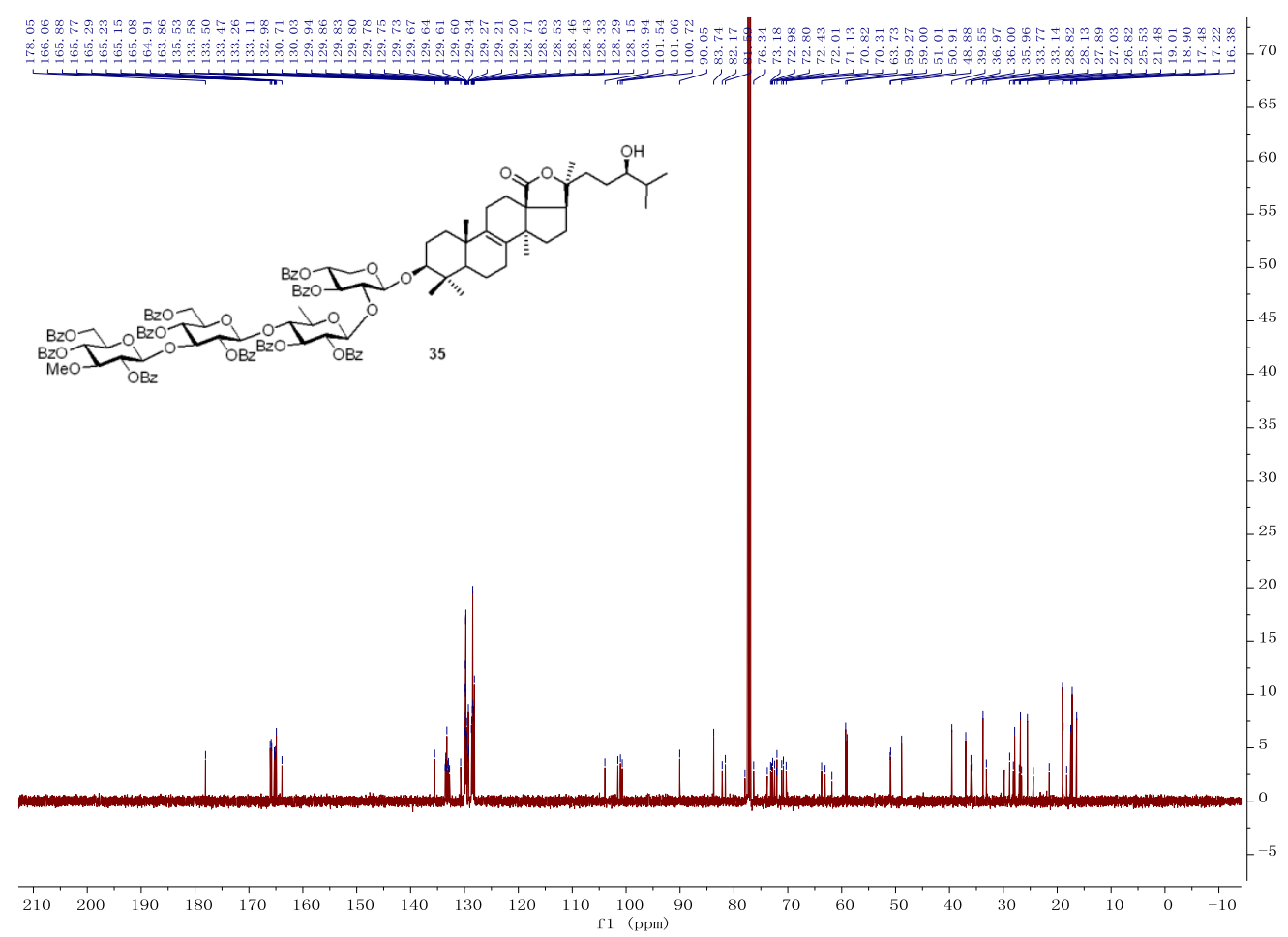

Figure S62. ${ }^{13} \mathrm{C}$ NMR spectrum of compound $35\left(125 \mathrm{MHz}, \mathrm{CDCl}_{3}\right)$ 


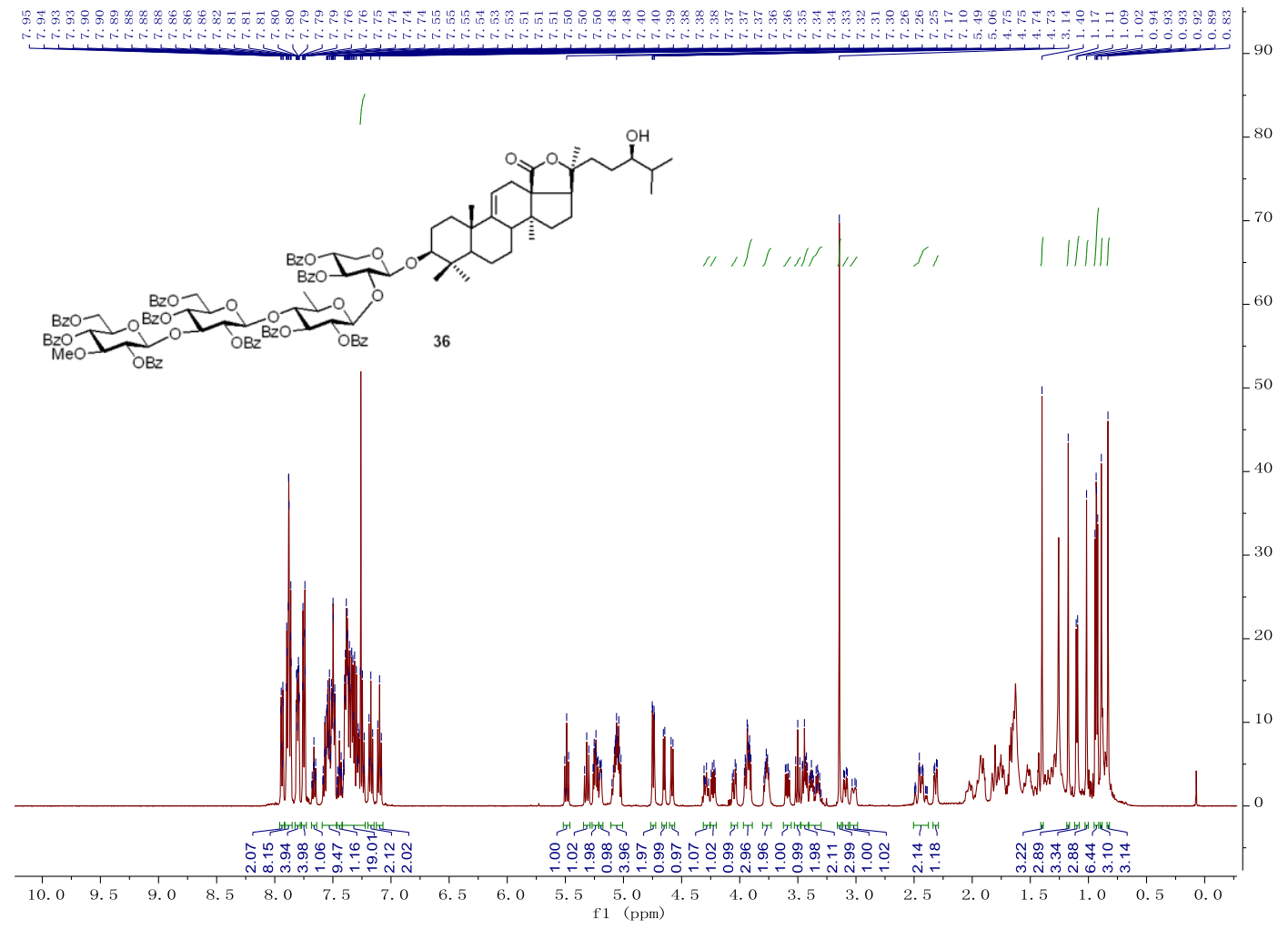

Figure S63. ${ }^{1} \mathrm{H}$ NMR spectrum of compound $36\left(500 \mathrm{MHz}, \mathrm{CDCl}_{3}\right)$

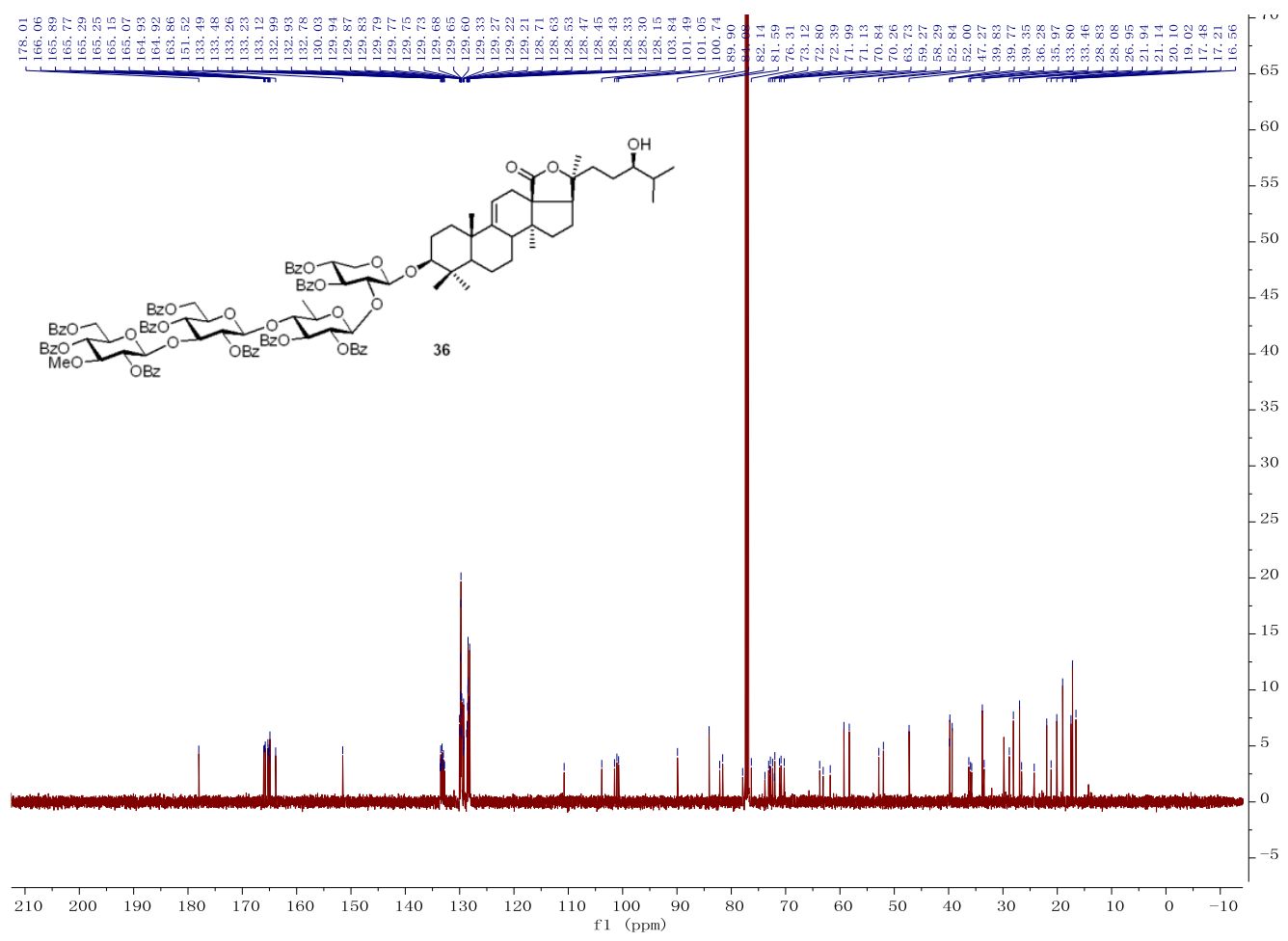

Figure S64. ${ }^{13} \mathrm{C}$ NMR spectrum of compound $36\left(125 \mathrm{MHz}, \mathrm{CDCl}_{3}\right)$ 


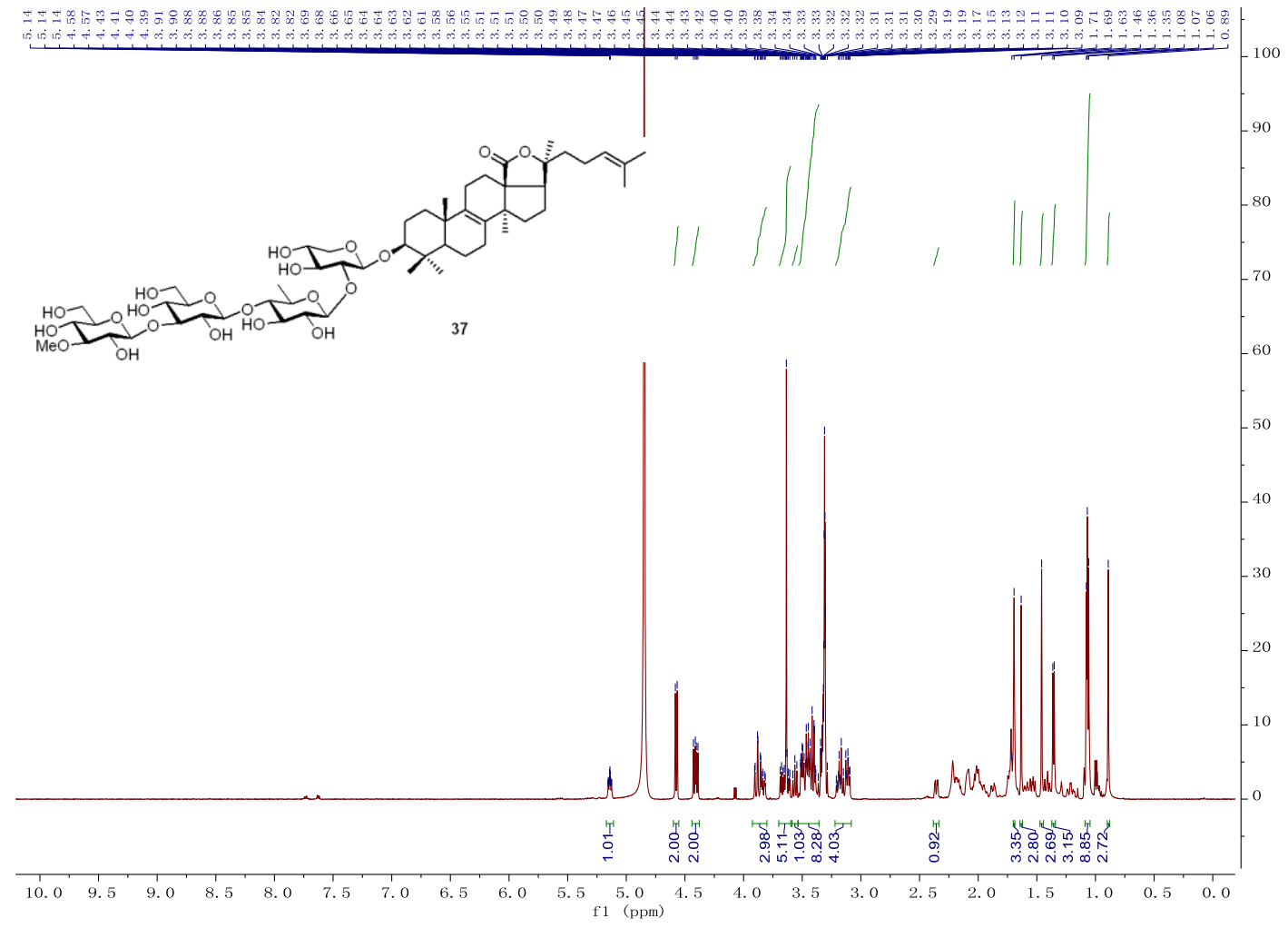

Figure S65. ${ }^{1} \mathrm{H}$ NMR spectrum of compound 37 (500 MHz, CD $\left.{ }_{3} \mathrm{OD}\right)$

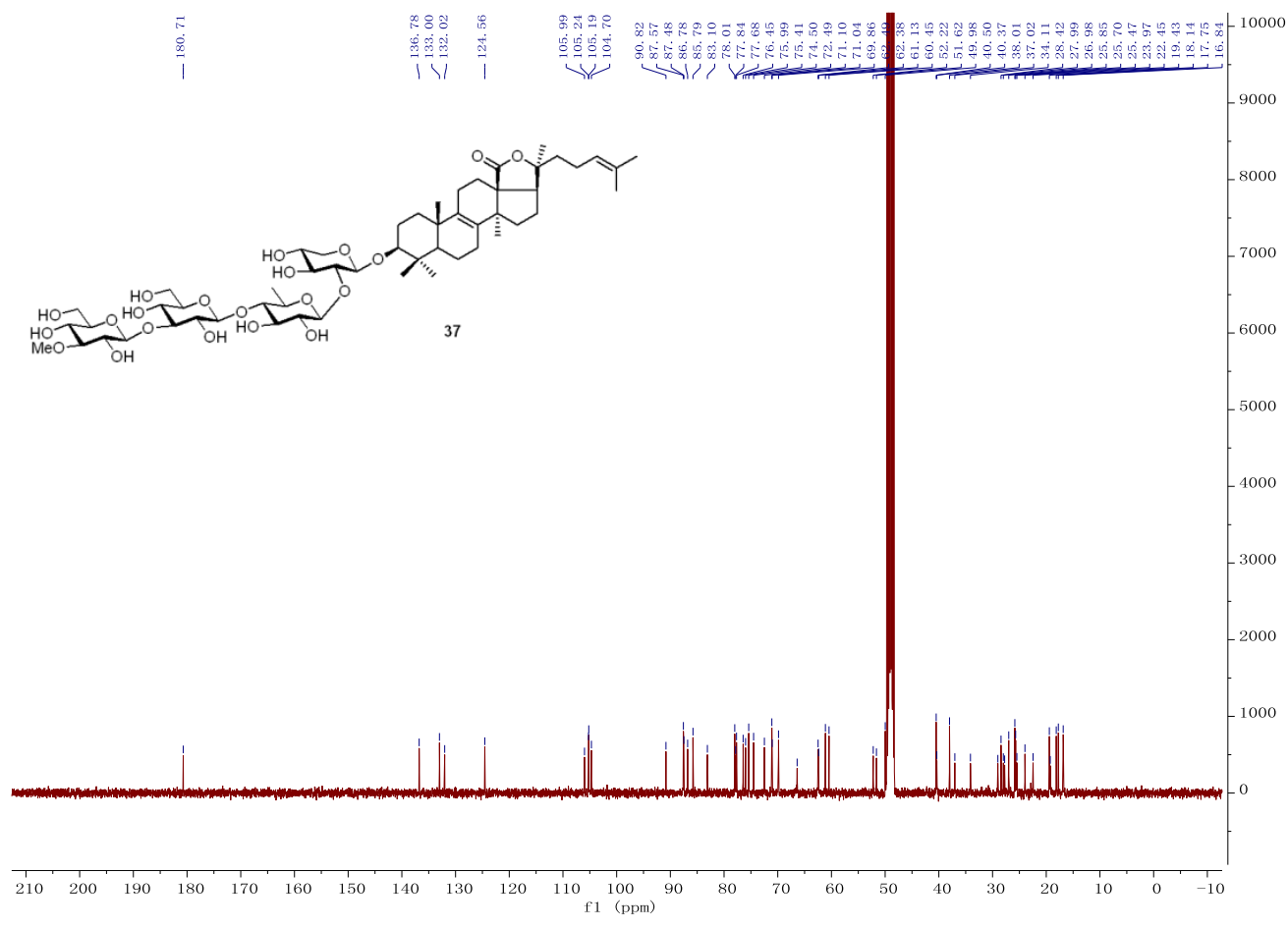

Figure S66. ${ }^{13} \mathrm{C}$ NMR spectrum of compound $37\left(100 \mathrm{MHz}, \mathrm{CD}_{3} \mathrm{OD}\right)$ 


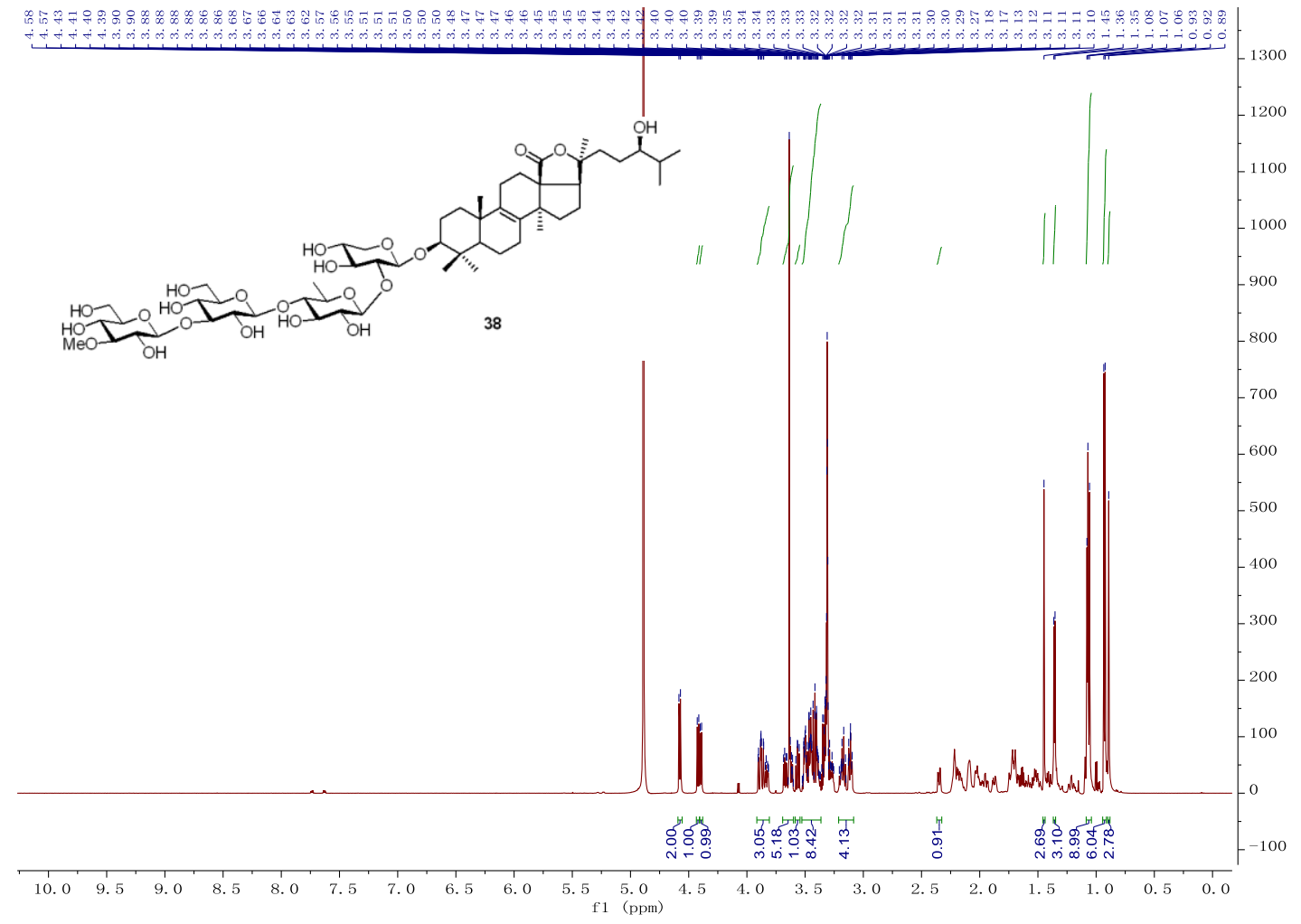

Figure S67. ${ }^{1} \mathrm{H}$ NMR spectrum of compound $38\left(500 \mathrm{MHz}, \mathrm{CD}_{3} \mathrm{OD}\right)$

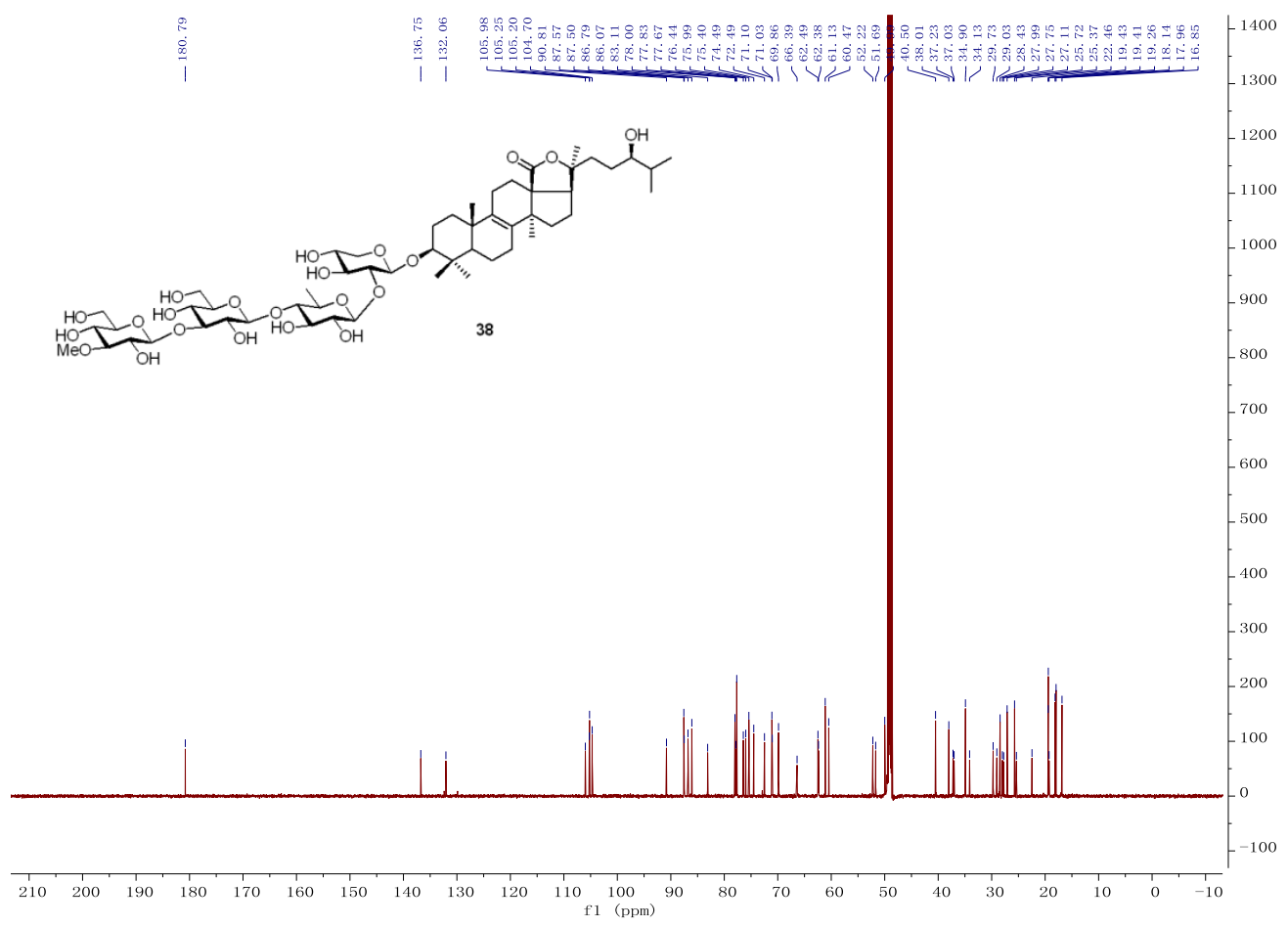

Figure S68. ${ }^{13} \mathrm{C}$ NMR spectrum of compound $38\left(125 \mathrm{MHz}, \mathrm{CD}_{3} \mathrm{OD}\right)$ 


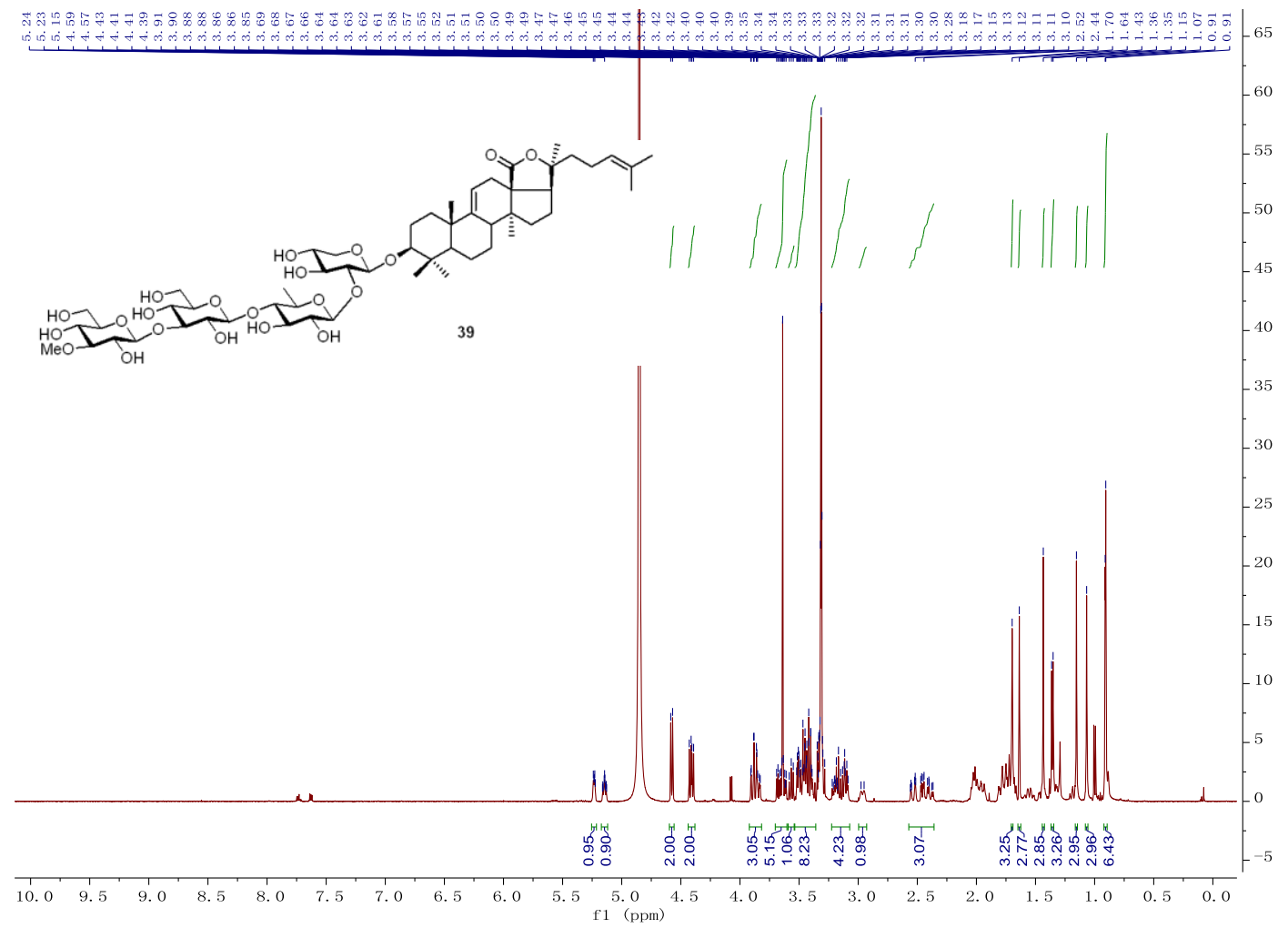

Figure S69. ${ }^{1} \mathrm{H}$ NMR spectrum of compound 39 (500 MHz, $\left.\mathrm{CD}_{3} \mathrm{OD}\right)$

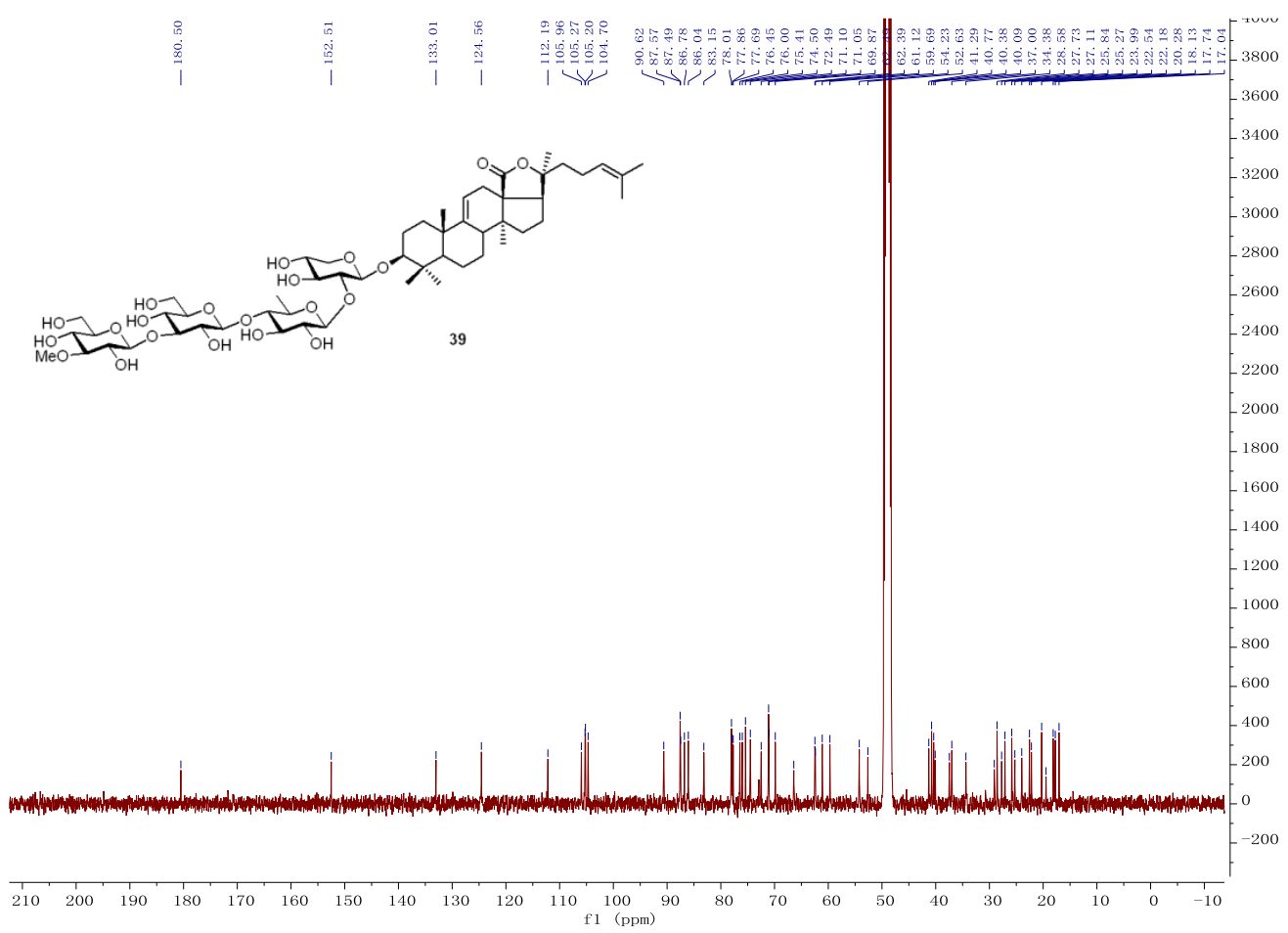

Figure S70. ${ }^{13} \mathrm{C}$ NMR spectrum of compound $39\left(100 \mathrm{MHz}, \mathrm{CD}_{3} \mathrm{OD}\right)$ 


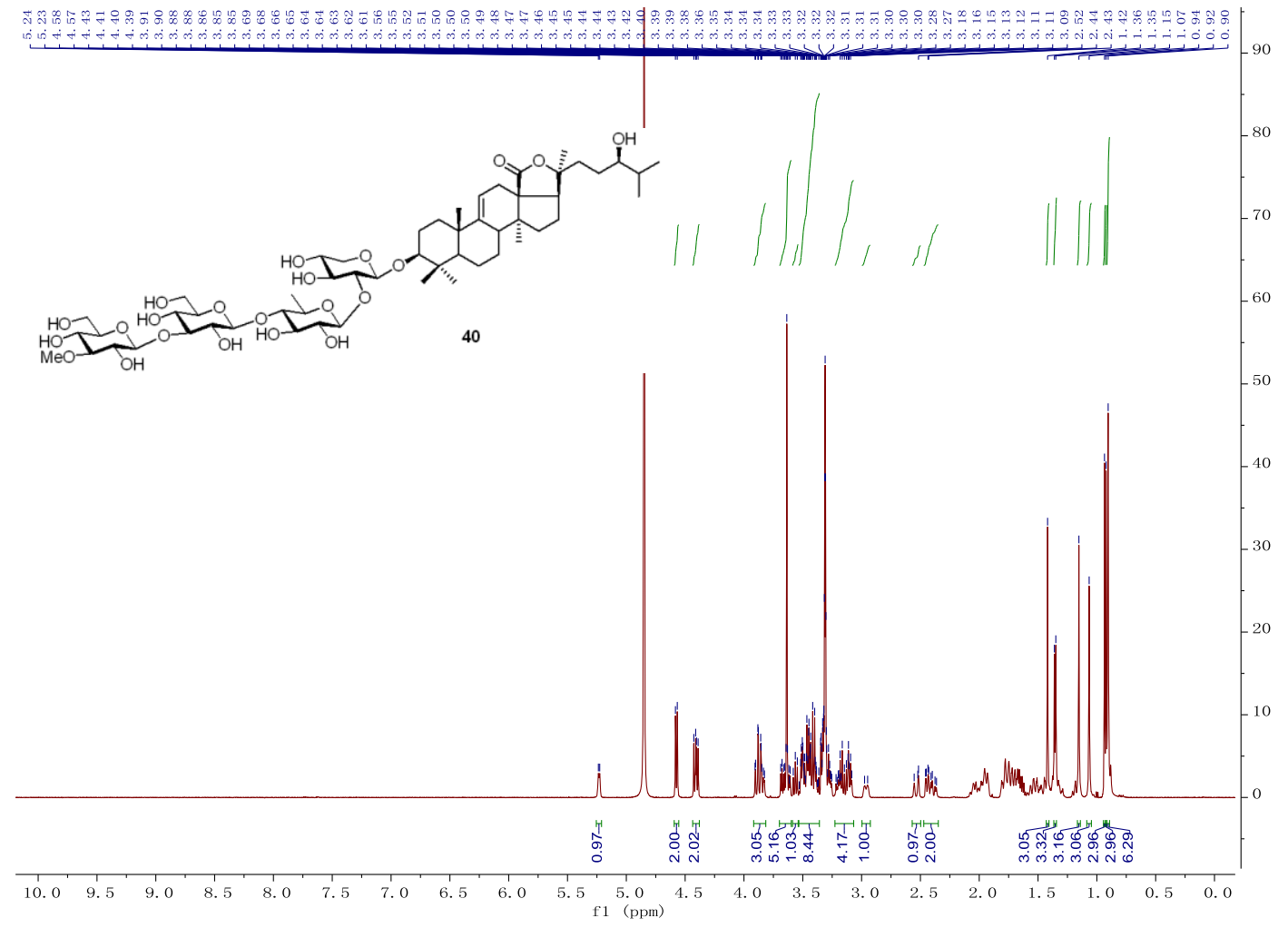

Figure S71. ${ }^{1} \mathrm{H}$ NMR spectrum of compound $40\left(500 \mathrm{MHz}, \mathrm{CD}_{3} \mathrm{OD}\right)$

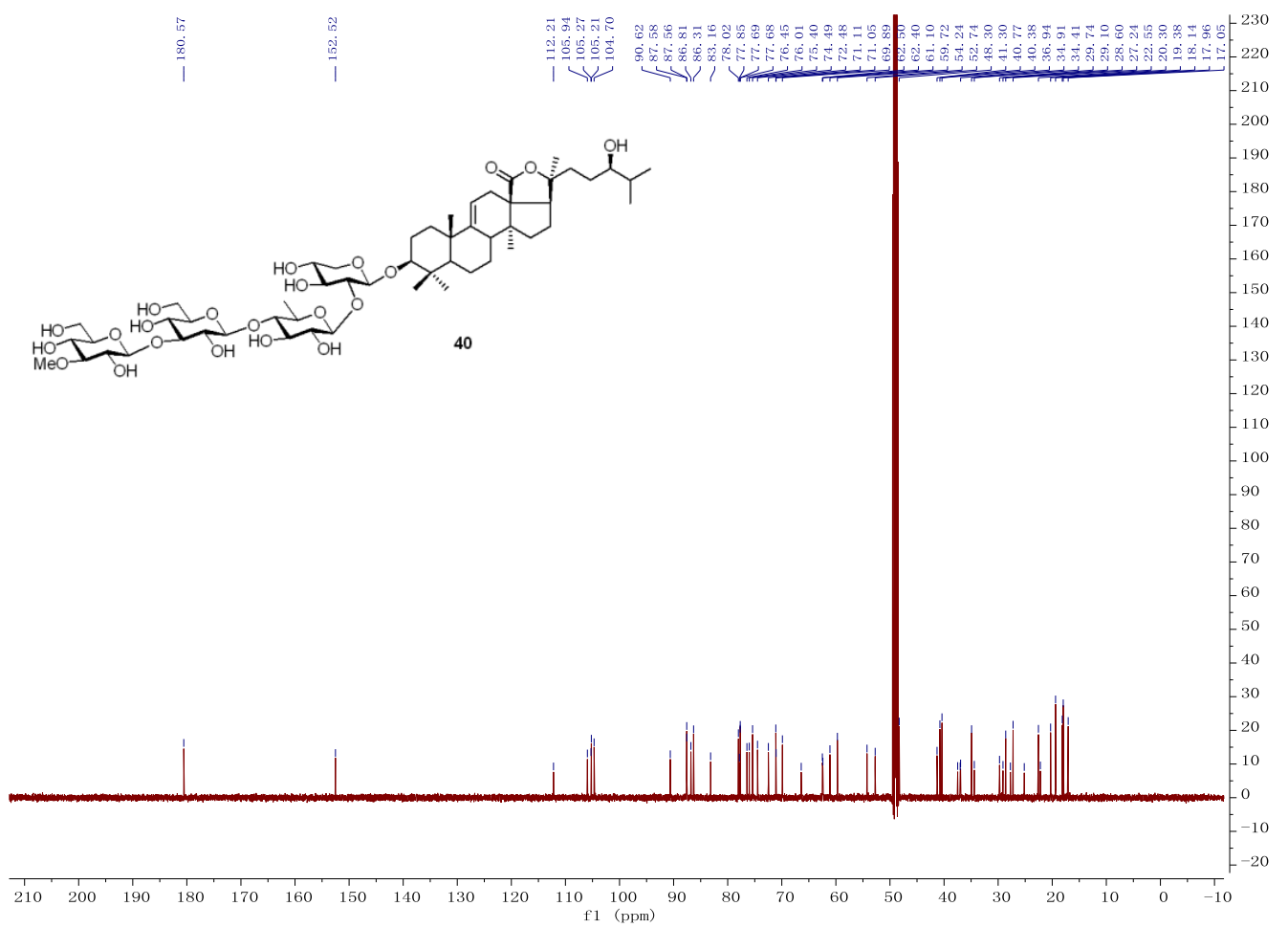

Figure S72. ${ }^{13} \mathrm{C}$ NMR spectrum of compound $40\left(125 \mathrm{MHz}, \mathrm{CD}_{3} \mathrm{OD}\right)$ 


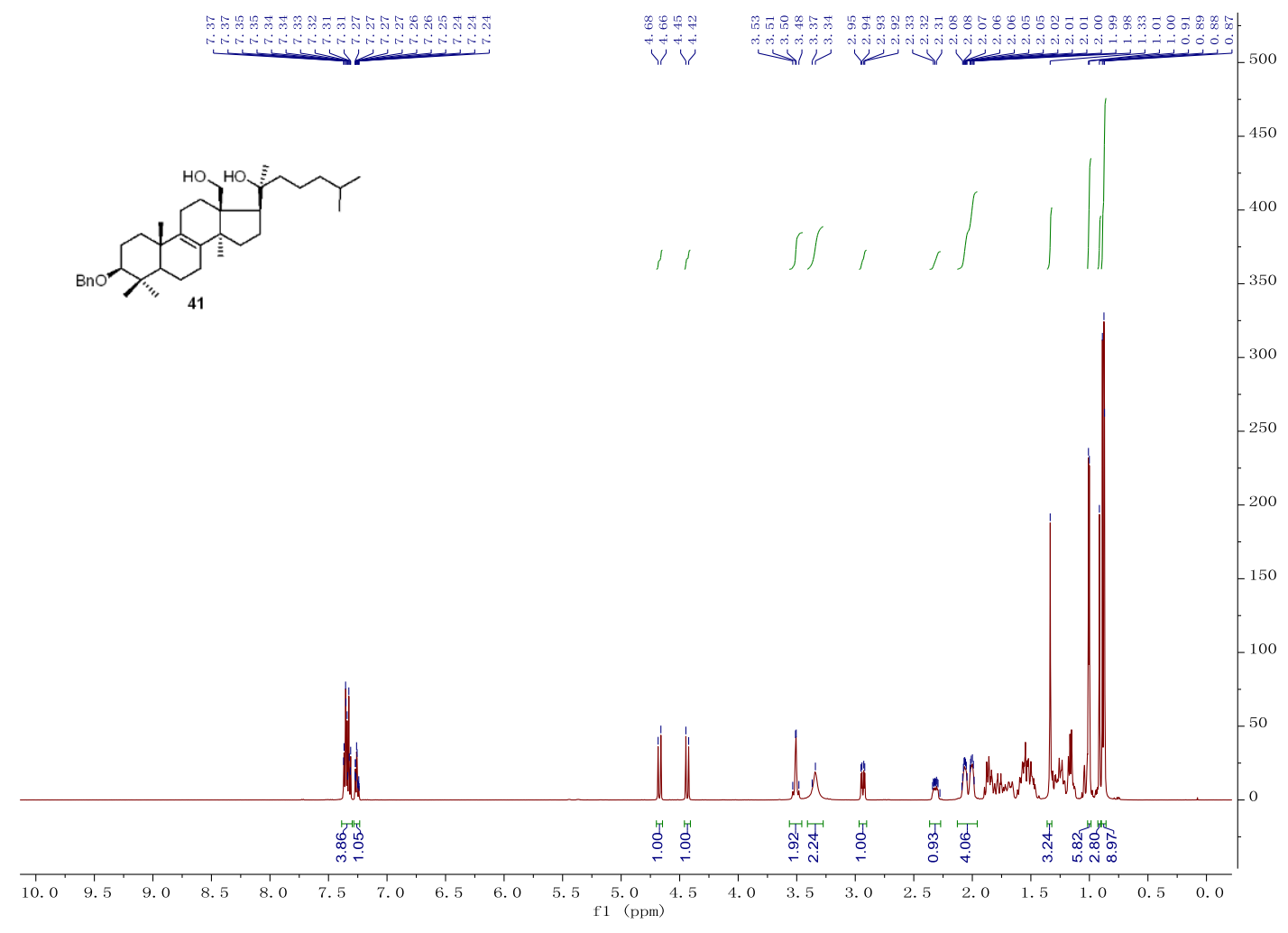

Figure S73. ${ }^{1} \mathrm{H}$ NMR spectrum of compound $41\left(500 \mathrm{MHz}, \mathrm{CDCl}_{3}\right)$

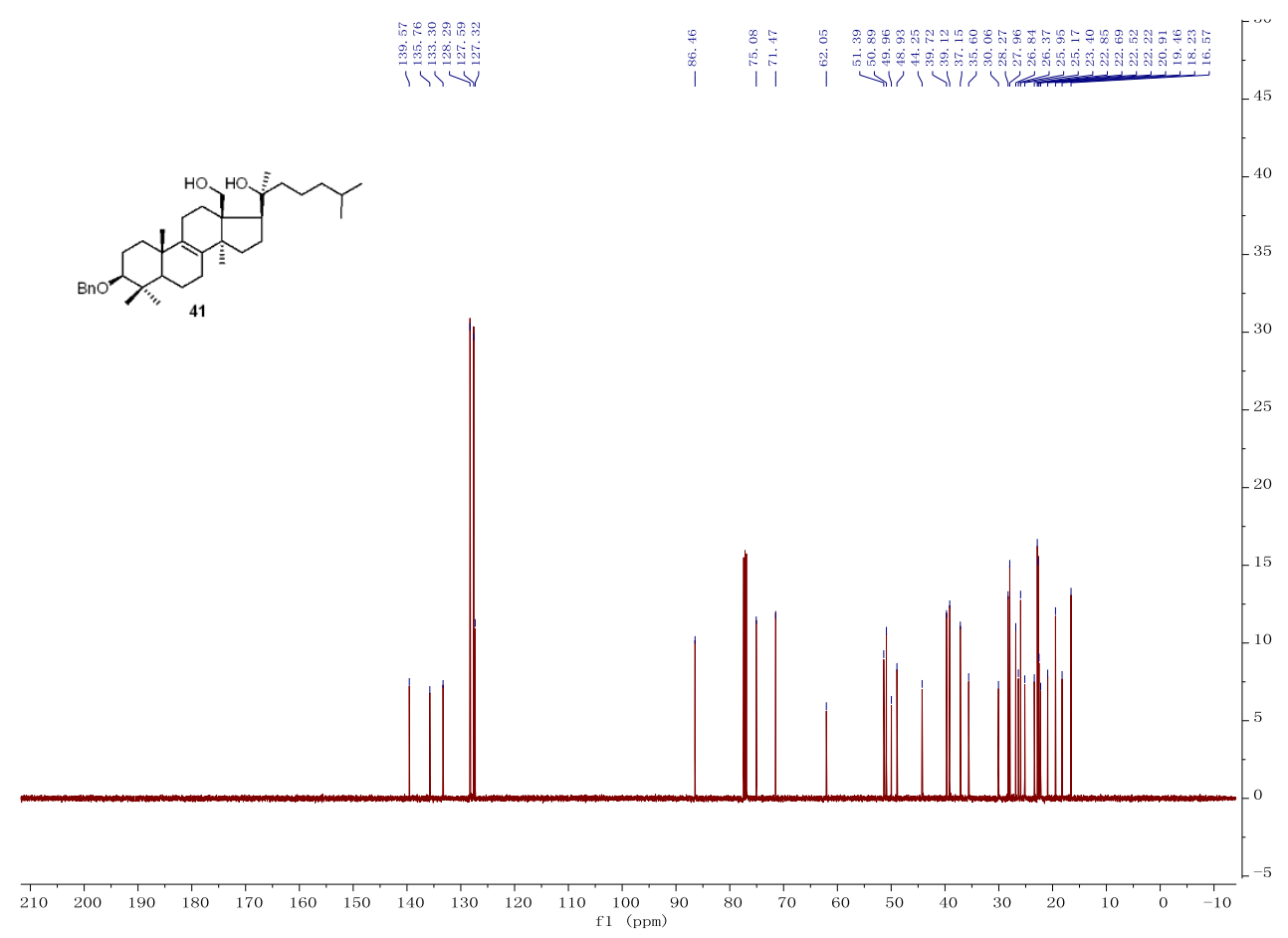

Figure S74. ${ }^{13} \mathrm{C}$ NMR spectrum of compound $41\left(125 \mathrm{MHz}, \mathrm{CDCl}_{3}\right)$ 


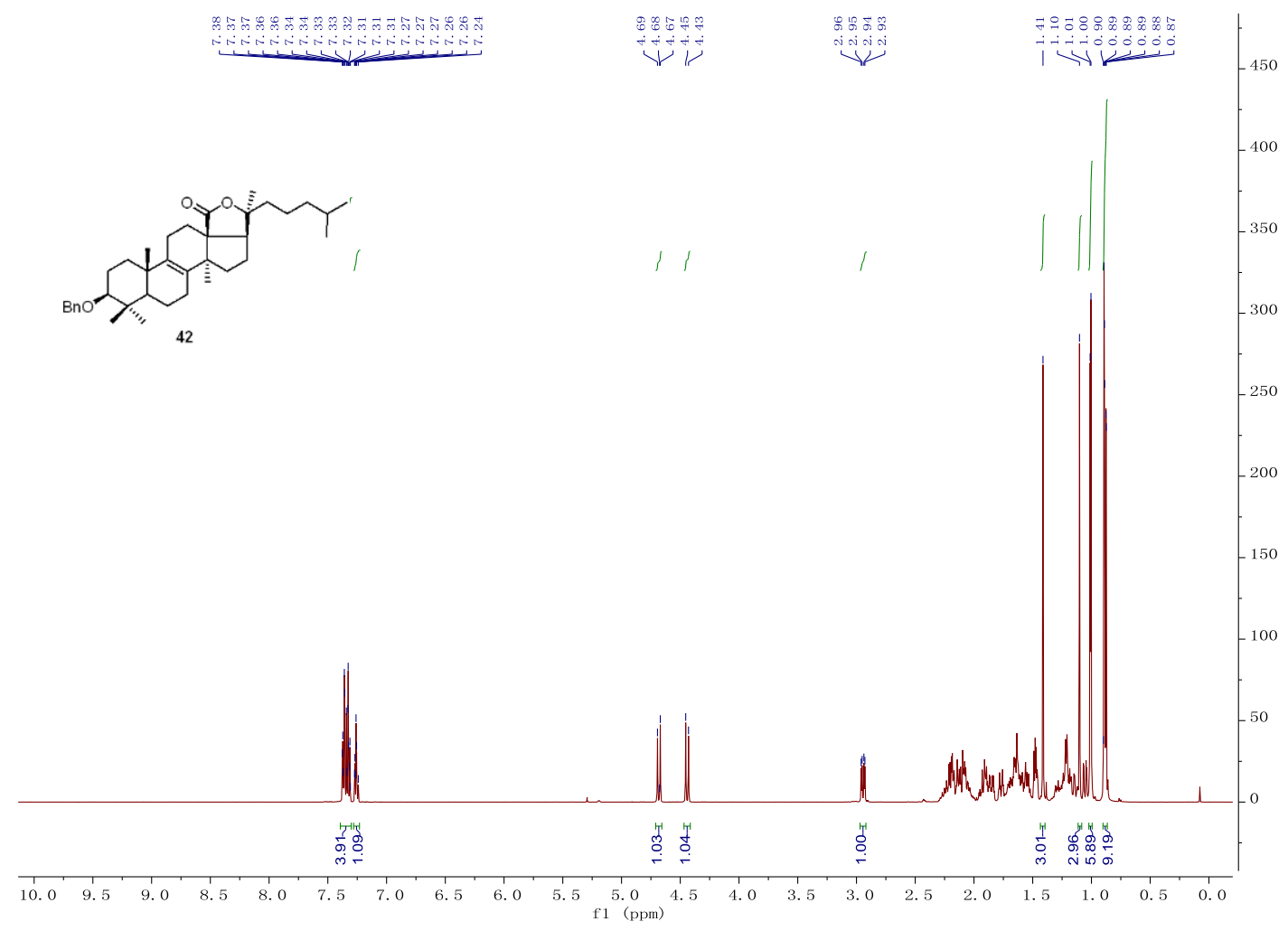

Figure S75. ${ }^{1} \mathrm{H}$ NMR spectrum of compound $42\left(500 \mathrm{MHz}, \mathrm{CDCl}_{3}\right)$

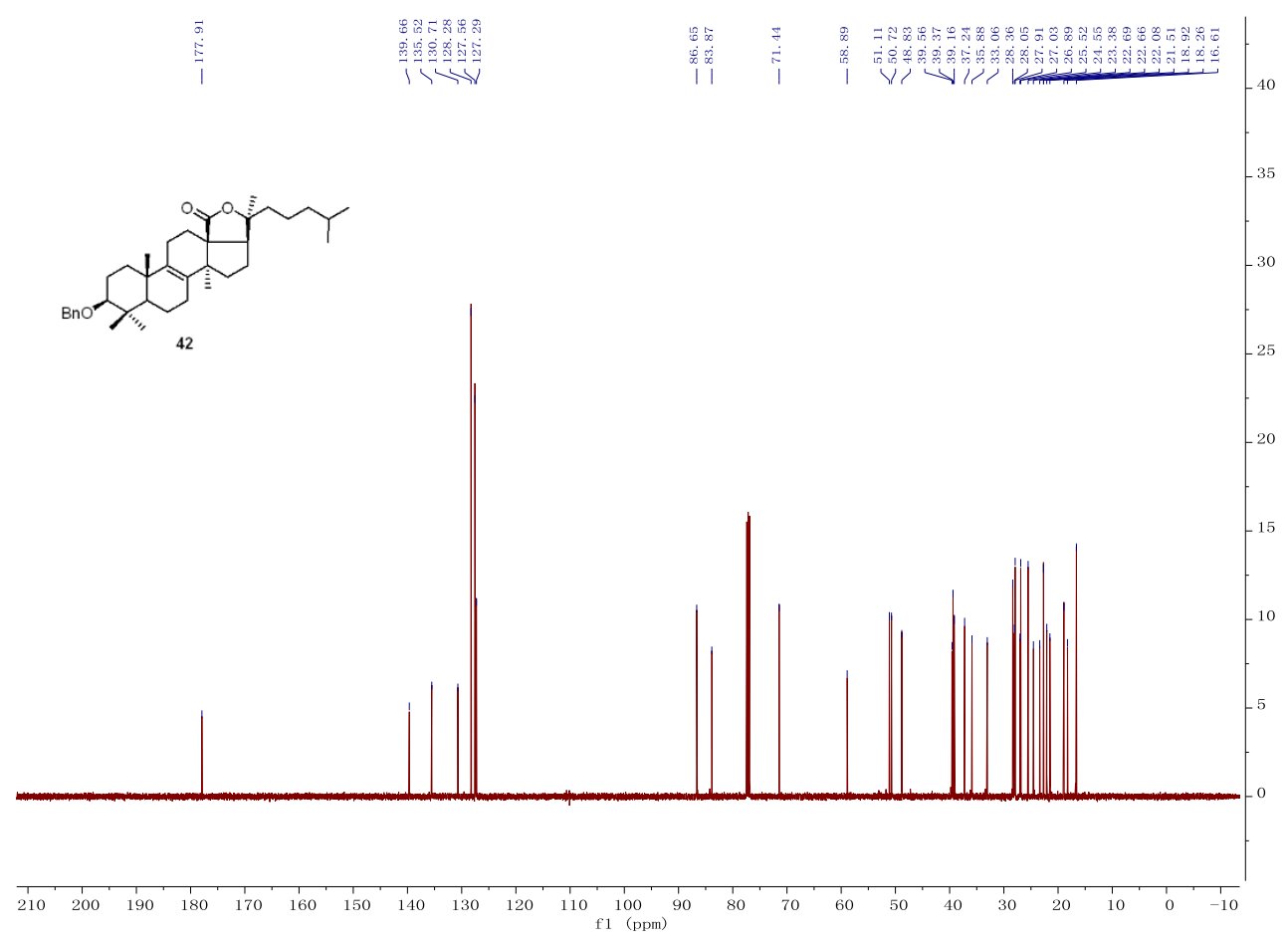

Figure S76. ${ }^{13} \mathrm{C}$ NMR spectrum of compound $42\left(125 \mathrm{MHz}, \mathrm{CDCl}_{3}\right)$ 


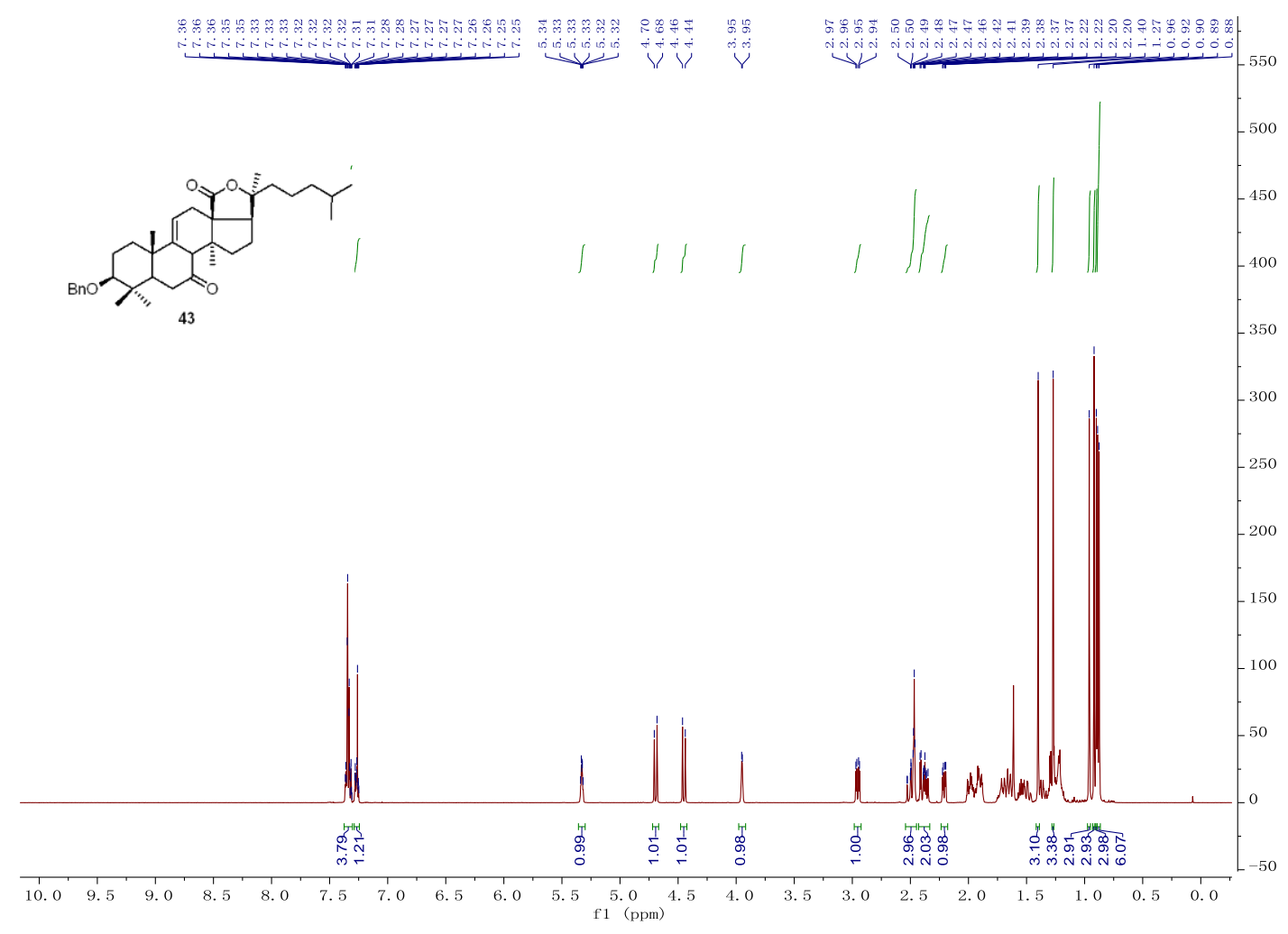

Figure S77. ${ }^{1} \mathrm{H}$ NMR spectrum of compound $43\left(500 \mathrm{MHz}, \mathrm{CDCl}_{3}\right)$

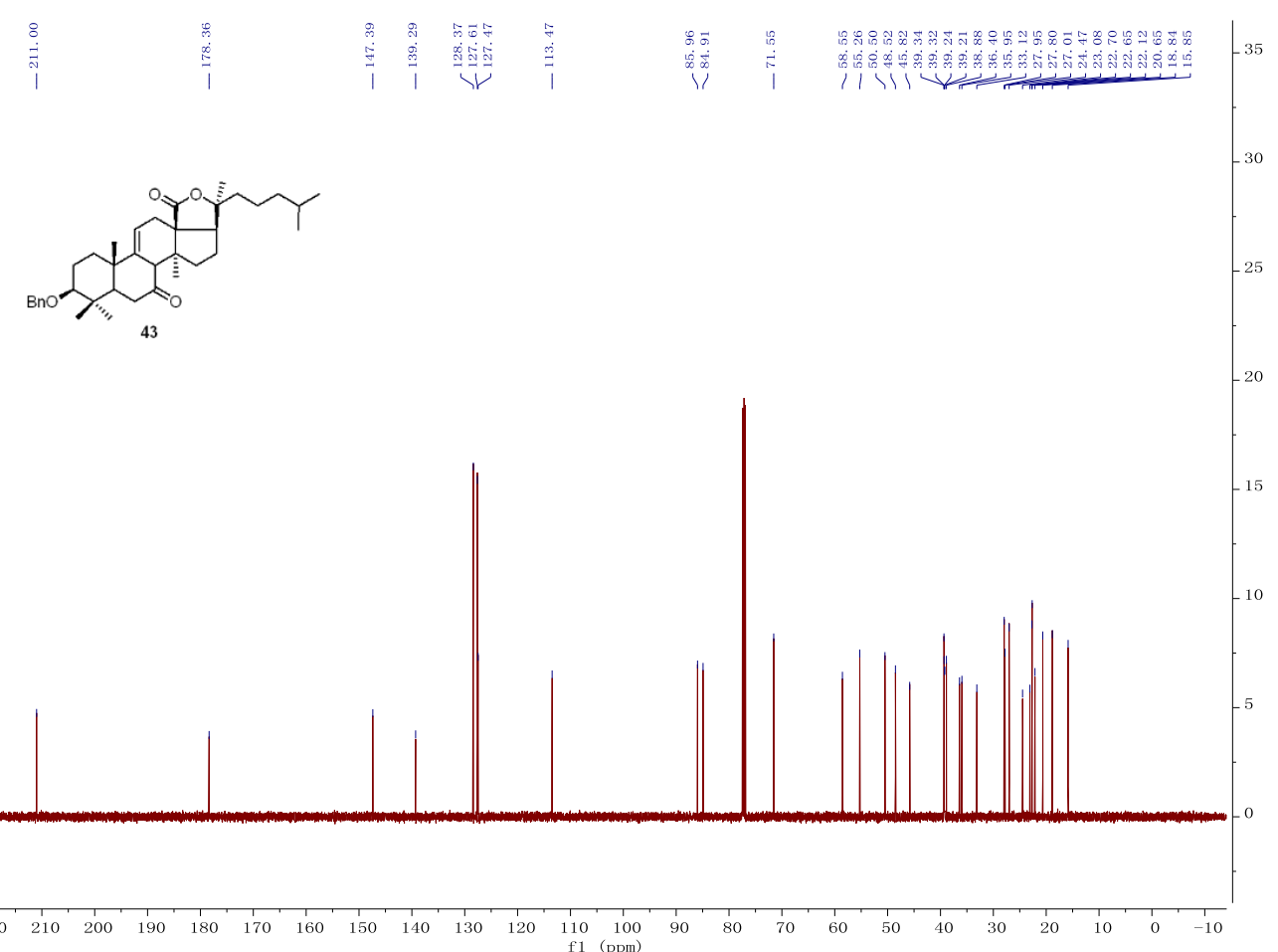

Figure S78. ${ }^{13} \mathrm{C}$ NMR spectrum of compound $43\left(125 \mathrm{MHz}, \mathrm{CDCl}_{3}\right)$ 


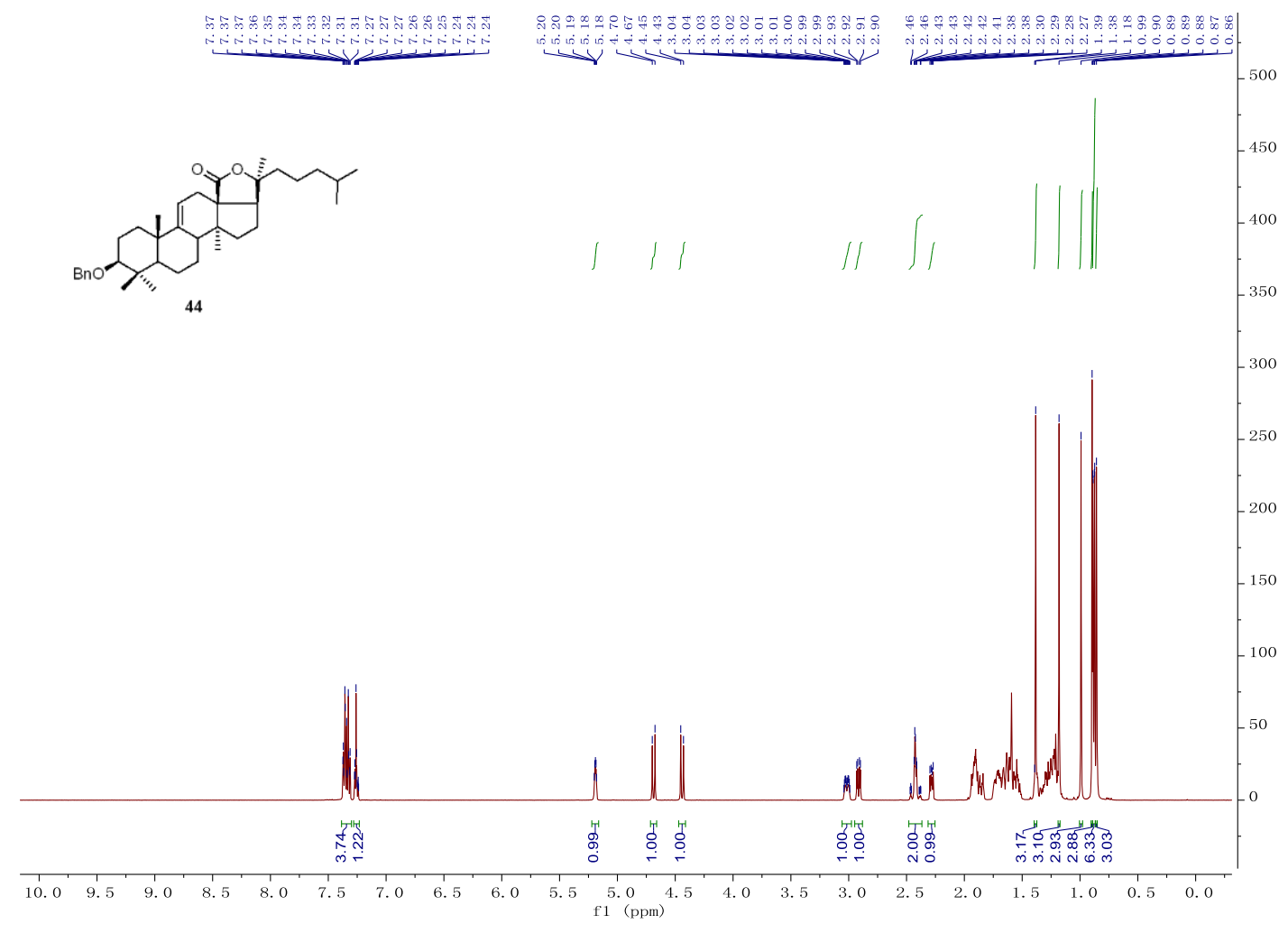

Figure S79. ${ }^{1} \mathrm{H}$ NMR spectrum of compound $44\left(500 \mathrm{MHz}, \mathrm{CDCl}_{3}\right)$

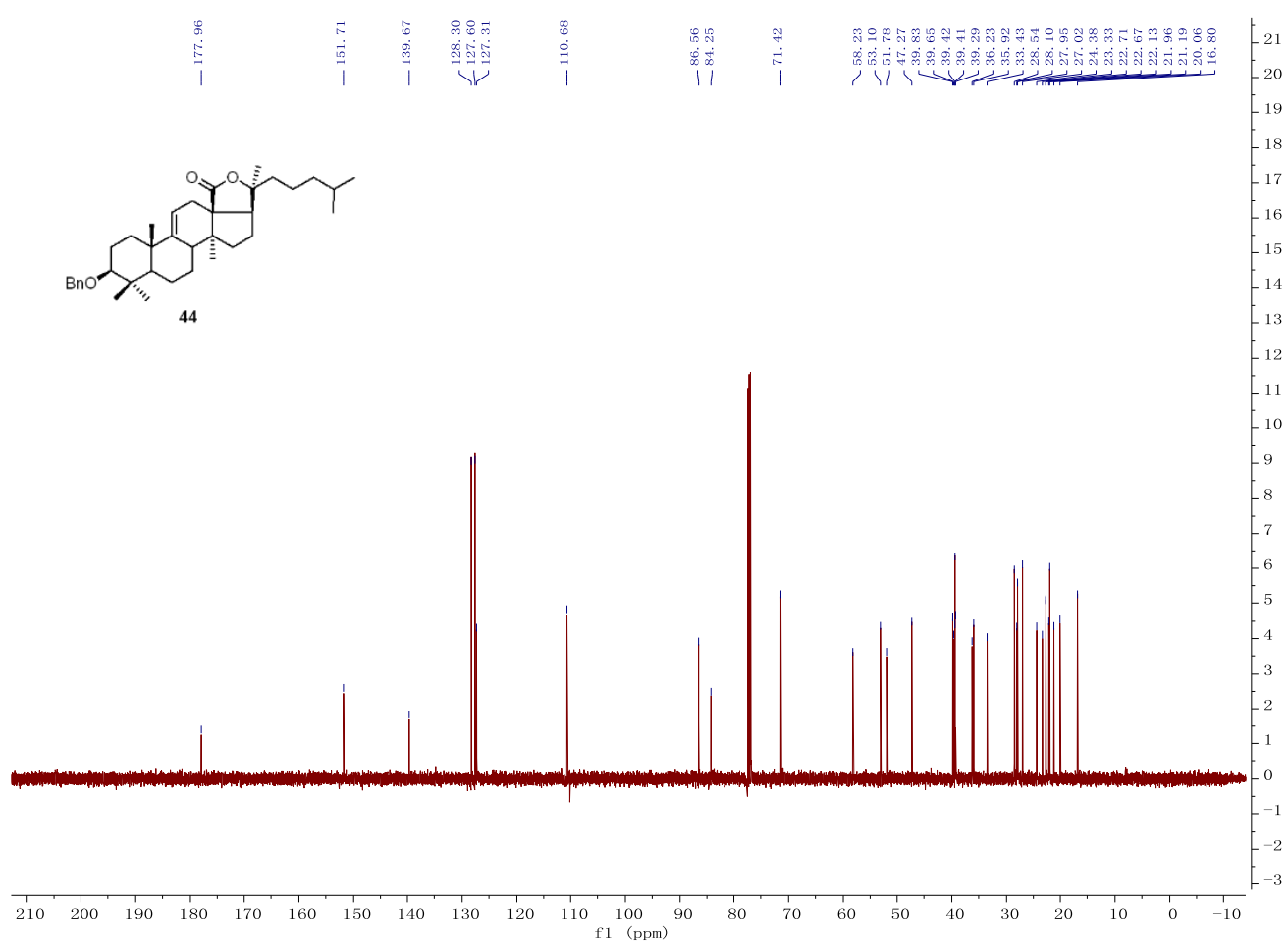

Figure S80. ${ }^{13} \mathrm{C}$ NMR spectrum of compound $44\left(125 \mathrm{MHz}, \mathrm{CDCl}_{3}\right)$ 


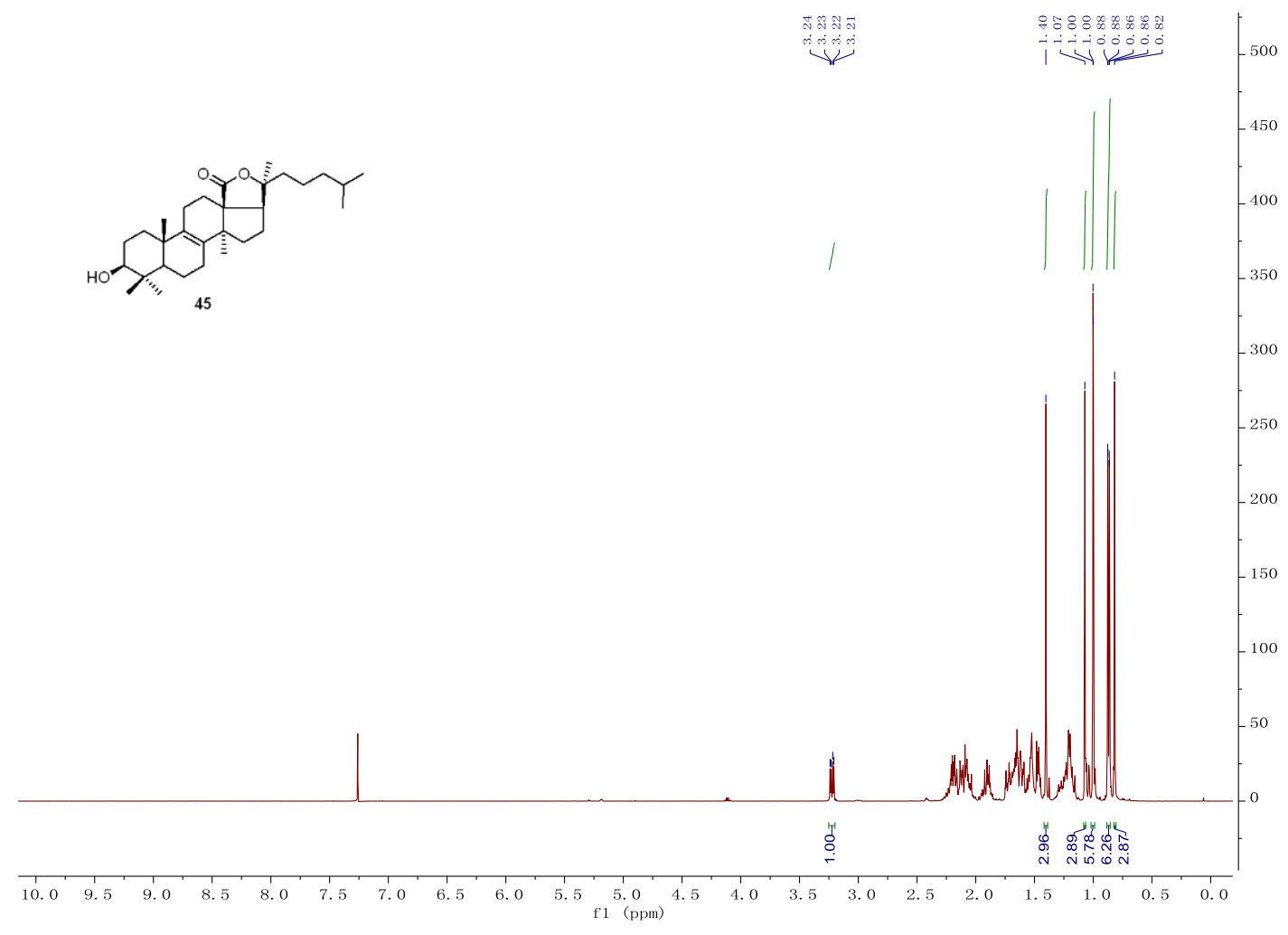

Figure S81. ${ }^{1} \mathrm{H}$ NMR spectrum of compound $45\left(500 \mathrm{MHz}, \mathrm{CDCl}_{3}\right)$

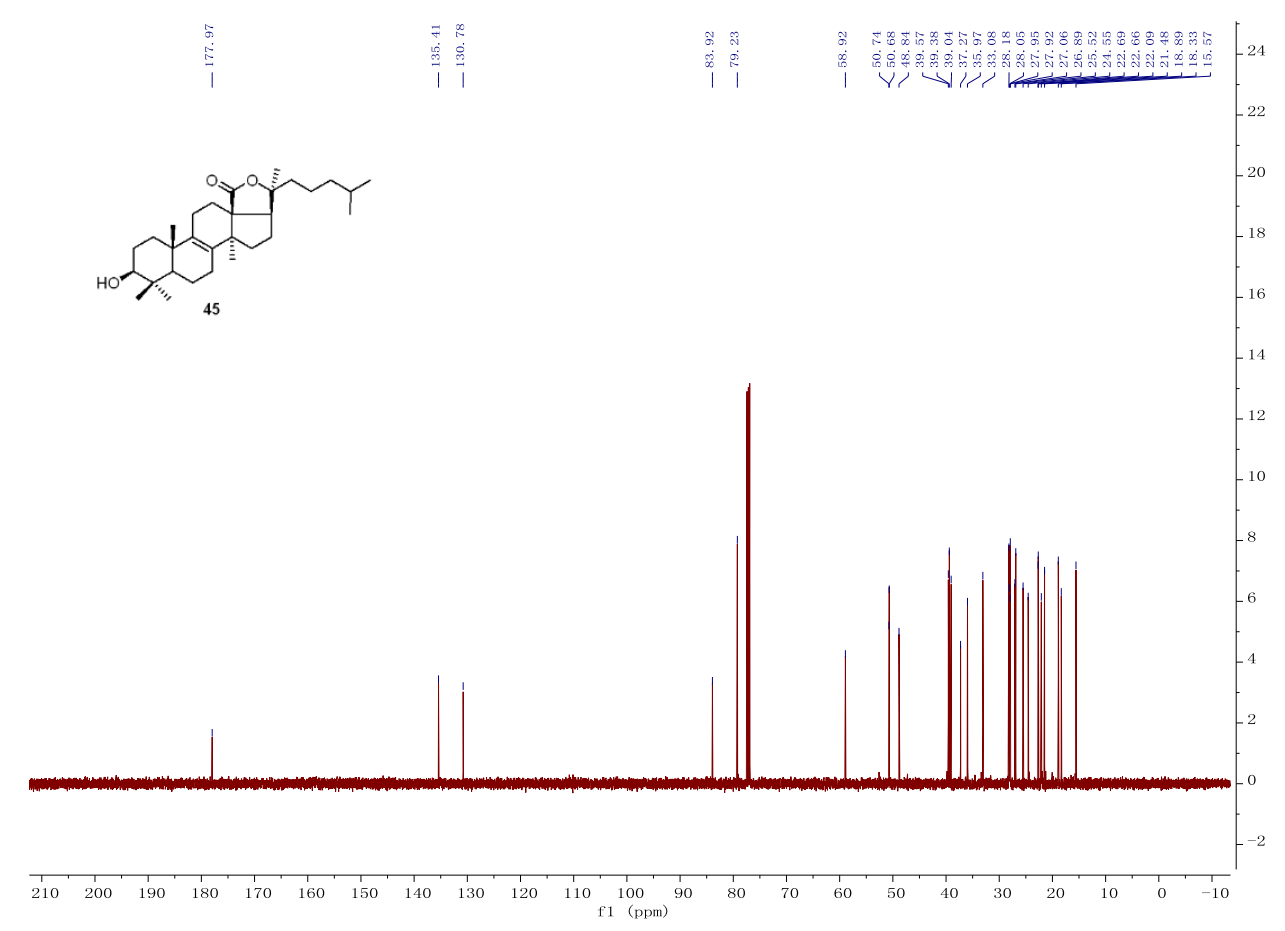

Figure S82. ${ }^{13} \mathrm{C}$ NMR spectrum of compound $45\left(125 \mathrm{MHz}, \mathrm{CDCl}_{3}\right)$ 


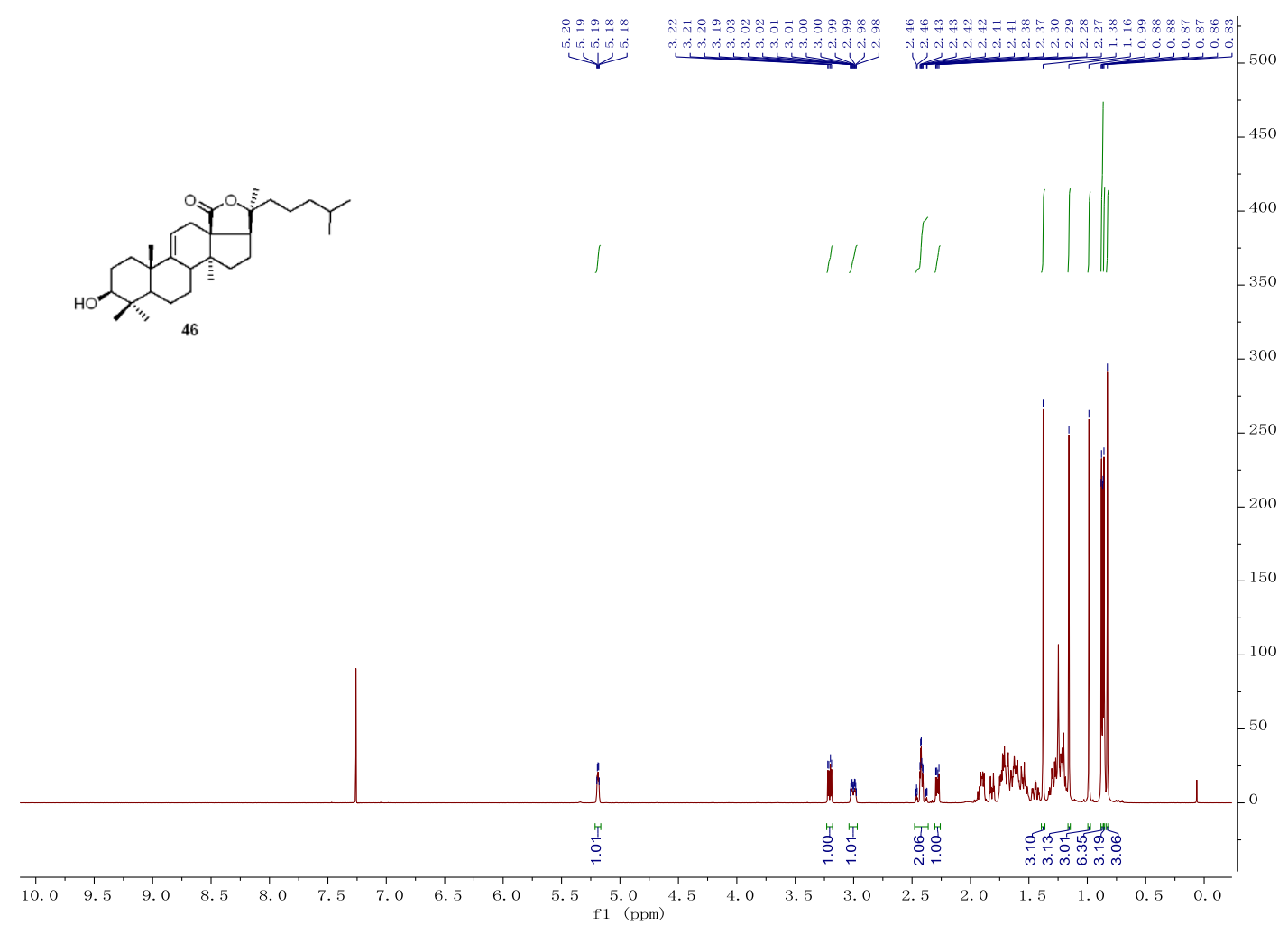

Figure S83. ${ }^{1} \mathrm{H}$ NMR spectrum of compound $46\left(500 \mathrm{MHz}, \mathrm{CDCl}_{3}\right)$

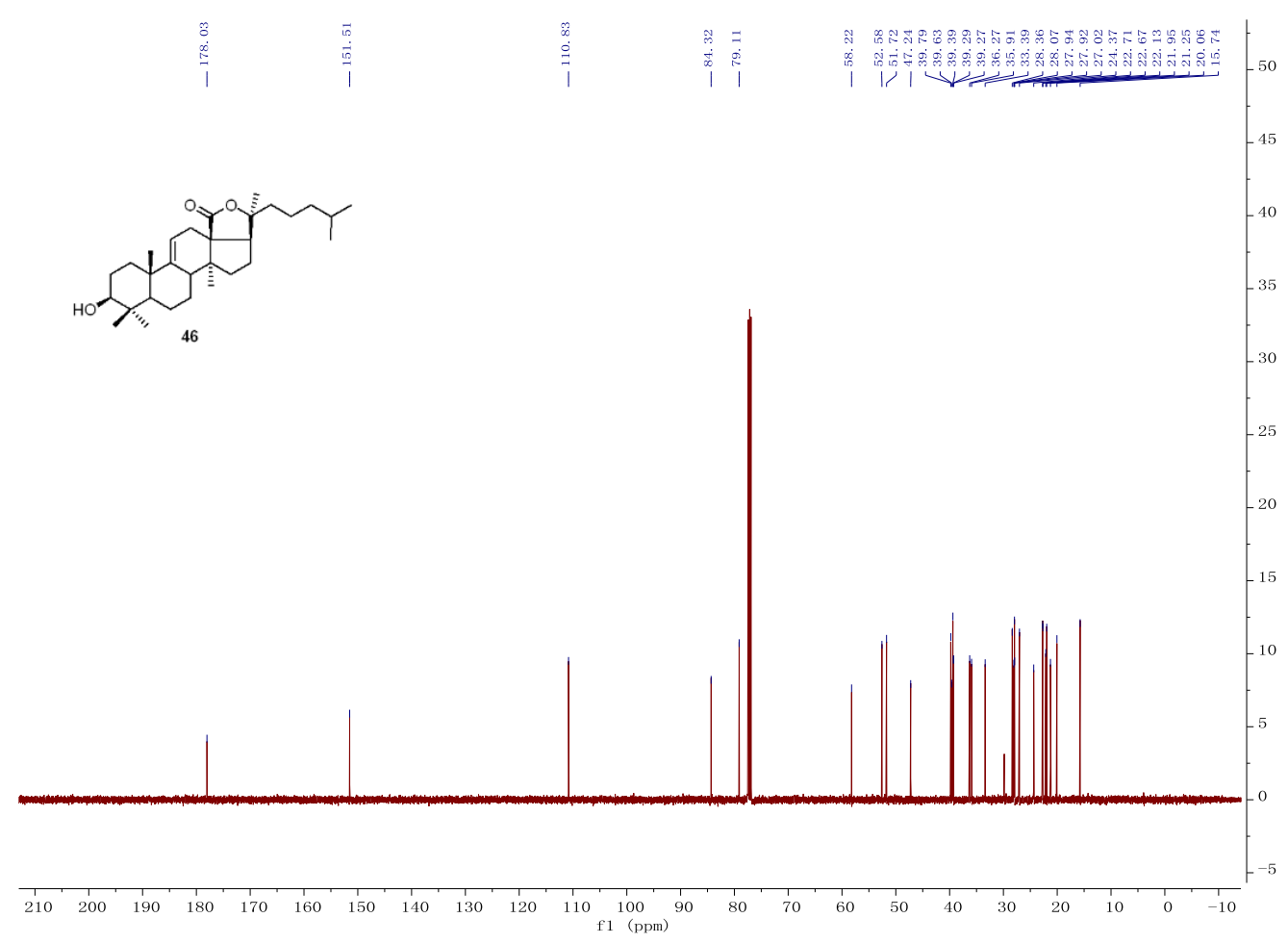

Figure S84. ${ }^{13} \mathrm{C}$ NMR spectrum of compound $46\left(125 \mathrm{MHz}, \mathrm{CDCl}_{3}\right)$ 


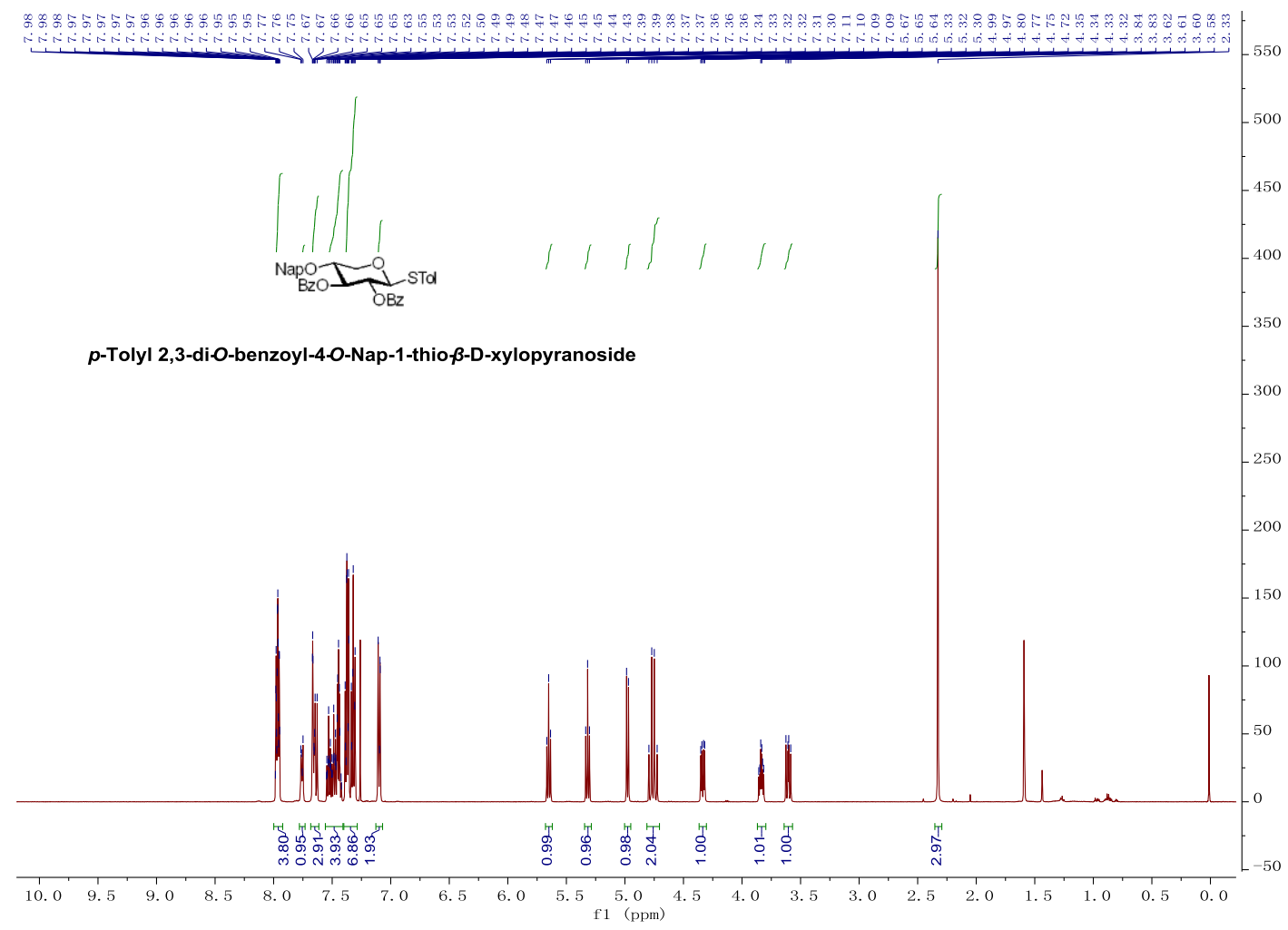

Figure S85. ${ }^{1} \mathrm{H}$ NMR spectrum of $p$-tolyl 2,3-di- $O$-benzoyl-4- $O$-Nap-1-thio- $\beta$-Dxylopyranoside $\left(500 \mathrm{MHz}, \mathrm{CDCl}_{3}\right)$

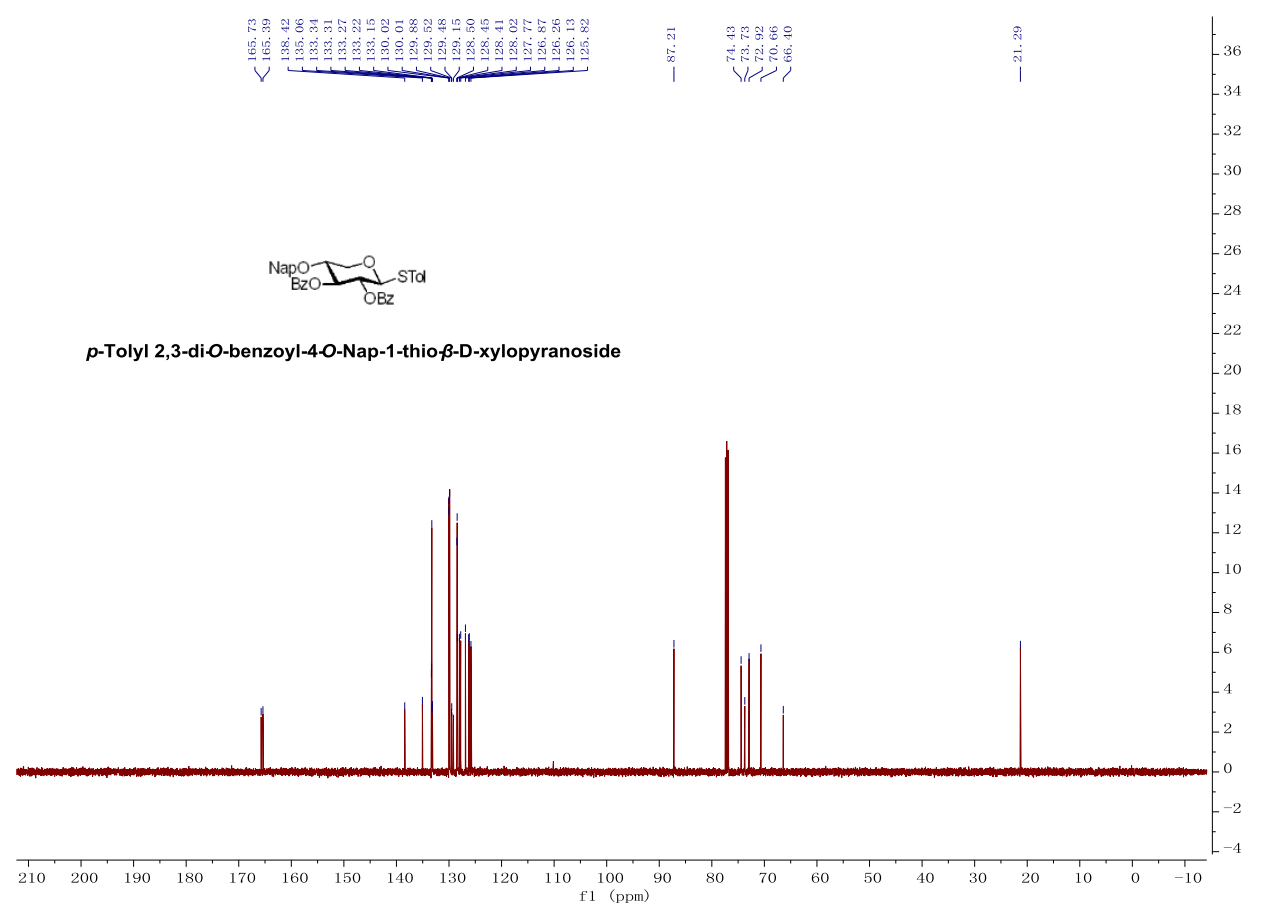

Figure S86. ${ }^{13} \mathrm{C}$ NMR spectrum of $p$-tolyl 2,3-di- $O$-benzoyl-4- $O$-Nap-1-thio- $\beta$-Dxylopyranoside $\left(125 \mathrm{MHz}, \mathrm{CDCl}_{3}\right)$ 


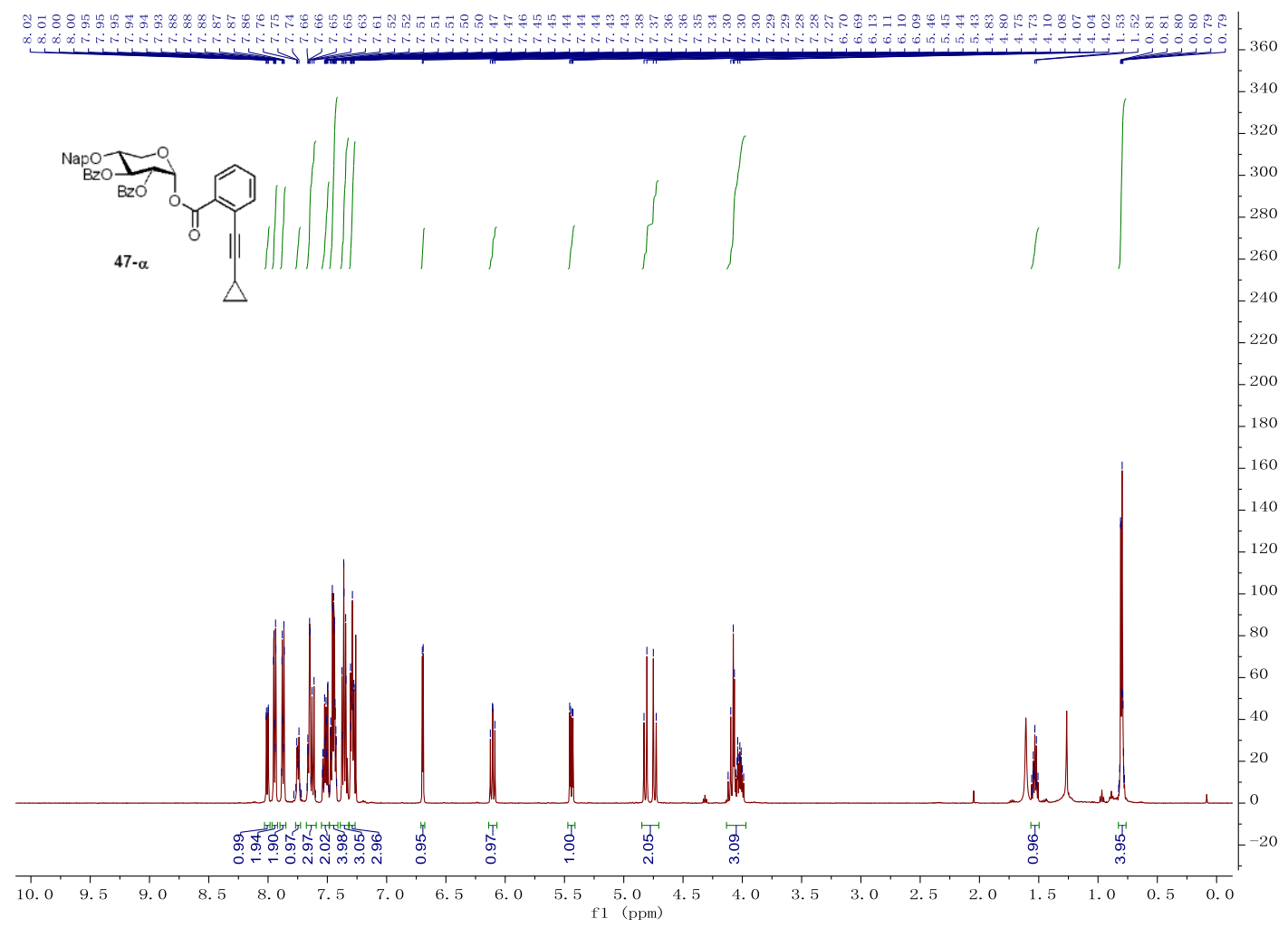

Figure S87. ${ }^{1} \mathrm{H}$ NMR spectrum of compound $47 \boldsymbol{\alpha}\left(500 \mathrm{MHz}, \mathrm{CDCl}_{3}\right)$

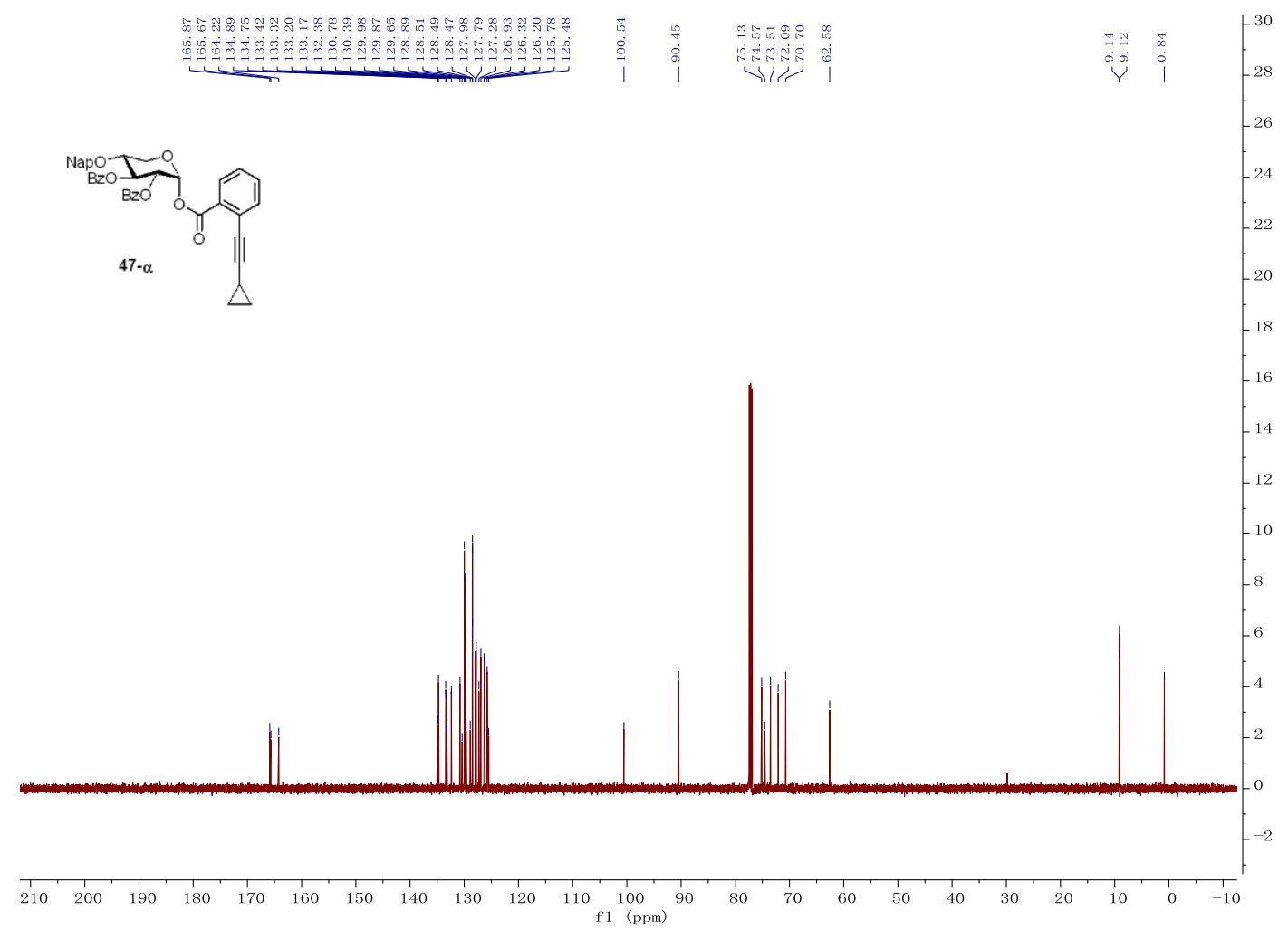

Figure S88. ${ }^{13} \mathrm{C}$ NMR spectrum of compound $47 \alpha\left(125 \mathrm{MHz}, \mathrm{CDCl}_{3}\right)$ 


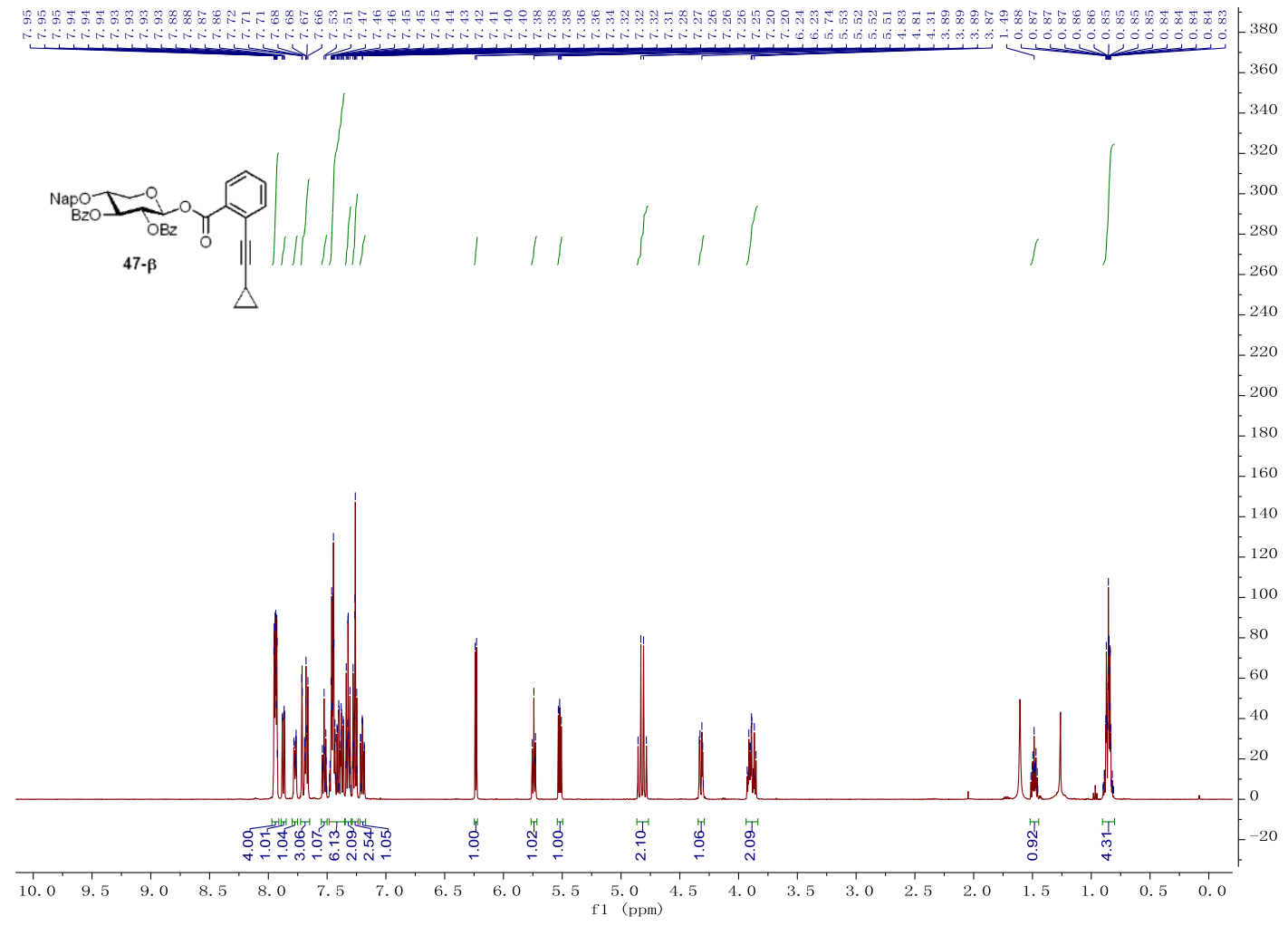

Figure S89. ${ }^{1} \mathrm{H}$ NMR spectrum of compound $47 \boldsymbol{\beta}\left(500 \mathrm{MHz}, \mathrm{CDCl}_{3}\right)$

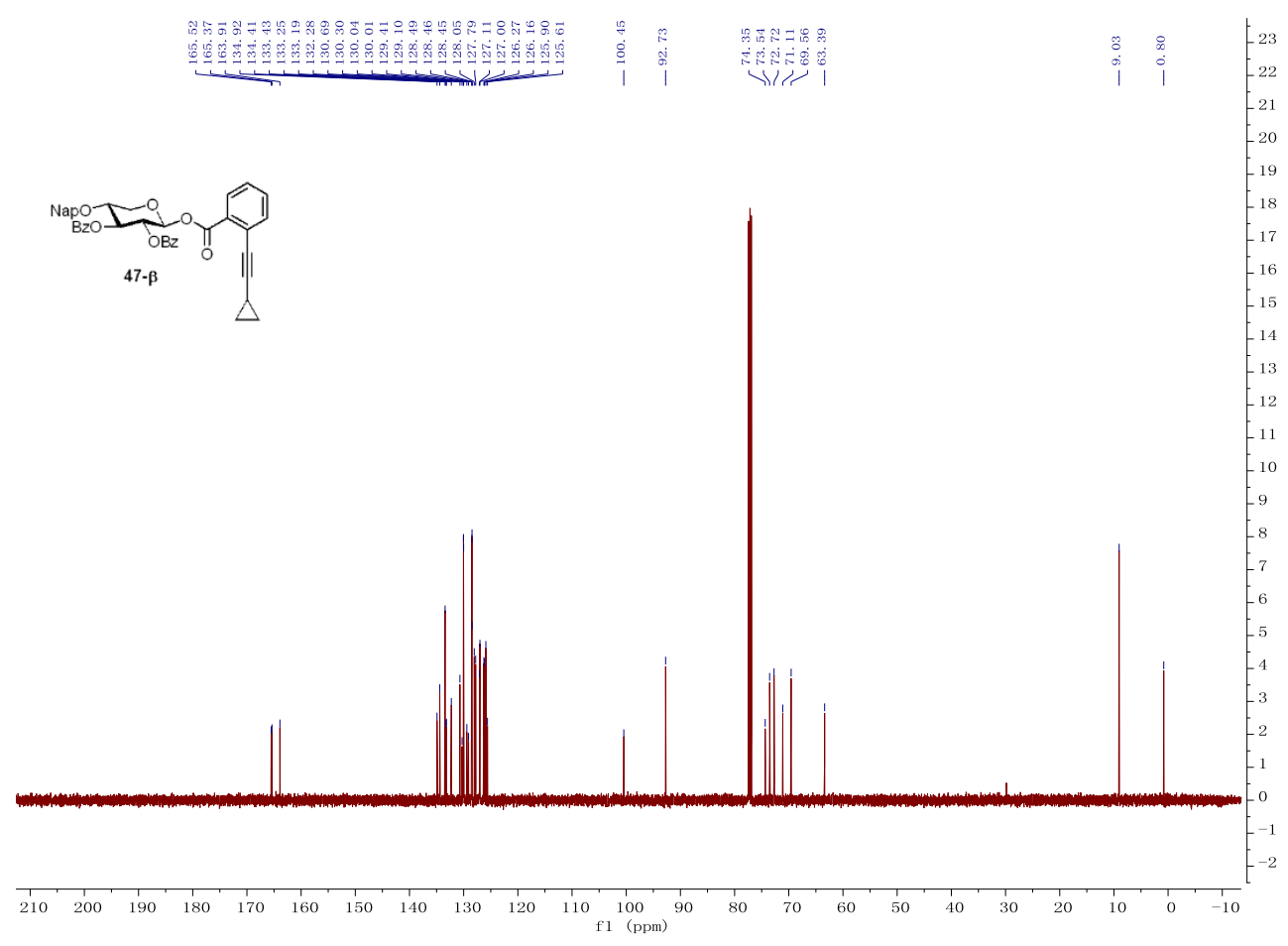

Figure S90. ${ }^{13} \mathrm{C}$ NMR spectrum of compound $47 \boldsymbol{\beta}\left(125 \mathrm{MHz}, \mathrm{CDCl}_{3}\right)$ 


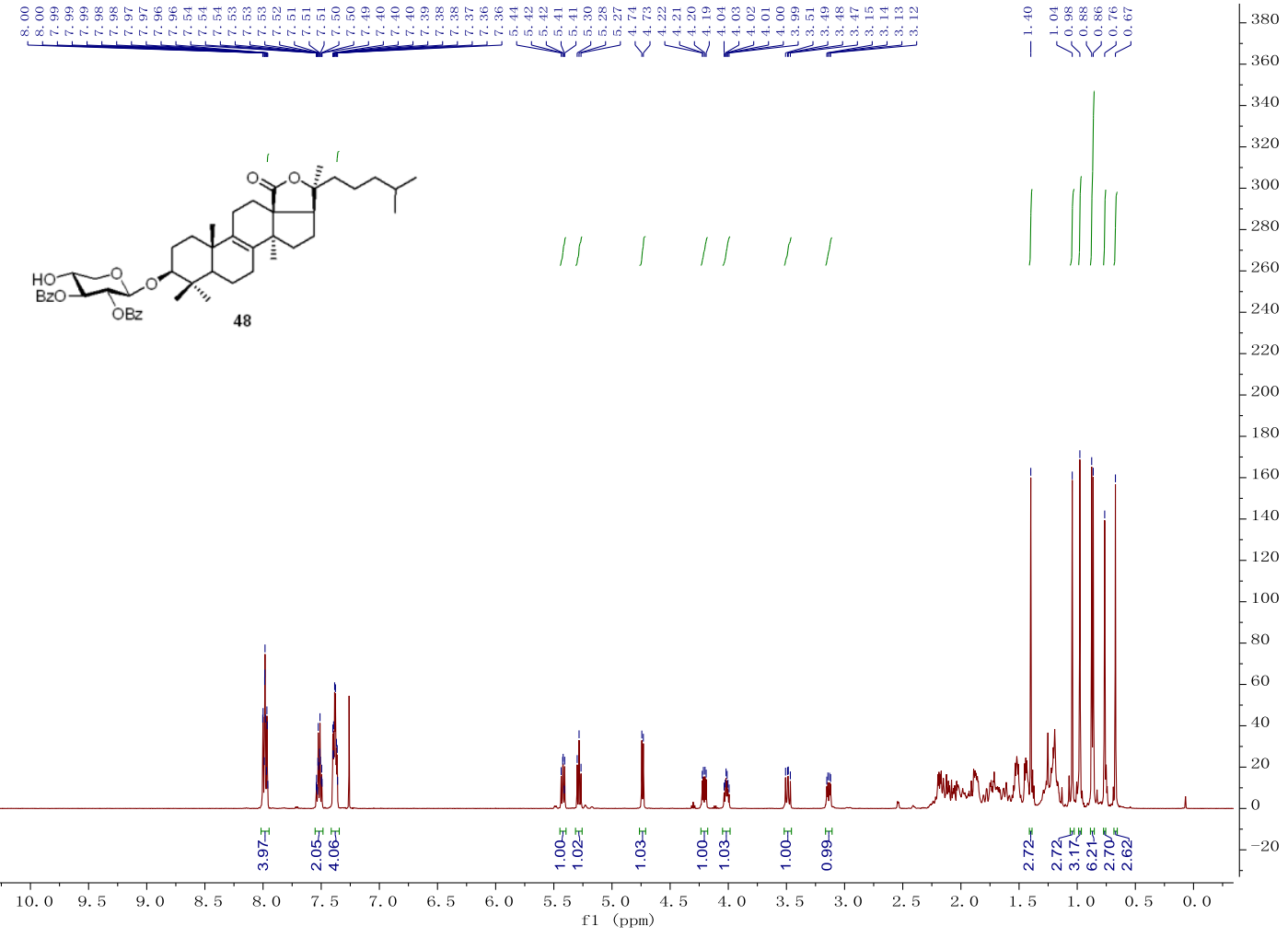

Figure S91. ${ }^{1} \mathrm{H}$ NMR spectrum of compound $48\left(500 \mathrm{MHz}, \mathrm{CDCl}_{3}\right)$

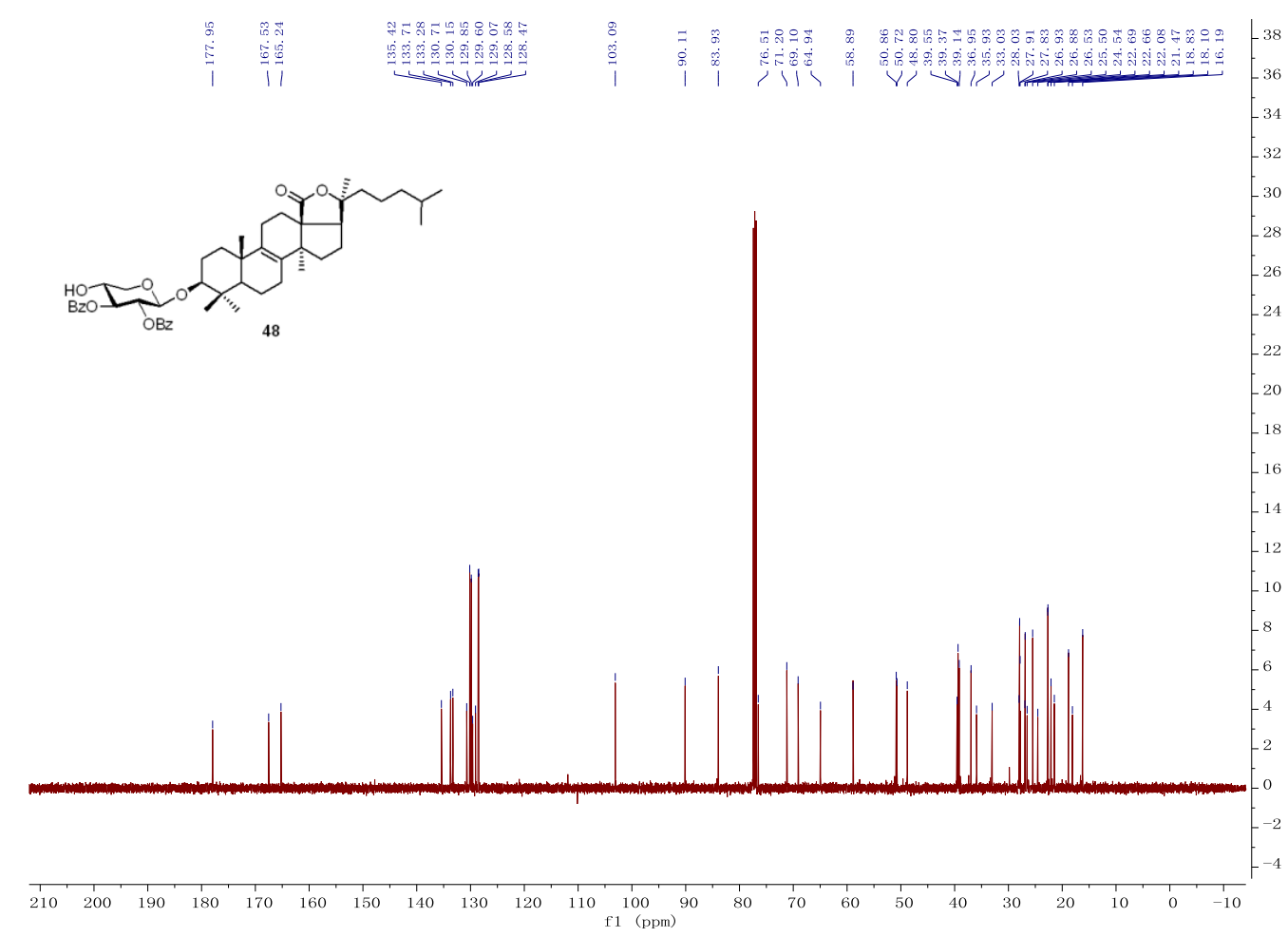

Figure S92. ${ }^{13} \mathrm{C}$ NMR spectrum of compound $48\left(125 \mathrm{MHz}, \mathrm{CDCl}_{3}\right)$ 


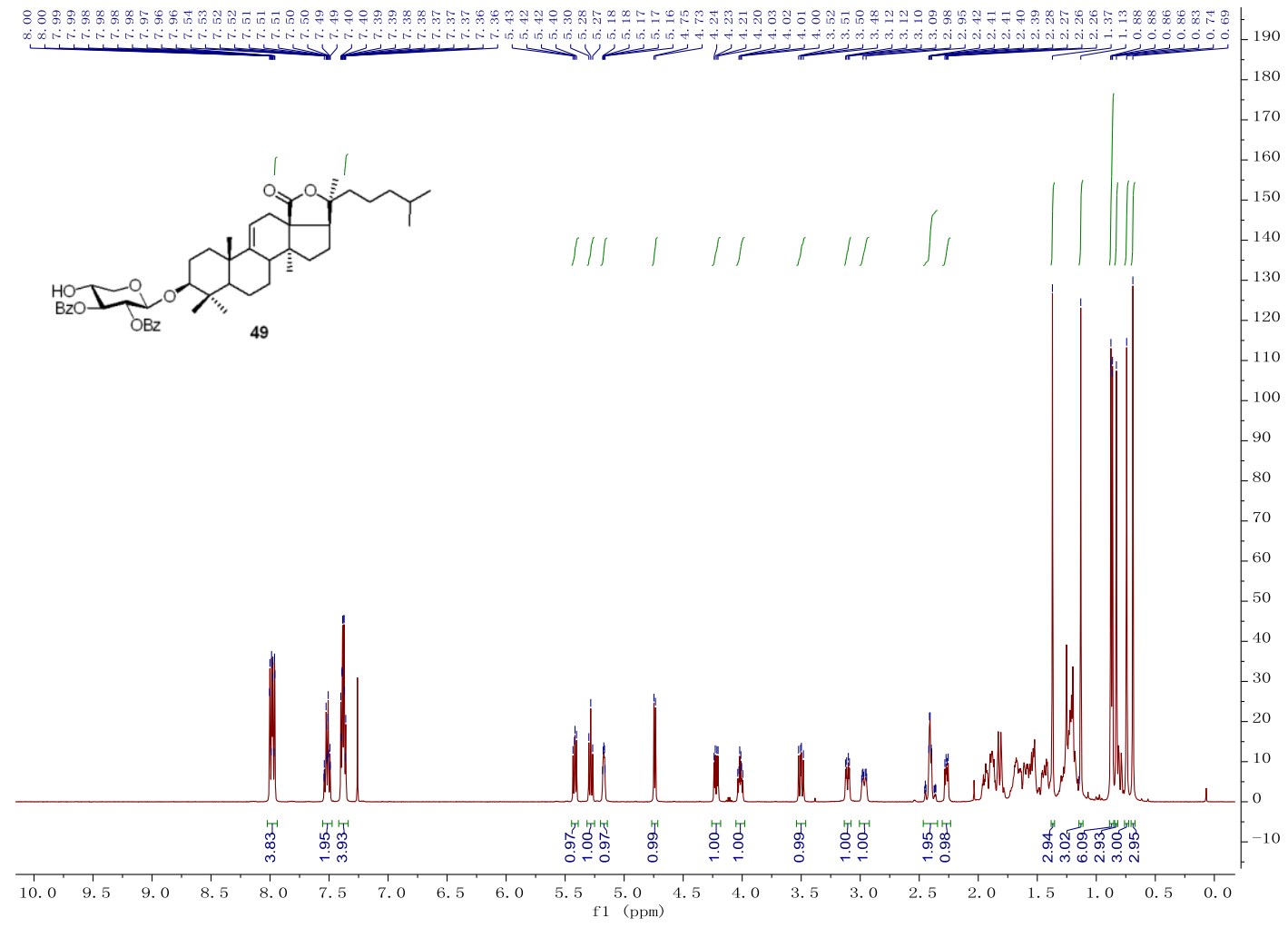

Figure S93. ${ }^{1} \mathrm{H}$ NMR spectrum of compound $49\left(500 \mathrm{MHz}, \mathrm{CDCl}_{3}\right)$

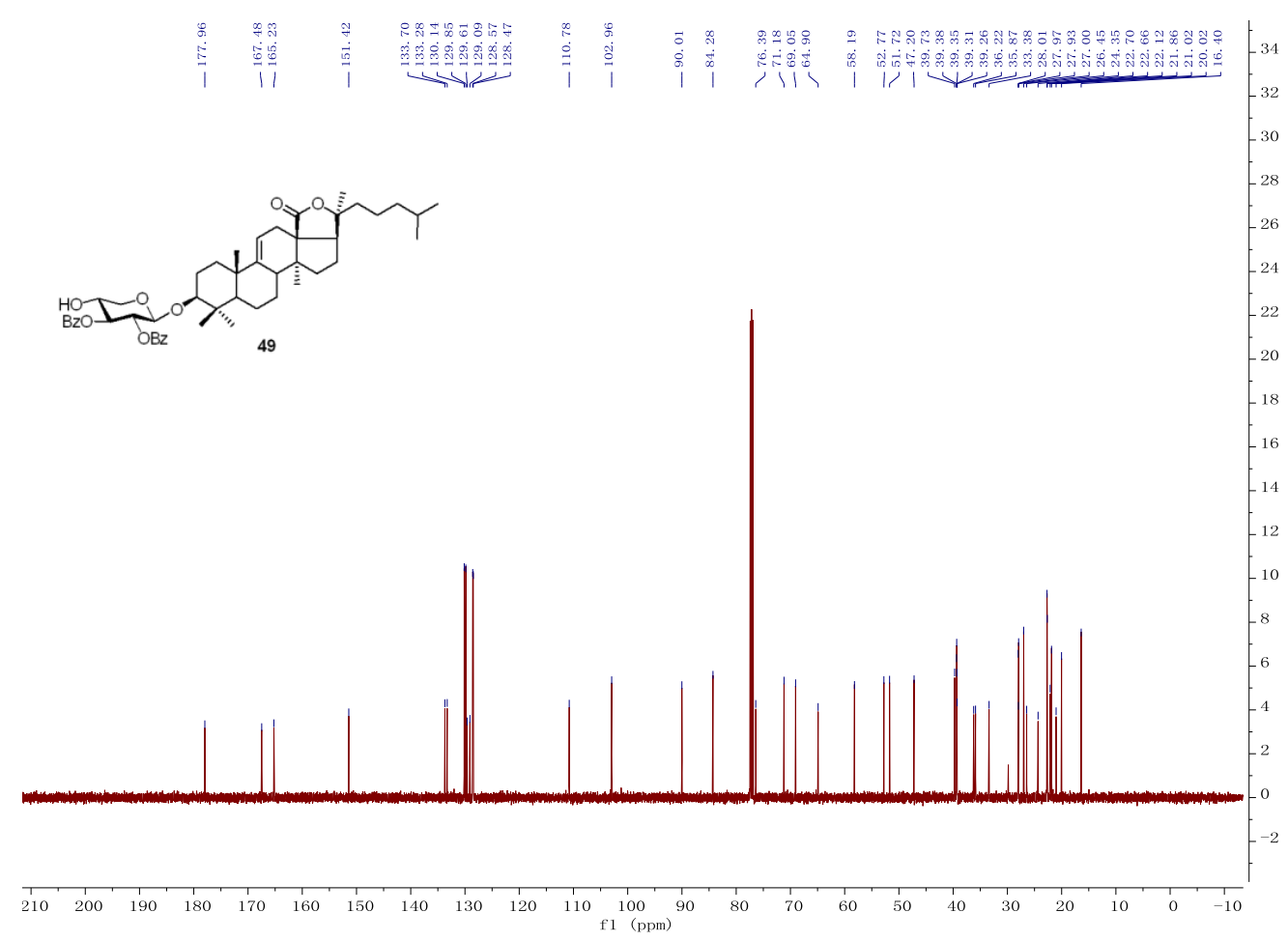

Figure S94. ${ }^{13} \mathrm{C}$ NMR spectrum of compound $49\left(125 \mathrm{MHz}, \mathrm{CDCl}_{3}\right)$ 


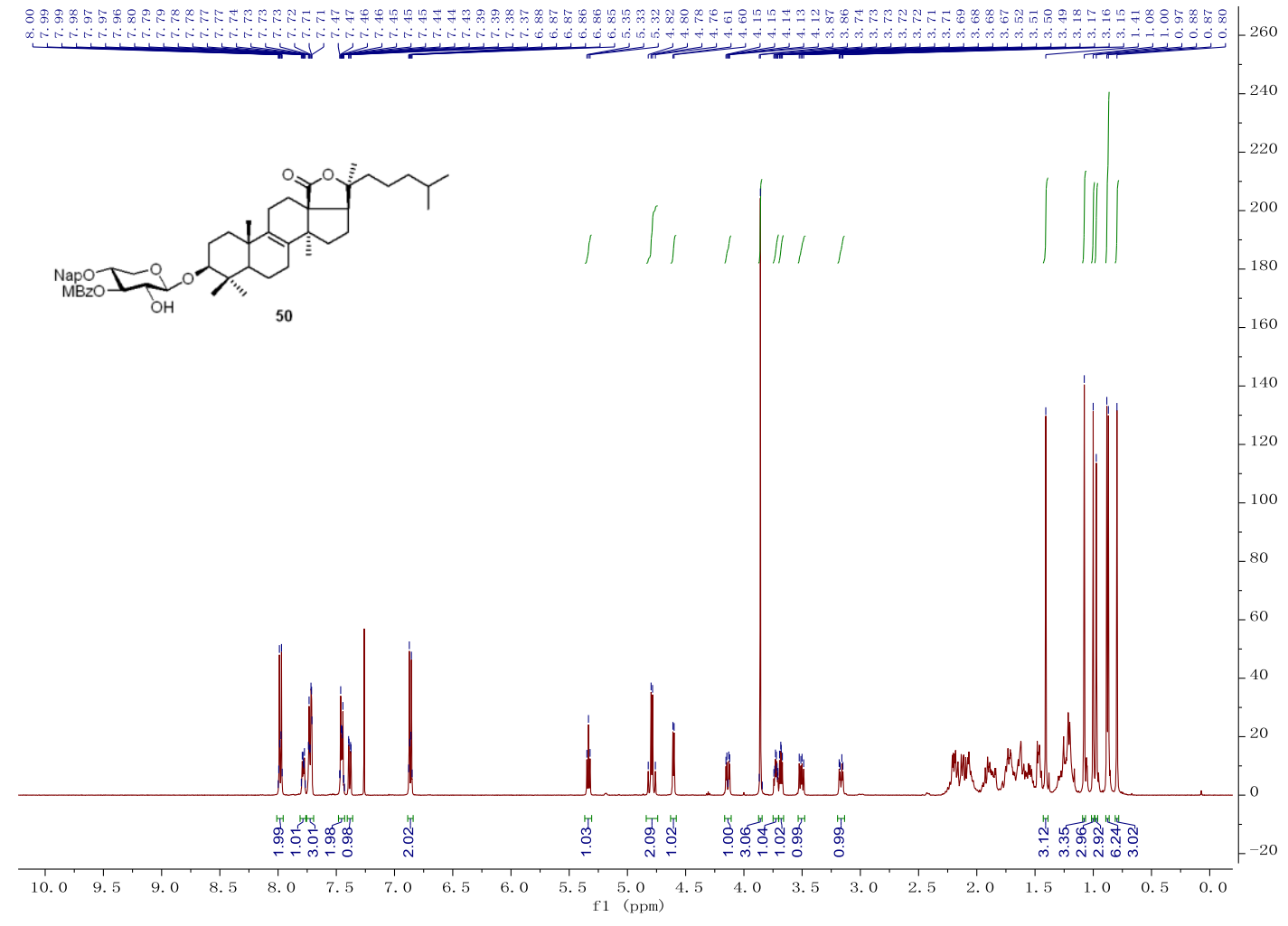

Figure S95. ${ }^{1} \mathrm{H}$ NMR spectrum of compound $50\left(500 \mathrm{MHz}, \mathrm{CDCl}_{3}\right)$

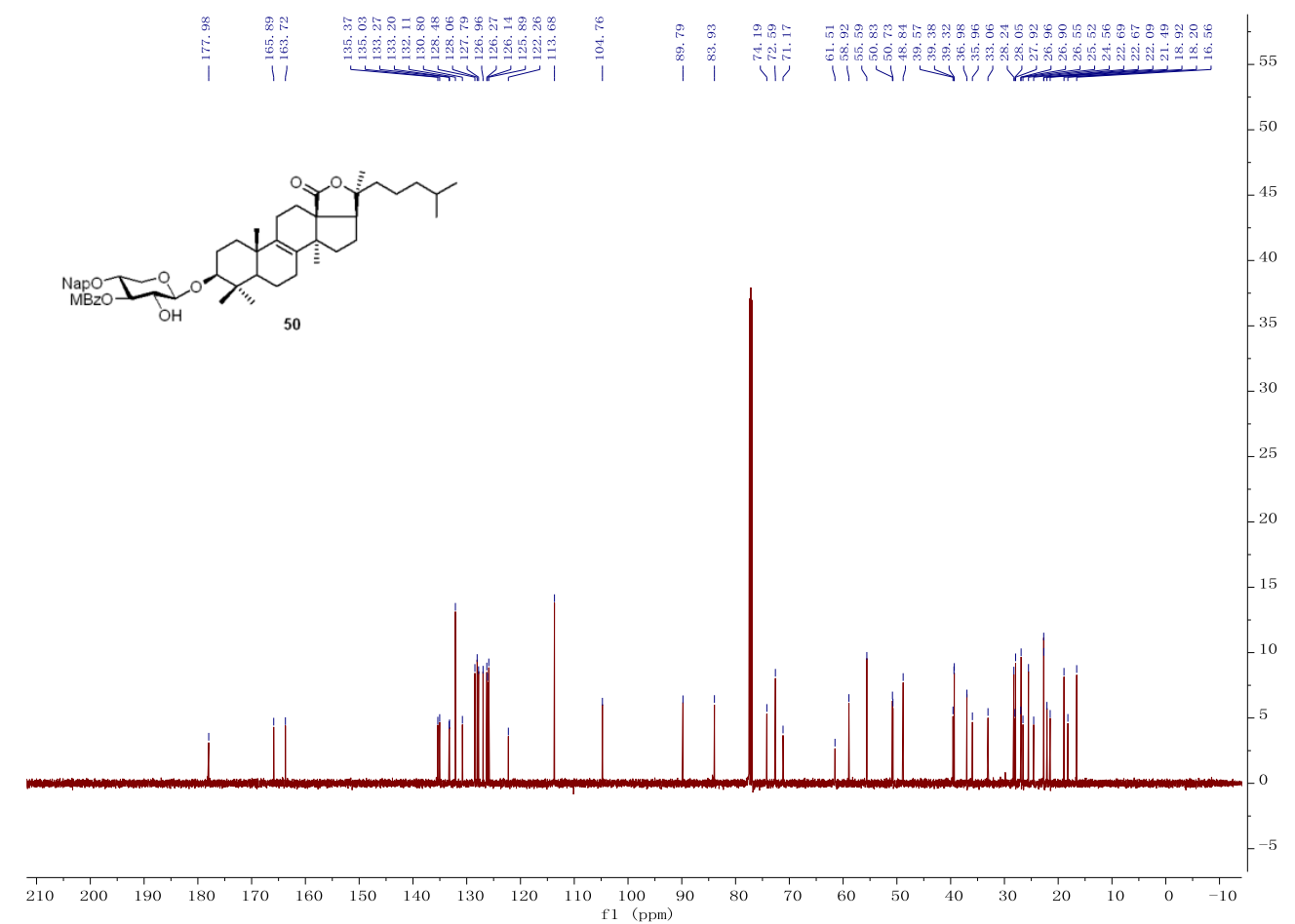

Figure S96. ${ }^{13} \mathrm{C}$ NMR spectrum of compound $50\left(125 \mathrm{MHz}, \mathrm{CDCl}_{3}\right)$ 


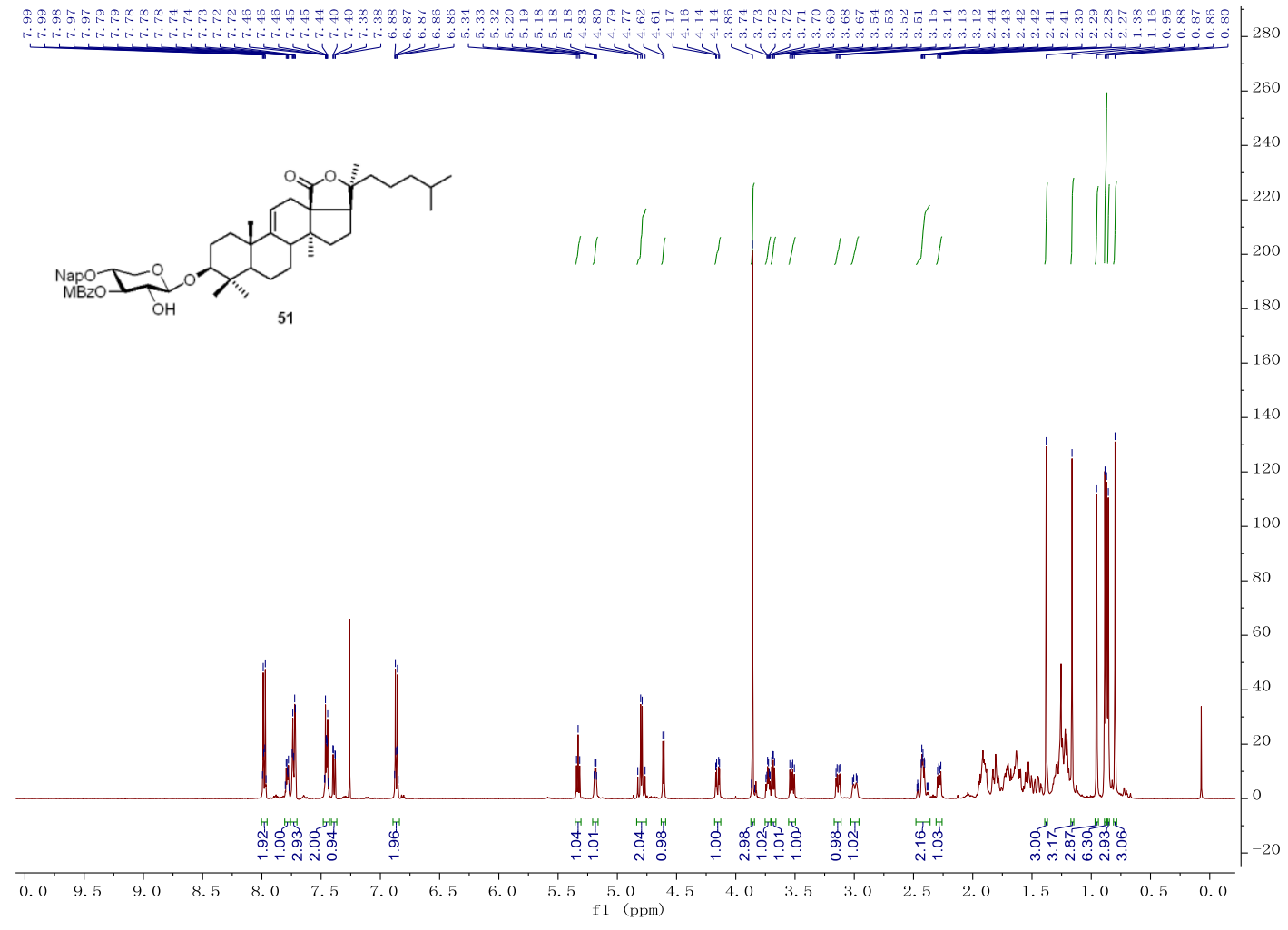

Figure S97. ${ }^{1} \mathrm{H}$ NMR spectrum of compound $51\left(500 \mathrm{MHz}, \mathrm{CDCl}_{3}\right)$

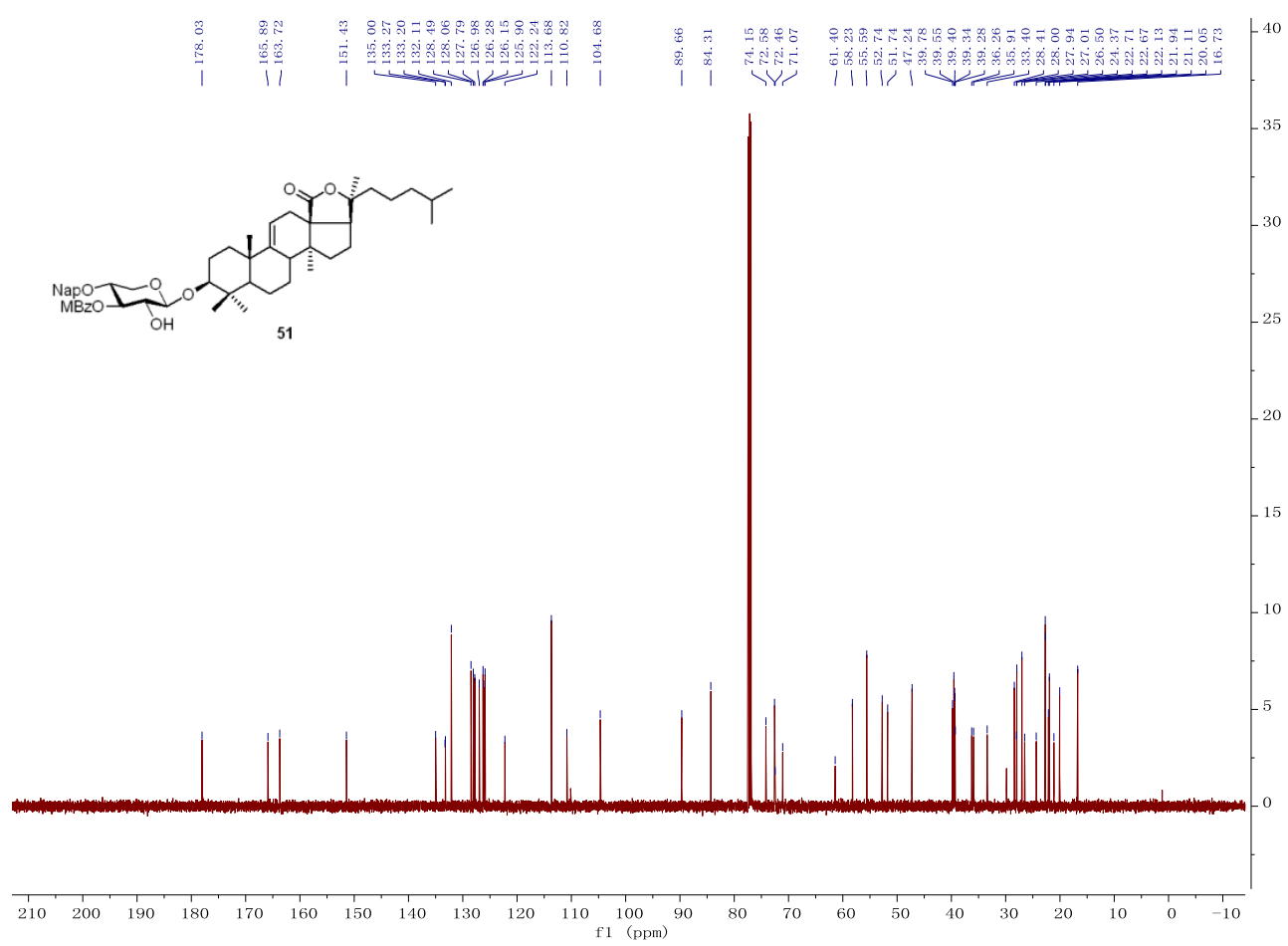

Figure S98. ${ }^{13} \mathrm{C}$ NMR spectrum of compound $51\left(125 \mathrm{MHz}, \mathrm{CDCl}_{3}\right)$ 


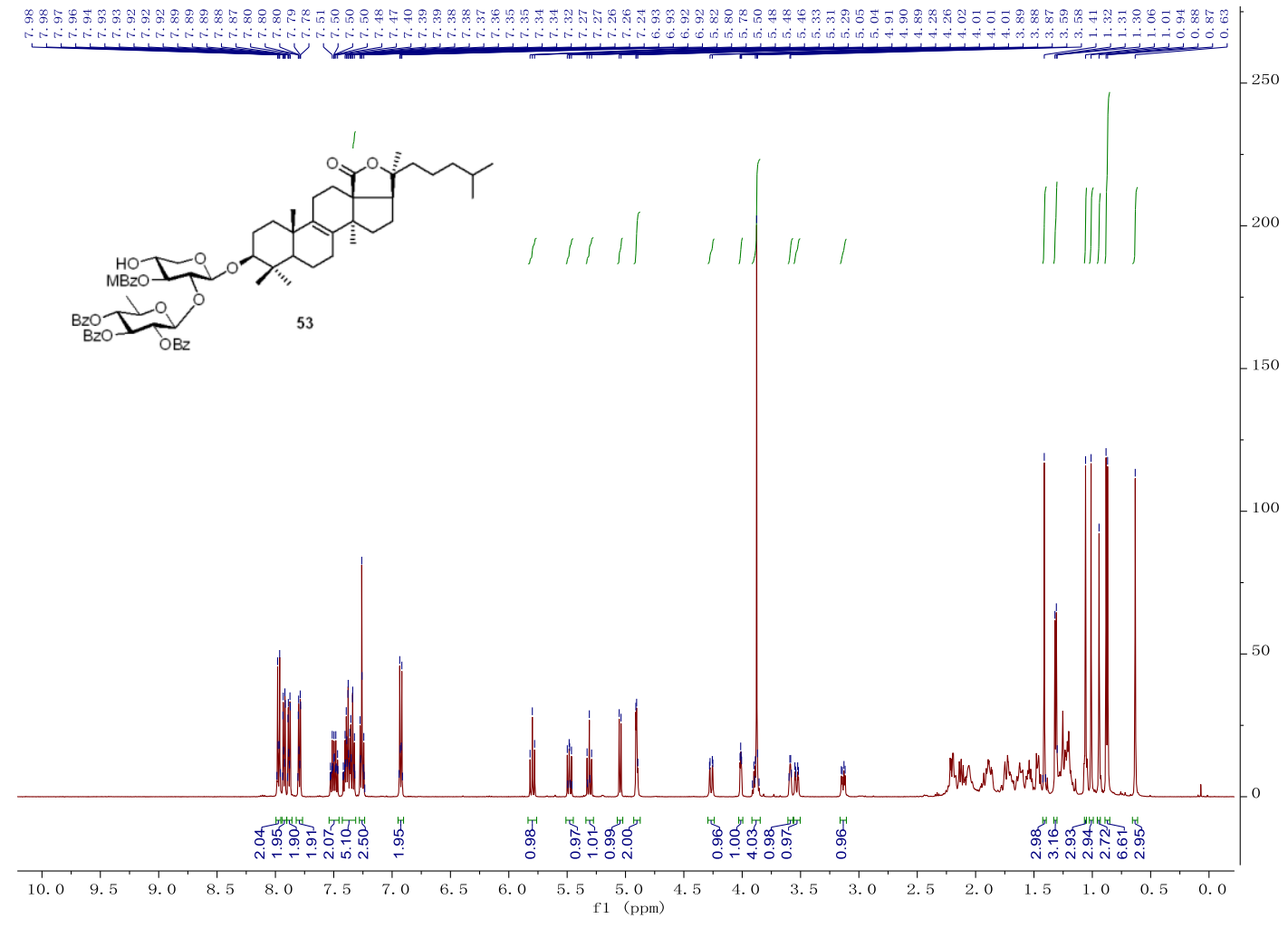

Figure S99. ${ }^{1} \mathrm{H}$ NMR spectrum of compound $53\left(500 \mathrm{MHz}, \mathrm{CDCl}_{3}\right)$

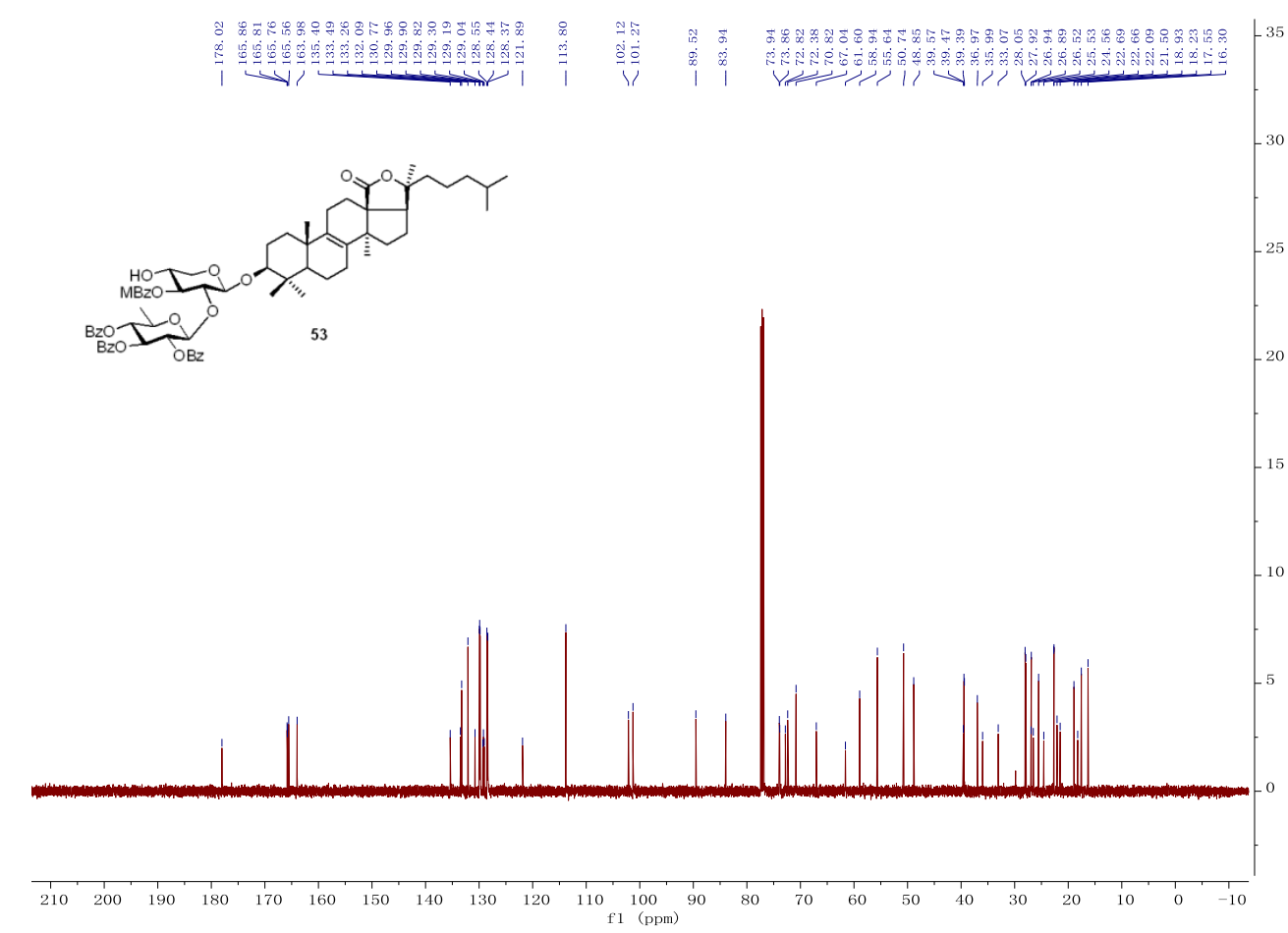

Figure S100. ${ }^{13} \mathrm{C}$ NMR spectrum of compound $53\left(125 \mathrm{MHz}, \mathrm{CDCl}_{3}\right)$ 


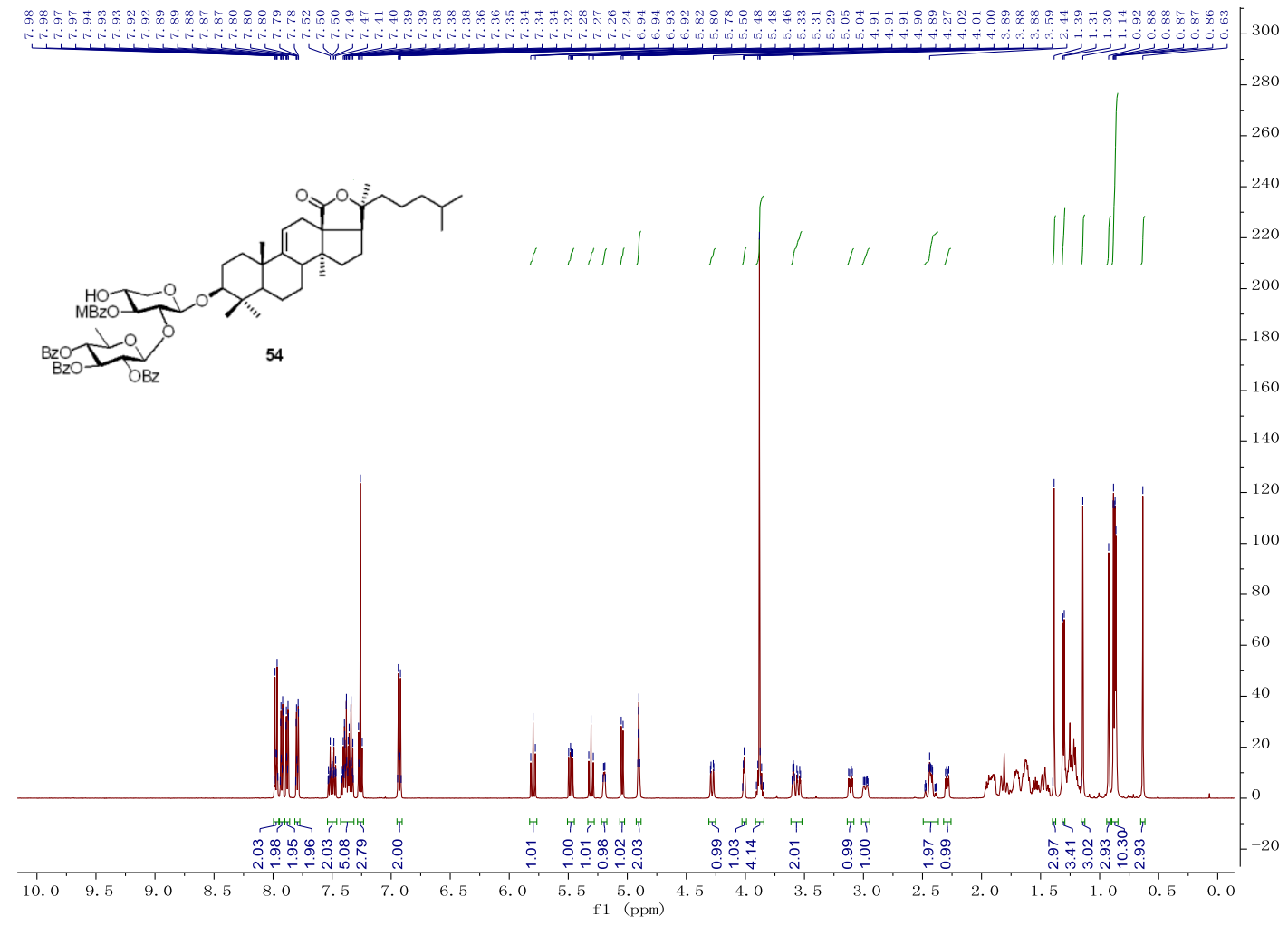

Figure S101. ${ }^{1} \mathrm{H}$ NMR spectrum of compound $54\left(500 \mathrm{MHz}, \mathrm{CDCl}_{3}\right)$

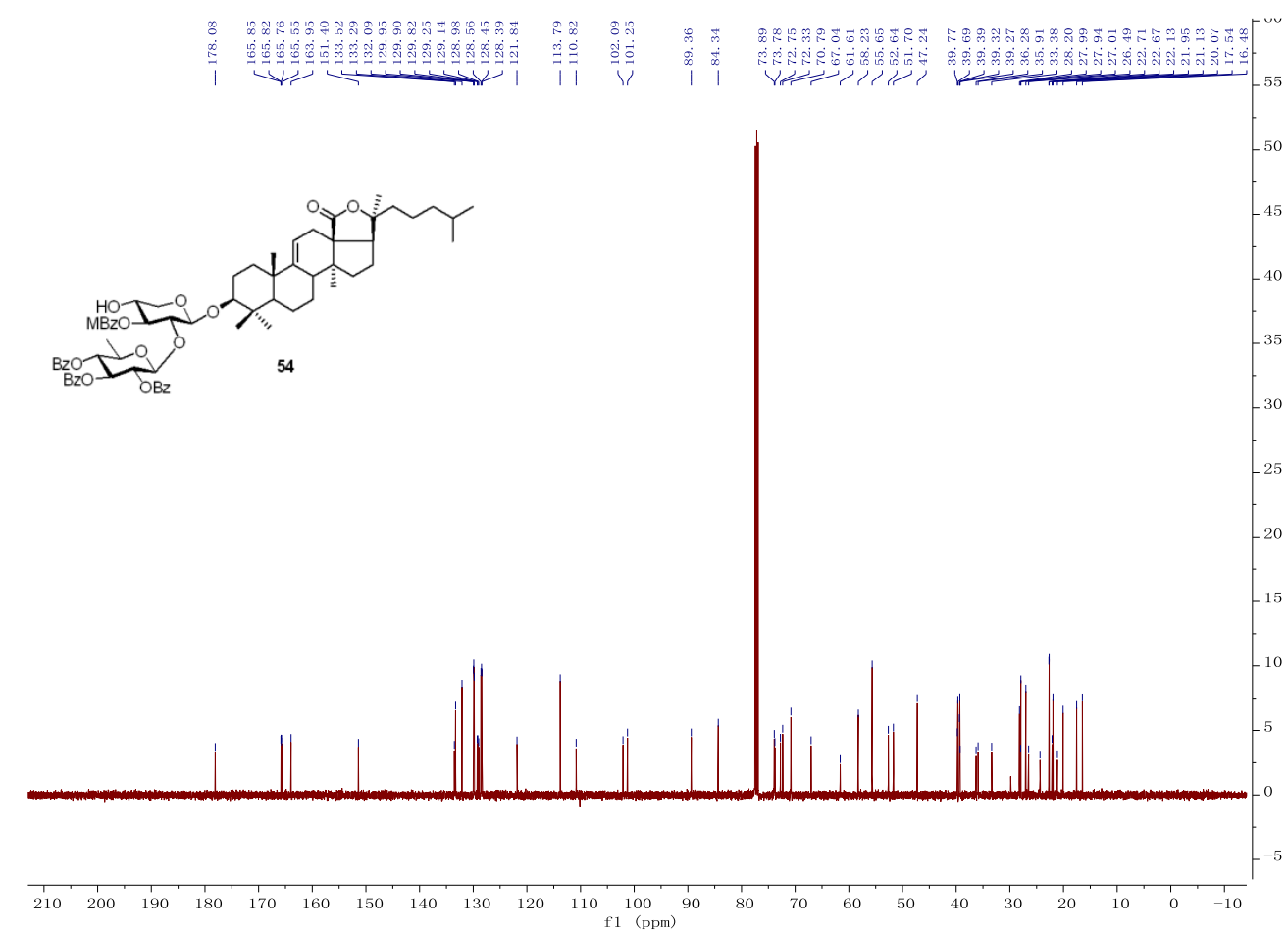

Figure S102. ${ }^{13} \mathrm{C}$ NMR spectrum of compound $54\left(125 \mathrm{MHz}, \mathrm{CDCl}_{3}\right)$ 


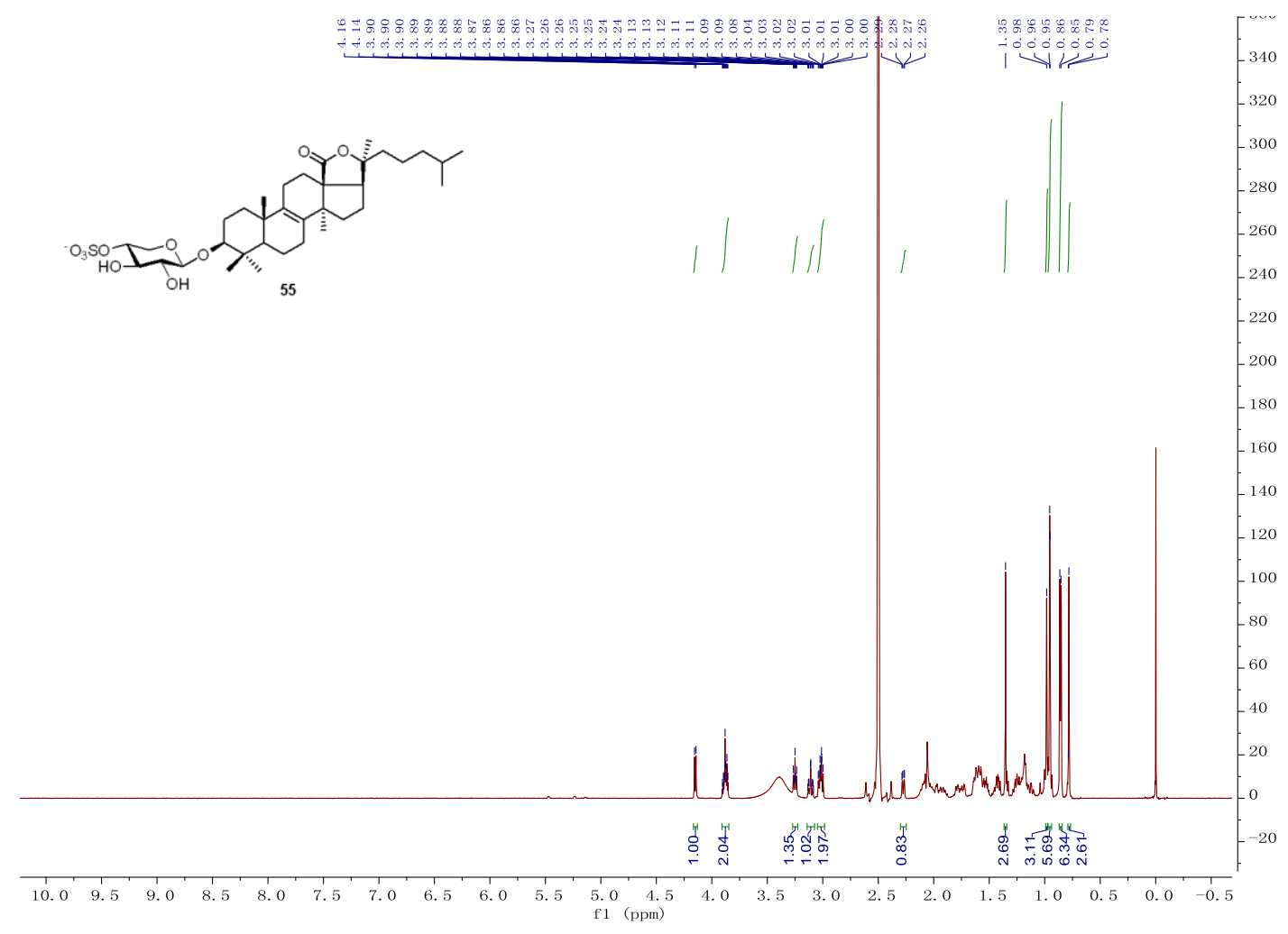

Figure S103. ${ }^{1} \mathrm{H}$ NMR spectrum of compound 55 (600 MHz, DMSO- $d_{6}$ )
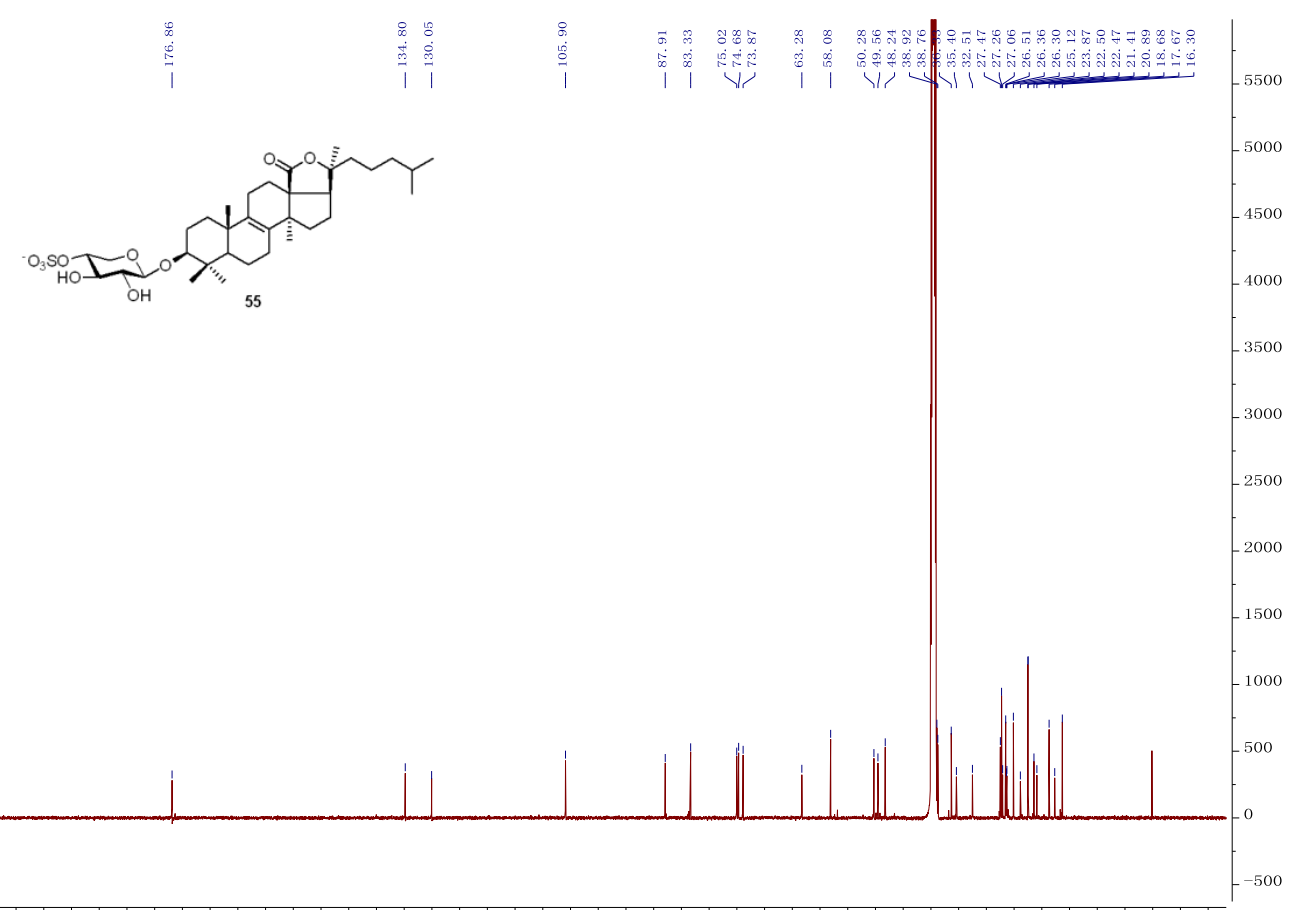

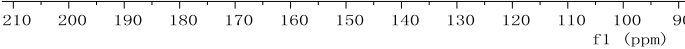

Figure S104. ${ }^{13} \mathrm{C}$ NMR spectrum of compound 55 (150 MHz, DMSO- $d_{6}$ ) 


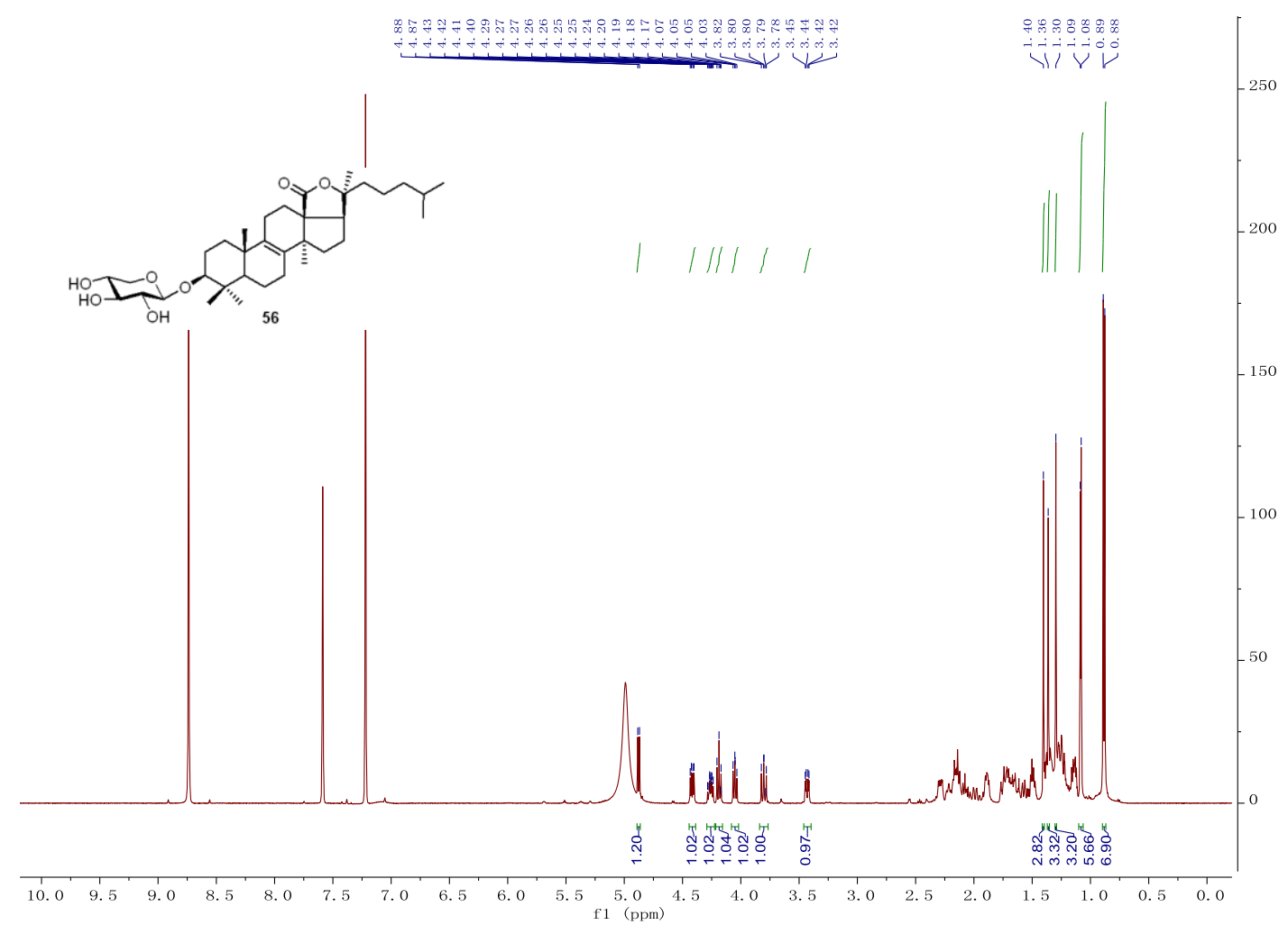

Figure S105. ${ }^{1} \mathrm{H}$ NMR spectrum of compound $56\left(500 \mathrm{MHz}, \mathrm{C}_{5} \mathrm{D}_{5} \mathrm{~N}\right)$

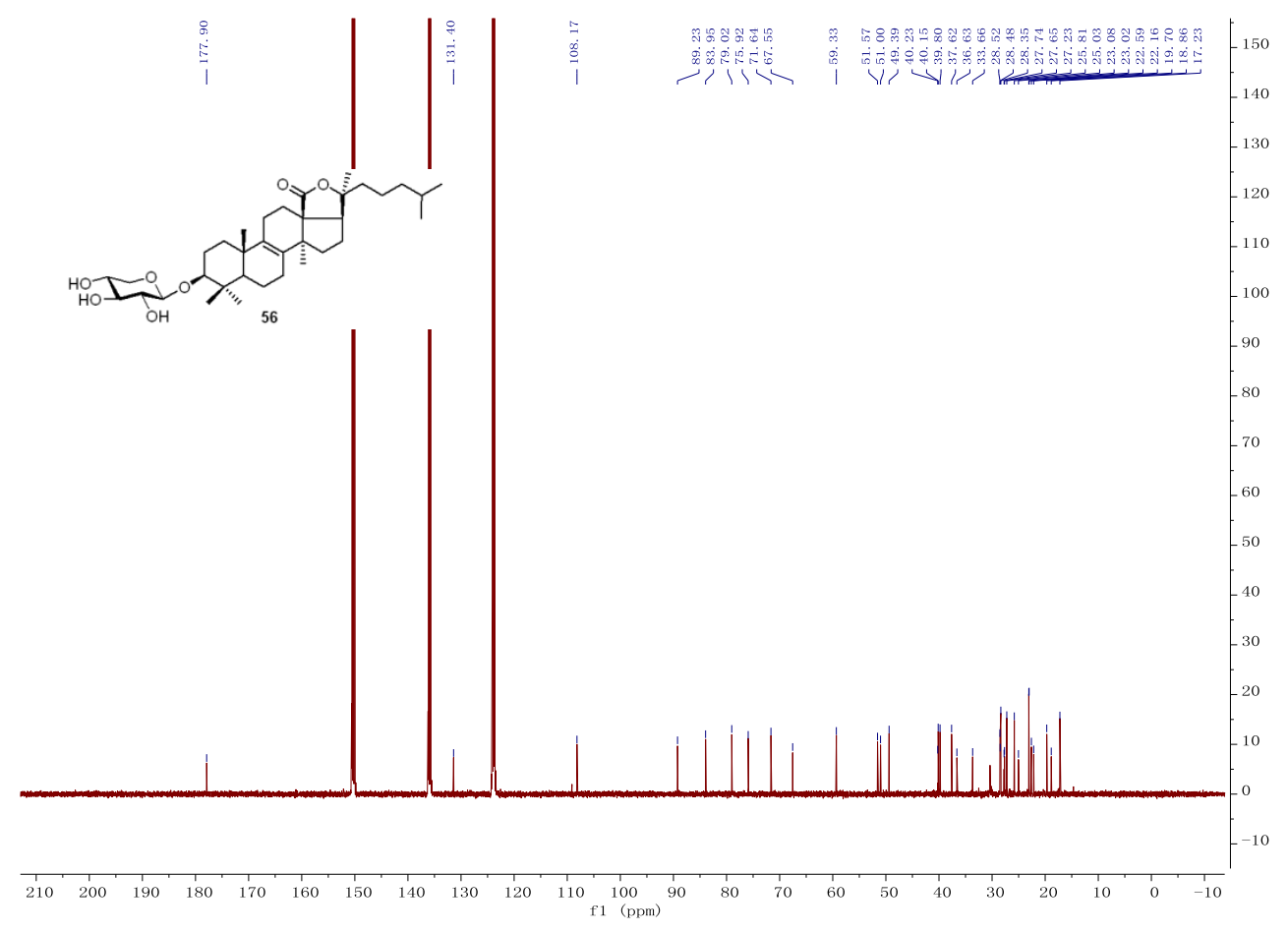

Figure S106. ${ }^{13} \mathrm{C}$ NMR spectrum of compound $56\left(125 \mathrm{MHz}, \mathrm{C}_{5} \mathrm{D}_{5} \mathrm{~N}\right)$ 


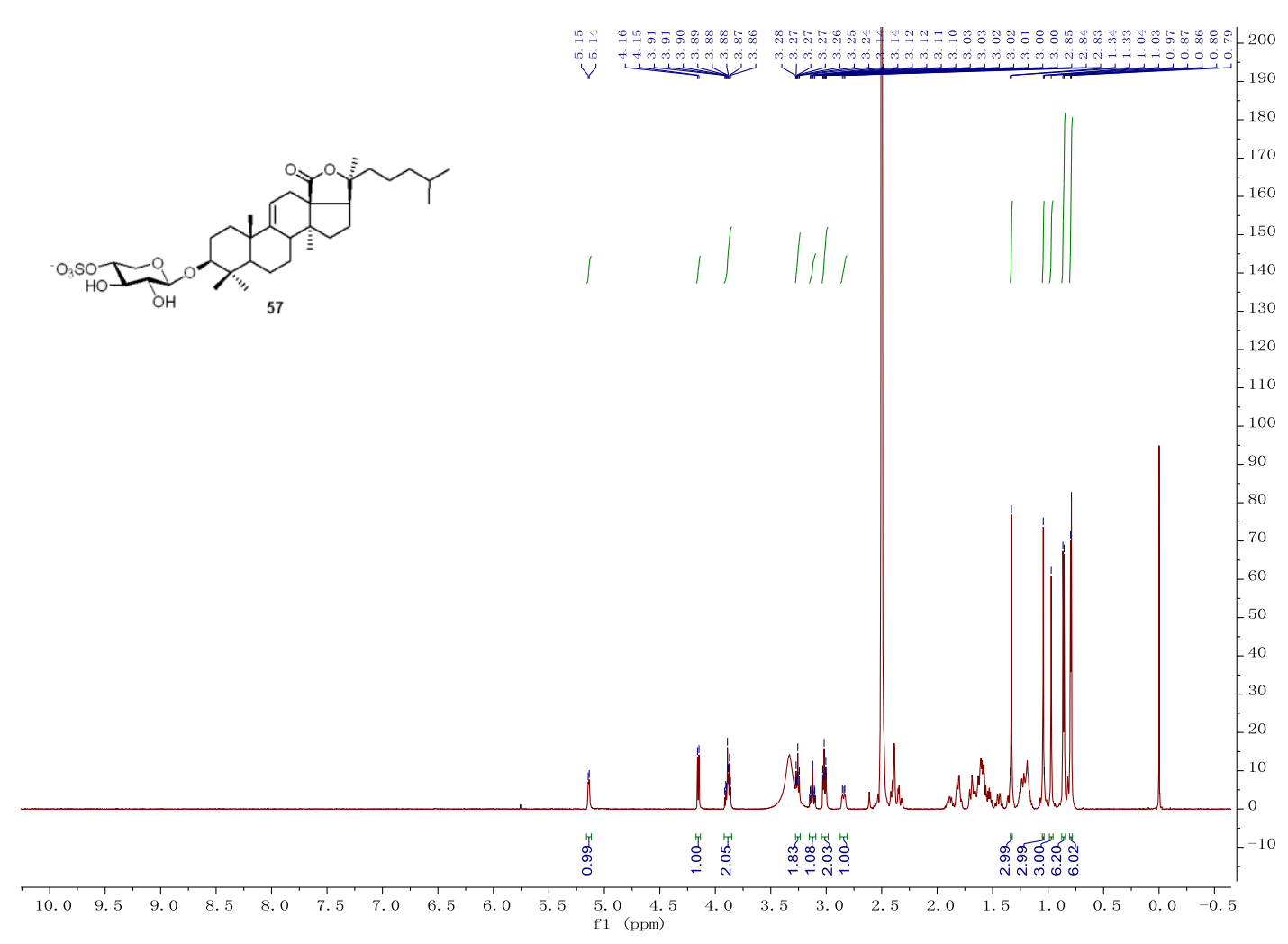

Figure S107. ${ }^{1} \mathrm{H}$ NMR spectrum of compound 57 (600 MHz, DMSO- $d_{6}$ )

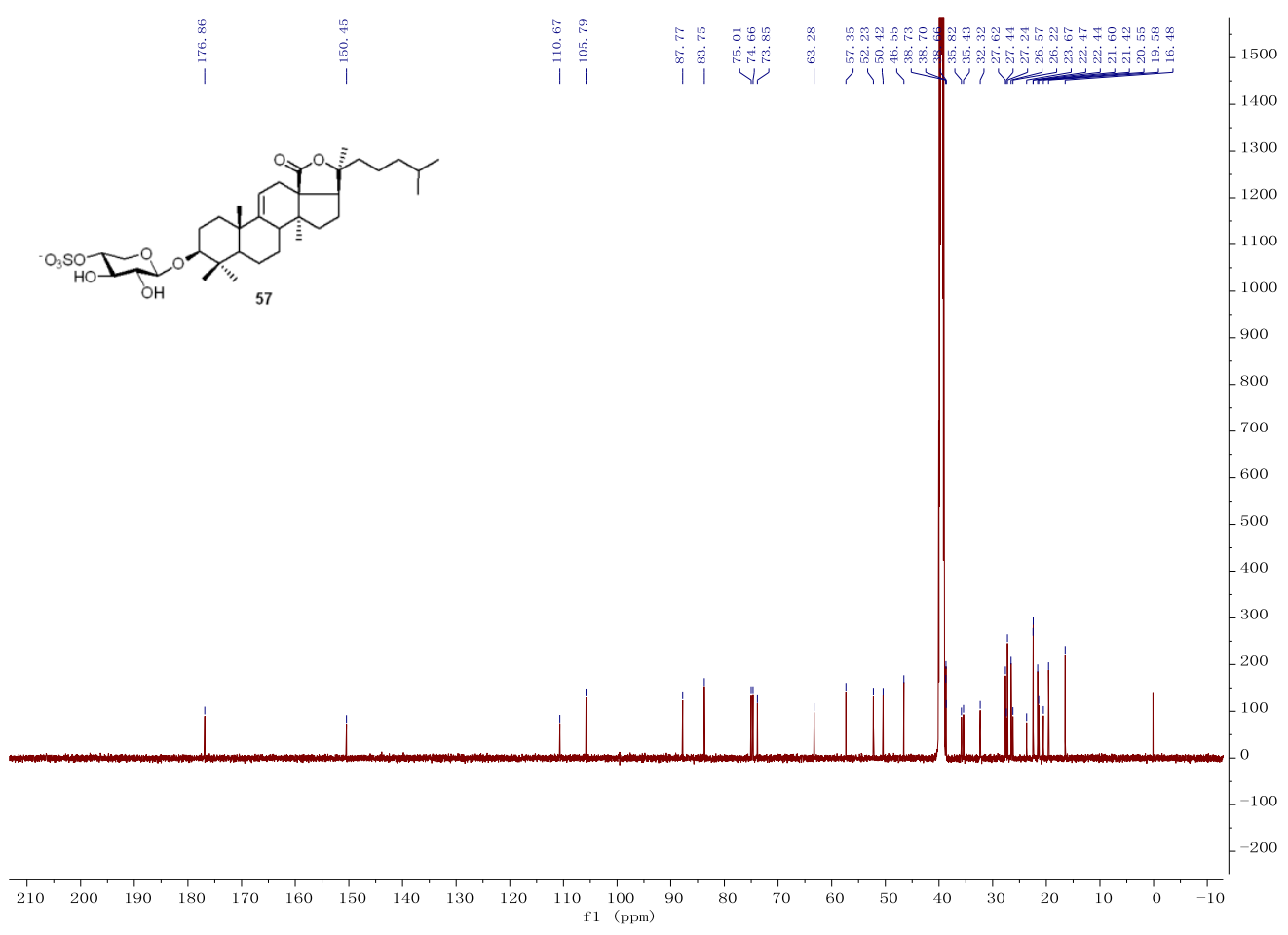

Figure S108. ${ }^{13} \mathrm{C}$ NMR spectrum of compound 57 (150 MHz, DMSO- $d_{6}$ ) 


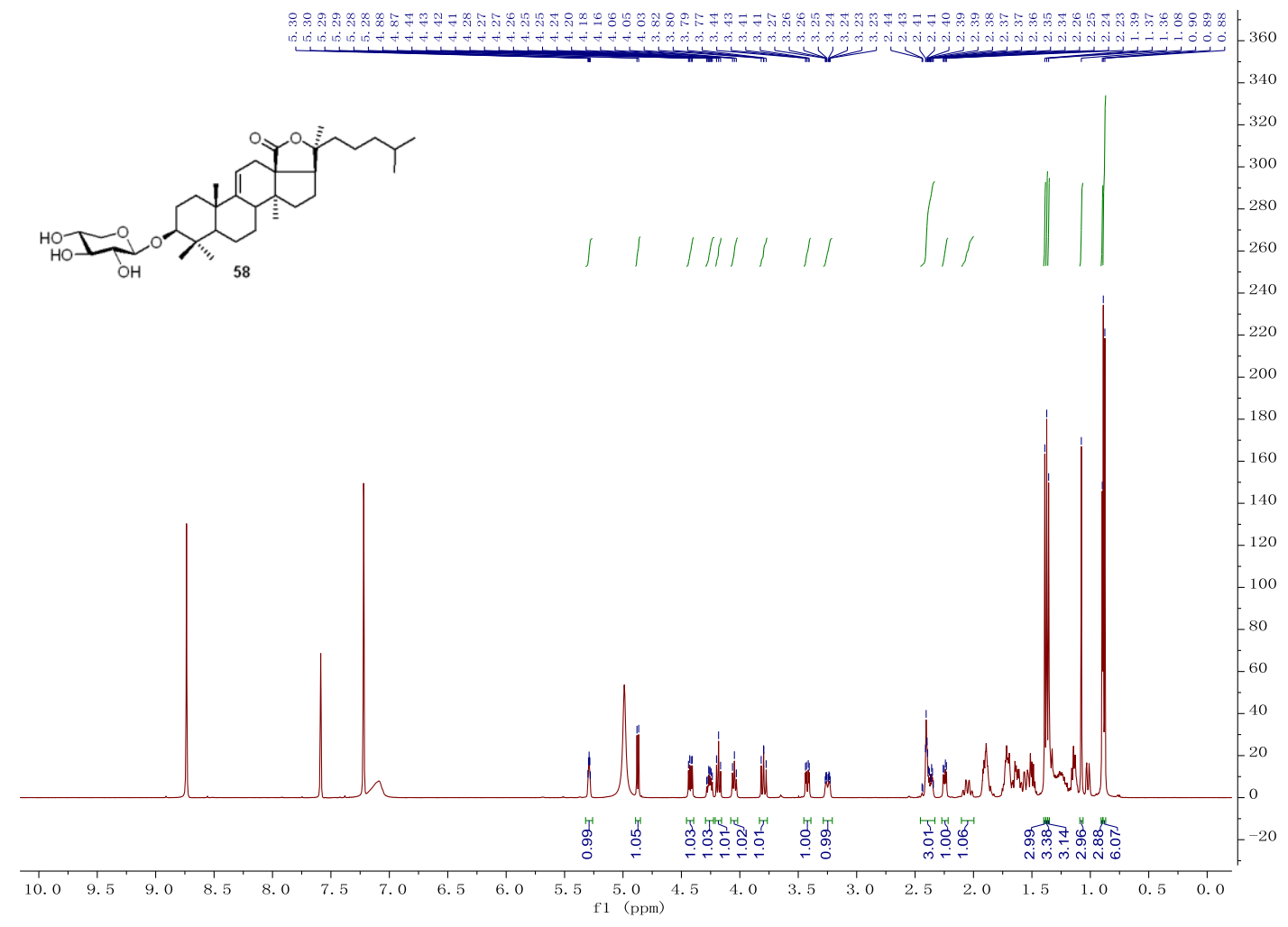

Figure S109. ${ }^{1} \mathrm{H}$ NMR spectrum of compound $\mathbf{5 8}\left(500 \mathrm{MHz}, \mathrm{C}_{5} \mathrm{D}_{5} \mathrm{~N}\right)$

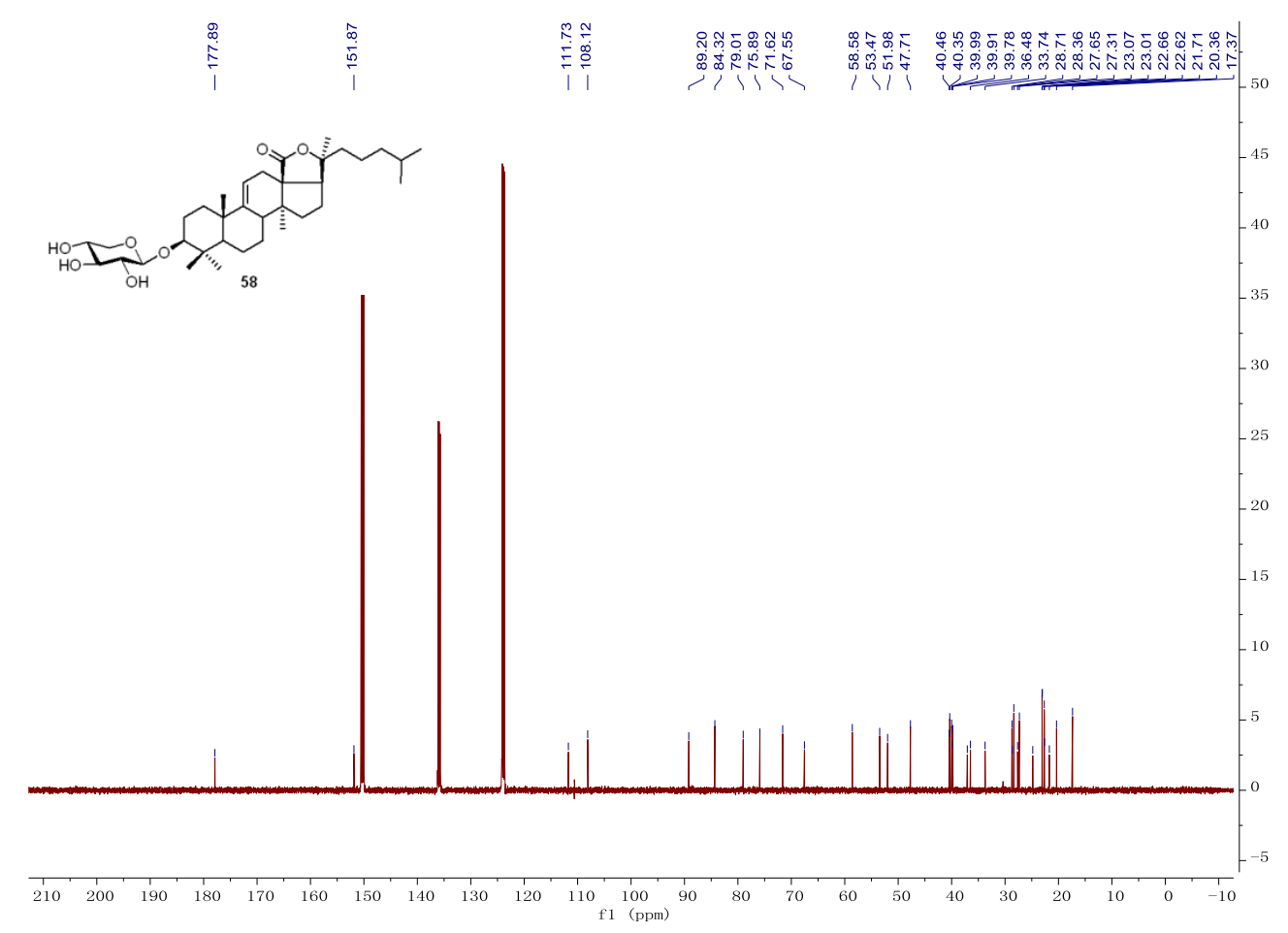

Figure S110. ${ }^{13} \mathrm{C}$ NMR spectrum of compound $58\left(125 \mathrm{MHz}, \mathrm{C}_{5} \mathrm{D}_{5} \mathrm{~N}\right)$ 


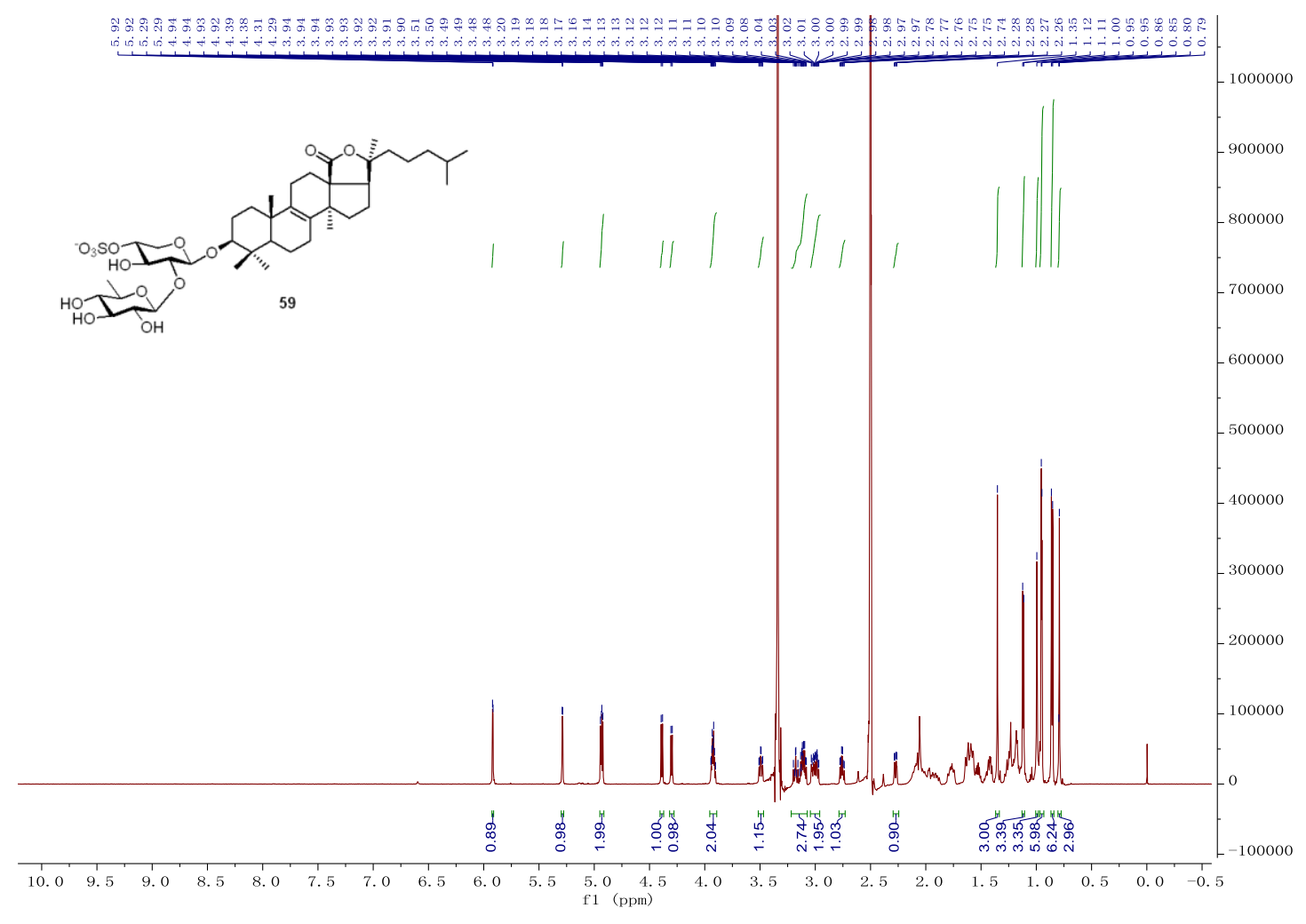

Figure S111. ${ }^{1} \mathrm{H}$ NMR spectrum of compound 59 (600 MHz, DMSO-d6)

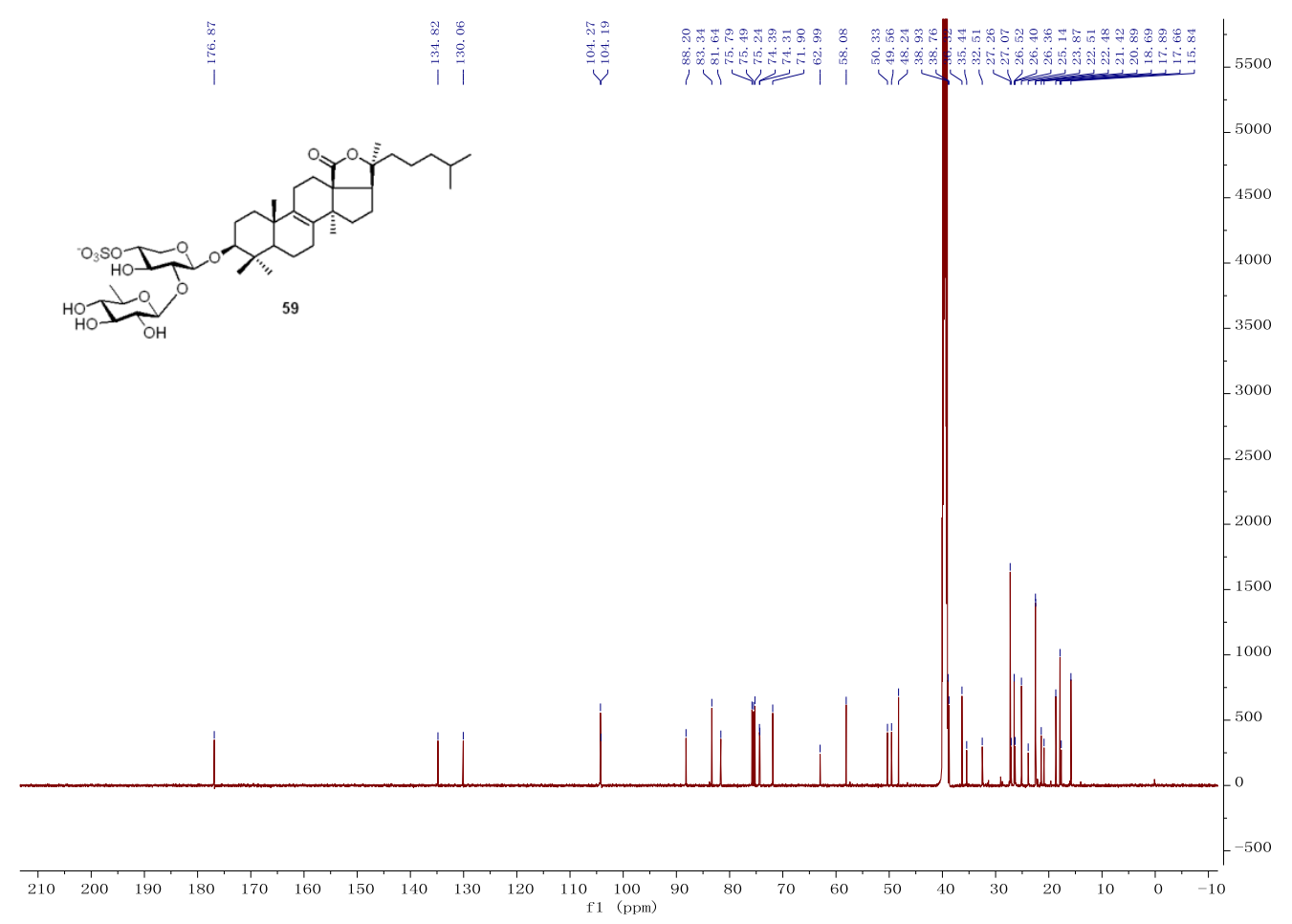

Figure S112. ${ }^{13} \mathrm{C}$ NMR spectrum of compound 59 (150 MHz, DMSO- $d_{6}$ ) 


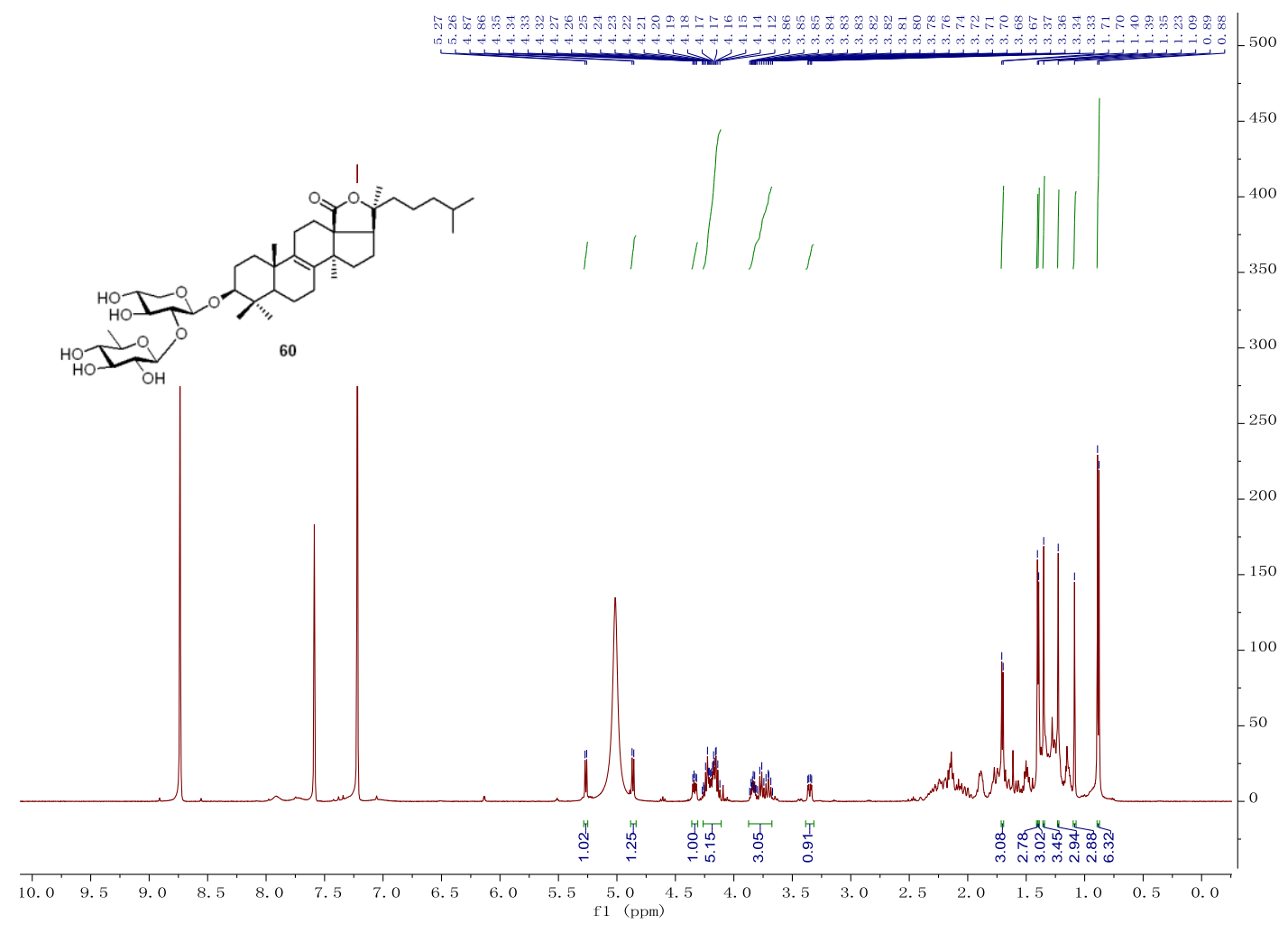

Figure S113. ${ }^{1} \mathrm{H}$ NMR spectrum of compound $60\left(500 \mathrm{MHz}, \mathrm{C}_{5} \mathrm{D}_{5} \mathrm{~N}\right)$

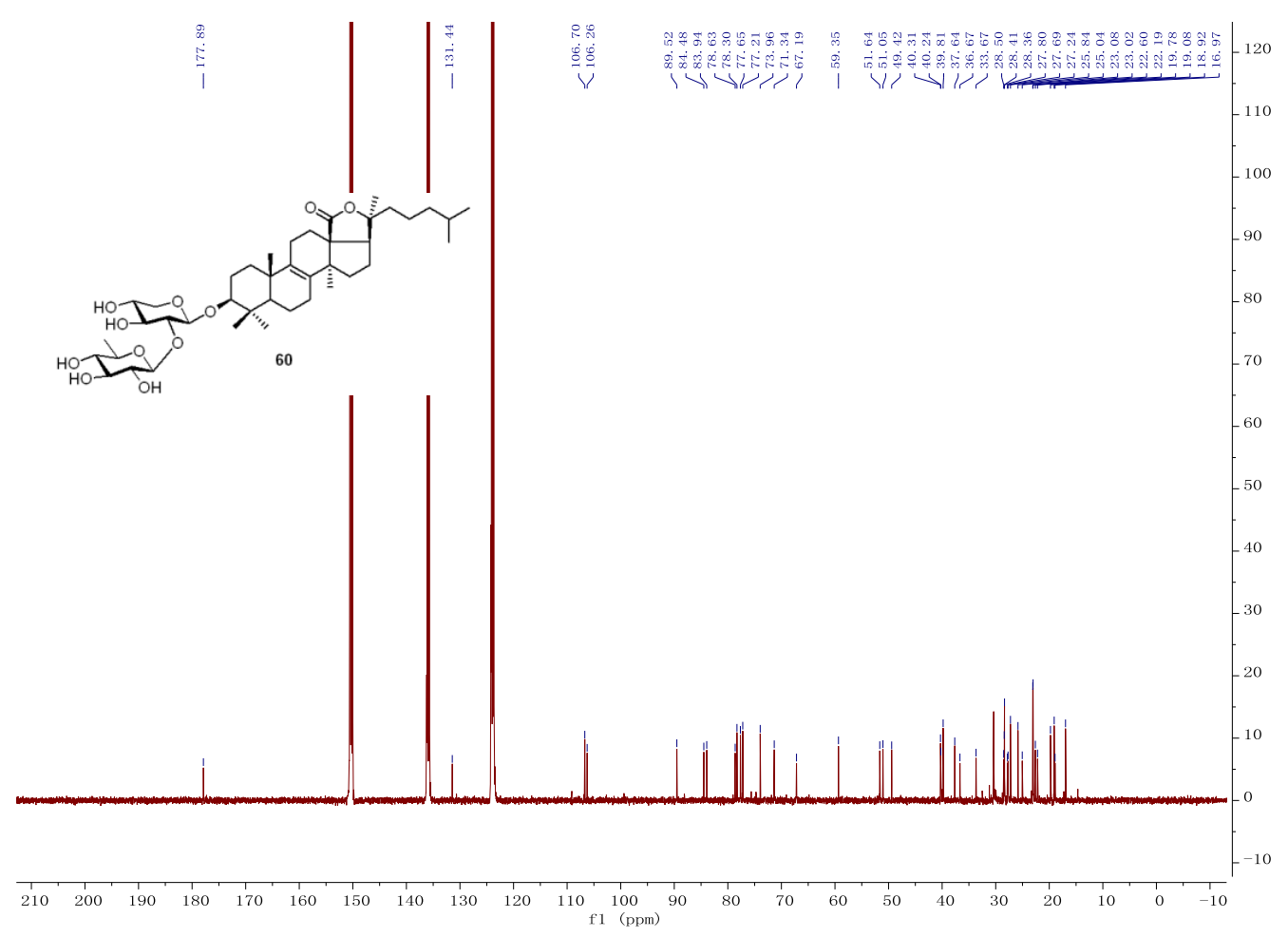

Figure S114. ${ }^{13} \mathrm{C}$ NMR spectrum of compound $60\left(125 \mathrm{MHz}, \mathrm{C}_{5} \mathrm{D}_{5} \mathrm{~N}\right)$ 


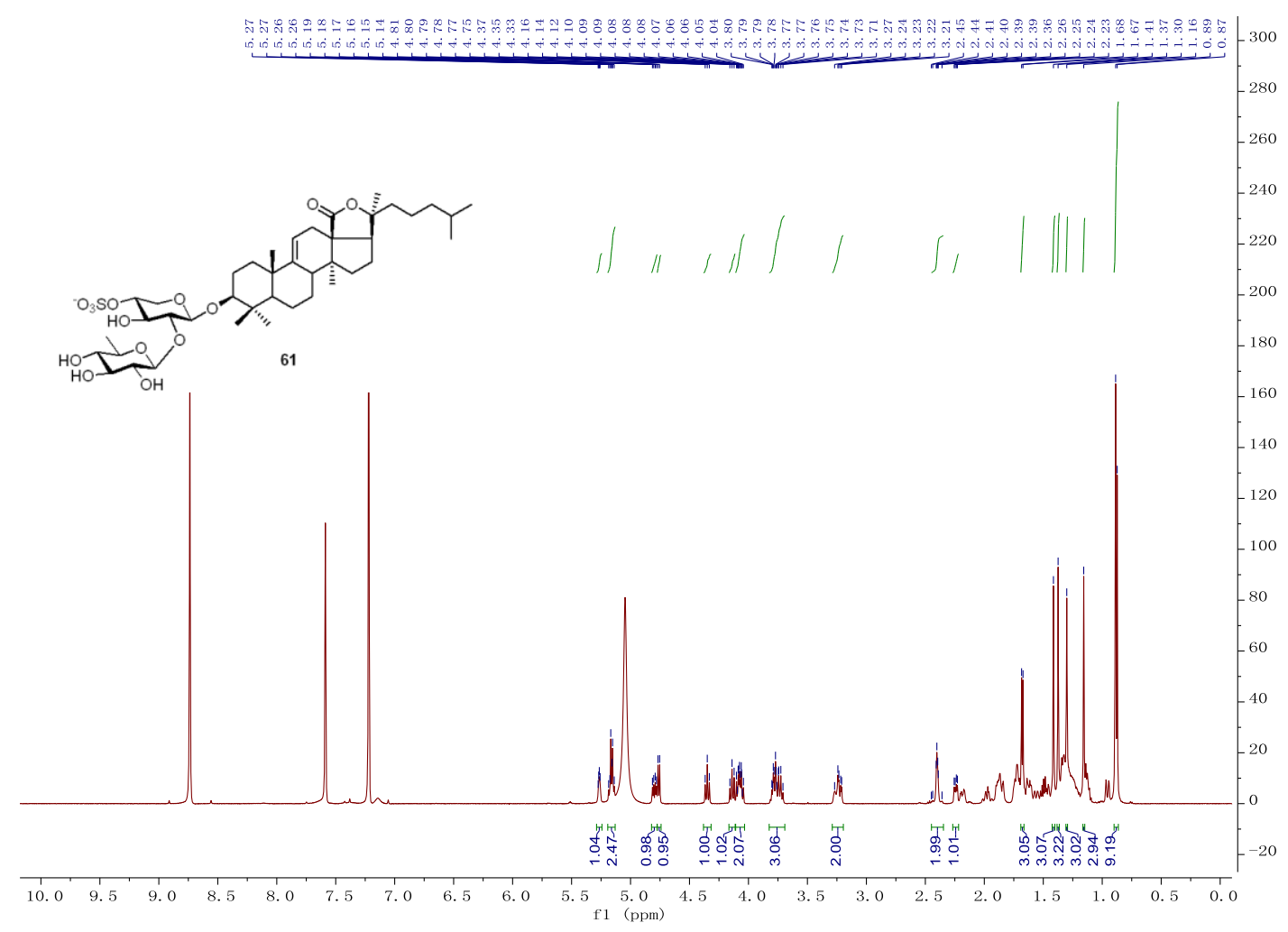

Figure S115. ${ }^{1} \mathrm{H}$ NMR spectrum of compound $61\left(500 \mathrm{MHz}, \mathrm{C}_{5} \mathrm{D}_{5} \mathrm{~N}\right)$

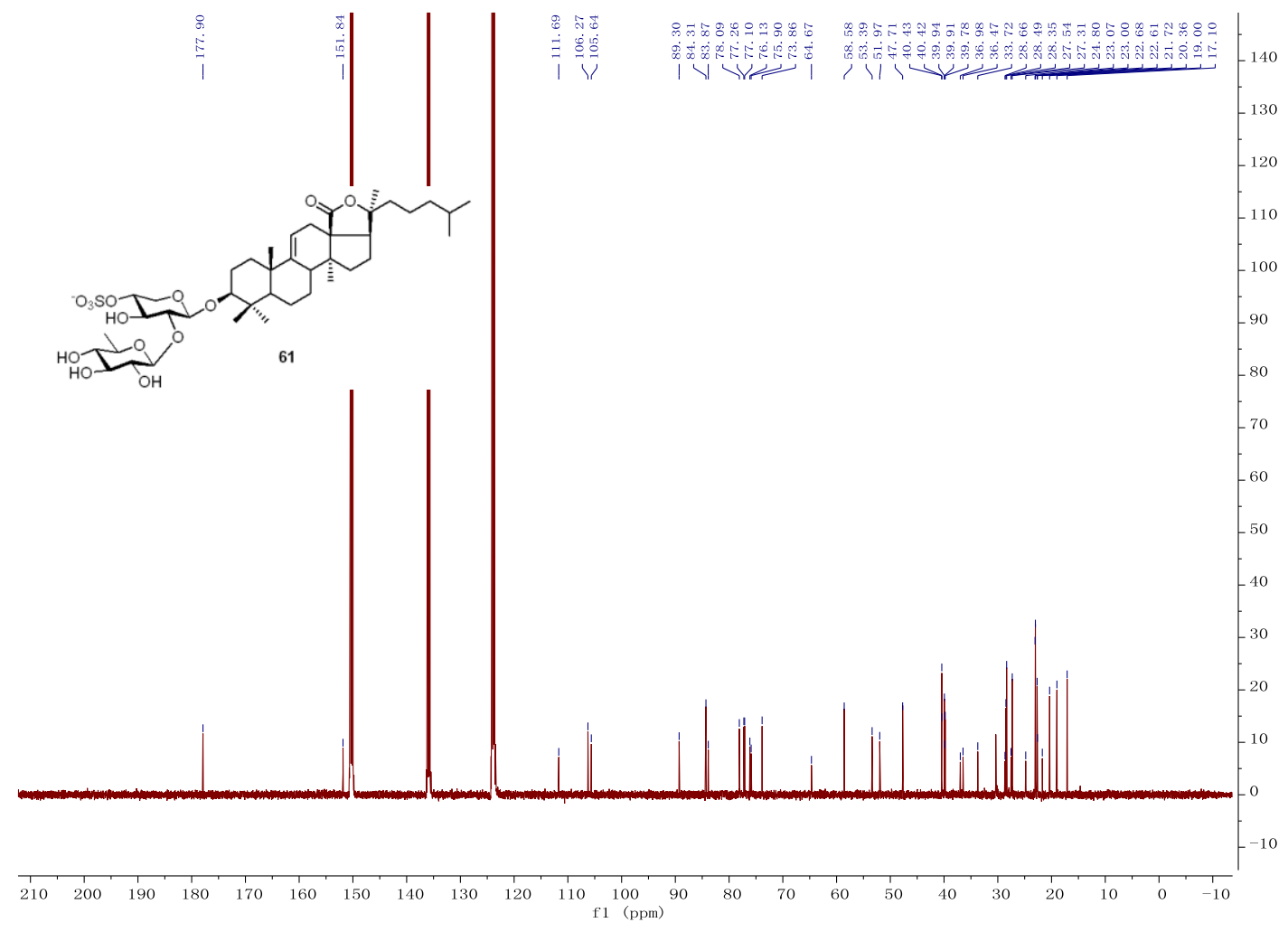

Figure S116. ${ }^{13} \mathrm{C}$ NMR spectrum of compound $61\left(125 \mathrm{MHz}, \mathrm{C}_{5} \mathrm{D}_{5} \mathrm{~N}\right)$ 


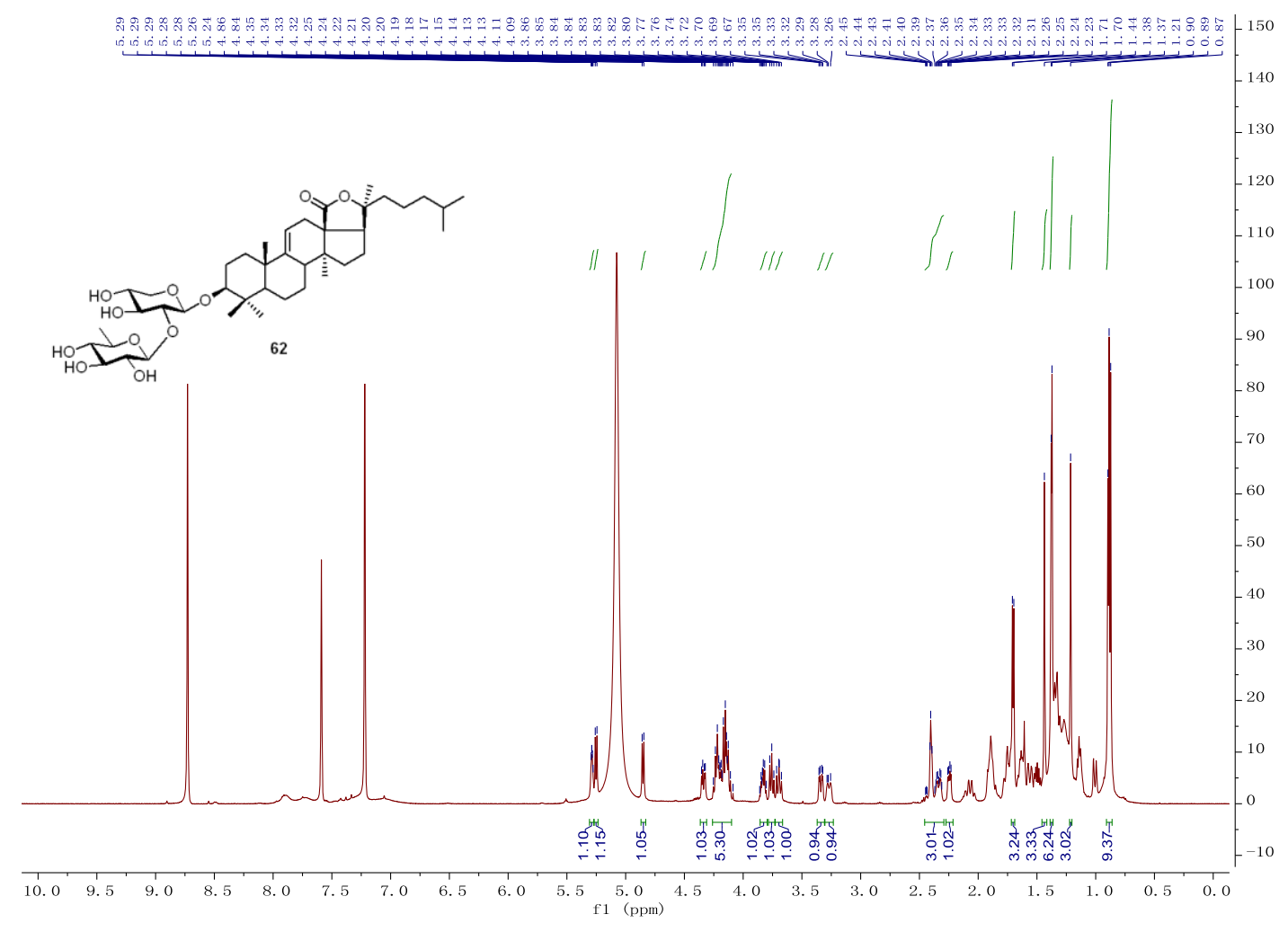

Figure S117. ${ }^{1} \mathrm{H}$ NMR spectrum of compound $62\left(500 \mathrm{MHz}, \mathrm{C}_{5} \mathrm{D}_{5} \mathrm{~N}\right)$

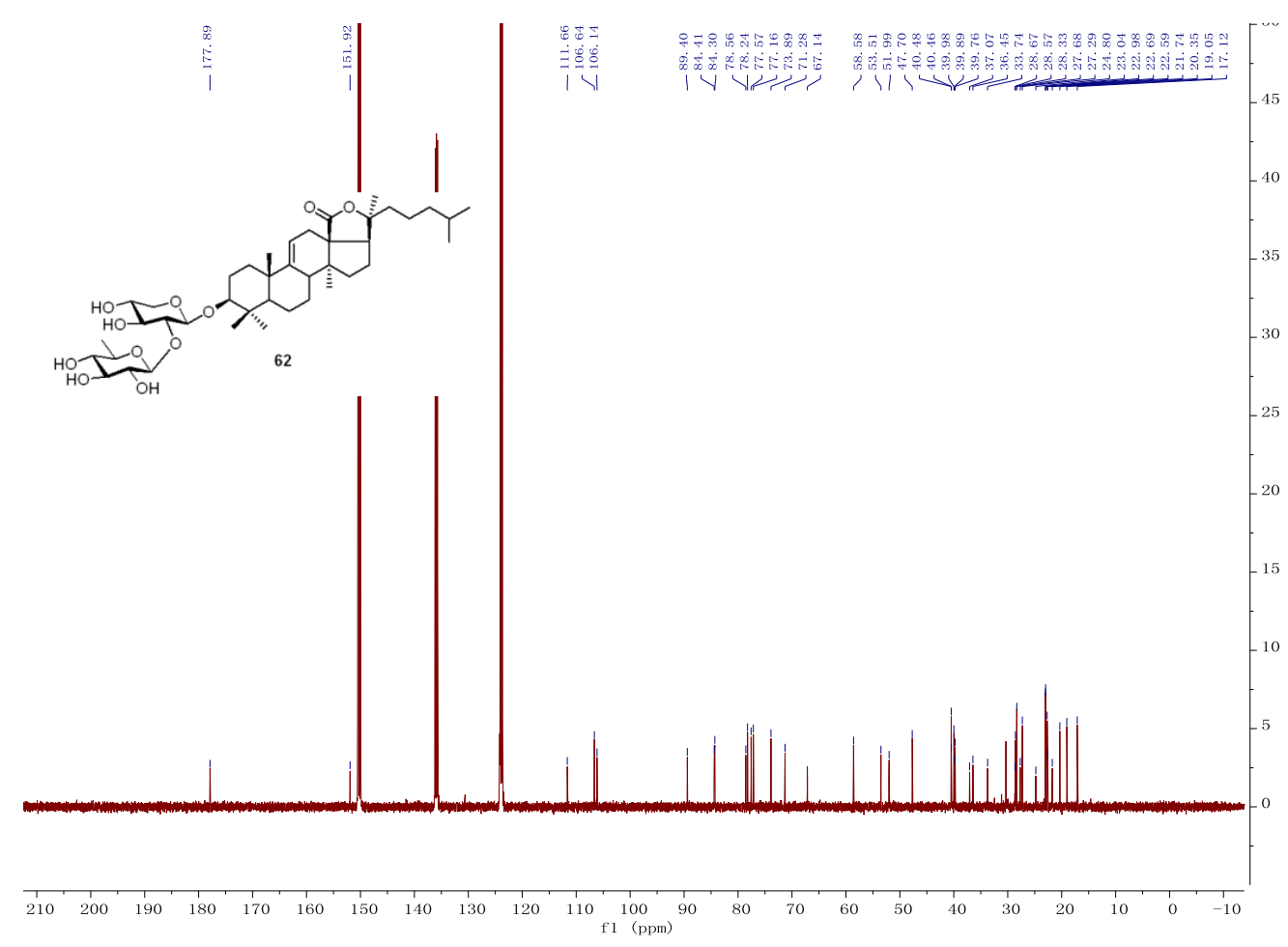

Figure S118. ${ }^{13} \mathrm{C}$ NMR spectrum of compound $62\left(125 \mathrm{MHz}, \mathrm{C}_{5} \mathrm{D}_{5} \mathrm{~N}\right)$ 
HRMS analysis reports

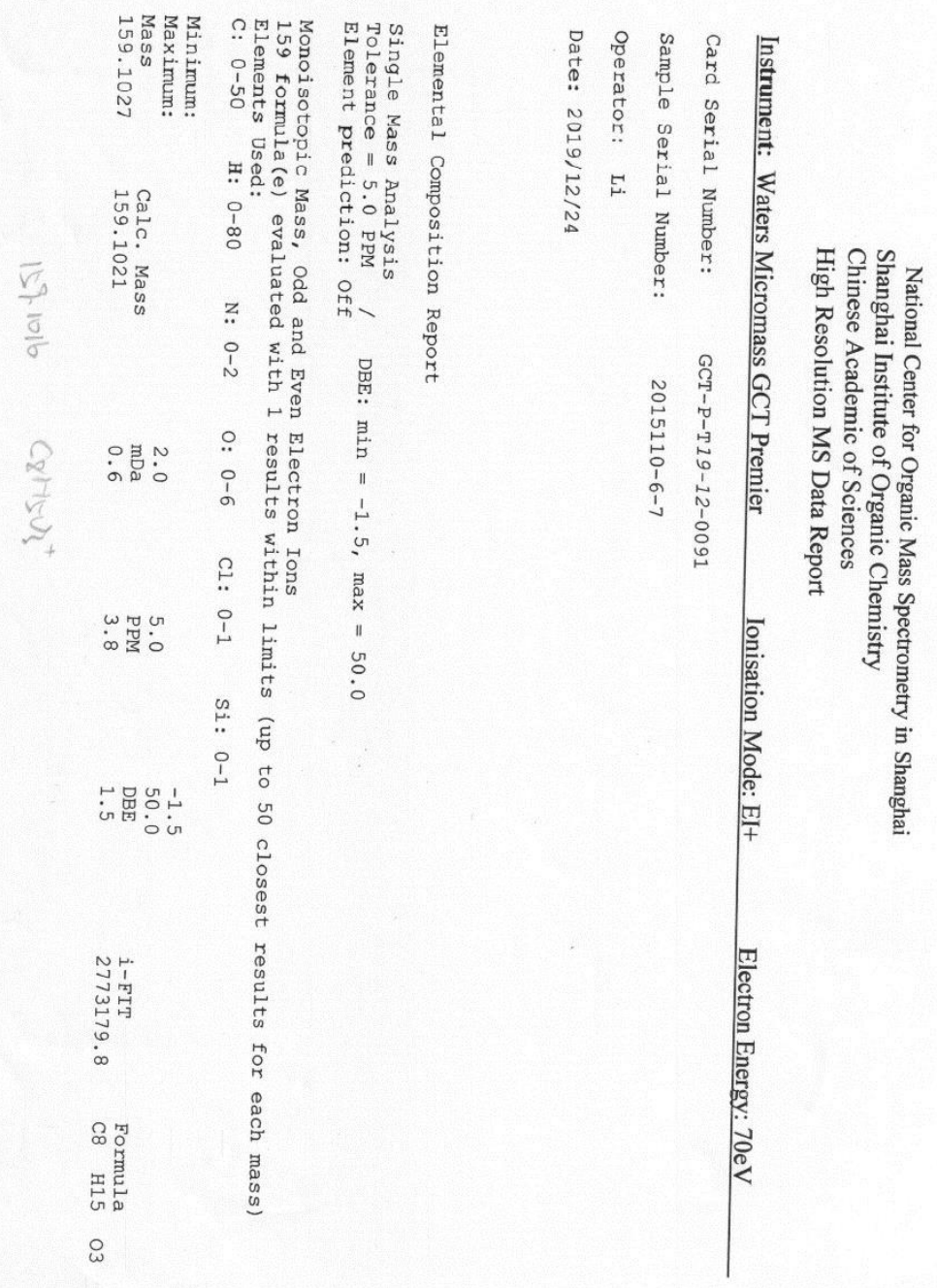

Figure S119. HRMS of compound 6 


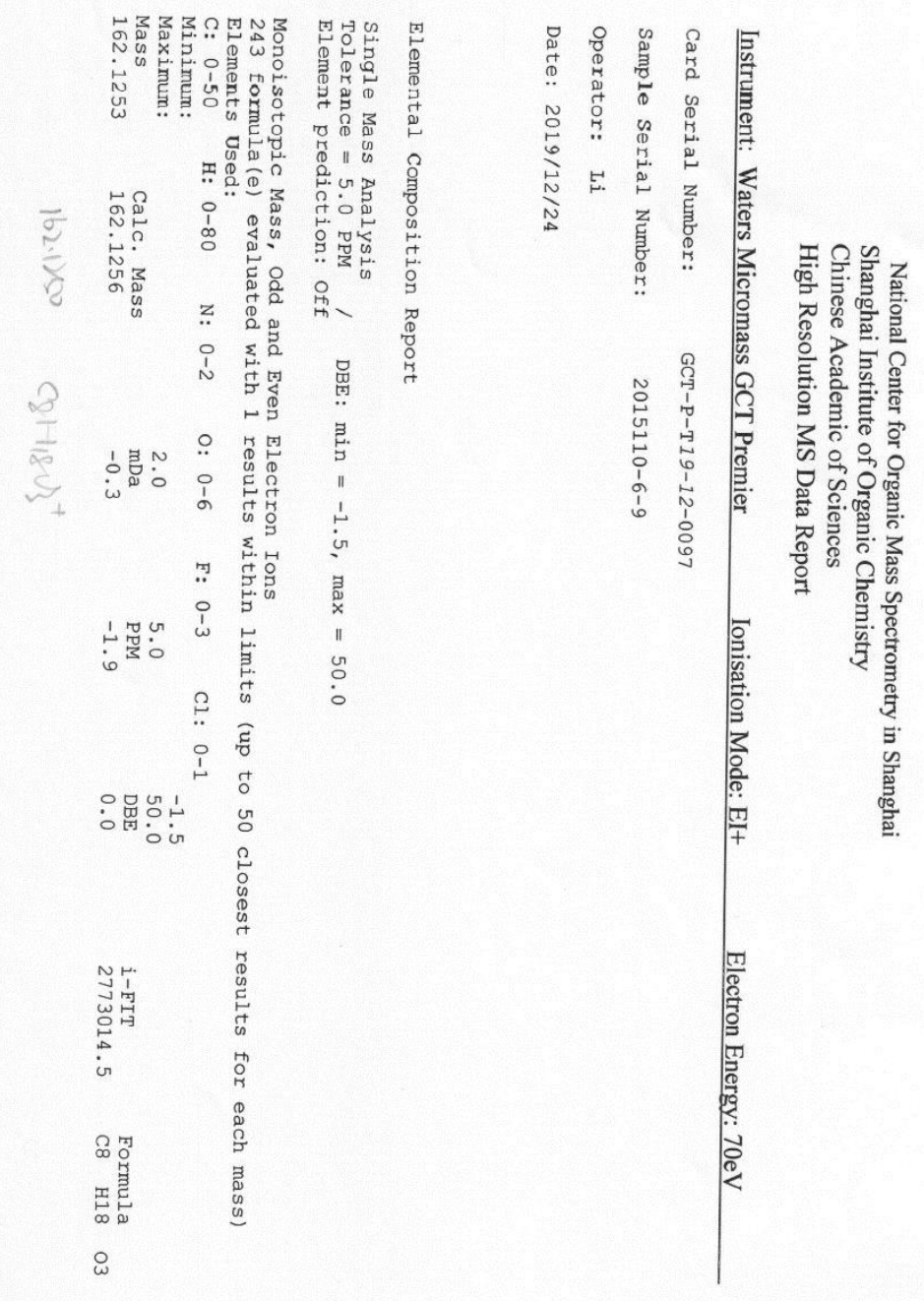

Figure S120. HRMS of compound 7 


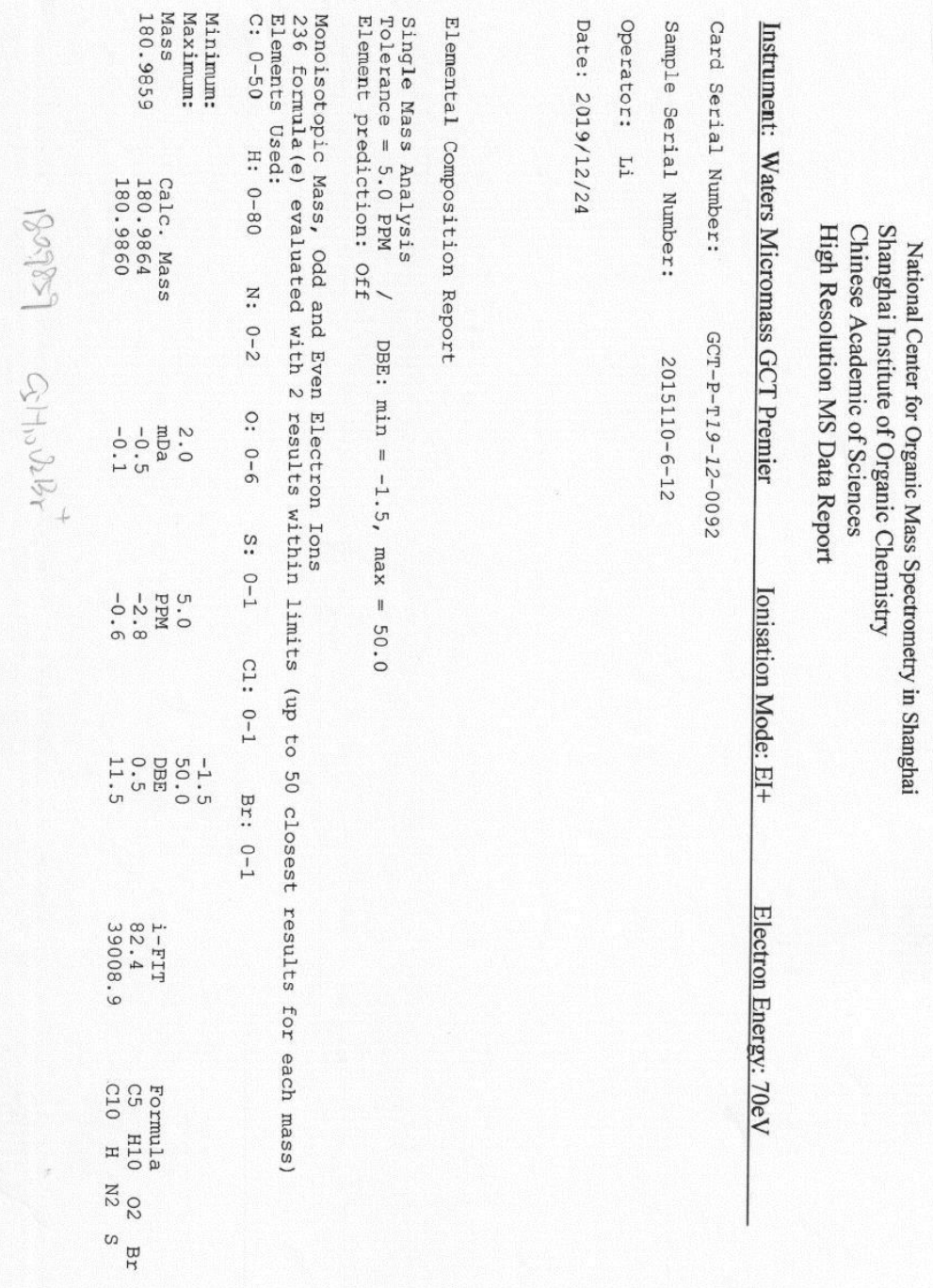

Figure S121. HRMS of compound 8 
National Center for Organic Mass Spectrometry in Shanghai

Shanghai Institute of Organic Chemistry

Chinese Academic of Sciences

High Resolution MS DATA REPORT

Instrument: Thermo Fisher Scientific LTQ FTICR-MS

Card Serial Number : E173489

Sample Serial Number: 5-41-1

Operator: ZHUFJ Date: 2017/08/24

Operation Mode: ESI Postive Ion Mode

Elemental composition search on mass 633.45

$\mathrm{m} / \mathrm{z}=628.45-638.45$

\begin{tabular}{|c|c|c|c|c|}
\hline $\mathrm{m} / \mathrm{z}$ & $\begin{array}{l}\text { Theo. } \\
\text { Mass }\end{array}$ & $\begin{array}{r}\text { Delta } \\
\text { (ppm) }\end{array}$ & $\begin{array}{l}\text { RDB } \\
\text { equiv. }\end{array}$ & Composition \\
\hline 487 & 633.4489 & -0.39 & 8.5 & $\mathrm{C}_{39 \mathrm{H}} 62 \mathrm{O}_{5} \mathrm{Na}$ \\
\hline & 633.4476 & 1.73 & 9.0 & $\mathrm{C}_{37} \mathrm{H}_{60} \mathrm{O}_{4} \mathrm{~N}_{3} \mathrm{Na}$ \\
\hline & 633.4500 & -2.07 & 12.0 & $\mathrm{C}_{39} \mathrm{H}_{59} \mathrm{O}_{4} \mathrm{~N}_{3}$ \\
\hline & 633.4473 & 2.16 & 7.5 & $\mathrm{C}_{36} \mathrm{H}_{61} \mathrm{O}_{7} \mathrm{~N}_{2}$ \\
\hline & 633.4514 & -4.19 & 11.5 & $\mathrm{C}_{41} \mathrm{H}_{61} \mathrm{O}_{5}$ \\
\hline & 633.451 & -4.62 & 13.0 & $\mathrm{C}_{42} \mathrm{H}_{60} \mathrm{O}_{2} \mathrm{~N} \mathrm{Na}$ \\
\hline
\end{tabular}

Figure S122. HRMS of compound 10 
National Center for Organic Mass Spectrometry in Shanghai Shanghai Institute of Organic Chemistry

Chinese Academic of Sciences

High Resolution MS DATA REPORT

Instrument: Thermo Fisher Scientific LTQ FTICR-MS

Card Serial Number : E173488

Sample Serial Number: 5-43-1

Operator : ZHUFJ Date: 2017/08/24

Operation Mode: ESI Postive Ion Mode

Elemental composition search on mass 629.42

$\mathrm{m} / \mathrm{z}=624.42-634.42$

\begin{tabular}{c|c|c|c|c}
$\mathrm{m} / \mathrm{z}$ & $\begin{array}{c}\text { Theo. } \\
\text { Mass }\end{array}$ & $\begin{array}{c}\text { Delta } \\
(\mathrm{ppm})\end{array}$ & $\begin{array}{c}\text { RDB } \\
\text { equiv. }\end{array}$ & Composition
\end{tabular}

$629.4175 \quad 629.4176 \quad-0.25 \quad 0.50$

C $39 \mathrm{H}_{58} \mathrm{O}_{5} \mathrm{Na}$

$629.4187 \quad 1.89 \quad 11.0 \mathrm{C}_{37} \mathrm{H}_{56} \mathrm{O}_{4} \mathrm{~N}_{3} \mathrm{Na}$

$2.32-9.5 \mathrm{C}_{36} \mathrm{H}_{57} \mathrm{O}_{7} \mathrm{~N}_{2}$

$\begin{array}{lll}.4203 & -4.51 & 15.0 \mathrm{C}_{42} \mathrm{H}_{56} \mathrm{O}_{2} \mathrm{~N} \mathrm{Na}\end{array}$

Figure S123. HRMS of compound 11 
National Center for Organic Mass Spectrometry in Shanghai

Shanghai Institute of Organic Chemistry

Chinese Academic of Sciences

High Resolution MS DATA REPORT

Instrument: Thermo Fisher Scientific LTQ FTICR-MS

Card Serial Number : E173487

Sample Serial Number: 5-44

Operator : ZHUFJ Date: 2017/08/24

Operation Mode: ESI Postive Ion Mode

Elemental composition search on mass 643.40

$\mathrm{m} / \mathrm{z}=638.40-648.40$

\begin{tabular}{c|c|c|c|c}
$\mathrm{m} / \mathrm{z}$ & $\begin{array}{c}\text { Theo. } \\
\text { Mass }\end{array}$ & $\begin{array}{c}\text { Delta } \\
\text { (ppm) }\end{array}$ & RDB & Composition
\end{tabular}

$643.3969 \quad 643.3969$

$11.5 \mathrm{C}_{39} \mathrm{H}_{56} \mathrm{O}_{6} \mathrm{Na}$

$-1.68 \quad 15.0 \mathrm{C}_{39} \mathrm{H}_{53} \mathrm{O}_{5} \mathrm{~N}_{3}$

$\begin{array}{llll}643.3953 & 2.48 & 12.0 \mathrm{C}_{37} \mathrm{H}_{54} \mathrm{O}_{5} \mathrm{~N}_{3} \mathrm{Na}\end{array}$

$643.3993=-3.77$

$\begin{array}{lll}643.3996-4.20 & 16.0 \mathrm{C}_{42} \mathrm{H}_{54} \mathrm{O}_{3} \mathrm{NNa}\end{array}$

Figure S124. HRMS of compound 12 
National Center for Organic Mass Spectrometry in Shanghai

Shanghai Institute of Organic Chemistry

Chinese Academic of Sciences

High Resolution MS DATA REPORT

Instrument: Thermo Fisher Scientific LTQ FTICR-MS

Card Serial Number : E173490

Sample Serial Number: 2015110-5-51

Operator : ZHUFJ Date: $2017 / 08 / 24$

Operation Mode: ESI Postive Ion Mode

Elemental composition search on mass 629.42

$\mathrm{m} / \mathrm{z}=624.42-634.42$

\begin{tabular}{|c|c|c|c|c|}
\hline $\mathrm{m} / \mathrm{z}$ & $\begin{array}{r}\text { Theo. } \\
\text { Mass }\end{array}$ & $\begin{array}{r}\text { Delta } \\
\text { (ppm) }\end{array}$ & $\begin{array}{l}\text { RDB } \\
\text { equiv. }\end{array}$ & Compositi \\
\hline 29.4175 & 629.4176 & -0.25 & 10.5 & $\mathrm{C}_{39} \mathrm{H}_{58} \mathrm{O}_{5} \mathrm{Na}$ \\
\hline & 629.4163 & 1.89 & 11.0 & $\mathrm{C}_{37} \mathrm{H}_{56} \mathrm{O}_{4} \mathrm{~N}_{3} \mathrm{Na}$ \\
\hline & 629.4187 & -1.94 & 14.0 & $\mathrm{C}_{39} \mathrm{H}_{55} \mathrm{O}_{4} \mathrm{~N}_{3}$ \\
\hline & 629.4160 & 2.32 & 9.5 & $\mathrm{C}_{36} \mathrm{H}_{57} \mathrm{O}_{7} \mathrm{~N}_{2}$ \\
\hline & 629.4201 & -4.07 & 13.5 & $\mathrm{C}_{41} \mathrm{H}_{57} \mathrm{O}_{5}$ \\
\hline & 629.420 & -4.51 & 15.0 & $\mathrm{C}_{42} \mathrm{H}_{56} \mathrm{O}_{2} \mathrm{~N} \mathrm{Na}$ \\
\hline
\end{tabular}

Figure S125. HRMS of compound 13 


\section{Mass Spectrum SmartFormula Report}

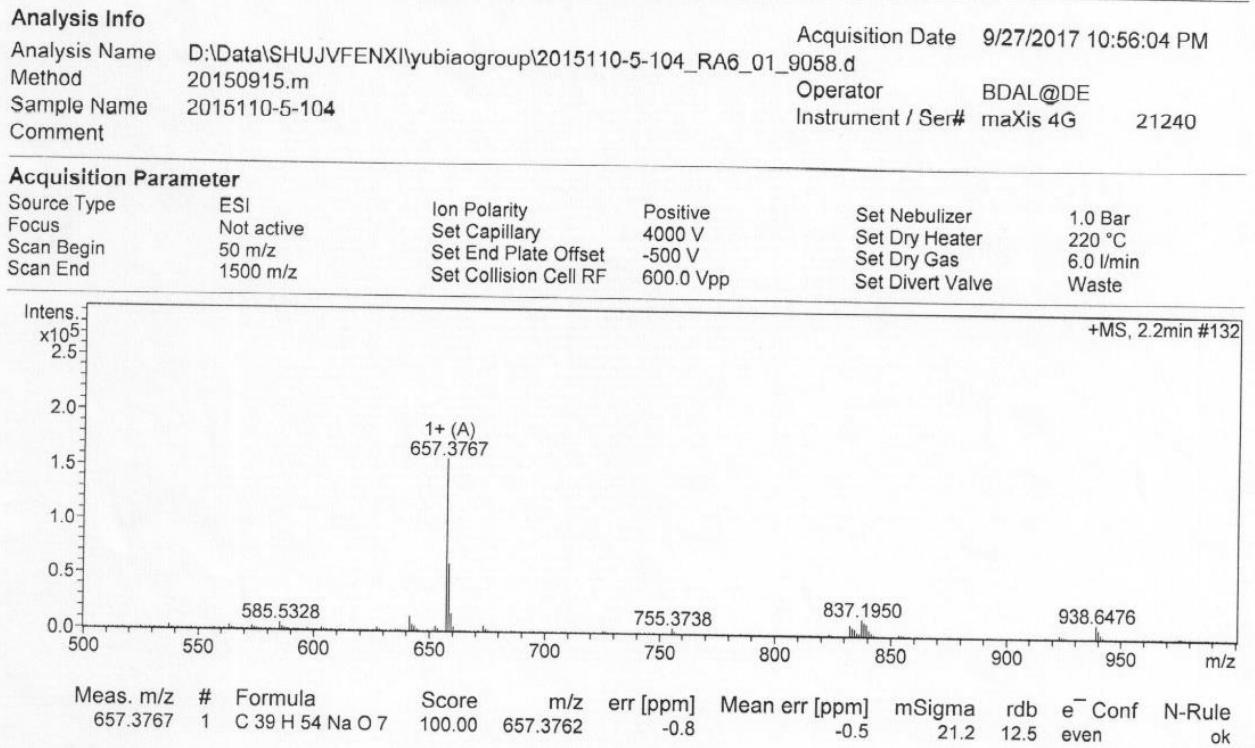

Figure S126. HRMS of compound 14 


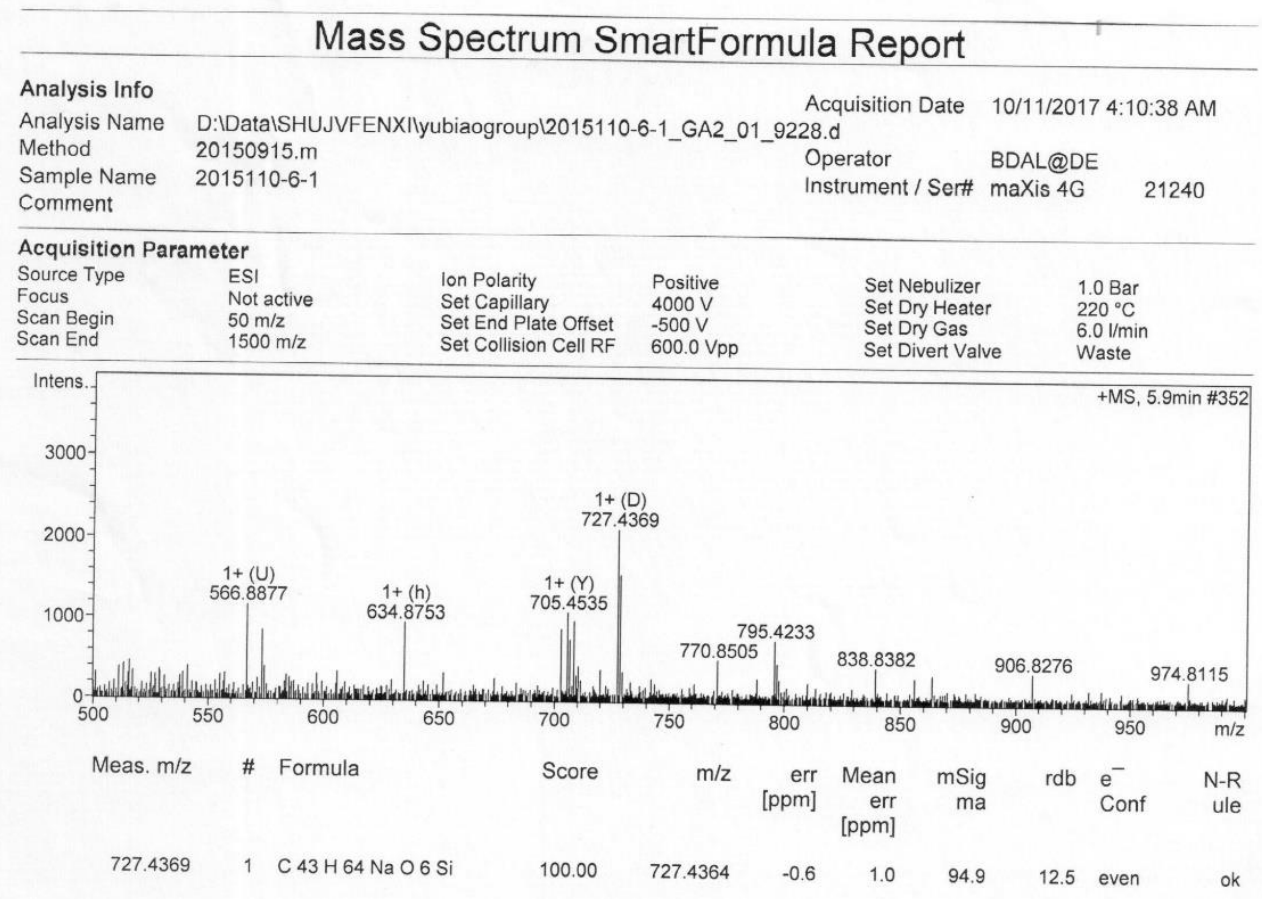

Figure S127. HRMS of compound 15 


\section{Mass Spectrum SmartFormula Report}

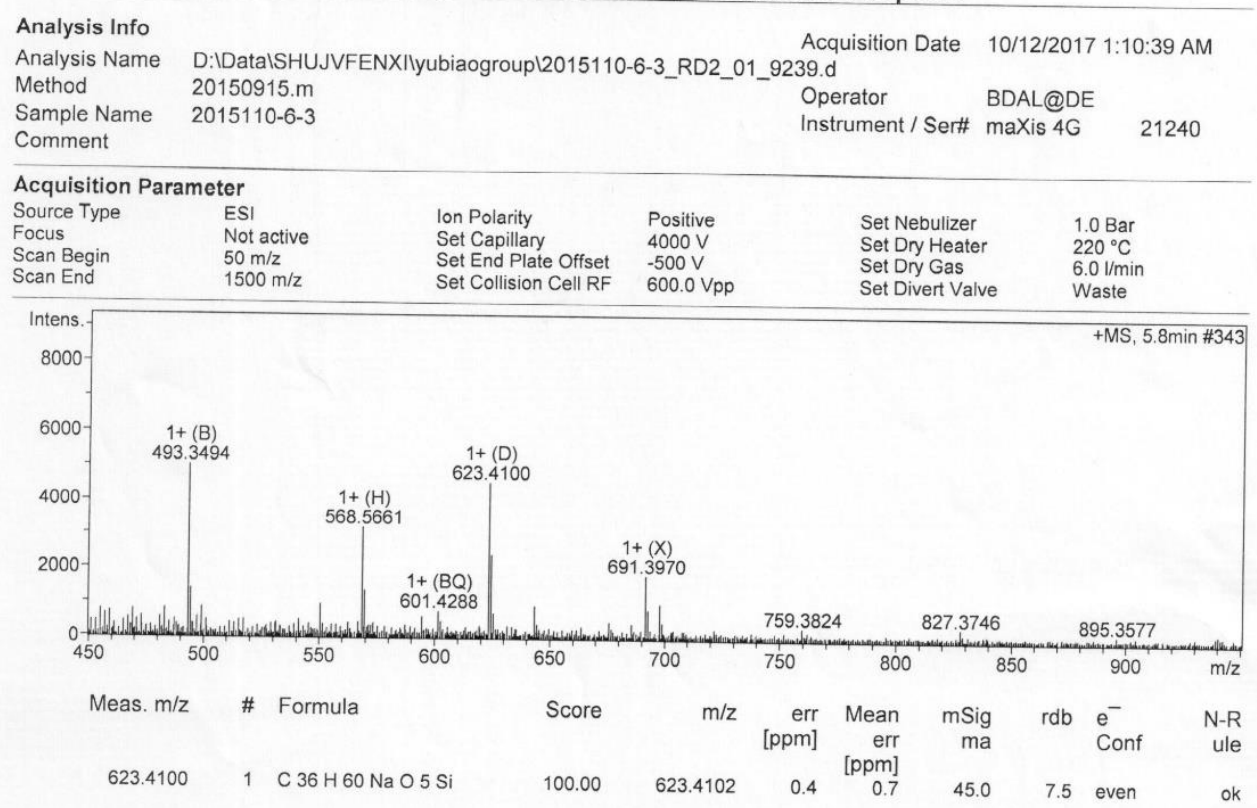

Figure S128. HRMS of compound 16 


\section{Mass Spectrum SmartFormula Report}

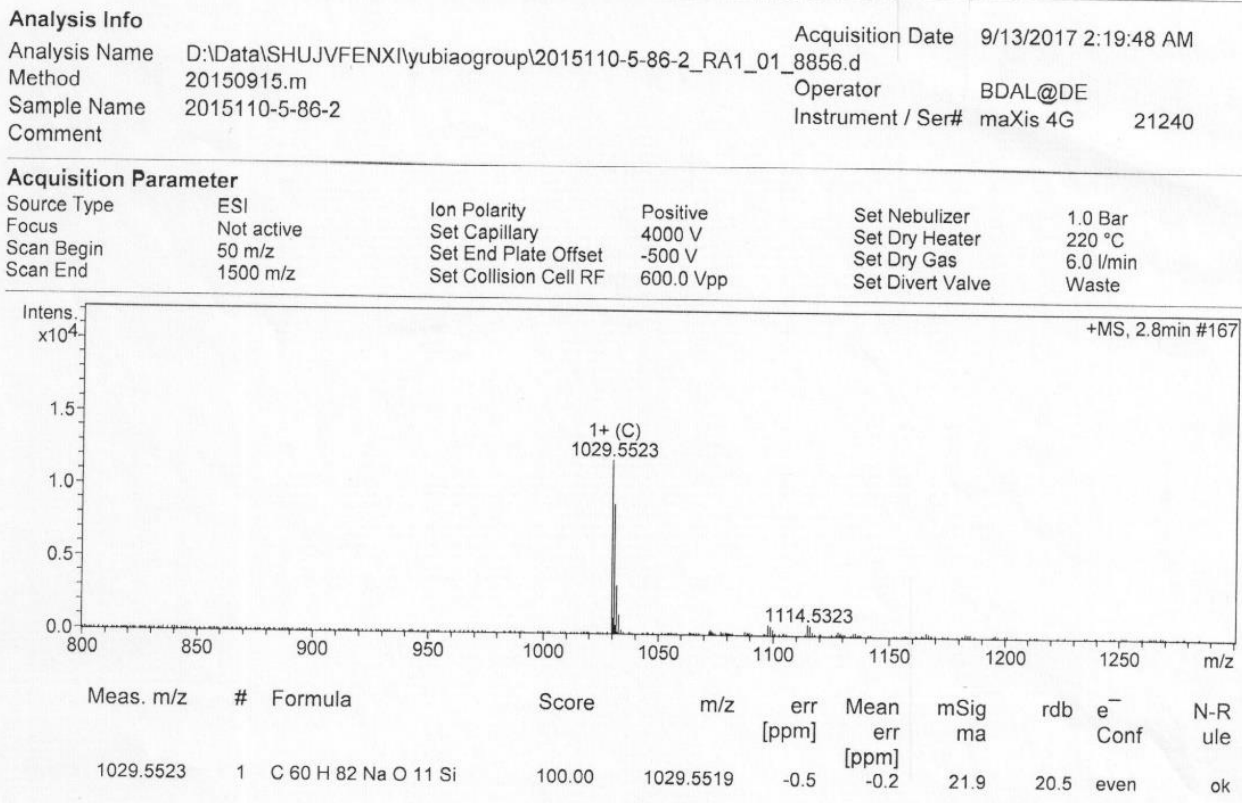

Figure S129. HRMS of compound 18 


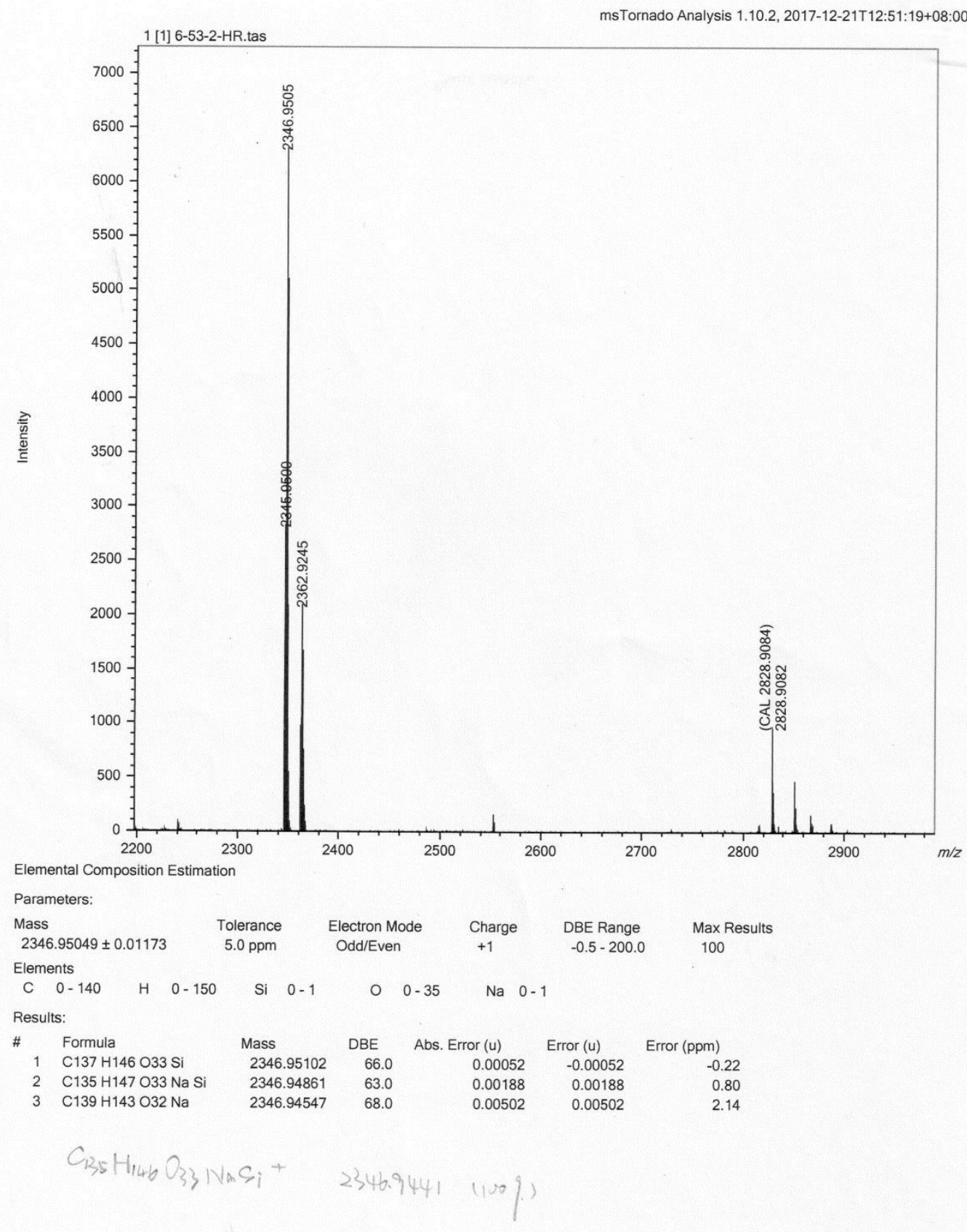

$1 / 1$

Figure S130. HRMS of compound 20 


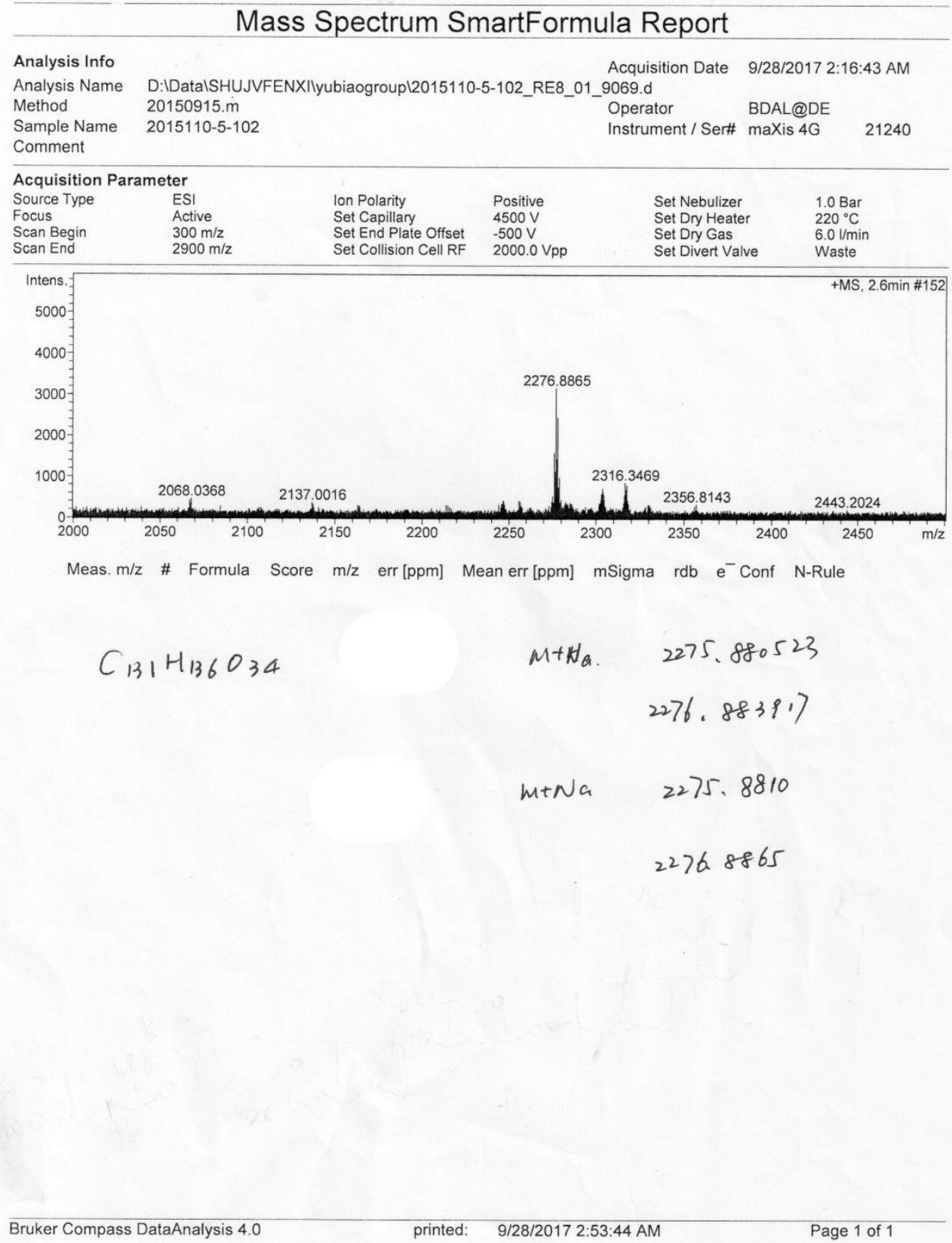

Figure S131. HRMS of compound 21 


\section{Mass Spectrum SmartFormula Report}

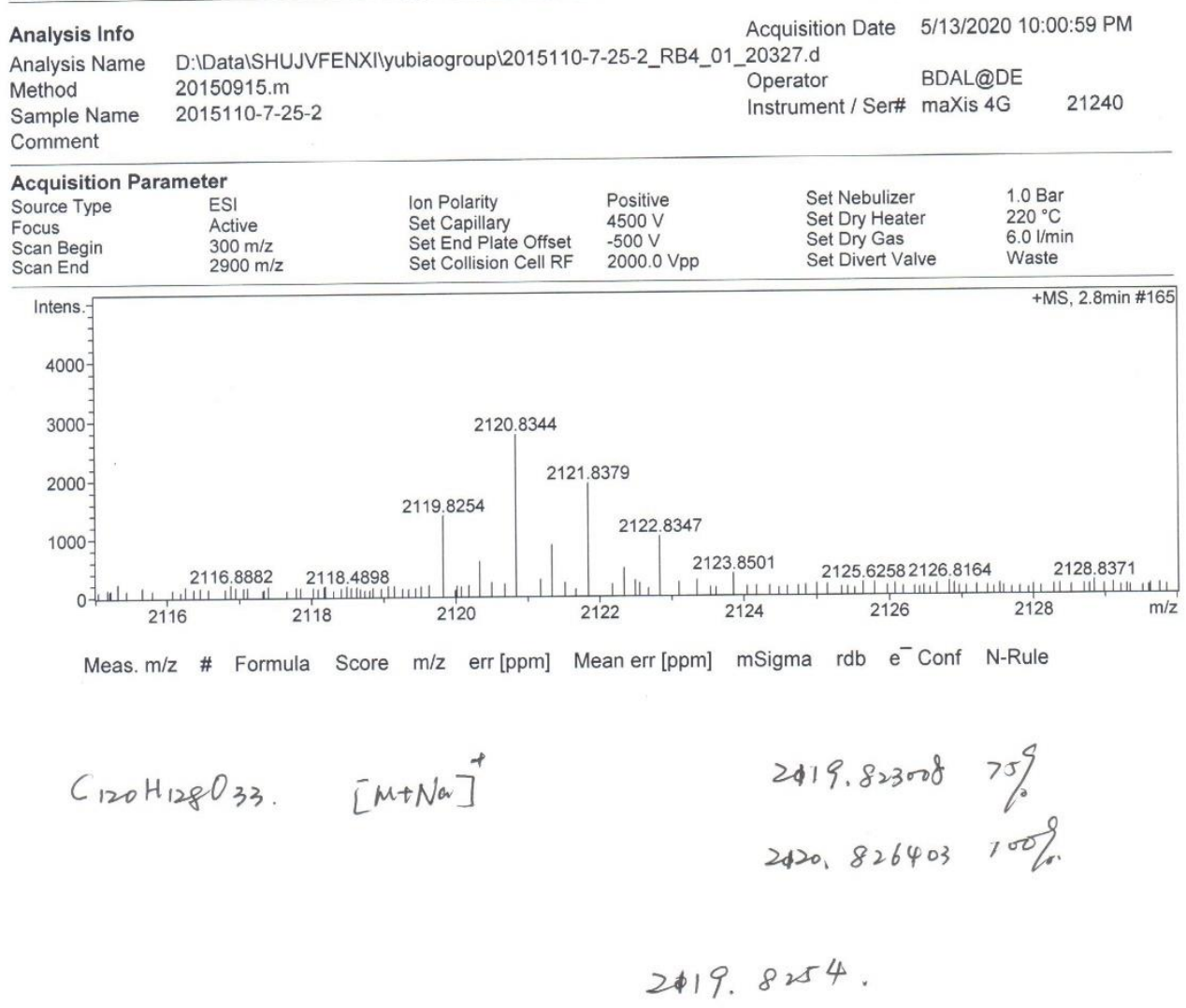

Figure S132. HRMS of compound 22 


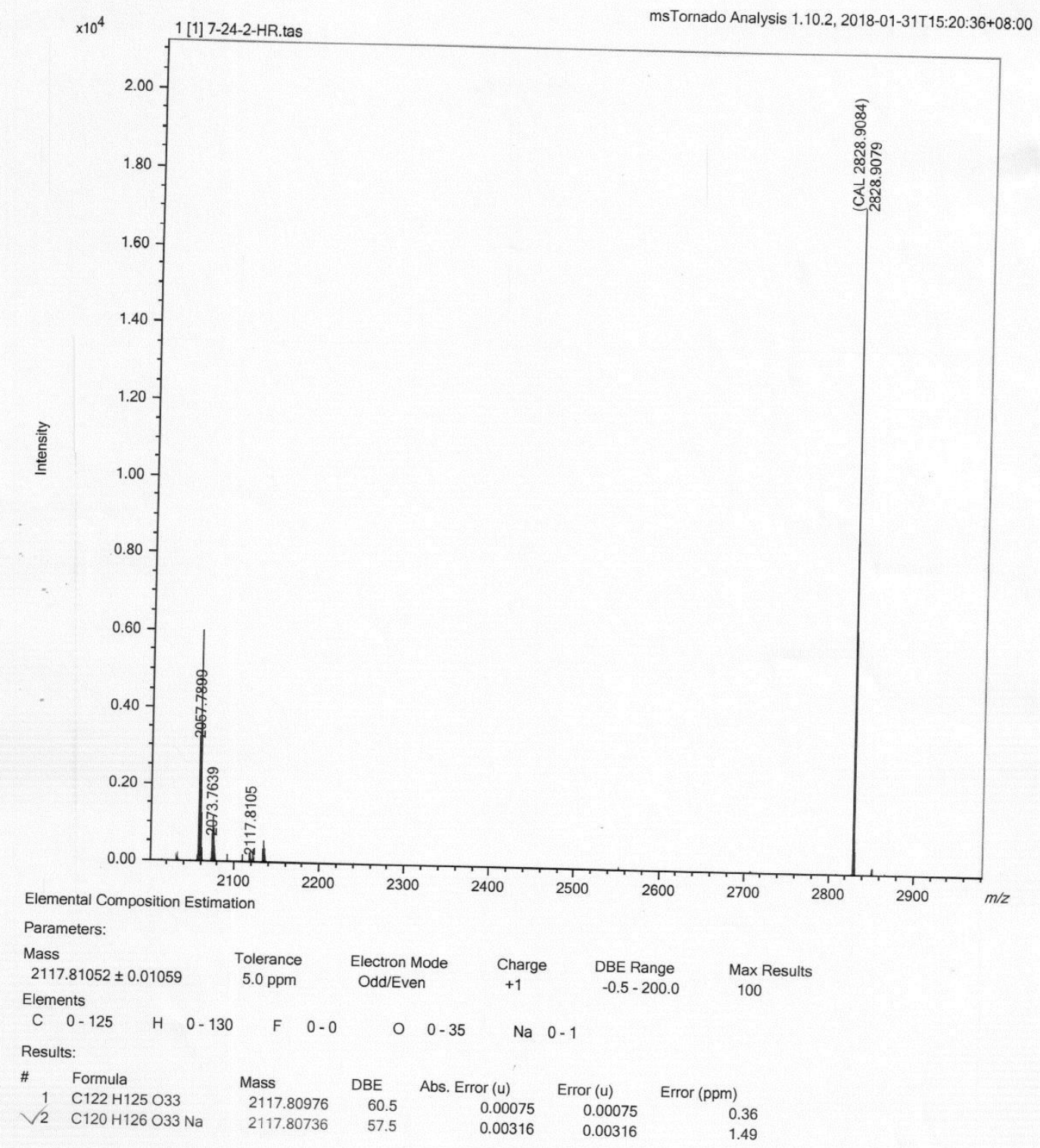

$1 / 1$

Figure S133. HRMS of compound 23 


\section{Mass Spectrum SmartFormula Report}

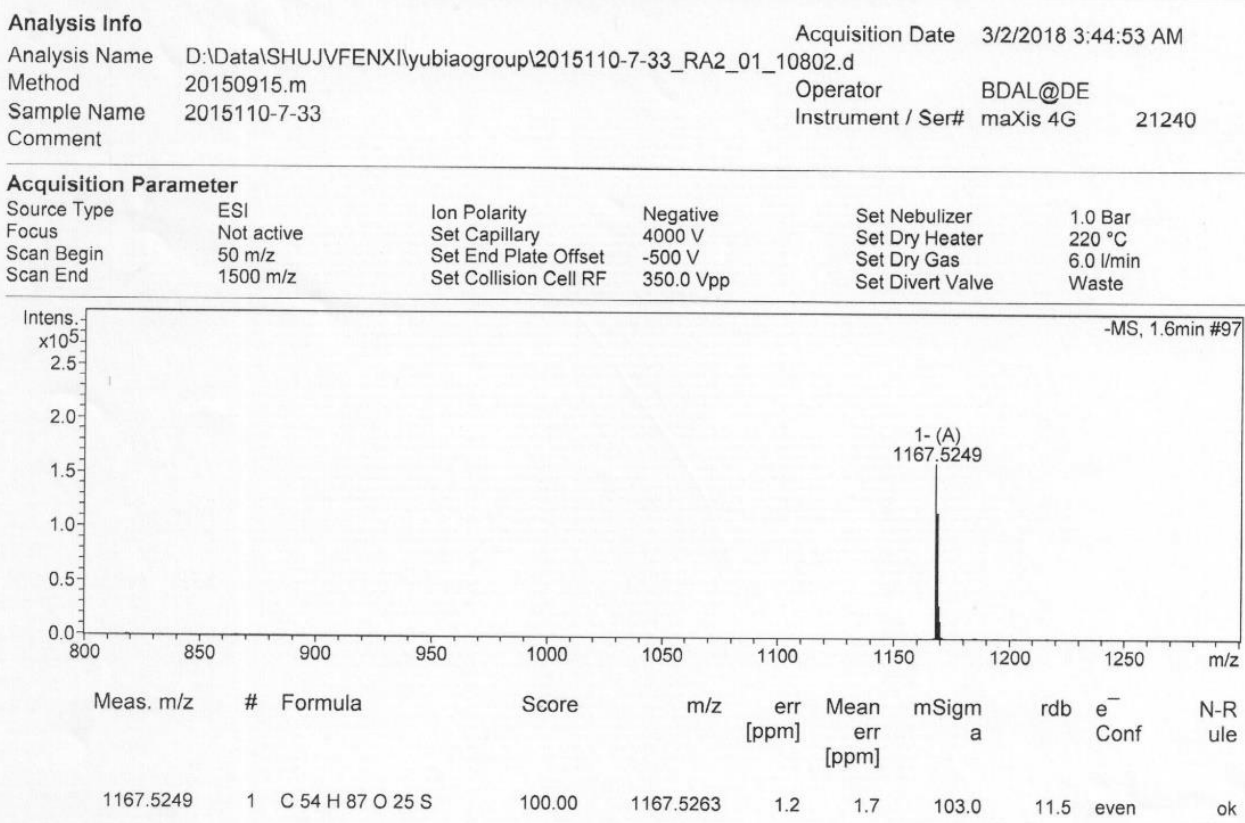

Figure S134. HRMS of compound 3 


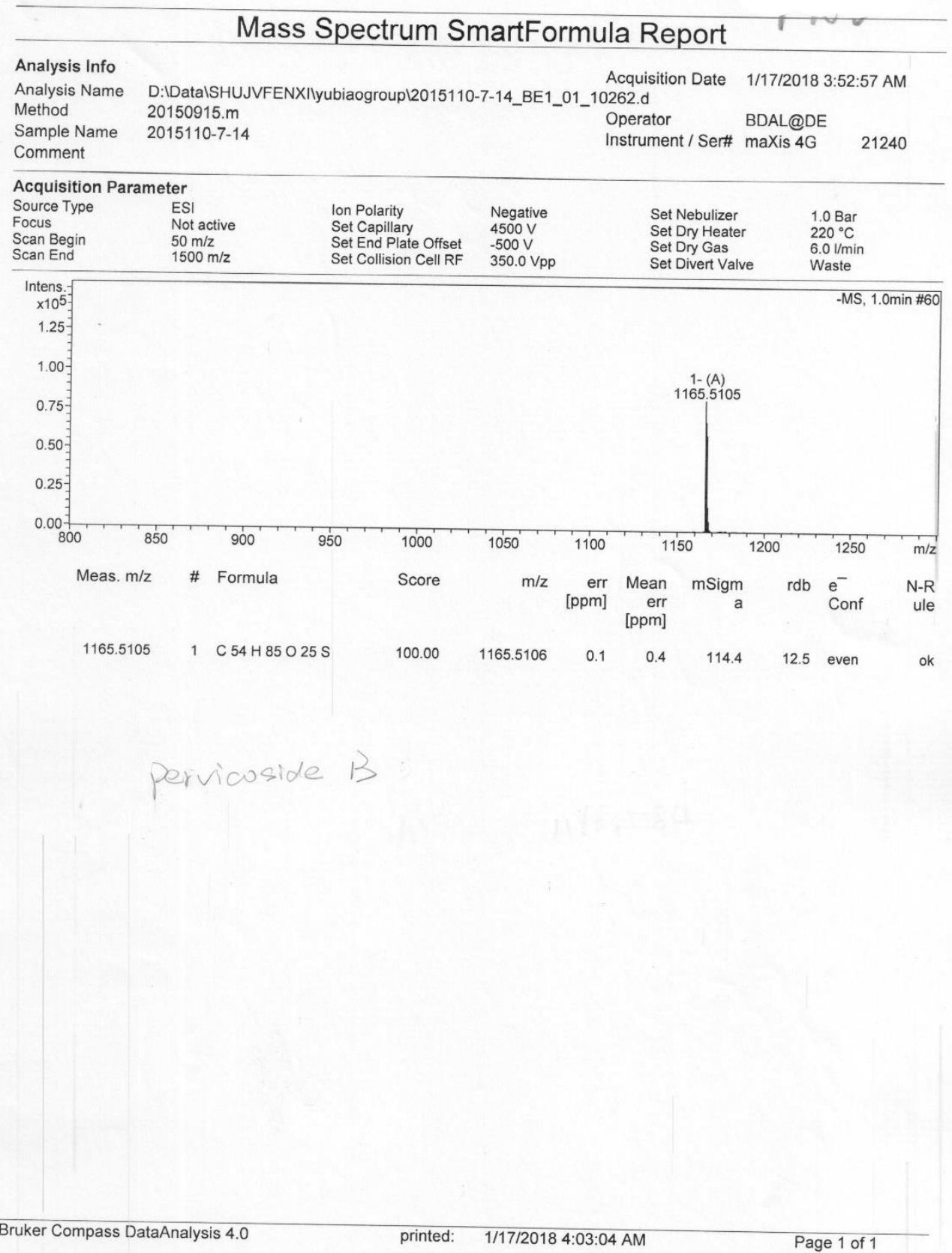

Figure S135. HRMS of compound 2 


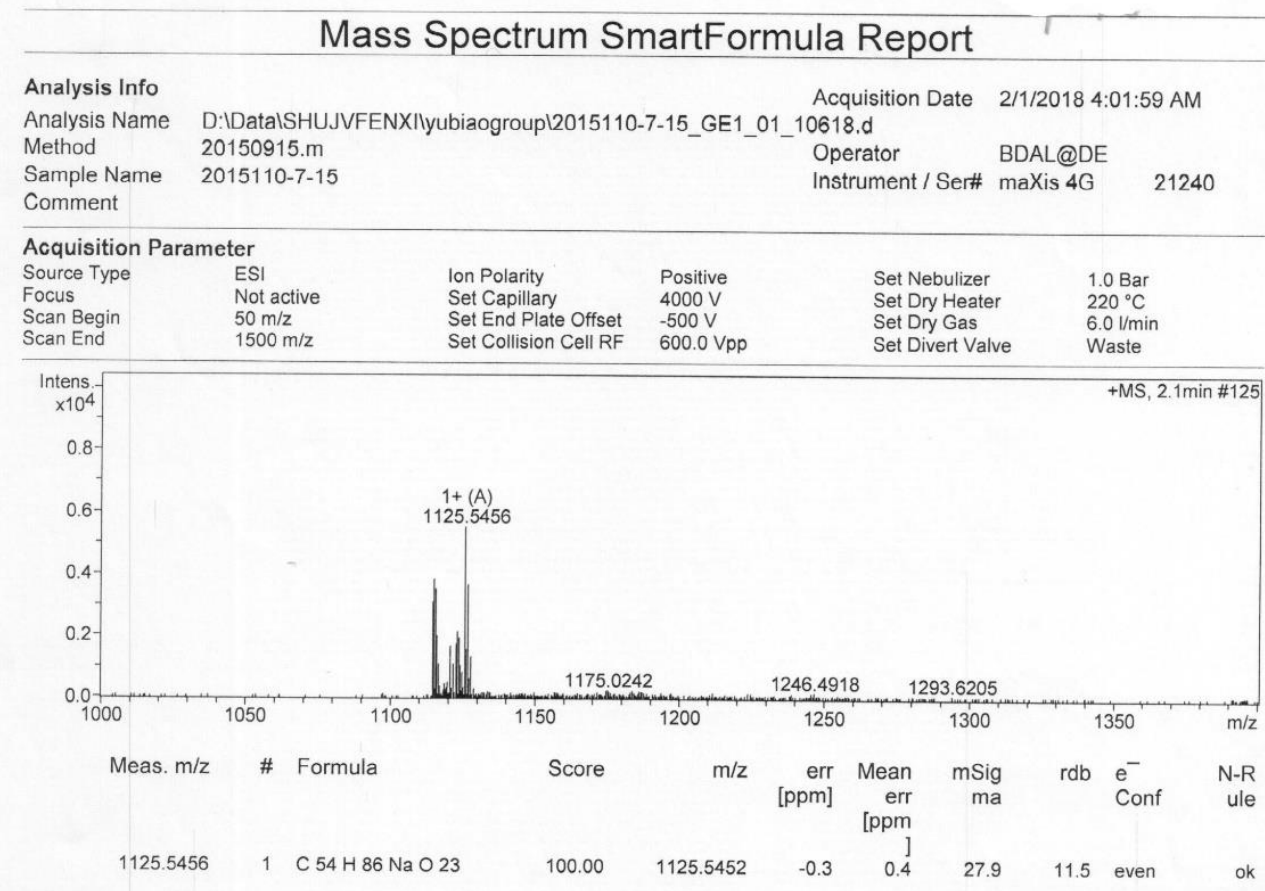

Figure S136. HRMS of compound 24 


\section{Mass Spectrum SmartFormula Report}

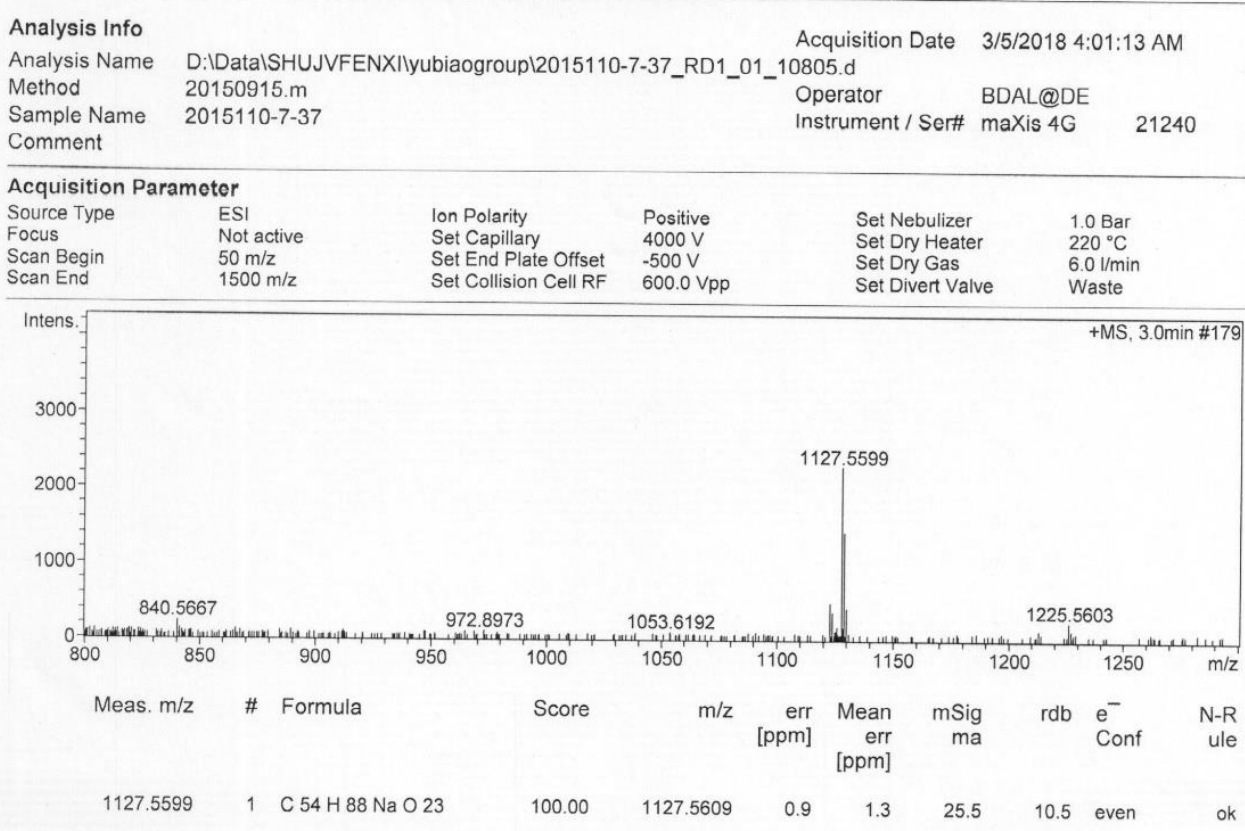

Figure S137. HRMS of compound 25 


\section{Mass Spectrum SmartFormula Report}

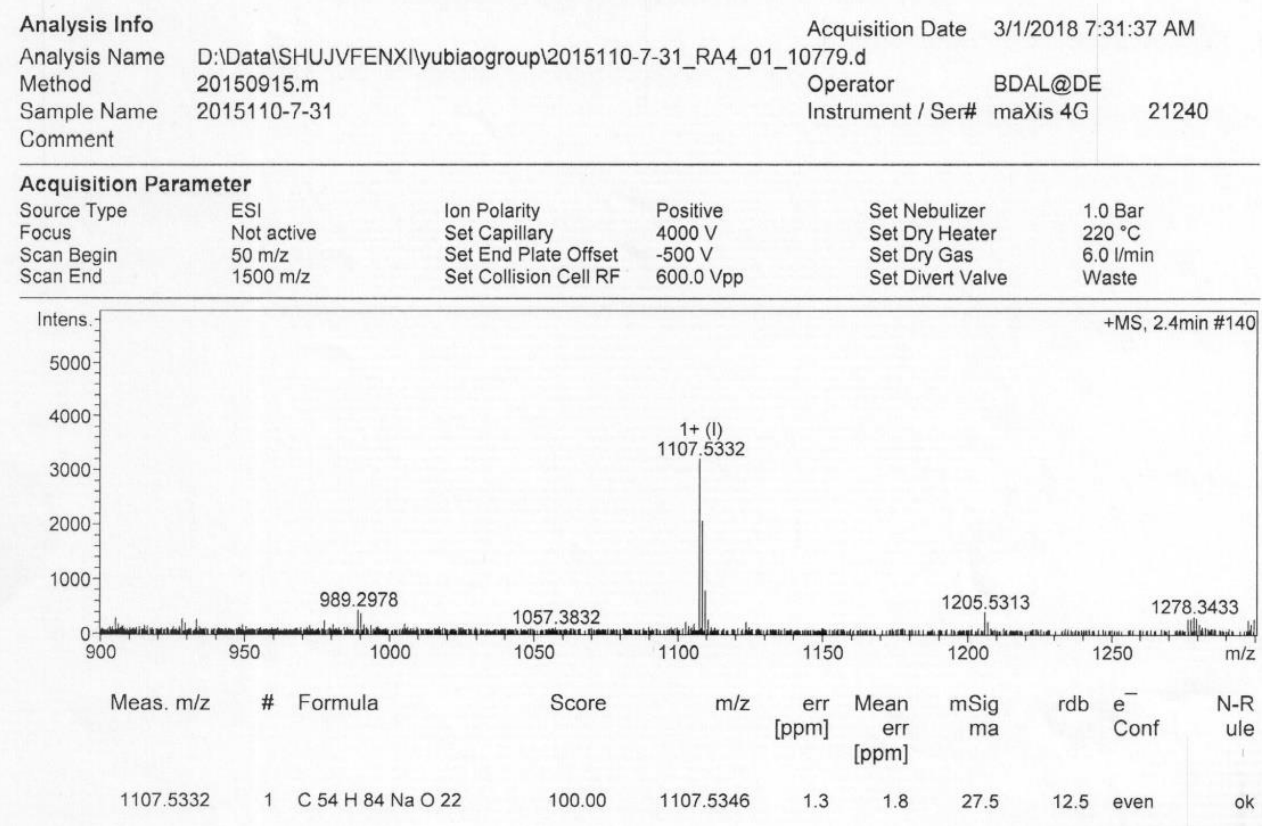

Figure S138. HRMS of compound 26 


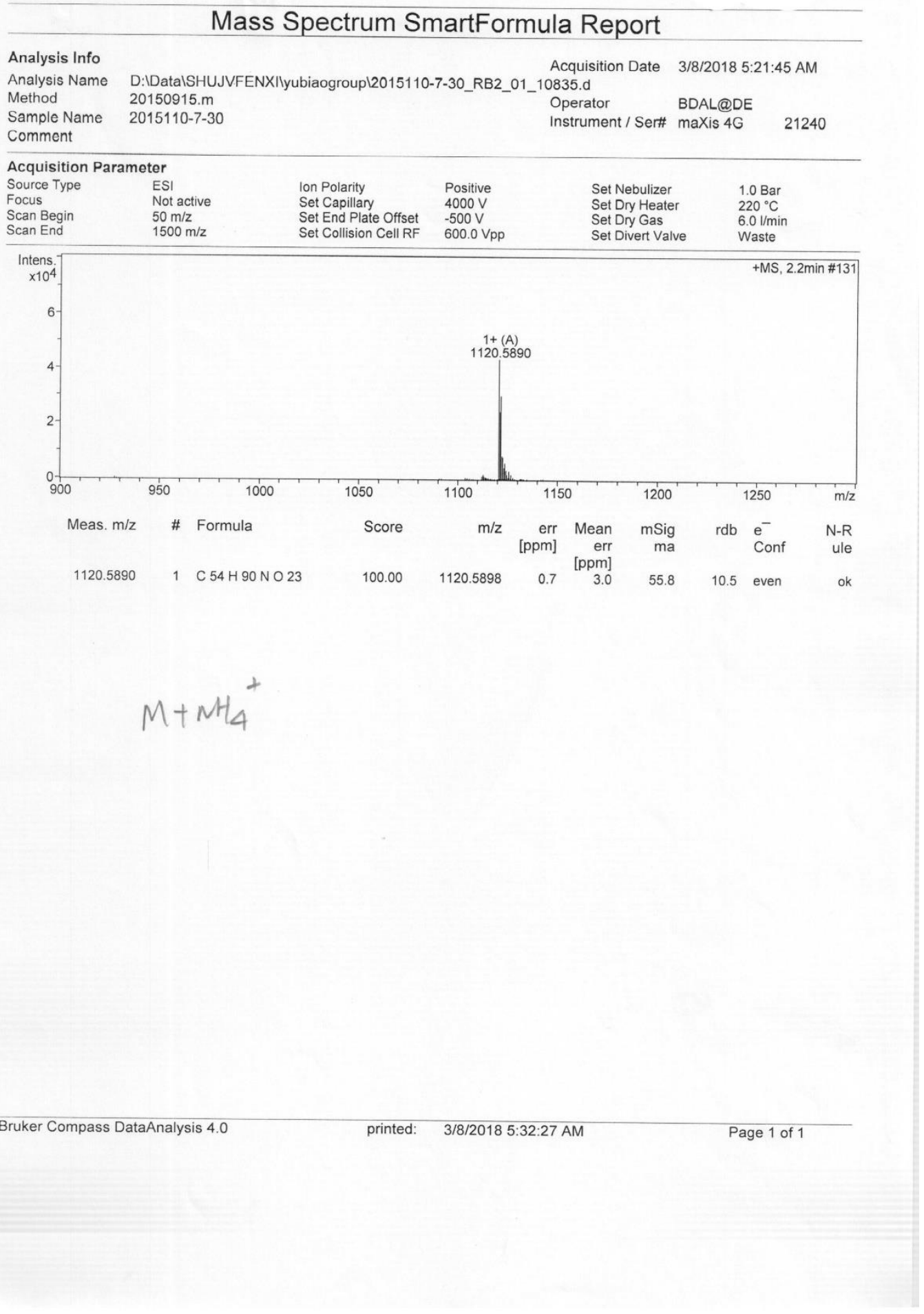

Figure S139. HRMS of compound 27 


\section{Mass Spectrum SmartFormula Report}

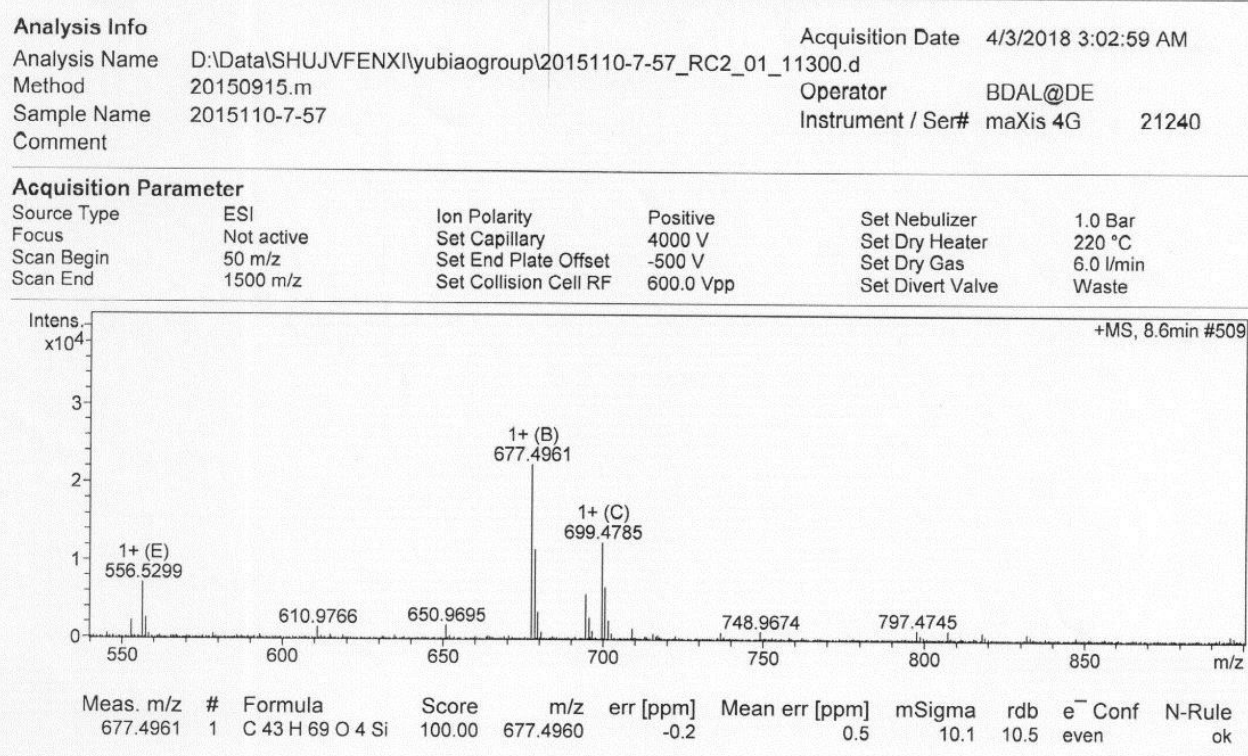

Figure S140. HRMS of compound 28 


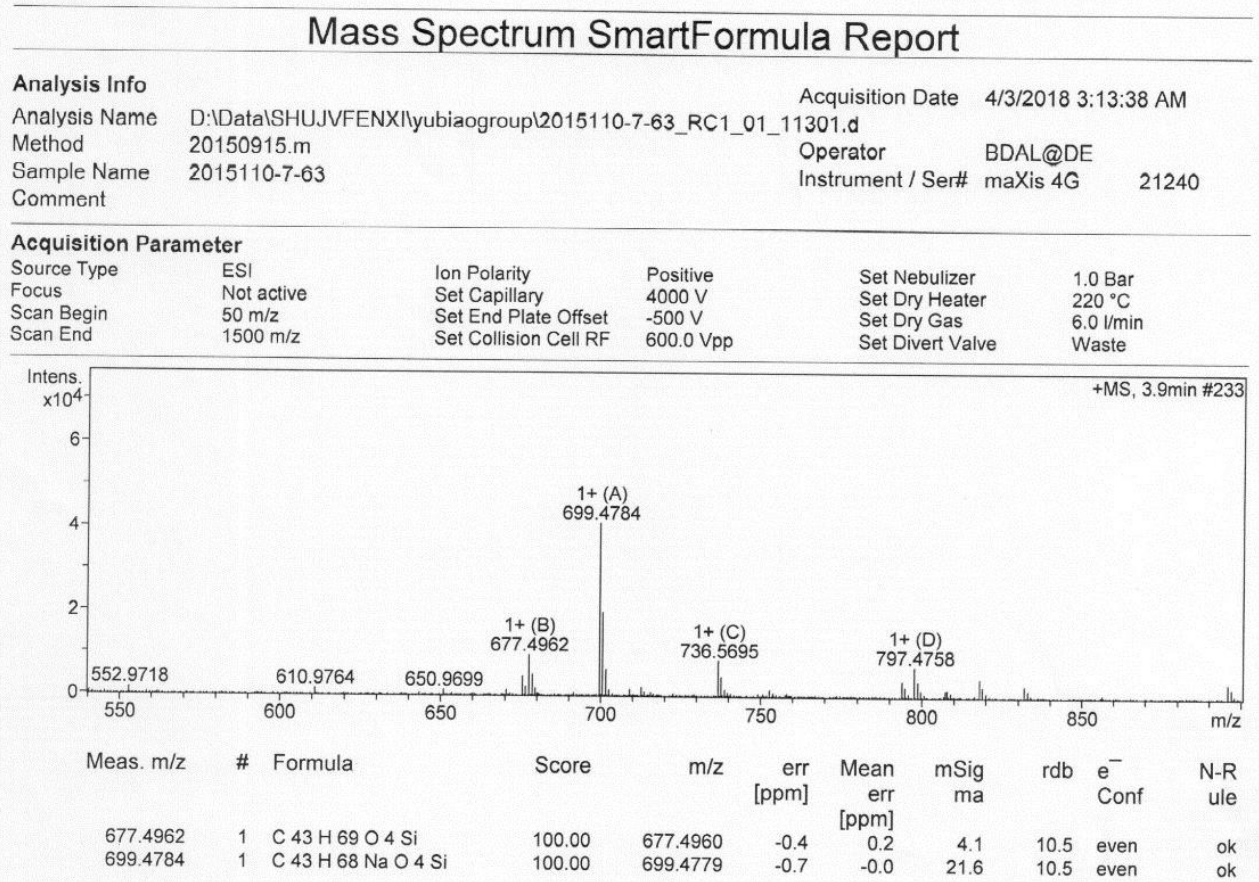

Figure S141. HRMS of compound 29 


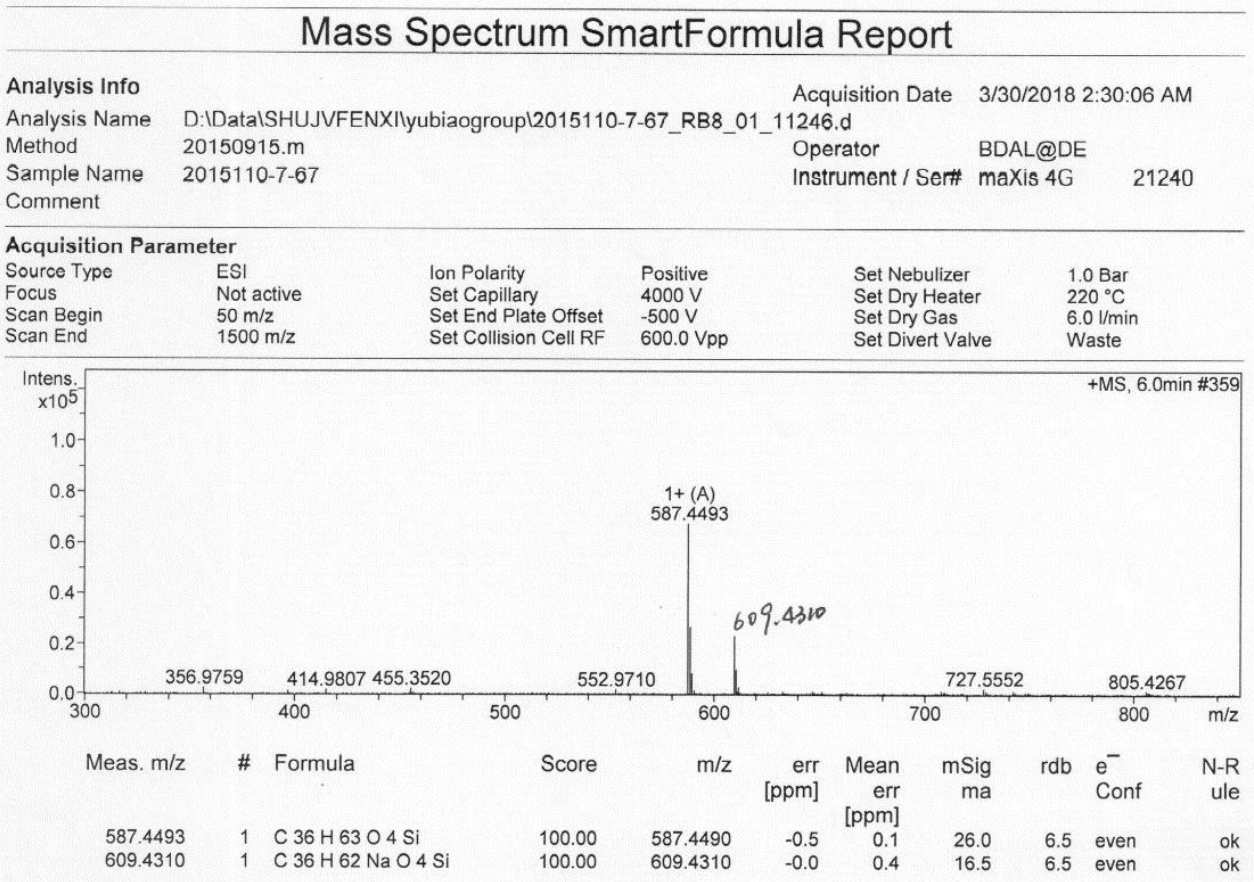

Figure S142. HRMS of compound 30 


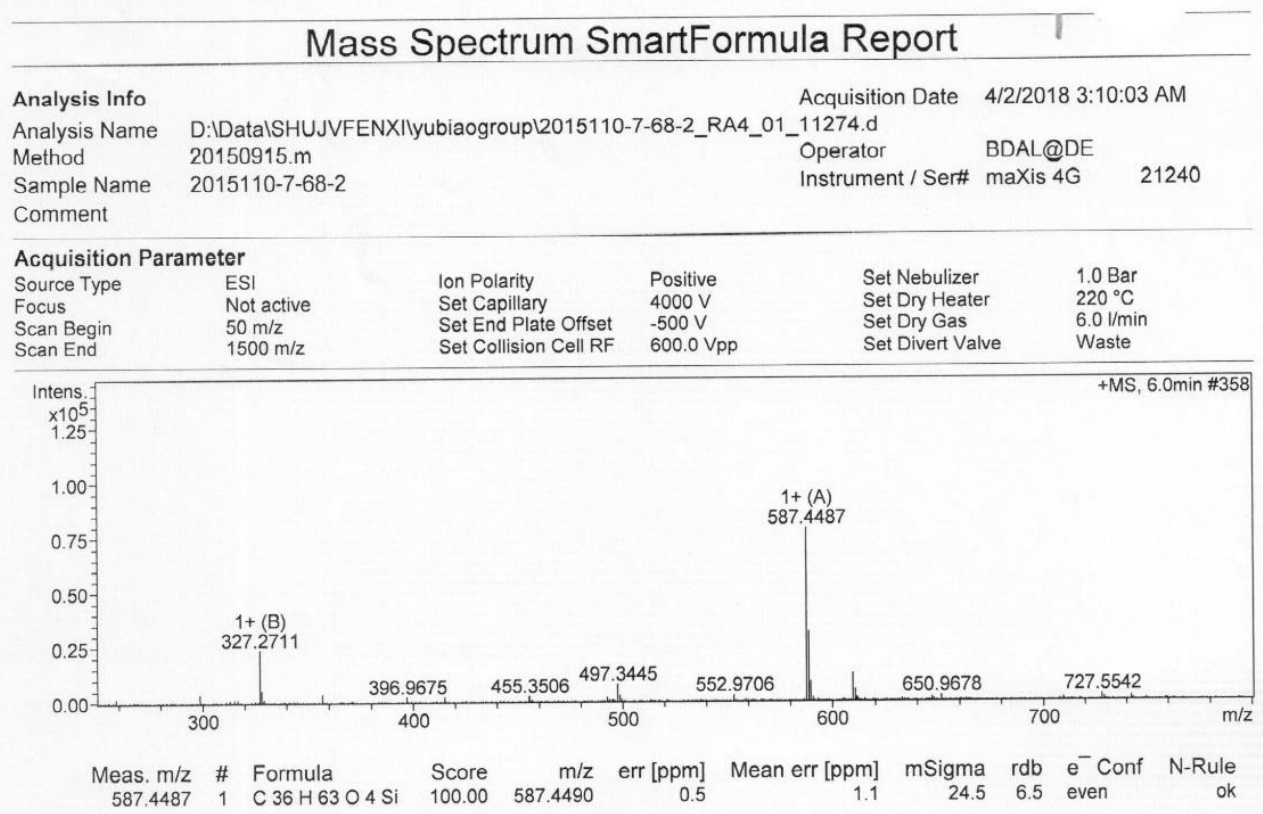

Figure S143. HRMS of compound 31 


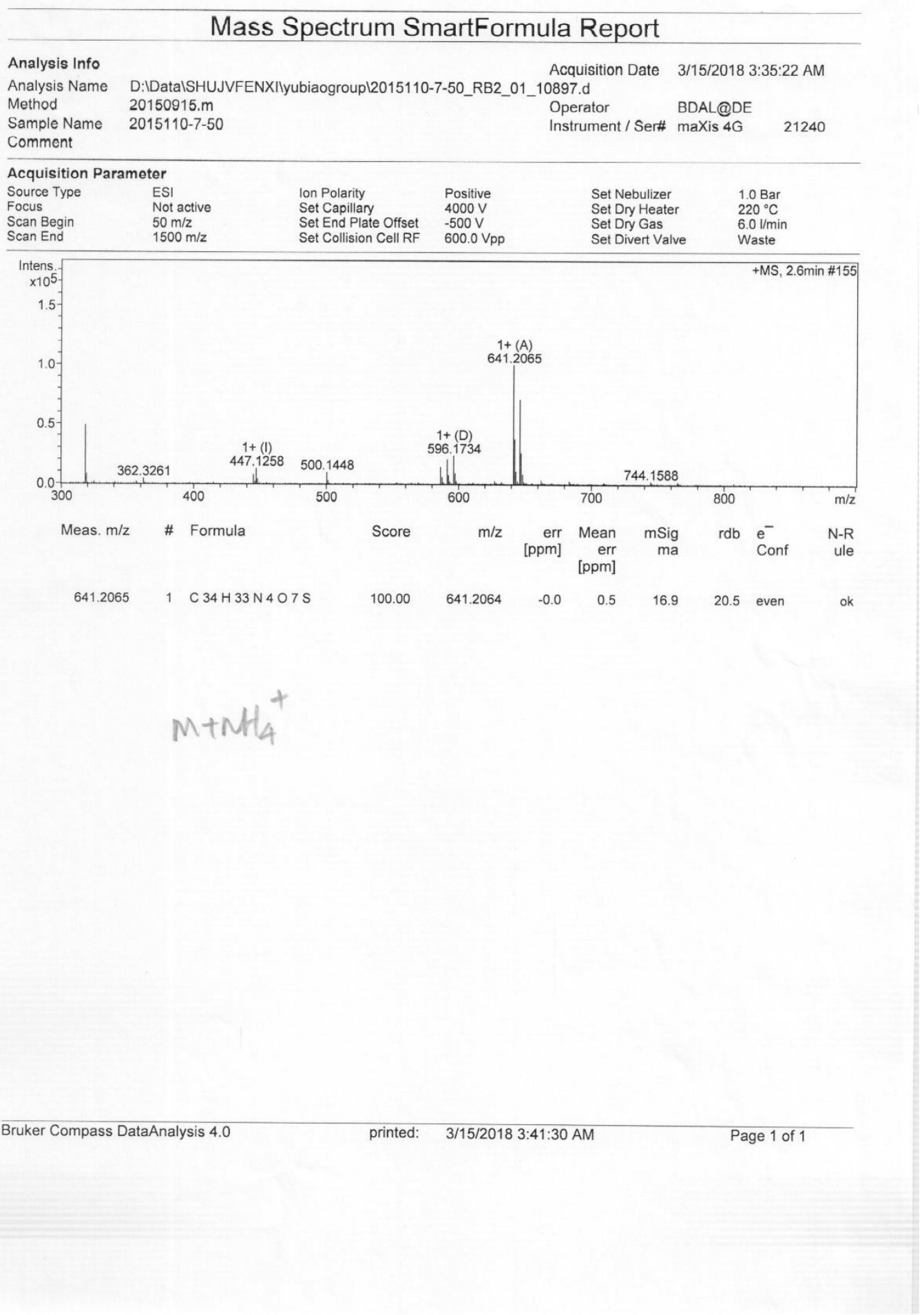

Figure S144. HRMS of $p$-tolyl 2- $O$-Azmb-3,4-di- $O$-benzoyl-1-thio- $\beta$-D-

xylopyranoside 


\section{Mass Spectrum SmartFormula Report}

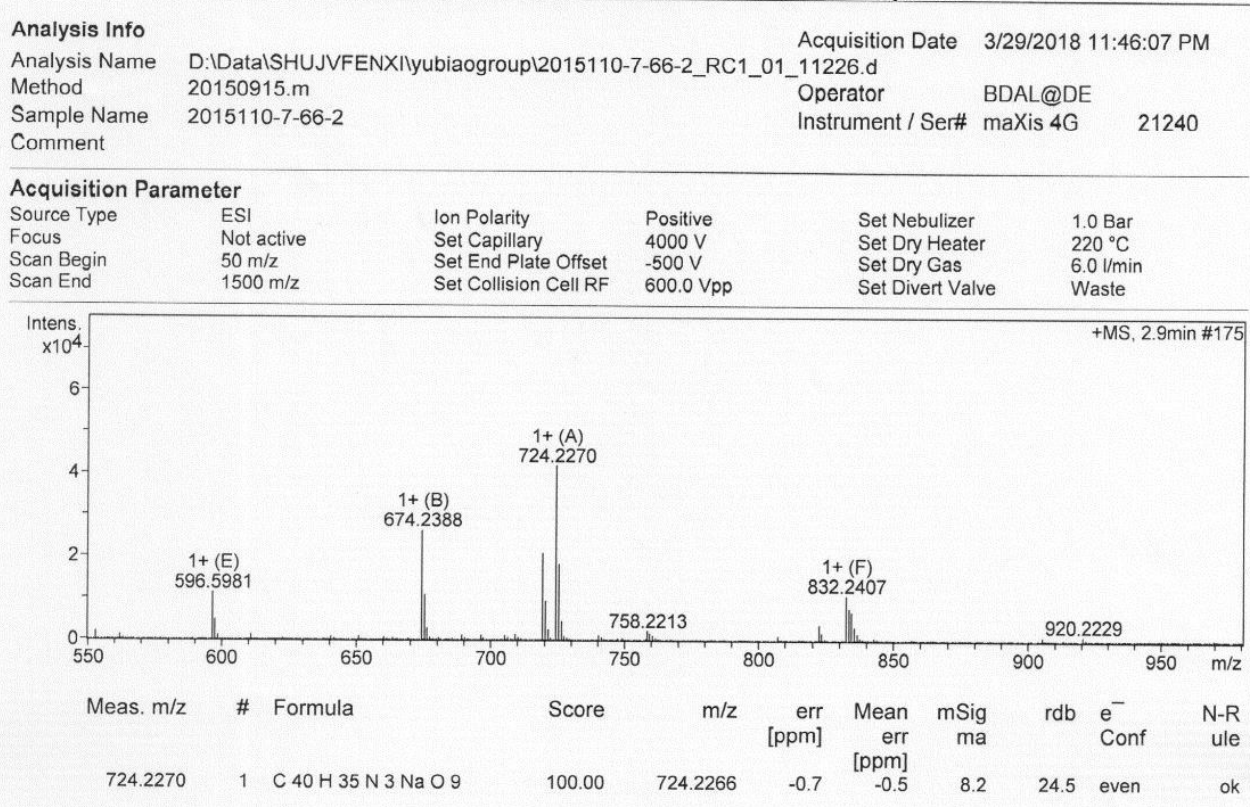

Figure S145. HRMS of compound 32a 


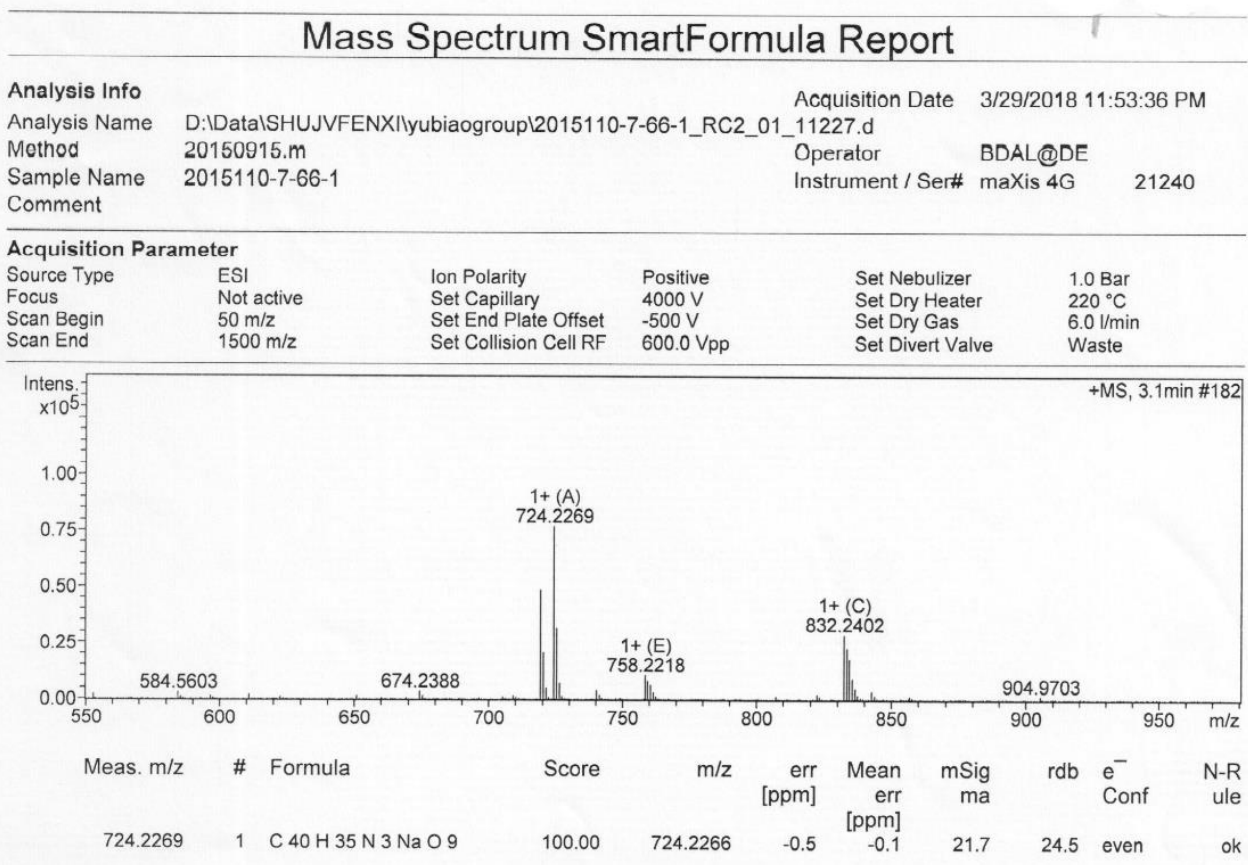

Figure S146. HRMS of compound $32 \beta$ 


\section{Mass Spectrum SmartFormula Report}

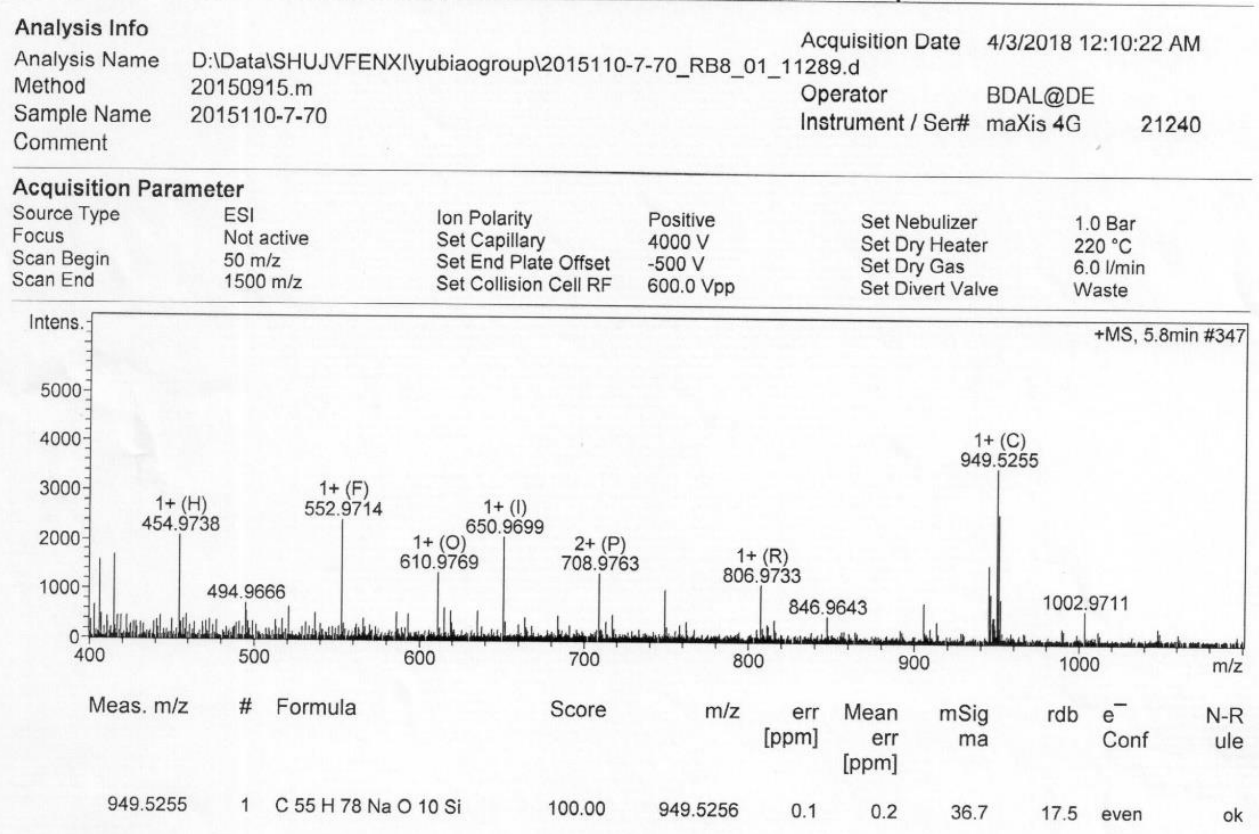

Figure S147. HRMS of compound 33 


\section{Mass Spectrum SmartFormula Report}

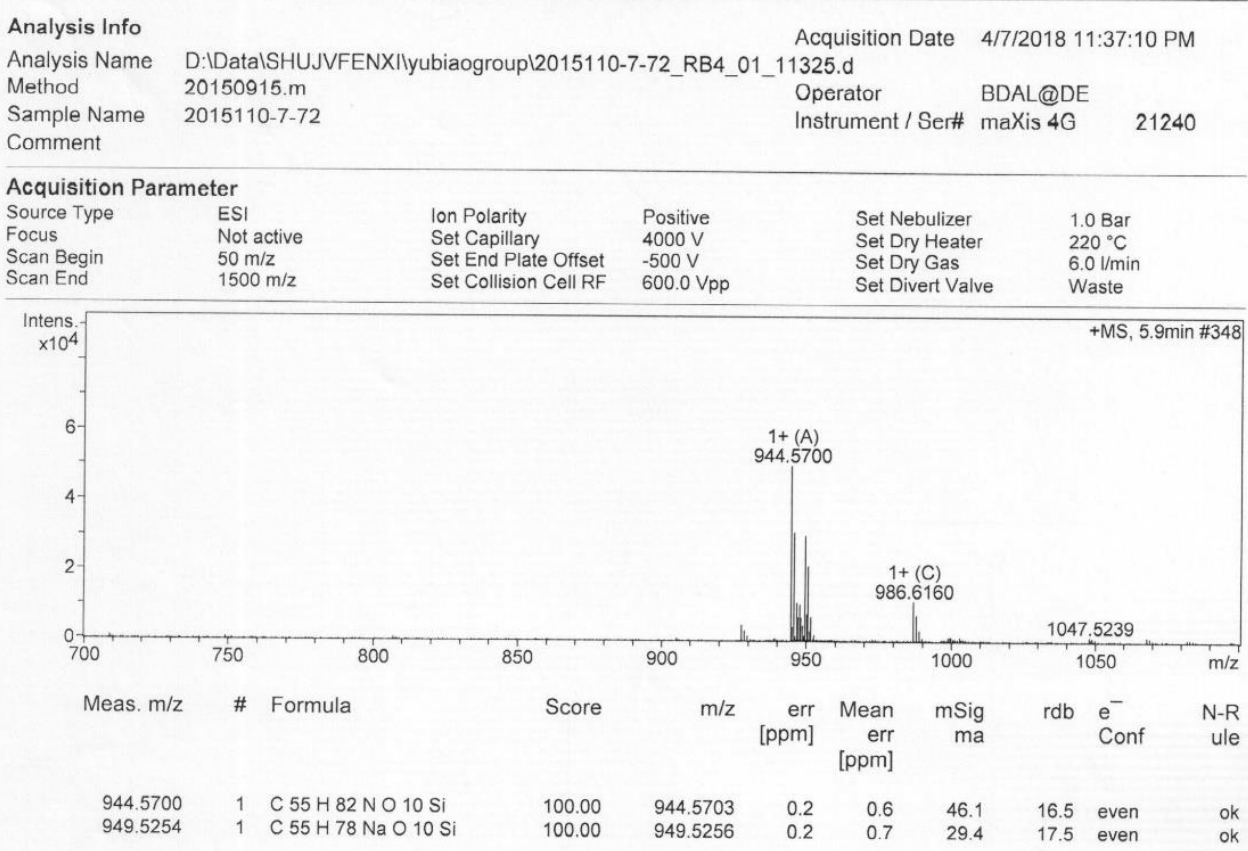

Figure S148. HRMS of compound 34 


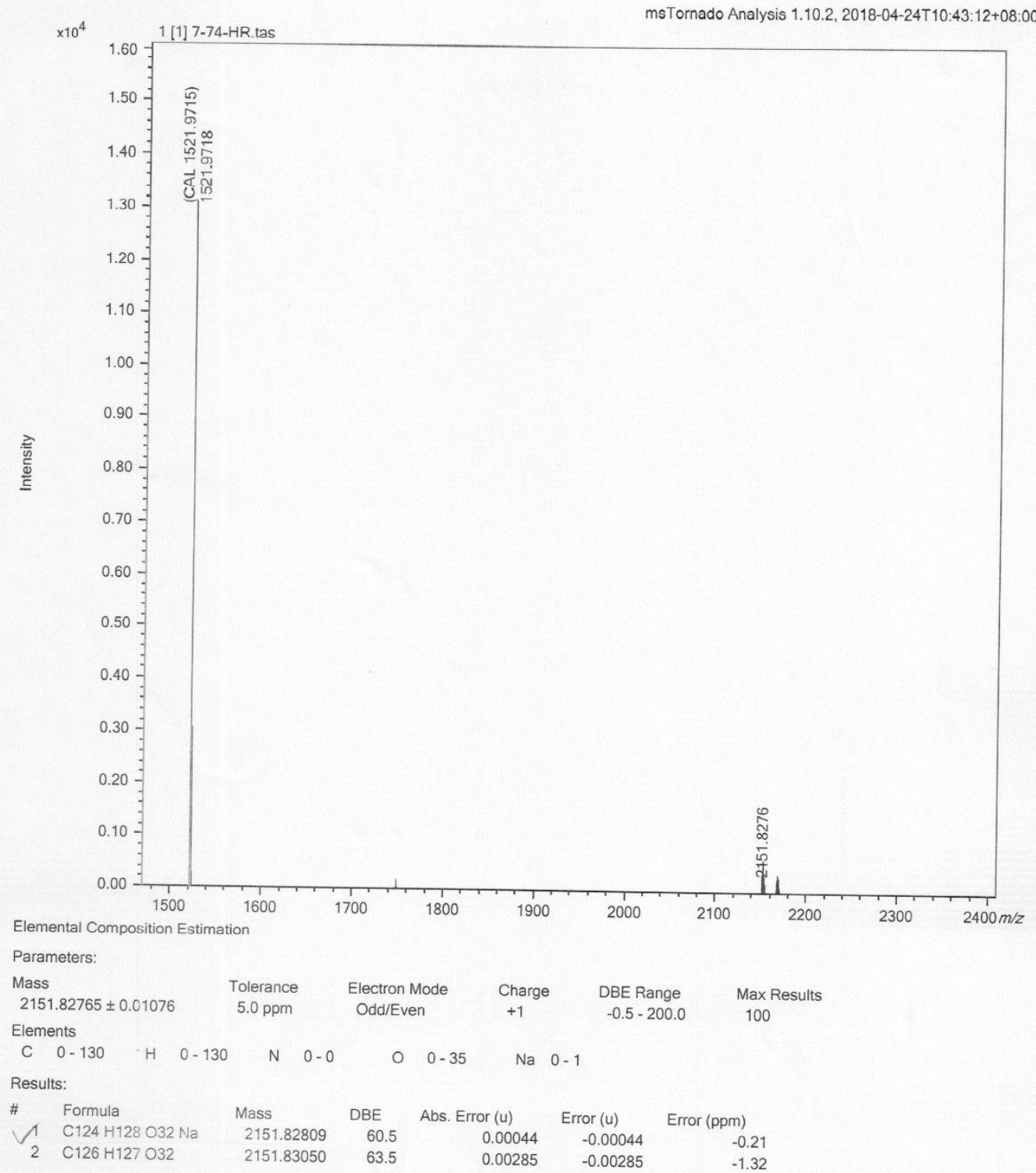

Figure S149. HRMS of compound 35 


\section{Mass Spectrum SmartFormula Report}

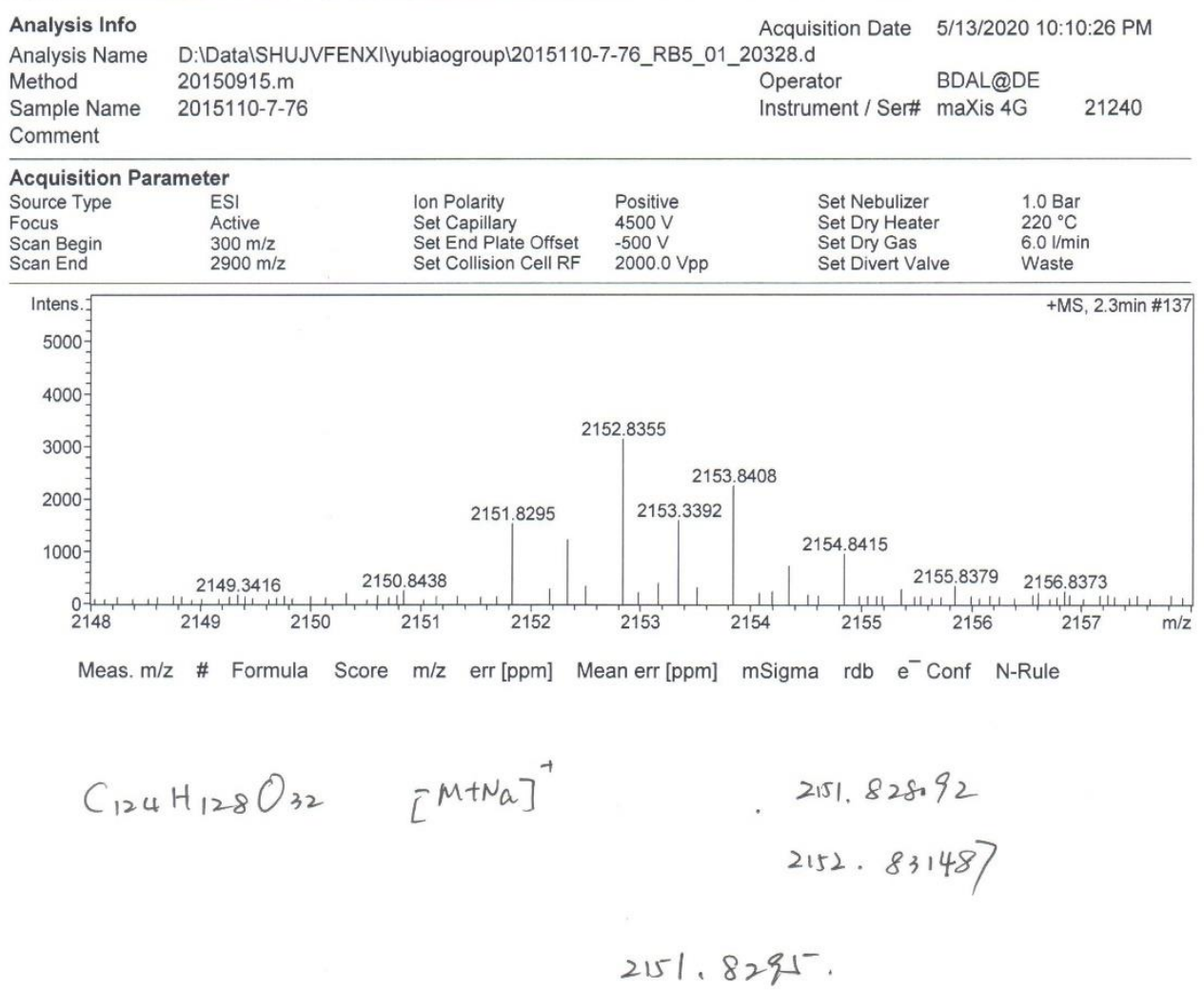

Figure S150. HRMS of compound 36 


\section{Mass Spectrum SmartFormula Report}

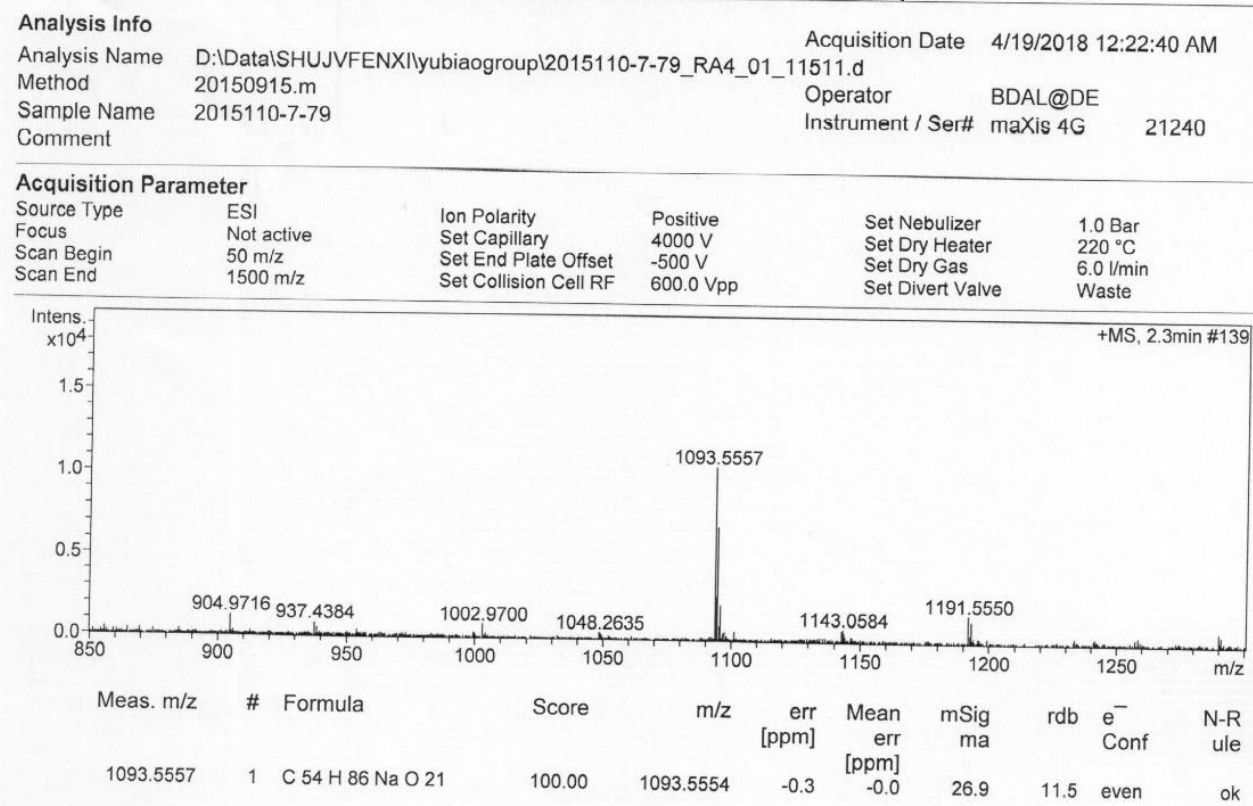

Figure S151. HRMS of compound 37 


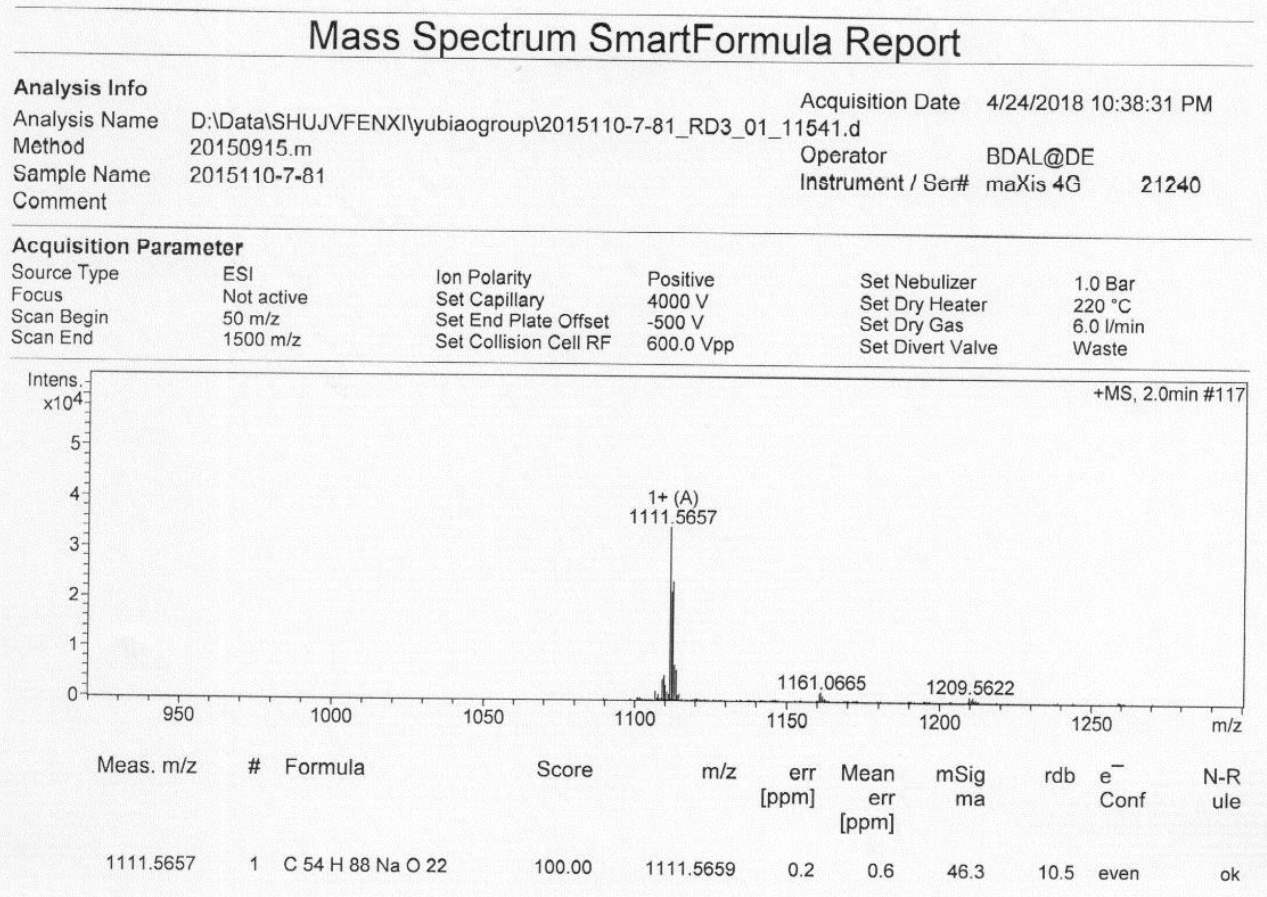

Figure S152. HRMS of compound 38 


\section{Mass Spectrum SmartFormula Report}

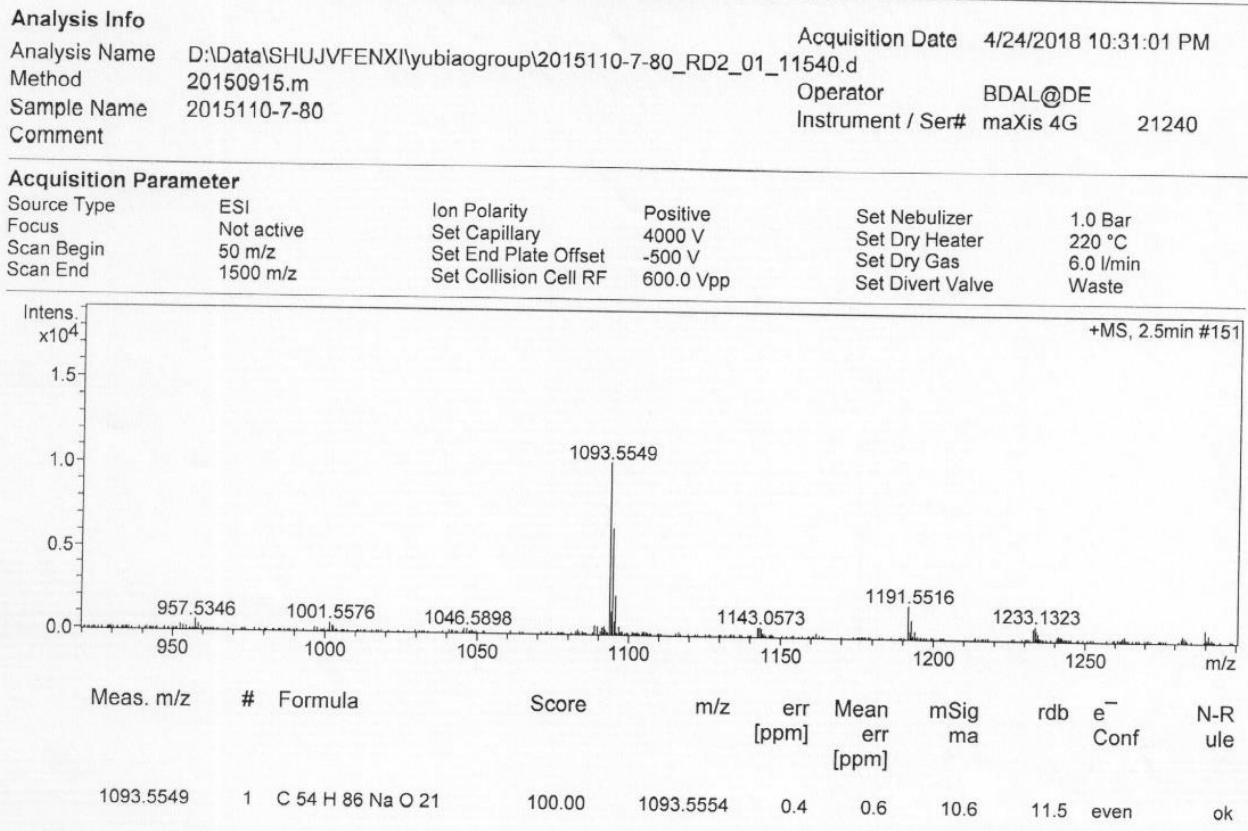

Figure S153. HRMS of compound 39 


\section{Mass Spectrum SmartFormula Report}

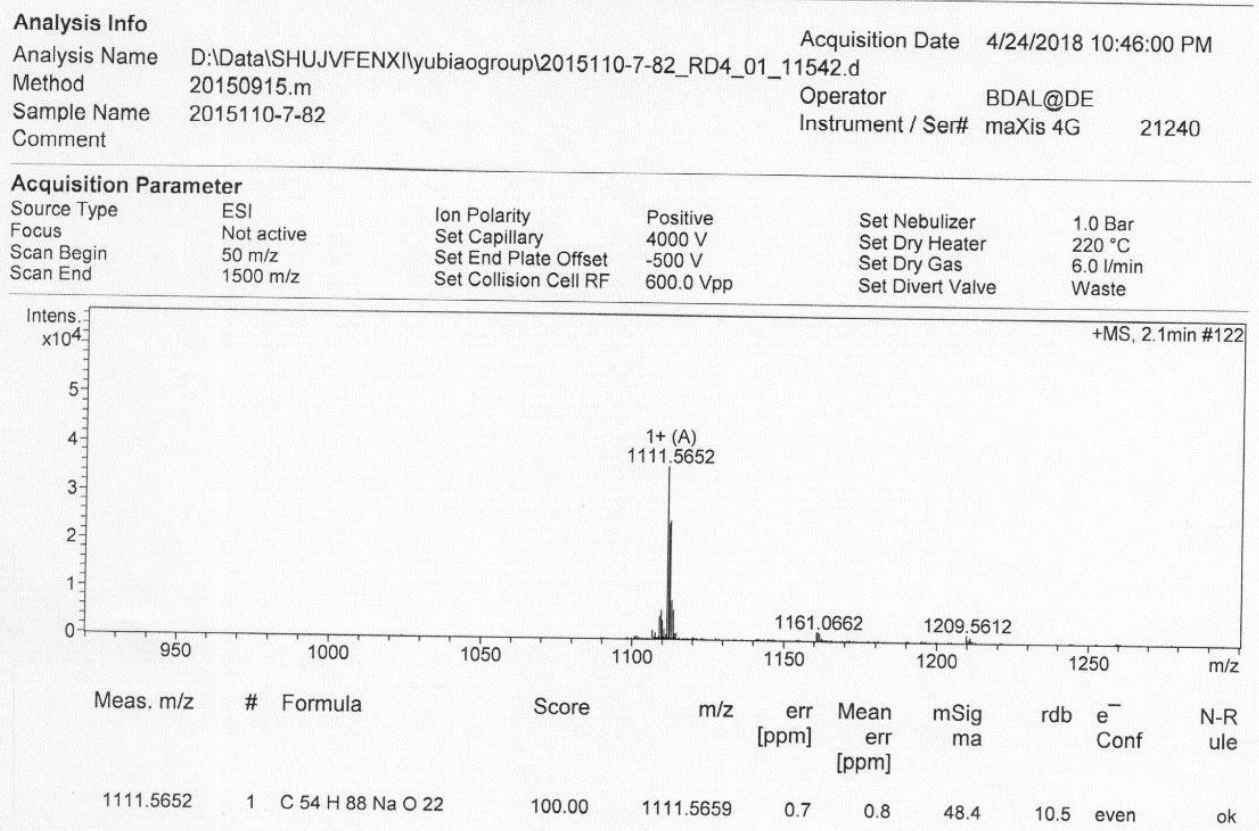

Figure S154. HRMS of compound 40 


\section{Mass Spectrum SmartFormula Report}

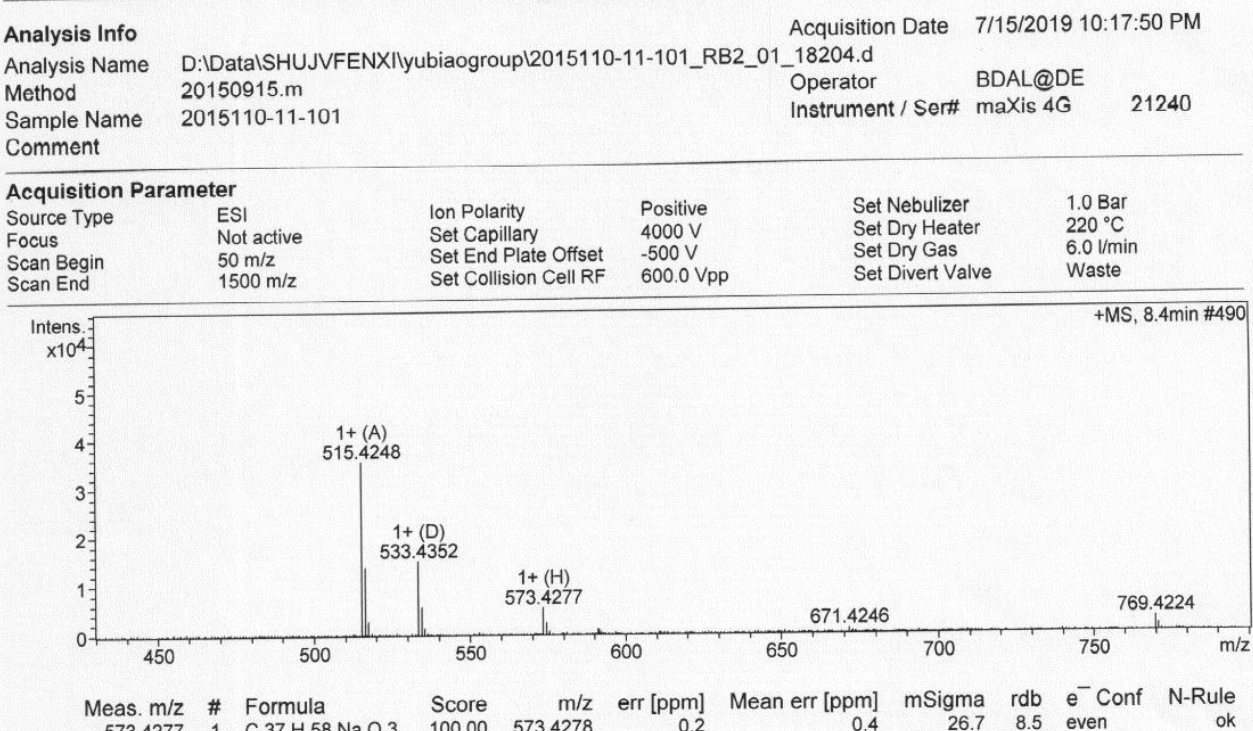

$\begin{array}{llll}573.4277 \quad 1 \quad \mathrm{C} 37 \mathrm{H} 58 \mathrm{NaO} 3 & 100.00 & 573.4278\end{array}$

Figure S155. HRMS of compound 41 


\section{Mass Spectrum SmartFormula Report}

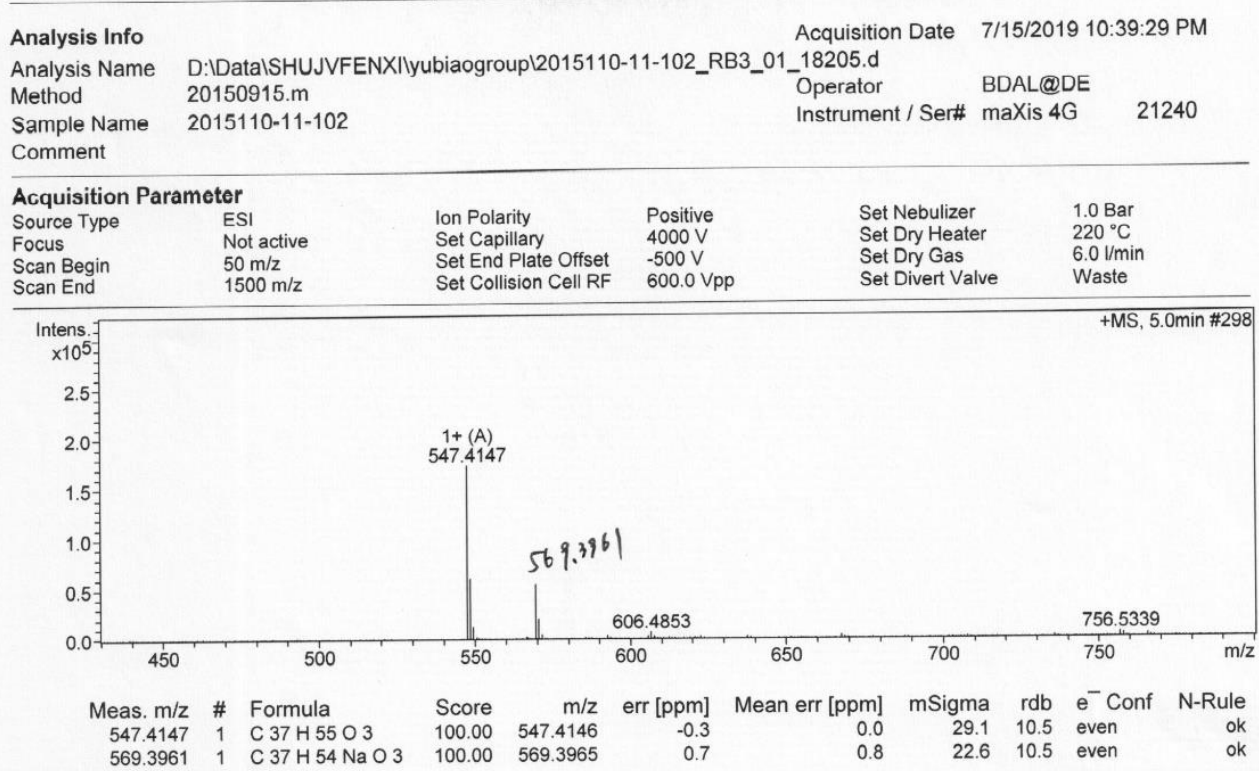

Figure S156. HRMS of compound 42 


\section{Mass Spectrum SmartFormula Report}

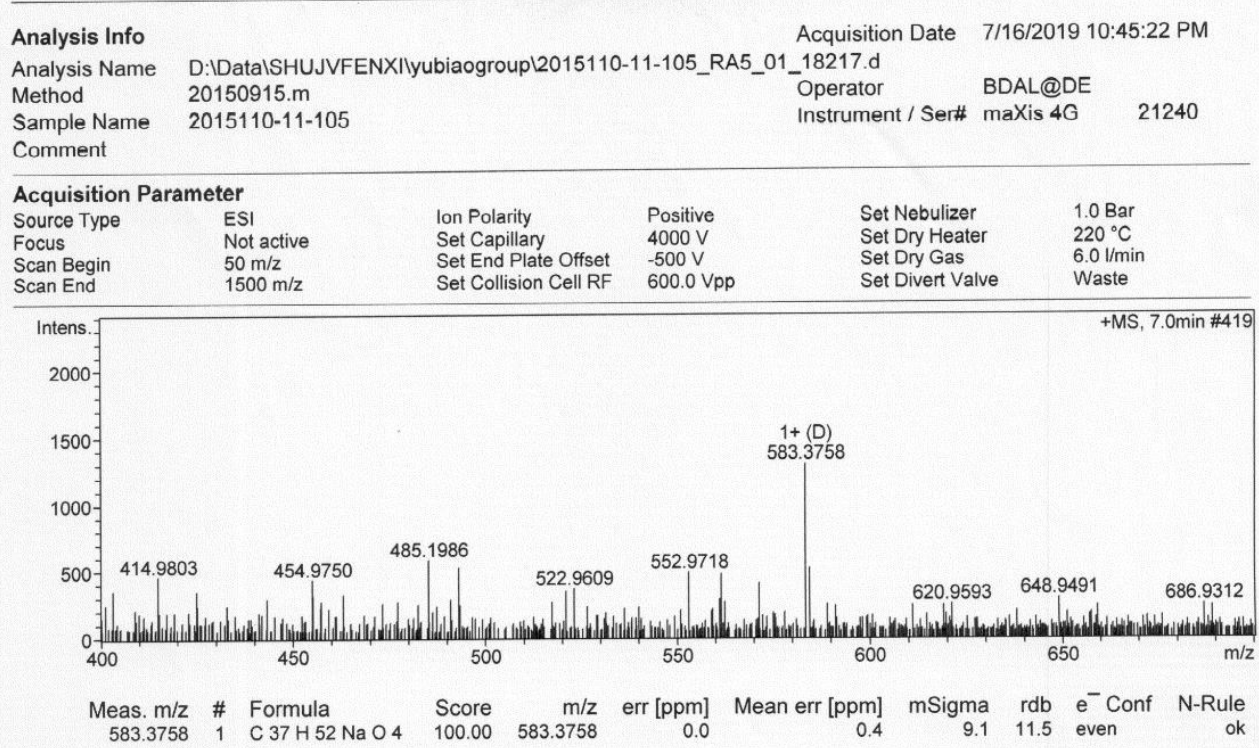

Figure S157. HRMS of compound 43 


\section{Mass Spectrum SmartFormula Report}

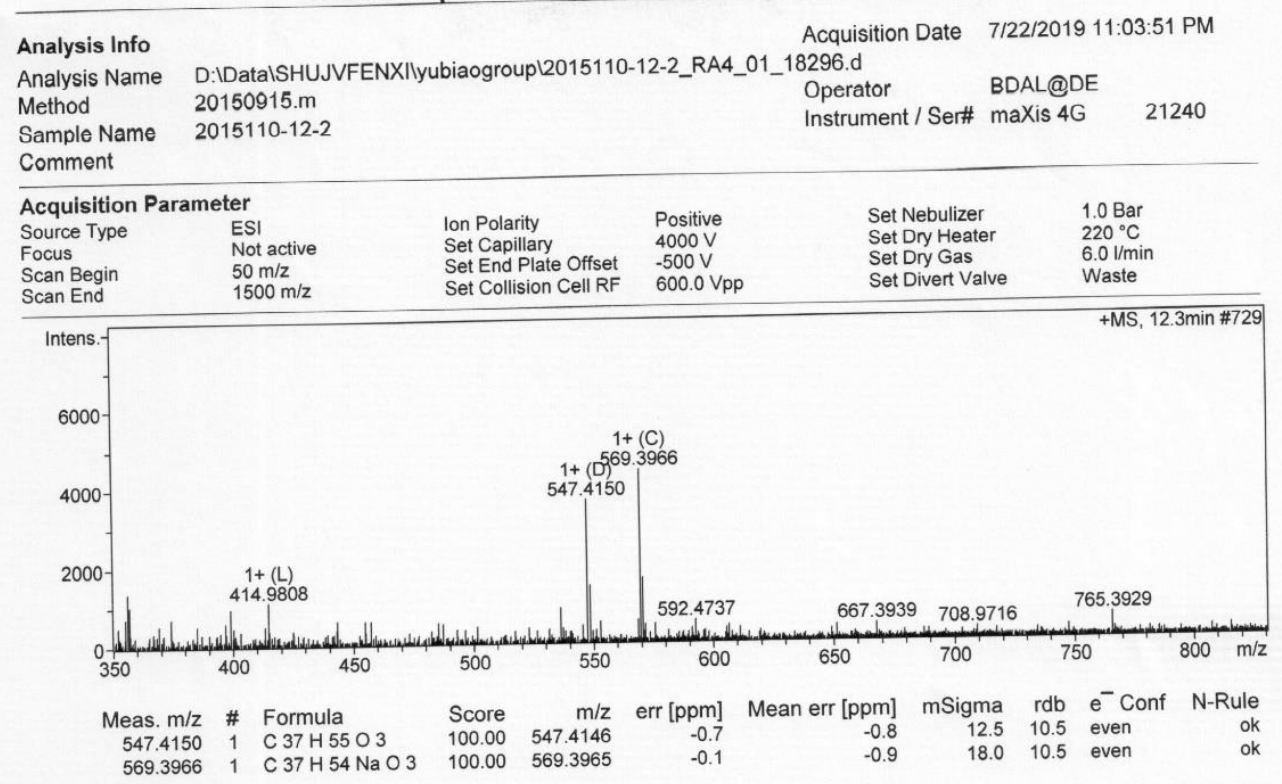

Figure S158. HRMS of compound 44 


\section{Mass Spectrum SmartFormula Report}

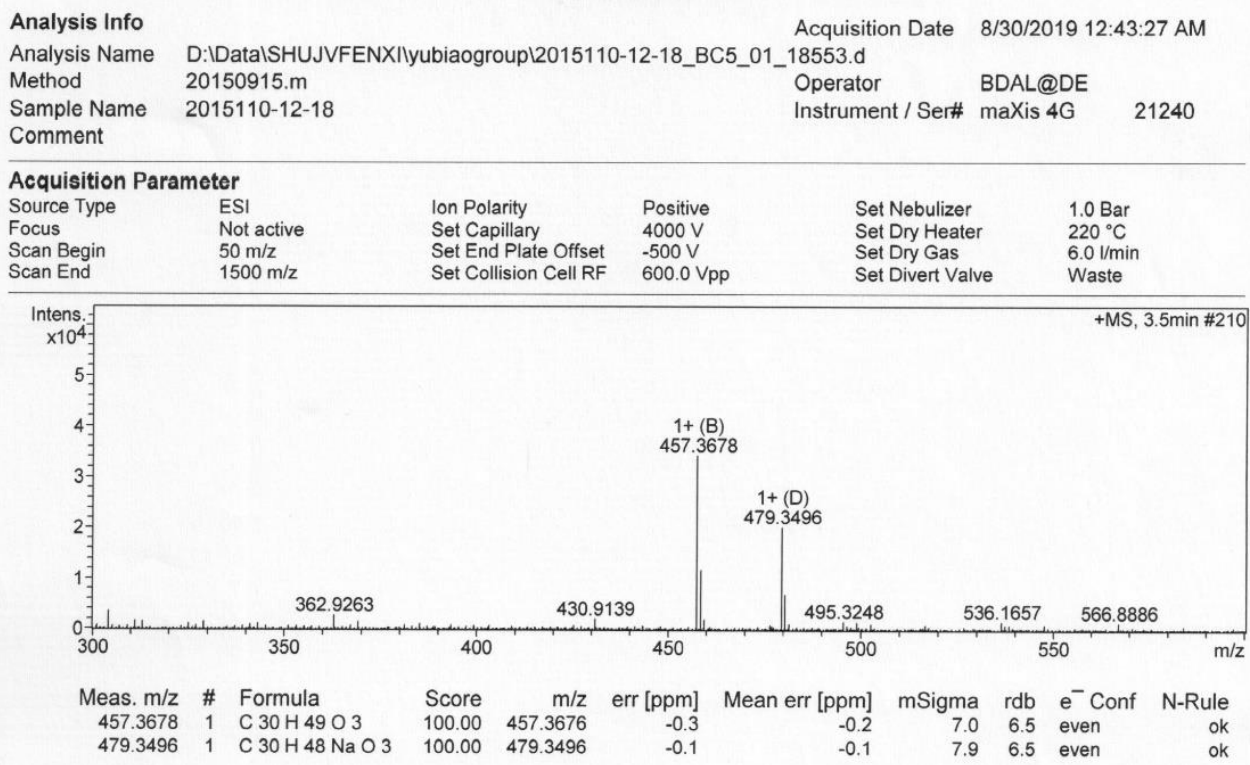

Figure S159. HRMS of compound 45 


\section{Mass Spectrum SmartFormula Report}

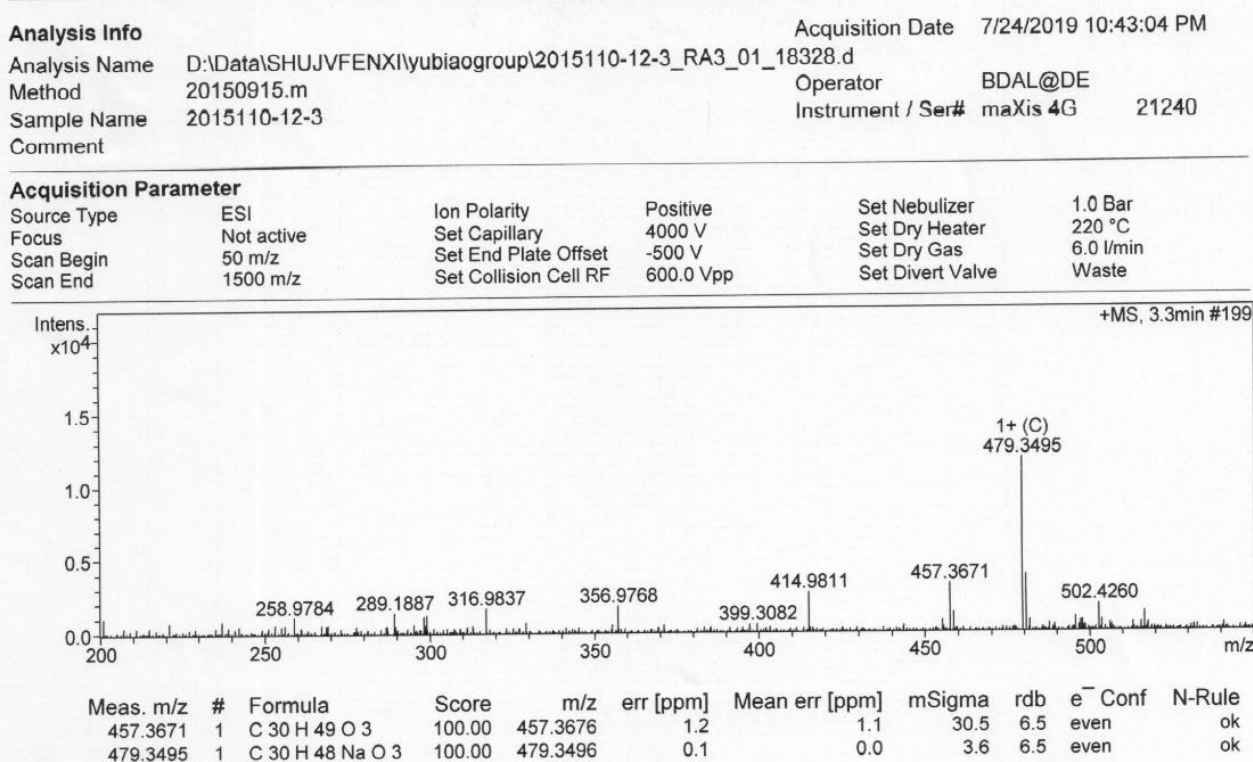

Figure S160. HRMS of compound 46 


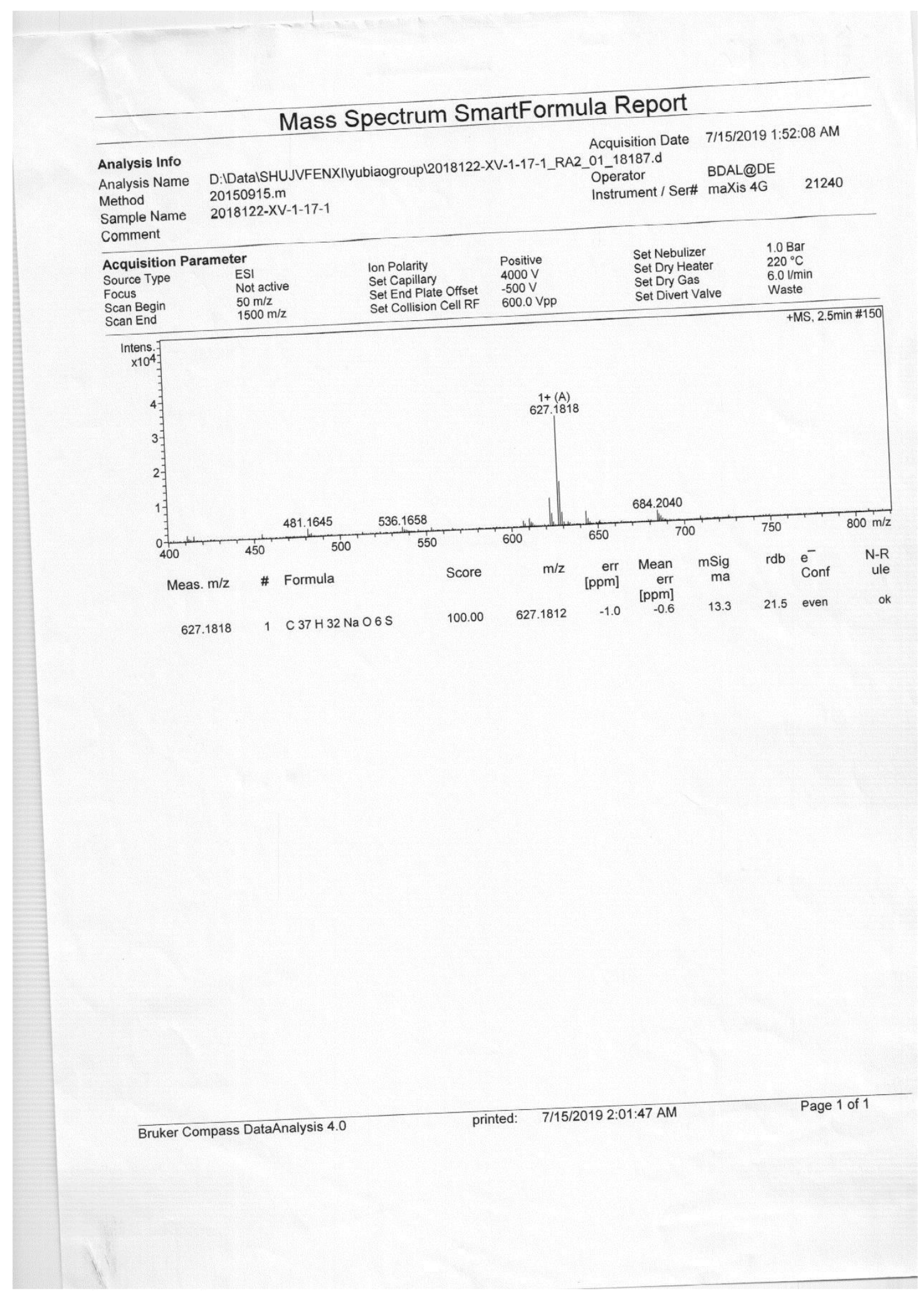

Figure S161. HRMS of $p$-tolyl 2,3-di- $O$-benzoyl-4- $O$-Nap-1-thio- $\beta$-D-xylopyranoside 


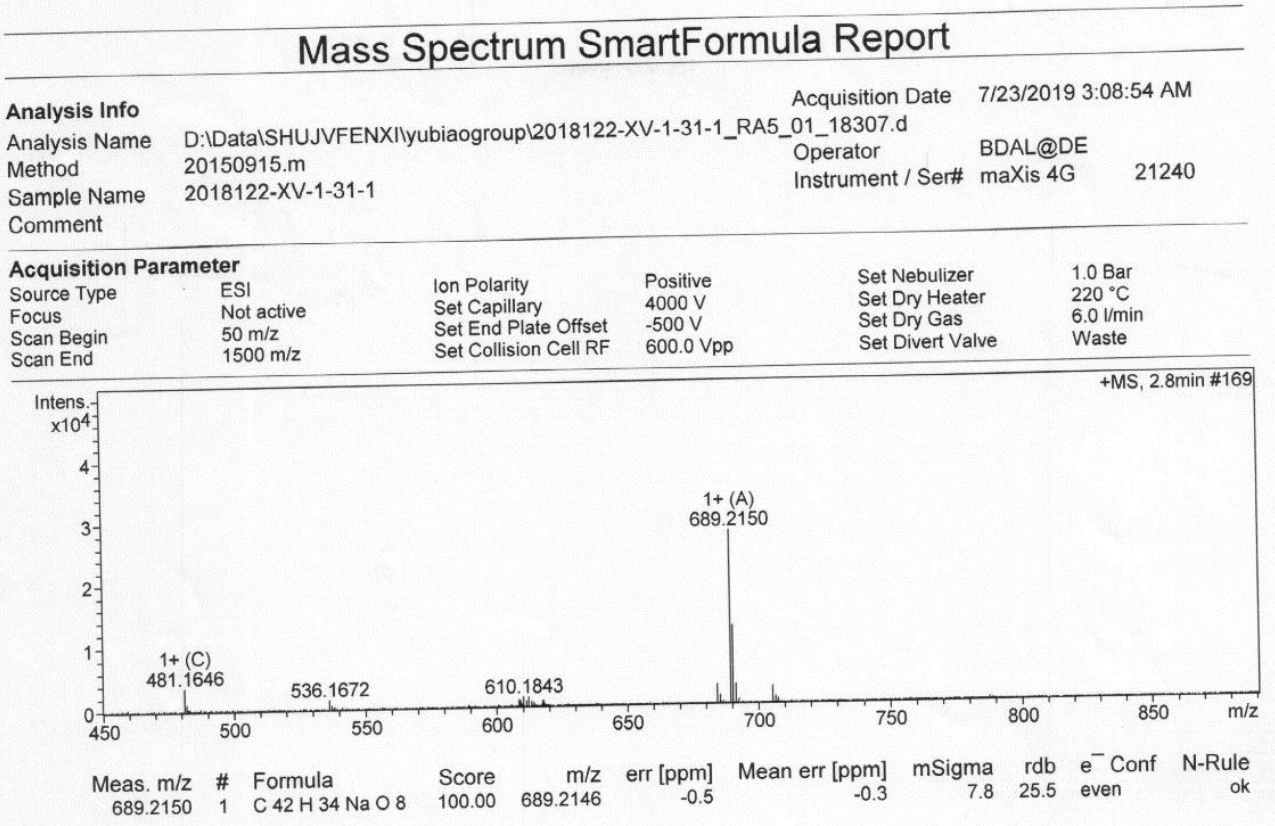

Figure S162. HRMS of compound $47 \alpha$ 


\section{Mass Spectrum SmartFormula Report}

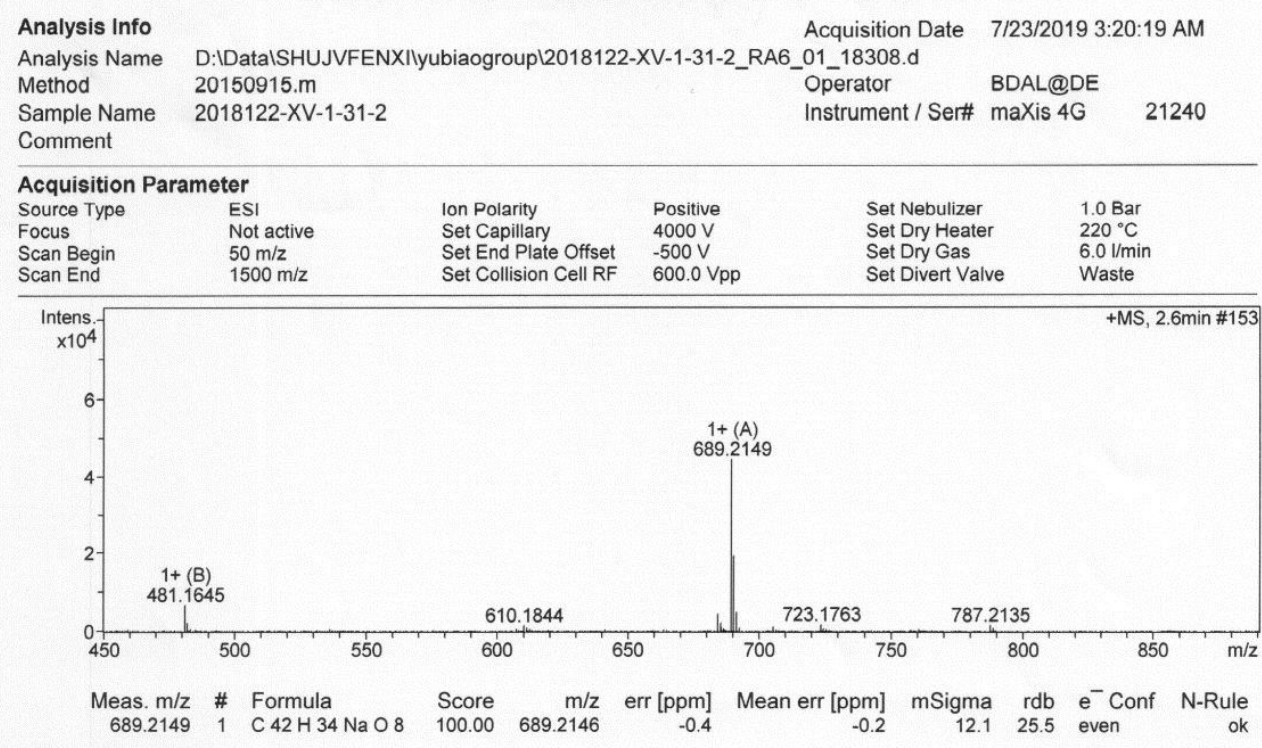

Figure S163. HRMS of compound 47及 
National Center for Organic Mass Spectrometry in Shanghai

Shanghai Institute of Organic Chemistry

Chinese Academic of Sciences

High Resolution MS ESI REPORT

Instrument: Thermo Scientific Q Exactive HF Orbitrap-FTMS

Card Serial Number: E192294

Sample Serial Number: 12-22

Operator: Songw

Date: $2019 / 11 / 02$

Operation Mode: ESI Positive Ion Mode

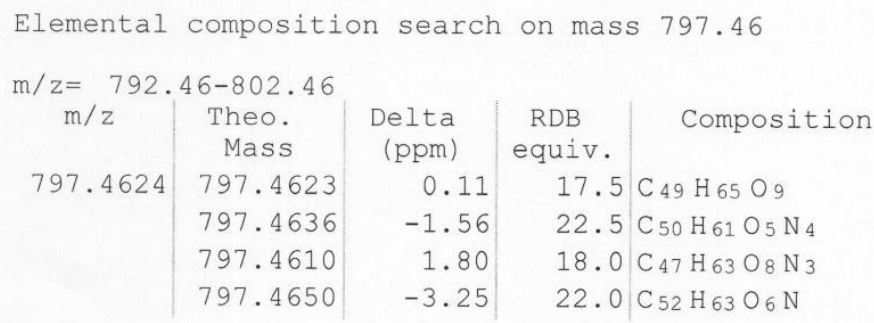

Figure S164. HRMS of compound 48 


\section{Mass Spectrum SmartFormula Report}

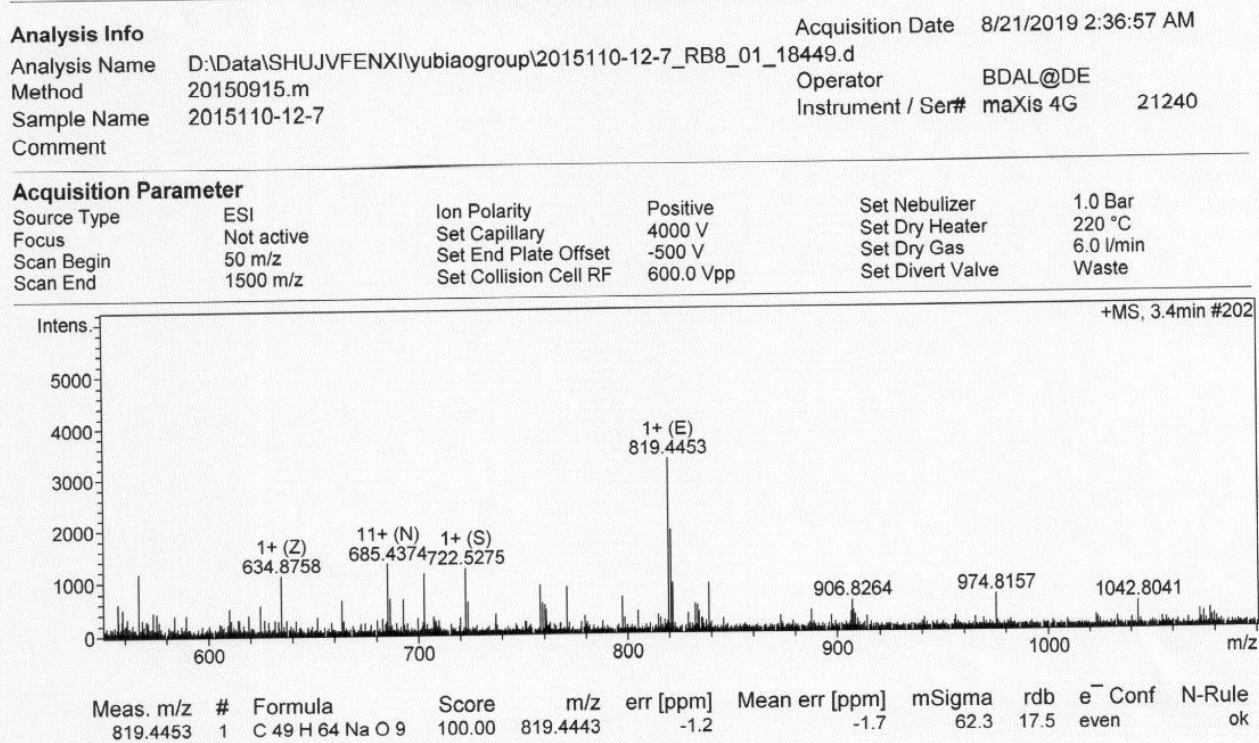

Figure S165. HRMS of compound 49 
National Center for Organic Mass Spectrometry in Shanghai

Shanghai Institute of Organic Chemistry

Chinese Academic of Sciences

High Resolution MS ESI REPORT

Instrument: Thermo Scientific Q Exactive HF Orbitrap-FTMS

Card Serial Number: E192289

Sample Serial Number: 12-20

Operator: Songw Date: 2019/11/02

Operation Mode: ESI Positive Ion Mode

\begin{tabular}{|c|c|c|c|c|}
\hline \multicolumn{5}{|c|}{$\mathrm{m} / \mathrm{z}=880.49-890.49$} \\
\hline $\mathrm{m} / \mathrm{z}$ & $\begin{array}{r}\text { Theo. } \\
\text { Mass }\end{array}$ & $\begin{array}{r}\text { Delta } \\
\text { (ppm) }\end{array}$ & $\begin{array}{l}\text { RDB } \\
\text { equiv. }\end{array}$ & Composition \\
\hline 885.4916 & 885.4912 & 0.42 & 19.5 & $\mathrm{C}_{54} \mathrm{H}_{70} \mathrm{O}_{9} \mathrm{Na}$ \\
\hline & 885.4923 & -0.78 & 23.0 & $\mathrm{C}_{54} \mathrm{H}_{67} \mathrm{O}_{8} \mathrm{~N}_{3}$ \\
\hline & 885.4930 & -1.61 & 15.0 & $\mathrm{C}_{48} \mathrm{H}_{72} \mathrm{O}_{9} \mathrm{~N}_{3} \mathrm{NaSi}$ \\
\hline & 885.4899 & 1.94 & 20.0 & $\mathrm{C}_{52} \mathrm{H}_{68} \mathrm{O}_{8} \mathrm{~N}_{3} \mathrm{Na}$ \\
\hline & 885.4936 & -2.29 & 22.5 & $\mathrm{C}_{56} \mathrm{H}_{69} \mathrm{O}_{9}$ \\
\hline & 885.4954 & -4.32 & 18.0 & 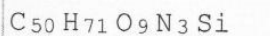 \\
\hline
\end{tabular}

Figure S166. HRMS of compound $\mathbf{5 0}$ 


\section{Mass Spectrum SmartFormula Report}

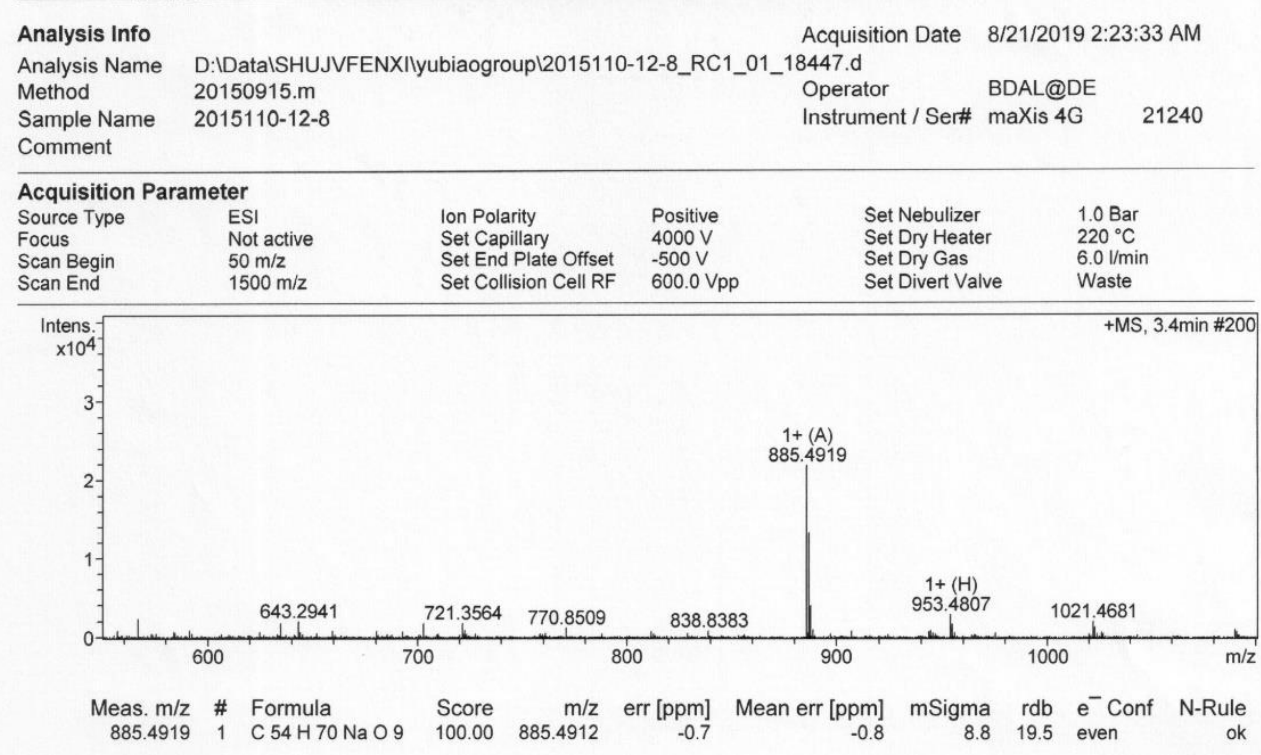

Figure S167. HRMS of compound 51 
National Center for Organic Mass Spectrometry in Shanghai Shanghai Institute of Organic Chemistry

Chinese Academic of Sciences

High Resolution MS ESI REPORT

Instrument: Thermo Scientific Q Exactive HF Orbitrap-FTMS

Card Serial Number: H190722

Sample Serial Number: 12-25

Operator: QHL_ Date: 2019/10/24

Operation Mode: ESI Positive Ion Mode

Elemental composition search on mass 1181.58

$\mathrm{m} / \mathrm{z}=1176.58-1186.58$

\begin{tabular}{|c|r|r|r|l}
$\mathrm{m} / \mathrm{z}$ & Theo. Mass & $\begin{array}{c}\text { Delta } \\
(\mathrm{ppm})\end{array}$ & \multicolumn{1}{|c|}{$\begin{array}{l}\text { RDB } \\
\text { equiv. }\end{array}$} & \multicolumn{1}{|c|}{ Composition } \\
\hline 181.5830 & 1181.5832 & -0.17 & 28.5 & $\mathrm{C}_{70} \mathrm{H}_{85} \mathrm{O}_{16}$ \\
& 1181.5859 & -2.44 & $33.0 \mathrm{C}_{73} \mathrm{H}_{83} \mathrm{O}_{13} \mathrm{~N}$ \\
& 1181.5800 & 2.53 & $42.0 \mathrm{C}_{80} \mathrm{H}_{79} \mathrm{O}_{8} \mathrm{~N}$ \\
& 1181.5773 & 4.80 & 37.5 & $\mathrm{C}_{77} \mathrm{H}_{81} \mathrm{O}_{11}$
\end{tabular}

Figure S168. HRMS of compound 53 


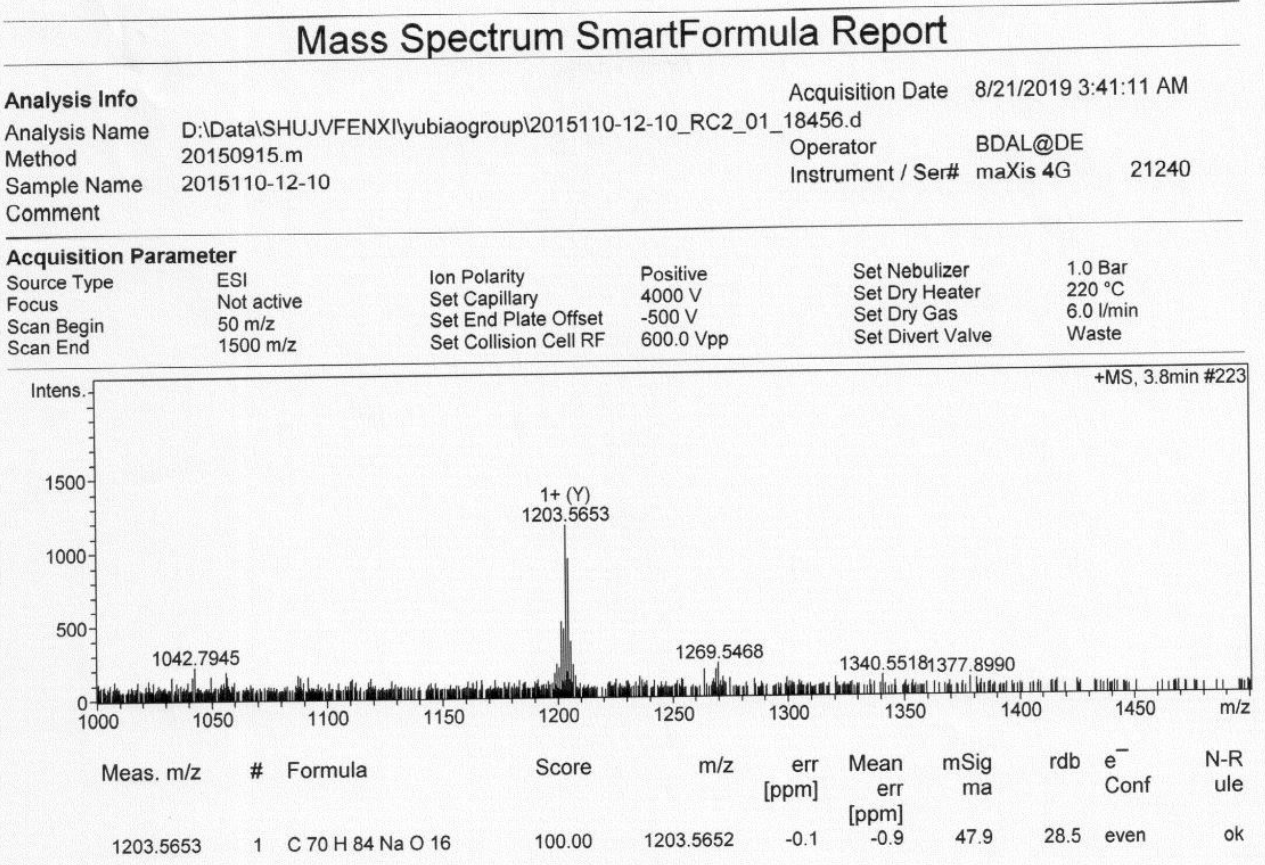

Figure S169. HRMS of compound 54 
National Center for Organic Mass Spectrometry in Shanghai Shanghai Institute of Organic Chemistry

Chinese Academic of Sciences

High Resolution MS ESI REPORT

Instrument: Thermo Scientific Q Exactive HF Orbitrap-FTMS

Card Serial Number: E192291

Sample Serial Number: $12-30$

Operator: Songw

Date: $2019 / 11 / 02$

Operation Mode: ESI Negative Ion Mode

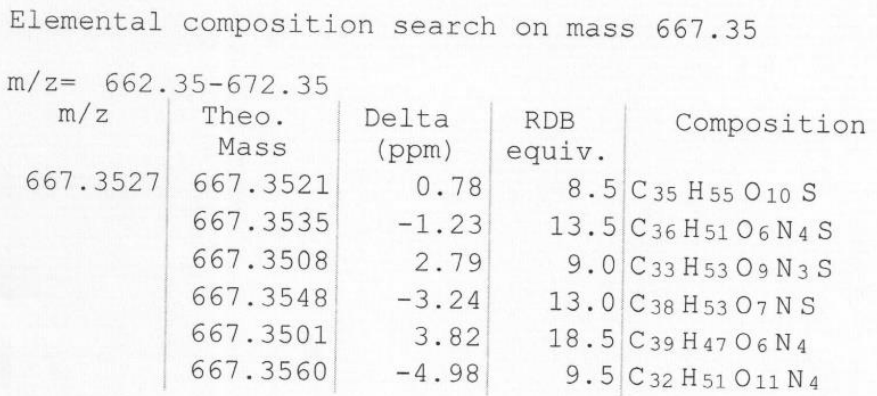

Figure S170. HRMS of compound $\mathbf{5 5}$ 
National Center for Organic Mass Spectrometry in Shanghai Shanghai Institute of Organic Chemistry

Chinese Academic of Sciences

High Resolution MS ESI REPORT

Instrument: Thermo Scientific Q Exactive HF Orbitrap-FTMS

Card Serial Number: H190716

Sample Serial Number: $12-28$

Operator: QHL Date: 2019/10/24

Operation Mode: ESI Positive Ion Mode

Elemental composition search on mass 589.41

$\mathrm{m} / \mathrm{z}=584.41-594.41$

\begin{tabular}{l|c|c|c|c}
$\mathrm{m} / \mathrm{z}$ & Theo. & Delta & RDB & Composition
\end{tabular}

Mass (ppm) equiv.

$\begin{array}{llllll}589.4100 & 589.4099 & 0.15 & 7.5 \mathrm{C}_{35} \mathrm{H}_{57} \mathrm{O}_{7}\end{array}$

$\begin{array}{lll}589.4107 & -1.32 & 6.5 \mathrm{C}_{36} \mathrm{H}_{61} \mathrm{O}_{2} \mathrm{~S}_{2}\end{array}$

$589.4126-4.40 \quad 12.0 \mathrm{C}_{38} \mathrm{H}_{55} \mathrm{O}_{4} \mathrm{~N}$

$\begin{array}{lll}589.4074 & 4.40 & 11.5 \mathrm{C}_{39} \mathrm{H}_{57} \mathrm{O}_{2} \mathrm{~S}\end{array}$

Figure S171. HRMS of compound 56 
National Center for Organic Mass Spectrometry in Shanghai

Shanghai Institute of Organic Chemistry

Chinese Academic of Sciences

High Resolution MS ESI REPORT

Instrument: Thermo Scientific Q Exactive HF Orbitrap-FTMS

Card Serial Number: E192293

Sample Serial Number: 12-16

Operator: Songw Date: 2019/11/02

Operation Mode: ESI Negative Ion Mode

\begin{tabular}{|c|c|c|c|c|}
\hline \multicolumn{5}{|c|}{$\mathrm{m} / \mathrm{z}=662.35-672.35$} \\
\hline $\mathrm{m} / \mathrm{z}$ & $\begin{array}{l}\text { Theo. } \\
\text { Mass }\end{array}$ & $\begin{array}{r}\text { Delta } \\
(\mathrm{ppm})\end{array}$ & $\begin{array}{l}\text { RDB } \\
\text { equiv. }\end{array}$ & Composition \\
\hline \multirow{5}{*}{667.3528} & 667.3521 & 0.96 & 8.5 & $\mathrm{C}_{35} \mathrm{H}_{55} \mathrm{O}_{10} \mathrm{~S}$ \\
\hline & 667.3535 & -1.05 & 13.5 & $\mathrm{C}_{36} \mathrm{H}_{51} \mathrm{O}_{6} \mathrm{~N}_{4} \mathrm{~S}$ \\
\hline & 667.3508 & 2.97 & 9.0 & $\mathrm{C}_{33} \mathrm{H}_{53} \mathrm{O}_{9} \mathrm{~N}_{3} \mathrm{~S}$ \\
\hline & 667.3548 & -3.06 & 13.0 & $\mathrm{C}_{38} \mathrm{H}_{53} \mathrm{O}_{7} \mathrm{~N} \mathrm{~S}$ \\
\hline & 667.3560 & -4.80 & 9.5 & $\mathrm{C}_{32} \mathrm{H}_{51} \mathrm{O}_{11} \mathrm{~N}_{4}$ \\
\hline
\end{tabular}

Figure S172. HRMS of compound 57 


\section{Mass Spectrum SmartFormula Report}

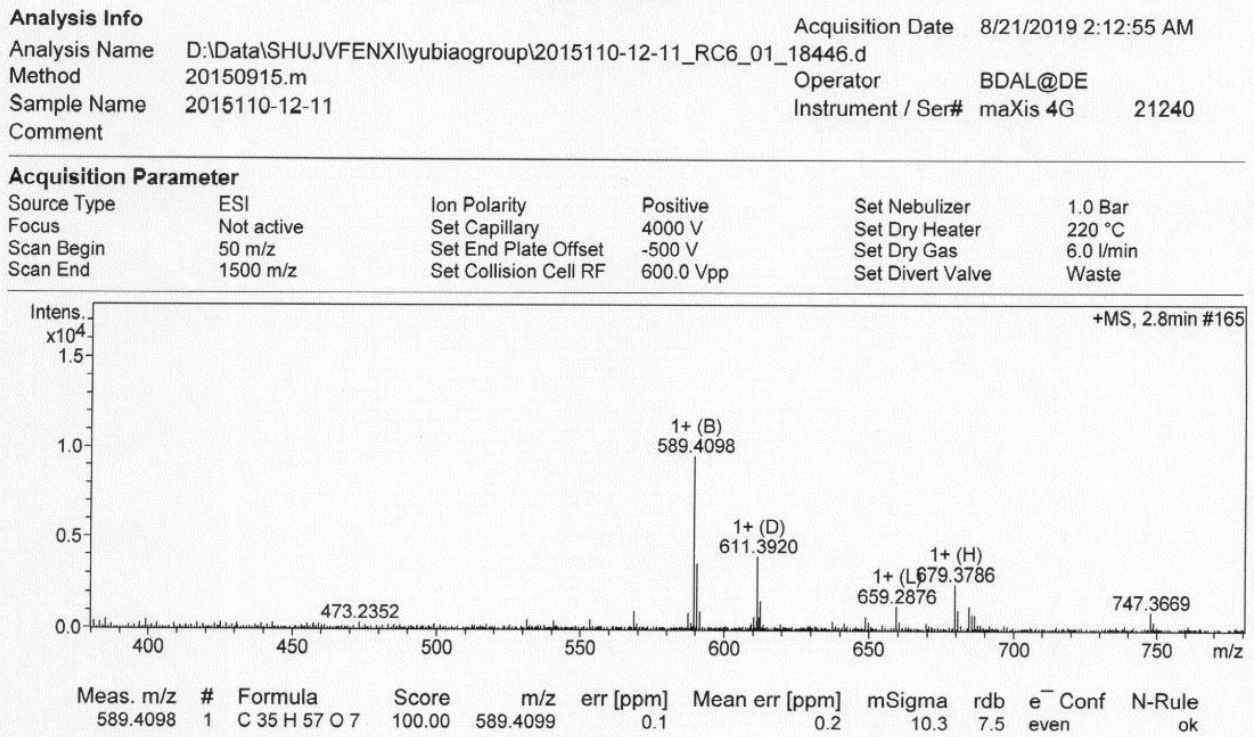

Figure S173. HRMS of compound 58 
National Center for Organic Mass Spectrometry in Shanghai Shanghai Institute of Organic Chemistry

Chinese Academic of Sciences

High Resolution MS ESI REPORT

Instrument: Thermo Scientific Q Exactive HF Orbitrap-FTMS

Card Serial Number: E192290

Sample Serial Number: $12-32$

Operator: Songw

Date: $2019 / 11 / 02$

Operation Mode: ESI Negative Ion Mode

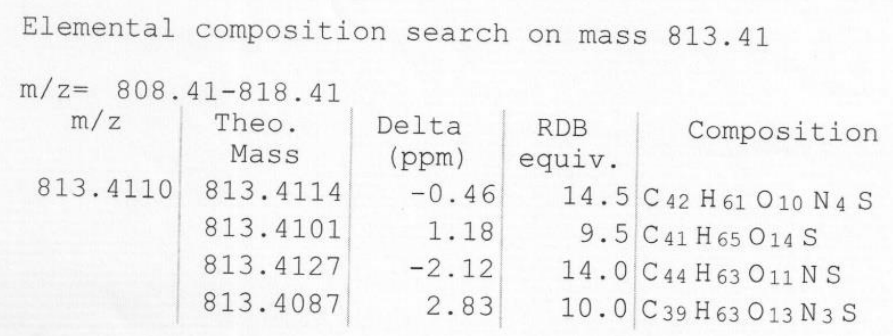

Figure S174. HRMS of compound 59 
National Center for Organic Mass Spectrometry in Shanghai Shanghai Institute of Organic Chemistry

Chinese Academic of Sciences

High Resolution MS ESI REPORT

Instrument: Thermo Scientific Q Exactive HF Orbitrap-FTMS

Card Serial Number: H190715

Sample Serial Number: 12-27

Operator: QHL_ Date: 2019/10/24

Operation Mode: ESI Positive Ion Mode

Elemental composition search on mass 735.47

$\mathrm{m} / \mathrm{z}=730.47-740.47$

\begin{tabular}{l|l|l|l|l}
$\mathrm{m} / \mathrm{z}$ & Theo. & Delta & $\mathrm{RDB}$ & Composition
\end{tabular}

\begin{tabular}{l|l|l} 
& Mass (ppm) equiv.
\end{tabular}

\begin{tabular}{l|r|r|r|r}
735.4674 & 735.4678 & -0.60 & $8.5 \mathrm{C}_{41} \mathrm{H}_{67} \mathrm{O}_{11}$
\end{tabular}

\begin{tabular}{l|l|l|l}
735.4653 & 2.81 & $12.5 \mathrm{C}_{45} \mathrm{H}_{67} \mathrm{O}_{6} \mathrm{~S}$
\end{tabular}

\begin{tabular}{ll|l|l}
735.4705 & -4.24 & $13.0 \mathrm{C}_{44} \mathrm{H}_{65} \mathrm{O}_{8} \mathrm{~N}$
\end{tabular}

Figure S175. HRMS of compound 60 
National Center for Organic Mass Spectrometry in Shanghai Shanghai Institute of Organic Chemistry

Chinese Academic of Sciences

High Resolution MS ESI REPORT

Instrument: Thermo Scientific Q Exactive HF Orbitrap-FTMS

Card Serial Number: E192292

Sample Serial Number: $12-13$

Operator: Songw Date: 2019/11/02

Operation Mode: ESI Negative Ion Mode

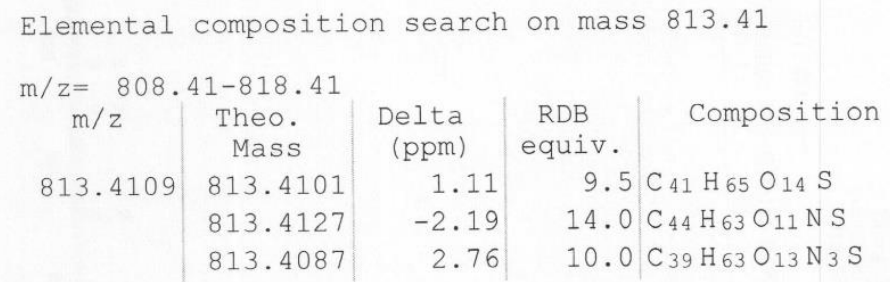

Figure S176. HRMS of compound 61 


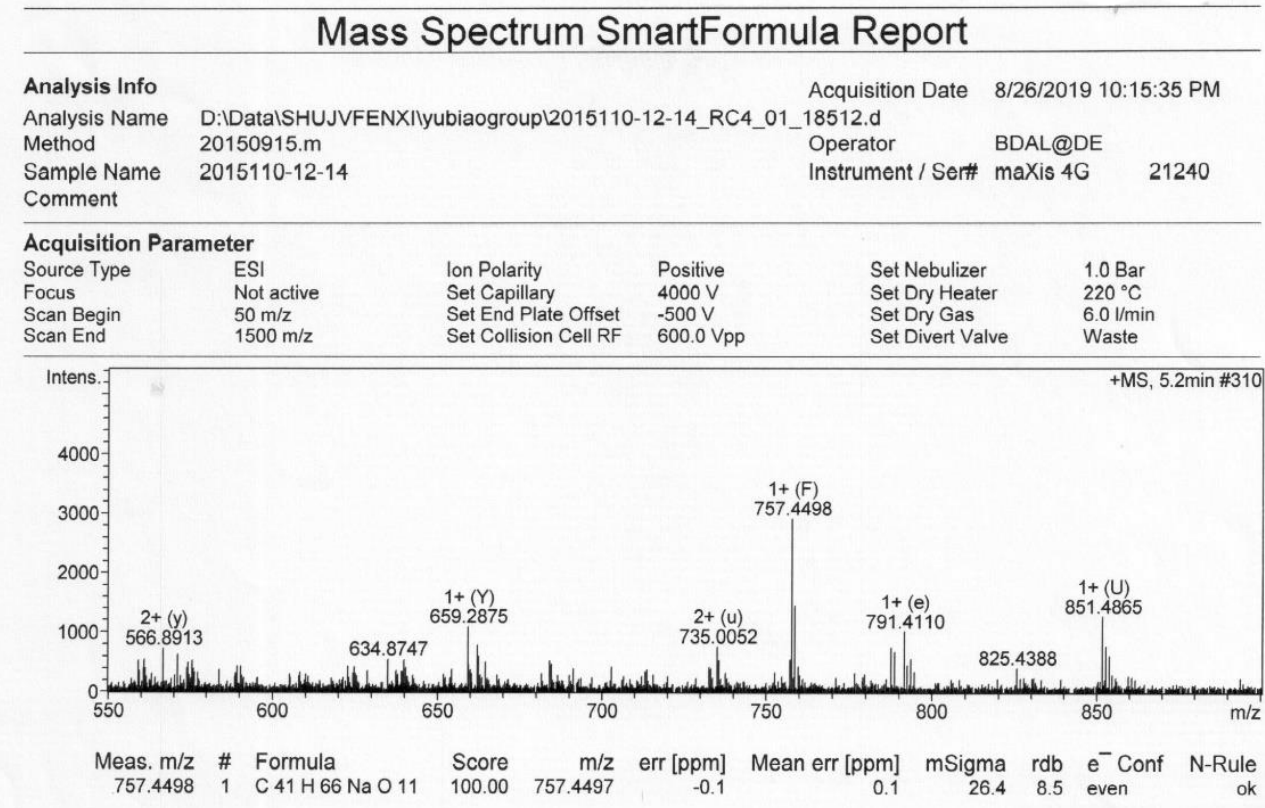

Figure S177. HRMS of compound 62 